\title{
Building Energy Simulation Test for Existing Homes (BESTEST-EX)
}

Technical Report NREL/TP-550-47427 August 2010

\section{Phase 1 Test Procedure: Building Thermal Fabric Cases}

Ron Judkoff, Ben Polly, and Marcus Bianchi National Renewable Energy Laboratory

Joel Neymark

J. Neymark \& Associates

Link to Accompanying Zipped Data Files (2.9 MB) 


\section{Building Energy Simulation Test for Existing Homes (BESTEST-EX)}

Phase 1 Test Procedure: Building Thermal Fabric Cases

Ron Judkoff, Ben Polly, and Marcus Bianchi National Renewable Energy Laboratory

Joel Neymark

J. Neymark \& Associates

Prepared under Task No. ARRB.1000

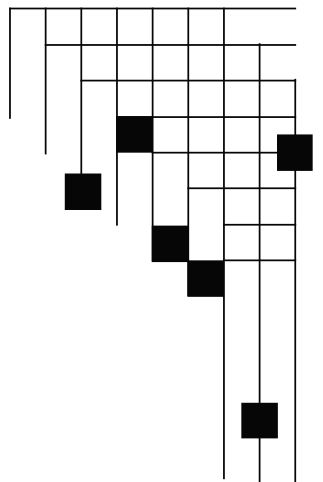




\section{NOTICE}

This report was prepared as an account of work sponsored by an agency of the United States government. Neither the United States government nor any agency thereof, nor any of their employees, nor any of their contributors, makes any warranty, express or implied, or assumes any legal liability or responsibility for the accuracy, completeness, or usefulness of any information, apparatus, product, or process disclosed, or represents that its use would not infringe privately owned rights. Reference herein to any specific commercial product, process, or service by trade name, trademark, manufacturer, or otherwise does not necessarily constitute or imply its endorsement, recommendation, or favoring by the United States government or any agency thereof. The views and opinions of authors expressed herein do not necessarily state or reflect those of the United States government or any agency thereof.

Available electronically at http://www.osti.gov/bridge

Available for a processing fee to U.S. Department of Energy and its contractors, in paper, from:

U.S. Department of Energy

Office of Scientific and Technical Information

P.O. Box 62

Oak Ridge, TN 37831-0062

phone: 865.576 .8401

fax: 865.576 .5728

email: mailto:reports@adonis.osti.gov

Available for sale to the public, in paper, from:

U.S. Department of Commerce

National Technical Information Service

5285 Port Royal Road

Springfield, VA 22161

phone: 800.553.6847

fax: 703.605.6900

email: orders@ntis.fedworld.gov

online ordering: http://www.ntis.gov/ordering.htm 


\section{Acknowledgments}

For feedback and comments on the test procedure provided by BESTEST-EX Working Group participants during preliminary simulation trials, we wish to thank the following companies and individuals that ran simulations and responded to questionnaires: Apogee Interactive Inc.: Joel Gilbert, John Laun, Eric Shewbridge, Lei Wang; Architectural Energy Corporation: Brian Christensen, Rob Salcido; Conservation Services Group: Bruce Harley, Mick Rookwood, David Weitz, Michael Blasnik (consultant); Florida Solar Energy Center: Philip Fairey; ICF International: Brian Dean, Mike L'Ecuyer; Oak Ridge National Laboratory: Mark Ternes; Performance Systems Development LLC: Chris Balbach, Ethan MacCormick, John McCartney, Greg Thomas.

Paul Norton of the National Renewable Energy Laboratory (NREL) provided initial organizational and management support during project startup. Ren Anderson of NREL also provided managerial support. Nick Long of NREL provided assistance with automating reference simulations.

We appreciate the support and guidance of Ed Pollock, Terry Logee, and Lew Pratsch, of the U.S. Department of Energy. 


\section{Nomenclature}

A

Abs

Abs In

Abs Out

$\mathrm{ACH}$

$\mathrm{ACH}_{50}$

AIR

$\boldsymbol{\alpha}_{\text {ext }}$

ASHRAE

BA

BESTEST

BoC

"C.C"

C3

C4

C5

C6

C7

cfm

CFM50 or

$\mathrm{CFM}_{50}$

CO or Col

Springs

Coef or Coeff

COG

COP

$\mathrm{COP}_{\text {clg }}$

Corr.

Cp

D

DHW

Dir. nor.

DOE

DOE2.1E

$\mathbf{E}_{\text {htg }}$

E+

EIA

ELA

EOG

Ext.

F

$\mathbf{h}_{\mathbf{c}}$

HEATCAP

Hemis

HERS

$\mathbf{h}_{\mathbf{i}}$

$\mathbf{h}_{\mathbf{0}}$

$\mathbf{h}_{\text {s }}$ area

absorptance

absorptance of inner pane

absorptance of outer pane

air changes per hour

air changes per hour at a pressure difference of 50 Pascals

approximate input range

exterior solar absorptance

American Society of Heating, Refrigerating and Air-Conditioning Engineers

Building America

Building Energy Simulation Test

benefit of calibration

calibrated energy savings test cases

fully random explicit input selection, near-nominal space heating/cooling

consumption

fully random explicit input selection, high space heating/cooling consumption

fully random explicit input selection, low space heating/cooling consumption

fully random explicit input selection, mid-high space heating/cooling consumption

fully random explicit input selection, mid-low space heating/cooling consumption

cubic feet per minute

infiltration flow rate (in $\mathrm{cfm}$ ) at a pressure difference of 50 Pascals

Colorado Springs, Colorado

coefficient

center of glass

coefficient of performance

effective space cooling system coefficient of performance

correction

specific heat, $\mathrm{Btu} /\left(\mathrm{lb} \cdot{ }^{\circ} \mathrm{F}\right)[\mathrm{J} /(\mathrm{kg} \cdot \mathrm{K})]$

door $3^{\prime} \times 6^{\prime} 8^{\prime \prime}$

domestic hot water

direct normal

U.S. Department of Energy

DOE-2.1E, Version JJHirsch PC 2.1 En136

effective space heating system efficiency

EnergyPlus, Version 3.1

Energy Information Administration

effective leakage area

edge of glass

exterior

${ }^{\circ} \mathrm{F}$ (used in units designators)

convective surface heat transfer coefficient

heat capacity

hemispherical

Home Energy Rating System

infrared radiative surface heat transfer coefficient

combined convective and infrared radiative exterior surface heat transfer coefficient

combined convective and infrared radiative interior surface heat transfer coefficient 


\begin{tabular}{|c|c|}
\hline HVAC & heating, ventilating, and air-conditioning \\
\hline Ins & insulation \\
\hline Int & interior \\
\hline ISO & International Standards Organization \\
\hline I-P & inch-pound \\
\hline $\mathbf{k}$ & thermal conductivity, $\mathrm{Btu} /\left(\mathrm{h} \cdot \mathrm{ft} \cdot{ }^{\circ} \mathrm{F}\right)[\mathrm{W} /(\mathrm{m} \cdot \mathrm{K})]$ \\
\hline LBL & Lawrence Berkeley Laboratory, or Lawrence Berkeley National Laboratory \\
\hline Low-e & low emissivity \\
\hline $\mathbf{L V}$ & Las Vegas, Nevada \\
\hline Max & maximum, also approximate input range maximum \\
\hline Min & minimum, also approximate input range minimum \\
\hline NAHB & National Association of Home Builders \\
\hline NCDC & National Climatic Data Center \\
\hline NFRC & National Fenestration Rating Council \\
\hline Nom & nominal \\
\hline NREL & National Renewable Energy Laboratory \\
\hline NSRDB & National Solar Radiation Data Base \\
\hline N/A & not applicable \\
\hline o.c. or O.C. & on centers \\
\hline “-P” & building physics test cases \\
\hline $\mathbf{R}$ & unit thermal resistance, $\mathrm{h} \cdot \mathrm{ft}^{2} \cdot{ }^{\circ} \mathrm{F} / \mathrm{Btu}\left[\mathrm{m}^{2} \cdot \mathrm{K} / \mathrm{W}\right]$ \\
\hline REF & $\begin{array}{l}\text { average of reference program energy savings predictions using randomly selected } \\
\text { explicit inputs (million Btu or } \mathrm{kWh} \text { ) }\end{array}$ \\
\hline Refl. & reflectance \\
\hline Refl,f & reflectance for radiation incident from the front (from the exterior surface) \\
\hline Refl,b & reflectance for radiation incident from the back (from the interior surface) \\
\hline SC & shading coefficient \\
\hline SHGC & solar heat gain coefficient \\
\hline sol_abs & solar absorptance \\
\hline SUNĒELL & SUNREL, Version 1.14 \\
\hline Surf & surface \\
\hline TEMP & temperature \\
\hline $\mathbf{T}_{\mathrm{clg}}$ & zone air temperature for cooling \\
\hline $\mathbf{T}_{\text {htg }}$ & zone air temperature for heating \\
\hline TMY & Typical Meteorological Year \\
\hline TMY2 or TM2 & Typical Meteorological Year 2 \\
\hline Trans. & transmittance \\
\hline $\mathbf{U}$ & $\begin{array}{l}\text { unit thermal conductance or overall heat transfer coefficient, } \mathrm{Btu} /\left(\mathrm{h} \cdot \mathrm{ft}^{2} \cdot{ }^{\circ} \mathrm{F}\right) \\
{\left[\mathrm{W} /\left(\mathrm{m}^{2} \cdot \mathrm{K}\right)\right]}\end{array}$ \\
\hline UA & thermal conductance \\
\hline $\mathbf{U} \mathbf{A}_{\text {inf }}$ or $\mathbf{U} \mathbf{A}_{\text {infl }}$ & equivalent thermal conductance due to infiltration \\
\hline UV & ultraviolet \\
\hline Val & value \\
\hline $\mathbf{W}$ & Window, $3^{\prime} \times 5^{\prime}$ \\
\hline WBAN & Weather Bureau Army Navy \\
\hline WG CAL & average working group calibrated energy savings prediction (million Btu or $\mathrm{kWh}$ ) \\
\hline WG UNCAL & average working group uncalibrated energy savings prediction (million Btu or $\mathrm{kWh}$ ) \\
\hline
\end{tabular}




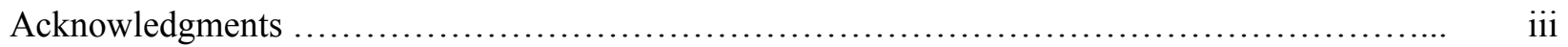



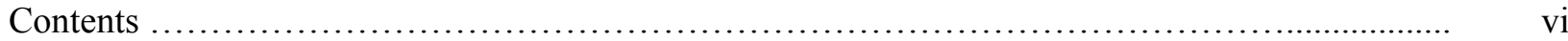

Accompanying Files (Electronic Media Contents) ..................................... viii

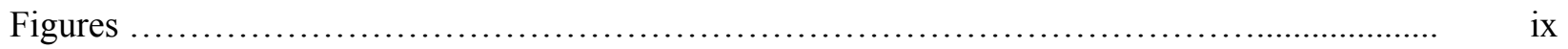

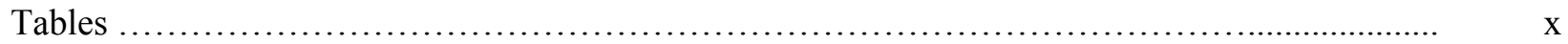

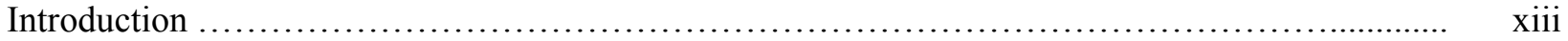

Overview of the BESTEST-EX Phase 1 Test Suite ....................................... xiii

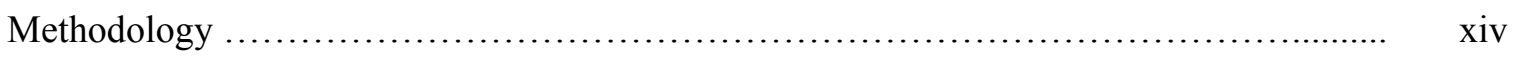

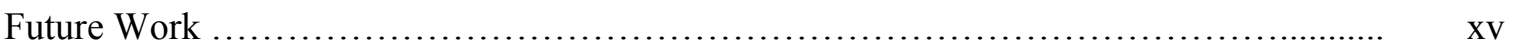

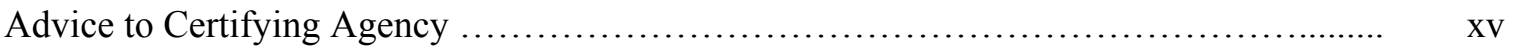

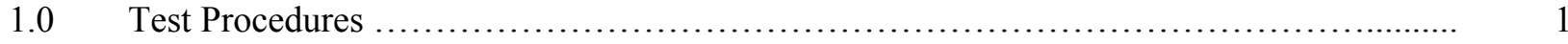

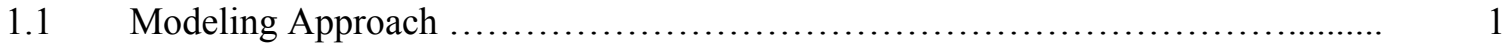

1.1.1 Time Convention ..................................................... 1

1.1.2 Geometry Convention ......................................... 1

1.1.3 Nonapplicable Inputs ....................................... 1

1.1.4 Consistent Modeling Methods ...................................... 1

1.1.5 Equivalent Modeling Methods ..................................... 1

1.1.6 Simulation Initialization and Preconditioning ....................... 1

1.1.7 Simulation Duration ................................................. 1

1.1.8 Programs With Different Operational Modes Depending on Utility Data Availability .................................................... 1

1.1.9 Order of Testing ................................................... 2

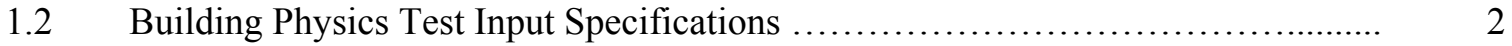

1.2.1 The Pre-Retrofit Base-Case Building (Case L200EX-P) .................... 3

1.2.2 Building Physics Retrofit Test Cases .................................. 29

1.3 Calibrated Energy Savings Tests Input Specifications ............................. 46

1.3.1 Pre-Retrofit Base-Case Building (Case L200EX-C) ....................... 46

1.3.2 Calibrated Energy Savings Retrofit Test Cases ........................ 58

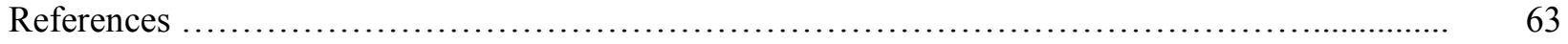




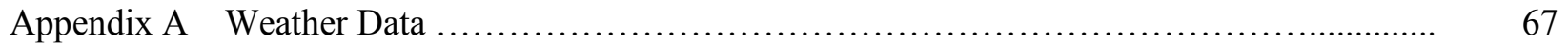

Appendix B Selection of Internal Loads ............................................... 77

Appendix C Combined Surface Heat Transfer Coefficients …............................. 81

Appendix D Infiltration Modeling ................................................ 93

Appendix E Window Modeling with WINDOW 5 . ..................................... 96

Appendix F Random Selection of Explicit Inputs for Case L200EX-C Reference Simulation

Results ............................................................... 100

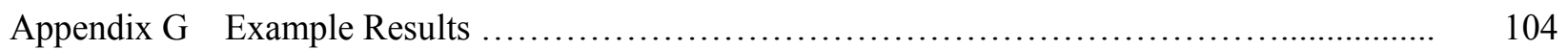

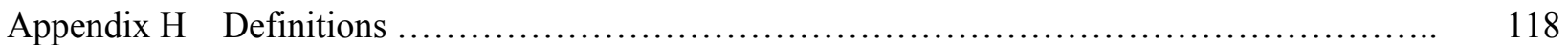

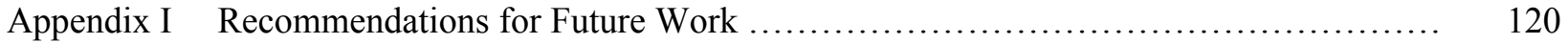




\section{Accompanying Files (Electronic Media Contents)}

The following files provided within BESTEST-EX-Phase-1-Proc-AccompanyingFiles.zip apply as they are called out in the test procedure:

- README-BESTEST-EX-Files.doc: Electronic media contents.

- Colorad.TM2: TMY2 weather data for Colorado Springs, Colorado, as described in Appendix A.

- Lasvega.TM2: TMY2 weather data for Las Vegas, Nevada, as described in Appendix A.

- BESTEST-EX-Phase-1-Output.xls: Spreadsheet standard output report for entering results.

- B-EX-Phase-1-Ref-P-Results.xls: Spreadsheet that contains reference simulation results presented in Appendix G, Section G.1. Use BESTEST-EX-Phase-1-Output.xls to enter simulation results for the program being tested.

The following reference simulation input files are provided for informative use.

The subfolder B-EX-Ref-Simulation-Physics-Input-Files contains reference simulation input files developed by NREL for the building physics (“-P”) test cases of BESTEST-EX. The input files are organized in lower-tier subfolders as follows:

\section{Subfolder}

DOE-2

EnergyPlus

ISUNREL

B-EX-Ref-Simulation-Weather-Files

\section{$\underline{\text { Reference simulation program }}$}

DOE-2.1E Version JJHirsch PC 2.1En136

EnergyPlus Version 3.1

SUNREL Version 1.14

All programs

Reference simulation input files are described further within README-BESTEST-EX-Files.doc. 


\section{Figures}

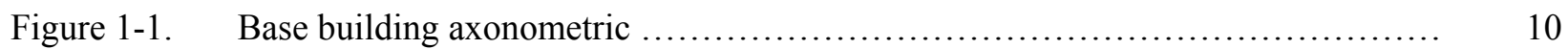

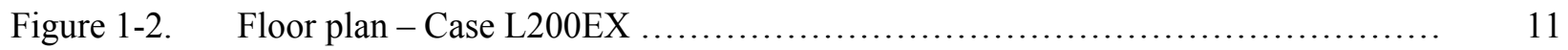

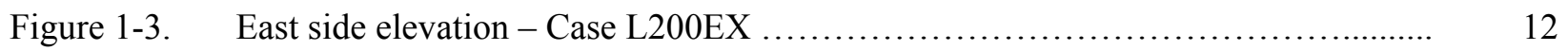

Figure 1-4 Exterior wall plan section - Case L200EX .................................. 13

Figure 1-5. Raised floor exposed to air section - Case L200EX ........................ 13

Figure 1-6. Ceiling/attic/roof section - Case L200EX ............................. 14

Figure 1-7. Interior wall plan section - Case L200EX .................................. 15

Figure 1-8. Window detail, vertical slider (NFRC AA) with 23/4"-wide frame-Case L200EX . 16

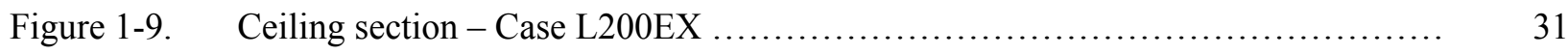

Figure 1-10. Exterior wall plan section - Case L225EX ............................... 34

Figure 1-11. South overhang - Case L270EX-P .................................... 44

Figure 1-12. Overhang for east and west windows - Case L270EX-P ..................... 45

Figure A-1. Sample file header and data in the TMY2 format for January 1 . .................. 70

Figure B-1. Normalized hourly profiles for internal loads due to occupants, gas, and

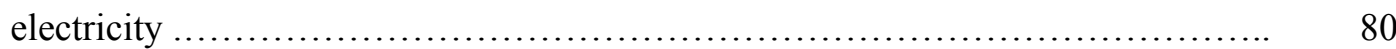

Figure C1-1. Effect of window interior convective surface coefficient on average reference results for gas use and savings in cases with space heating $\ldots \ldots \ldots \ldots \ldots \ldots \ldots \ldots \ldots . \quad 85$

Figure C1-2. Effect of window interior convective surface coefficient on average reference results for electricity use and savings in cases with space cooling ..................

Figure F-1. Triangular probability distribution assumed for random generation of explicit

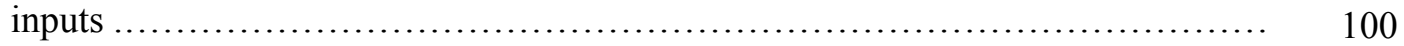

Figure G-1. Building physics heating tests: Reference simulation results.................... 105

Figure G-2. Building physics cooling tests: Reference simulation results................... 105

Figure G-3. Field trial results for fully random, near nominal heating scenario $(\mathrm{C} 3 \mathrm{H}) \ldots \ldots \ldots . .109$

Figure G-4. Field trial results for fully random, high heating scenario $(\mathrm{C} 4 \mathrm{H}) \ldots \ldots \ldots \ldots \ldots \ldots \ldots$

Figure G-5. Field trial results for fully random, low heating scenario $(\mathrm{C} 5 \mathrm{H}) \ldots \ldots \ldots \ldots \ldots \ldots \ldots$

Figure G-6. Field trial results for fully random, mid-high heating scenario $(\mathrm{C} 6 \mathrm{H}) \ldots \ldots \ldots \ldots \ldots . \quad 111$

Figure G-7. Field trial results for fully random, mid-low heating scenario $(\mathrm{C} 7 \mathrm{H}) \ldots \ldots \ldots \ldots \ldots . \quad 111$

Figure G-8. Field trial results for fully random, near nominal cooling scenario (C3C) ........ 112

Figure G-9. Field trial results for fully random, high cooling scenario (C4C) ................ 112

Figure G-10. Field trial results for fully random, low cooling scenario (C5C) ................. 113

Figure G-11. Field trial results for fully random, mid-high cooling scenario (C6C) ............ 113

Figure G-12. Field trial results for fully random, mid-low cooling scenario (C7C) .............. 114 


\section{Tables}

Table 1. BESTEST-EX Case Summary …............................................... xvii

Table 1-1. Building Thermal Summary - Case L200EX ................................ 17

Table 1-2. Other Building Details - Case L200EX ..................................... 18

Table 1-3. Component Surface Areas and Solar Fractions - Case L200EX ...................... 19

Table 1-4. $\quad$ Material Descriptions Exterior Wall, Door, and Window - Case L200EX ............ 20

Table 1-5. Material Descriptions, Raised Floor Exposed to Air - Case L200EX ................ 21

Table 1-6a. Material Descriptions, Ceiling, Attic, and Roof - Case L200EX ...................... 22

Table 1-6b. Material Descriptions, Ceiling/Attic/Roof, Attic as Material Layer Case L200EX

Table 1-7. Material Descriptions, Interior Wall - Case L200EX

Table 1-8a. Conditioned Zone Equivalent Inputs for Weather-Driven Infiltration Models -

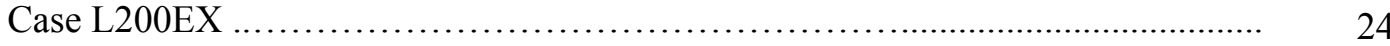

Table 1-8b. Equivalent Seasonal Constant Infiltration ACH and CFM - Case L200EX ........... 25

Table 1-9a. $\quad$ Daily Sensible Internal Loads - Case L200EX …............................. 25

Table 1-9b. Normalized Hourly Profiles for Sensible Internal Loads - Case L200EX ............. 26

Table 1-10. Window Summary (Single-Pane Aluminum Frame With Thermal Breaks) Case L200EX

Table 1-11. Glazing Summary, Single-Pane Center of Glass Values - Case L200EX .............. 28

Table 1-12. Optical Properties as a Function of Incidence Angle for Single-Pane Glazing Case L200EX

Table 1-13. Conditioned Zone Equivalent Inputs for Weather-Driven Infiltration Models Case L210EX-P ................................................................ $\quad 30$

Table 1-14. Equivalent Seasonal Constant Infiltration ACH and CFM - Case L210EX-P ...... 30

Table 1-15. Building Thermal Summary - Case L220EX .................................. 32

Table 1-16a. Material Descriptions, Ceiling - Case L220EX ........................................ 32

Table 1-16b. Material Descriptions for Attic as Material Layer - Case L220EX ................ 33

Table 1-17. Building Thermal Summary - Case L225EX .................................. 35

Table 1-18. Material Descriptions, Exterior Wall - Case L225EX ............................... 35

Table 1-19. Building Thermal Summary - Case L250EX .............................. 38

Table 1-20. Window Summary (Double-Pane, Low-E, Argon Fill, Wood Frame, Insulated Spacer) - Case L250EX ................................................. 39

Table 1-21. Low-E Glazing With Argon Gas Fill Glazing Summary (Center of Glass Values) - Case L250EX ............................................................... 40

Table 1-22. Optical Properties as a Function of Incidence Angle for Low-Emissivity DoublePane Glazing - Case L250EX ....................................... 41

Table 1-23. Component Solar Fractions - Case L250EX …....................................... 42

Table 1-24a. $\quad$ Case L200EX-C1H Reference Utility Energy Use Data.................................. 49 
Table 1-24b. Case L200EX-C2H Reference Utility Energy Use Data................................. 49

Table 1-24c. Case L200EX-C3H Reference Utility Energy Use Data................................. 50

Table 1-24d. Case L200EX-C4H Reference Utility Energy Use Data.................................... 50

Table 1-24e. Case L200EX-C5H Reference Utility Energy Use Data................................. 51

Table 1-24f. Case L200EX-C6H Reference Utility Energy Use Data.................................. 51

Table 1-24g. Case L200EX-C7H Reference Utility Energy Use Data................................... 52

Table 1-25a. Case L200EX-C1C Reference Utility Energy Use Data................................. 52

Table 1-25b. Case L200EX-C2C Reference Utility Energy Use Data................................ 53

Table 1-25c. Case L200EX-C3C Reference Utility Energy Use Data.................................. 53

Table 1-25d. Case L200EX-C4C Reference Utility Energy Use Data................................... 54

Table 1-25e. Case L200EX-C5C Reference Utility Energy Use Data................................. 54

Table 1-25f. Case L200EX-C6C Reference Utility Energy Use Data................................. 55

Table 1-25g. Case L200EX-C7C Reference Utility Energy Use Data................................. 55

Table 1-26. Conditioned Zone Equivalent Input Decrease for Infiltration Models -

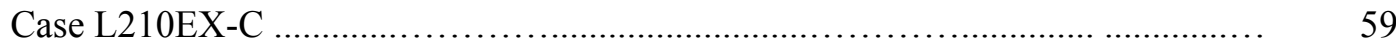

Table A-1. Site and Weather Data Summary for Colorad.TM2 Weather, Colorado Springs,

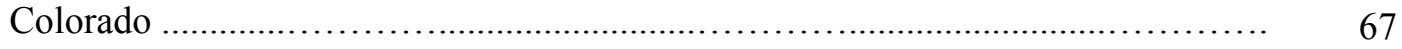

Table A-2. $\quad$ Site and Weather Data Summary for Lasvega.TM2 Weather, Las Vegas, Nevada . 68

Table A-3. Header Elements in the TMY2 Format (for First Record of Each File) ............. 71

Table A-4. Data Elements in the TMY2 Format (for All Except the First Record) .............. 71

Table A-5. Solar Radiation and Illuminance Source Flags......................................... 75

Table A-6. Solar Radiation and Illuminance Uncertainty Flags..................................... 75

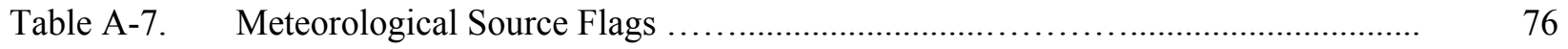

Table A-8. Meteorological Uncertainty Flags ......................................................... 76

Table B-1. Breakdown of B-EX Daily Internal Loads ............................................... 77

Table B-2. Comparison of Proposed Internal Sensible Loads With Building America 79 Prototype House Results

Table B-3. Comparison of Proposed Internal Latent Loads With Building America Prototype 79 House Results

Table C1-1. Disaggregated Interior Surface Film Coefficients ............................................ 82

Table C1-2. Interior Convective Surface Coefficients for Vertical Surfaces.............................. 84

Table C1-3. Interior Convective Surface Coefficients for Roof and Gables ............................. 84

Table C1-4. Interior Convective Surface Coefficients for Horizontal Surfaces ......................... 84

Table C1-5. Interior Convective Surface Coefficients for Single-Pane Windows ...................... 84

Table C1-6. Effect of Window Interior Surface Convective Calculation for EnergyPlus

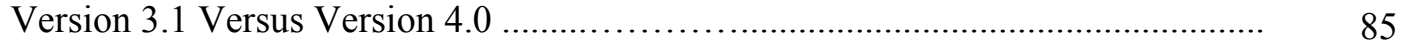

Table C2-1. Combined Exterior Heat Transfer Coefficients for Each Surface Type ................. 87

Table C2-2. Combined Exterior Heat Transfer Coefficients After Area Weighting .................. $\quad 88$

Table C2-3. Disaggregated Exterior Film Coefficients for Opaque Surfaces ............................ 89 


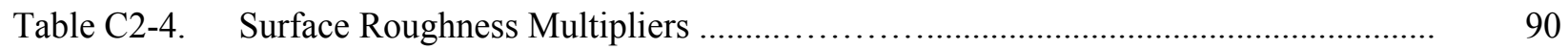

Table C2-5. Exterior Convective Surface Coefficients for Vertical Surfaces ........................... 90

Table C2-6. Exterior Convective Surface Coefficients for Roof and Gables .............................. 90

Table C2-7. Exterior Convective Surface Coefficients for the Floor ............................................ 91

Table C2-8. Exterior Convective Surface Coefficients for Windows ......................................... 91

Table D-1. EnergyPlus Infiltration Sensitivity Test Results ................................................ 94

Table F-1. Approximate Input Ranges (AIRs), Nominal Inputs, and Portions of AIRs Used for Generating Explicit Input Sets Corresponding to Low, Random, and High Space-Conditioning Energy Consumption .................................................. 103

Table G-1. $\quad$ BESTEST-EX Building Physics Heating Tests Reference Results ........................ 106

Table G-2. $\quad$ BESTEST-EX Building Physics Cooling Tests Reference Results ....................... 106

Table G-3. Benefit of Calibration $(B o C)$ for Working Group Field Trial ............................... 115

Table G-4. Benefit of Calibration for Combined Retrofit Cases (L300EX) in Working Group Field Trial ......................................................................................... 116 


\section{Introduction}

A number of computerized energy auditing systems use utility bill data and a variety of calibration methods with the objective of tuning their audit models to more accurately predict energy savings from retrofits. A potential increase in performance-based tax incentives for home energy retrofits is driving the need for establishing procedures to test the accuracy of building energy audit software used to predict retrofit energy savings. The National Renewable Energy Laboratory (NREL), in work spanning 30 years, has led development of numerous procedures for evaluating various aspects of building energy analysis computer programs used in both commercial and residential applications. Consequently, the U.S. Department of Energy (DOE) tasked NREL to develop a process for testing the reliability of models that predict retrofit energy savings, including their associated calibration methods. DOE asked NREL to conduct the work in phases so that a test procedure would be ready should DOE need it to meet legislative requirements related to residential retrofits in FY 2010. This report documents the initial "Phase 1" test procedure. NREL expects to continue to improve the test procedure as additional empirical residential energy retrofit data become available. This report has two purposes, a) to serve as a test procedure, and b) to describe the process of developing the procedure, and what was learned during the work.

\section{Overview of the BESTEST-EX Phase 1 Test Suite}

This test suite represents a set of cases applying the NREL BESTEST-EX Methodology. It includes two kinds of test cases:

Building physics test cases with fully known inputs: A given audit model is tested using specified inputs; resulting outputs are compared with reference results from three detailed simulation programs (EnergyPlus version 3.1, DOE-2.1E version JJHirsch PC 2.1En136, and SUNREL version 1.14) presented in Appendix G. Tested program results may also be compared with example acceptance criteria (Judkoff et al. 2010), or other results generated using this test procedure.

Calibrated energy savings test cases with specified base-case monthly utility bill data and uncertainty ranges for selected inputs: A given audit model (and associated calibration method) is tested by comparing utility-bill-calibrated energy savings predictions to results from the reference programs listed above. Reference results for the calibrated energy-savings tests are not published with the test procedure so that both automated and manual calibration methods are tested blind, without access to the reference results (answers). Practical application of this procedure requires that tested-program results are compared to reference results by a third-party. The calibrated energy savings tests represent a new methodological development, further described under "Methodology" below.

The cases test the ability to model space heating loads in a representative heating climate and space cooling loads in a representative cooling climate. The building physics and calibrated energy savings cases include the following retrofit cases: infiltration air sealing, attic insulation, wall insulation, programmable thermostat, low-e windows, low exterior solar-absorptance roof (cool roof), and external solar shading. Combined retrofit cases are also included as appropriate to heating and cooling climates, respectively. The cases are summarized in Table 1.

To help avoid user input errors, the input for the test cases is as simple as possible, and represents "typical" constructions and thermal and physical properties. The BESTEST-EX base building is based on HERS BESTEST (Judkoff and Neymark 1995a). Typical building descriptions and physical properties published by sources such as DOE, the National Association of Home Builders, the American Society of Heating Refrigerating and Air-Conditioning Engineers (ASHRAE), and the National Fenestration Rating Council (NFRC) are used for the test cases. The development team used empirical information from several large utility bill studies (Blasnik 2009), in consultation with industry participants (BESTEST-EX 
Working Group 2009), to modify some of the thermal inputs to be more appropriate for poorly insulated older buildings.

\section{Methodology}

NREL has developed a number of building energy simulation test (BESTEST) suites for evaluating and diagnosing errors in software used for energy analysis of residential and commercial buildings. These test suites have been adopted and cited by many organizations such as the Internal Revenue Service (2008) (for certifying software used to determine tax deductions), ASHRAE (ANSI/ASHRAE/IESNA 2007), RESNET (2006, 2007), the International Energy Agency (Judkoff and Neymark 2009), and the European Community under their Energy Performance Directive (European Union 2002). These methods include software-to-software comparative testing, verification versus analytical solutions, and validation versus vetted empirical data. The theoretical basis for the BESTEST procedures is further described in the literature (ASHRAE 2009, Judkoff 1988, Judkoff et al. 2008, Judkoff and Neymark 2006).

The building physics test cases described in the preceding section are a direct application of software-tosoftware comparative test methods. The calibrated energy savings tests required NREL to make a methodological advancement to existing comparative test methods, as follows.

\section{Introduce input uncertainty into the test specification (this represents uncertainty associated with developing inputs from audit survey data):}

a. Perform sensitivity tests on inputs with potentially high uncertainty to determine their relative effect on output; select the inputs that have the greatest effect on outputs as approximate inputs.

b. Specify uncertainty ranges (approximate input ranges) for the approximate inputs.

\section{Develop reference simulation results:}

a. Generate base-case synthetic utility bill data using the same state-of-the-art reference simulation programs as used in the building physics test cases.

i. For the reference simulations, inputs that are randomly selected from within the specified approximate input ranges are designated as explicit inputs; the reference simulation explicit inputs are not included in the test specification (kept secret)

ii. All reference simulations use the same or equivalent explicit inputs for a given calibration scenario.

b. Generate reference energy savings results by adjusting appropriate base case inputs (including explicit inputs) as specified for each retrofit case.

\section{Develop tested program results:}

a. Develop the preliminary non-calibrated base-case model for a given calibration scenario.

b. Predict energy savings by either:

i. Calibrating the base-case model inputs using the synthetic utility bills (described in $2 \mathrm{a}$ above) and then applying the specified retrofit cases to the calibrated model, or

ii. Applying the specified retrofit to the non-calibrated base case model and then calibrating or correcting energy savings predictions using the synthetic utility bills (without adjustment to base-case model inputs), or

iii. Other calibration methods. 


\section{Compare results of tested programs (and their calibration techniques) versus reference simulation base-case usage and retrofit energy savings projections:}

a. Example acceptance criteria may be used to facilitate the comparison.

The conceptual framework for this method was first proposed by Judkoff (2008) with important refinements contributed by others (Neymark and Norton 2009; Neymark et al. 2009). Development of the method was facilitated by convening a technical committee of software producers (the "BESTEST-EX Working Group") to provide help with quantifying approximate input ranges and developing tested program results (see Step $1 \mathrm{~b}$ and Step 3, respectively, above). The test procedure was developed in an iterative process that allowed improvement of the test specification during the simulation trials and helped simulation trial participants to improve their software.

In its purest form, the calibration test would be implemented without using the reference simulation programs. Instead, synthetic utility billing data would be generated with the tested program itself. Such a pure calibration test requires a) automated calibration or b) that the modeler running the calibration test does not know the explicit inputs used to develop the synthetic utility bills, implying that an additional modeler is needed.

\section{Future Work}

For further development of BESTEST-EX, NREL intends to add features that may include retrofit measures such as HVAC equipment, duct sealing, domestic hot water, lighting, appliances, foundation insulation, and others. Future test cases may include selected cross-referenced cases from HERS BESTEST and other existing test procedures. NREL also plans to address using empirical data from existing audited homes to quantify accuracy of building energy simulation tools when used for modeling older poorly insulated buildings, and retrofits to those buildings. Based on this work, refinements to BESTEST-EX to better match empirical data may also be considered. Appendix I provides more detail about recommendations for future work.

\section{Advice to Certifying Agency}

This test procedure is written so that it may be referenced directly by a certifying agency. A tested program may be thought of as successfully completing the test procedure when its results compare favorably with reference program outputs on a case-by-case and a sensitivity (difference between selected cases) basis. Example acceptance criteria based on the reference results of Appendix G, Section G.1 of this report are included in Example Procedures for Developing Acceptance-Range Criteria for BESTEST$E X$ (Judkoff et al. 2010). That document, which may also be referenced directly by a certifying agency, illustrates how a certifying agency may evaluate a software tool with BESTEST-EX. The procedure for developing example acceptance ranges is also provided there. A certifying agency using BESTEST-EX may adopt these acceptance criteria or develop their own criteria. Neither DOE, the National Renewable Energy Laboratory (NREL), nor the authors of this report can be held responsible for any misfortunes caused by the use of the BESTEST-EX test procedure or the BESTEST-EX example acceptance criteria in a certification program. 
Table 1. BESTEST-EX Case Summary

\begin{tabular}{|c|c|c|c|c|c|c|c|c|c|}
\hline \multirow[b]{2}{*}{$\begin{array}{l}\text { Case } \\
(\text { Notes 1,2,3) }\end{array}$} & \multirow[b]{2}{*}{ Test Type } & \multirow{2}{*}{$\begin{array}{l}\text { INFILTRATION } \\
\text { (ACH, } \\
\text { CSprgs / LV) }\end{array}$} & \multirow{2}{*}{\multicolumn{2}{|c|}{$\begin{array}{c}\text { R-VAL (compos.) } \\
\left(\mathrm{h} \cdot \mathrm{ft}^{2} \cdot \mathrm{F} / \mathrm{Btu}\right) \\
\text { CEILING } \mid \text { WALLS } \\
\end{array}$}} & \multicolumn{2}{|c|}{ WINDOWDATA } & \multirow{2}{*}{$\begin{array}{l}\text { Roof } \\
\text { Solar } \\
\text { Abs. }\end{array}$} & \multirow[b]{2}{*}{$\begin{array}{l}\text { TSTAT ( }{ }^{\circ} \mathrm{F}, \\
\text { CSprgs/LV) }\end{array}$} & \multirow[b]{2}{*}{ Context } \\
\hline & & & & & TYPE & $\begin{array}{l}\text { SHADE } \\
\text { (OHANG) }\end{array}$ & & & \\
\hline L200EX & Base & $0.760 / 0.492$ & 13.7 & $\overline{5.1}$ & SATB & $\mathrm{NO}$ & 0.6 & $68 / 78$ & "-P", "-C" \\
\hline L210EX & Infl & $0.382 / 0.246$ & 13.7 & 5.1 & SATB & $\mathrm{NO}$ & 0.6 & $68 / 78$ & "-P", "-C" \\
\hline L220EX & Attic Ins & $0.760 / 0.492$ & 42.7 & 5.1 & SATB & $\mathrm{NO}$ & 0.6 & $68 / 78$ & "-P", "-C" \\
\hline L225EX & Wall Ins & $0.760 / 0.492$ & 13.7 & 13.0 & SATB & $\mathrm{NO}$ & 0.6 & $68 / 78$ & "-P", "-C" \\
\hline L240EX & Tstat & $0.760 / 0.492$ & 13.7 & 5.1 & SATB & NO & 0.6 & \begin{tabular}{|l|}
$68-62 I$ \\
$78-84$
\end{tabular} & "-P", "-C" \\
\hline L250EX & Low-e win & $0.760 / 0.492$ & 13.7 & 5.1 & DLEW & $\mathrm{NO}$ & 0.6 & $68 / 78$ & "-P", "-C" \\
\hline \begin{tabular}{|l} 
L260EX-P \\
\end{tabular} & $\begin{array}{l}\text { RoofAbs1 } \\
\end{array}$ & $0.760 / 0.492$ & 13.7 & 5.1 & SATB & $\mathrm{NO}$ & 0.8 & $68 / 78$ & "-P" only \\
\hline L265EX & RoofAbs2 & $0.760 / 0.492$ & 13.7 & 5.1 & SATB & $\mathrm{NO}$ & 0.2 & $68 / 78$ & $\begin{array}{l}\text { "--P" htg\&clg, } \\
\text { "-C" clg only }\end{array}$ \\
\hline L270EX-P & Ext. Shade & $0.760 / 0.492$ & 13.7 & 5.1 & SATB & S+E/W & 0.6 & $68 / 78$ & "-P" only \\
\hline $\begin{array}{l}\text { L300EX-PH } \\
\text { L300EX-CnH }\end{array}$ & Combined & $0.382 / 0.246$ & 42.7 & 13.0 & DLEW & $\mathrm{NO}$ & 0.6 & $68-62$ & $\begin{array}{l}\text { "-P" htg only } \\
\text { "-C" htg only }\end{array}$ \\
\hline L300EX-PC & Combined & $0.382 / 0.246$ & 42.7 & 13.0 & DLEW & S+EW & 0.2 & 78-84 & "-P" clg only \\
\hline [300EX-CnC & Combined & $0.382 / 0.246$ & 42.7 & 13.0 & DLEW & $\mathrm{NO}$ & 0.2 & 78-84 & "-C" clg only \\
\hline
\end{tabular}

Note 1: Changes to Case L200EX are highlighted with bold font.

Note 2: Nominal input values for "-P" cases are shown here. "-C" cases replace key nominal input values with approximate input ranges.

Note 3: "n" in case designator (e.g., "L300EX-CnH") indicates the calibrated energy savings case scenario number.

BESTEST-EX_CASES-020210.xls, B-EX(4)!a5:128

Abbreviations for Table 1:

"-C"

CSprgs

clg

DLEW

Ext

$\mathrm{E} / \mathrm{W}$

htg

htg\&clg

Infl

Ins

Low-e win

LV

OHANG

"-P"

RoofAbs1

RoofAbs 2

R-VAL (compos.)

$\mathrm{S}$

SATB

TSTAT

68-62

$78-84$ calibrated energy savings test cases

Colorado Springs, Colorado

cooling

double-pane, low-e window with wood frame and insulated spacer

exterior

east/west

heating

heating and cooling

infiltration

insulation

low-emissivity window

Las Vegas, Nevada

overhang

building physics test cases

roof with high exterior solar absorptance

roof with low exterior solar absorptance

composite air-to-air R-value

south

single-pane window with aluminum frame and thermal break

thermostat

$68^{\circ} \mathrm{F}$ heating base set point with $62^{\circ} \mathrm{F}$ set point for specified times

$78^{\circ} \mathrm{F}$ cooling base set point with $84^{\circ} \mathrm{F}$ set point for specified times 


\subsection{Test Procedures \\ $1.1 \quad$ Modeling Approach}

This modeling approach shall apply to all test cases presented in Sections 1.2 and 1.3.

\subsubsection{Time Convention}

All references to time in this specification are to local standard time and assume that hour $1=$ the interval from midnight to 1:00 a.m. Do not use daylight saving time or holidays for scheduling. Typical Meteorological Year 2 (TMY2) weather data are in hourly bins corresponding to local standard time.

\subsubsection{Geometry Convention}

If the program being tested includes the thickness of walls in a three-dimensional definition of the building geometry, then wall, roof, and floor thicknesses shall be defined such that the interior air volume of the building model remains as specified. Make the thicknesses extend exterior to the currently defined internal volume.

\subsubsection{Nonapplicable Inputs}

In some instances the specifications will include input values that do not apply to the input structure of the program being tested. When this occurs, disregard the nonapplicable inputs and continue. Such inputs are in the specifications for programs that may need them.

\subsubsection{Consistent Modeling Methods}

Where there are options in a simulation program for modeling a specific thermal behavior, consistent modeling methods shall be used for all cases. For example, if a software program provides a choice of methods for modeling windows, use the same window modeling method for all cases.

\subsubsection{Equivalent Modeling Methods}

Where a program or specific model in a program does not allow direct input of specified values, or where input of specified values causes instabilities in a program's calculations, modelers should develop equivalent inputs that match the intent of the test specification as nearly as the software being tested allows. Such equivalent inputs are to be developed based on the data provided in the test specification, and such equivalent inputs shall have a mathematical, physical, or logical basis, and shall be applied consistently throughout the test cases.

\subsubsection{Simulation Initialization and Preconditioning}

If the program being tested allows, begin the simulation initialization process with zone air conditions that equal the outdoor air conditions. If the program being tested allows for preconditioning (iterative simulation of an initial time period until temperatures or fluxes, or both, stabilize at initial values), use that capability.

\subsubsection{Simulation Duration}

Results for the tests in Section 1 are to be taken from a full annual simulation.

\subsubsection{Programs With Different Operational Modes Depending on Utility Data Availability}

If the software being tested applies a different mode for running the building physics test cases (see Section 1.2) than for running the calibrated energy savings test cases (see Section 1.3)-i.e., when no utility billing data are available, versus when utility data are available - use the appropriate program mode corresponding to the specific test type; apply it consistently for the given test type. 


\subsubsection{Order of Testing}

The BESTEST-EX test suite has two main sections corresponding to two different types of test cases:

- "Building Physics" test cases (Section 1.2)

- "Utility Bill Calibration" test cases (Section 1.3).

\subsubsection{Building Physics Test Cases}

Start by running the building physics tests cases in Section 1.2. Building physics test case results may be compared to the reference simulation results provided in Appendix G (see Section G.1). Tested program results may also be compared with example acceptance criteria (Judkoff et al. 2010), or with other results generated using this test procedure. Diagnose disagreements and correct modeling errors before moving on to Section 1.3. Correction of modeling errors must have a mathematical, physical, or logical basis and must be applied consistently throughout the test cases. Some disagreements may have a logical basis (i.e., may be based on legitimate modeling differences).

\subsubsection{Calibrated Energy Savings Test Cases}

Next, run the calibrated energy savings test cases of Section 1.3. Section 1.3 is written such that a) a preliminary non-calibrated base-case model is developed as described in Section 1.3.1, b) inputs for the base-case simulation model (see Section 1.3.1) are calibrated using synthetic reference utility energy-use data given in Section 1.3.1.2, and c) inputs for retrofit cases (see Section 1.3.2) are developed using calibrated base-case inputs with modifications as specified for the given retrofit cases. Some modeling methods may calculate calibrated energy savings, without adjustment to model inputs, e.g., by comparing differences between base case utility billing data versus predicted non-calibrated base-case energy use, and then applying an appropriate adjustment to predicted non-calibrated energy savings. For programs that apply methods not requiring adjustment to base-case model inputs, use the utility bills called out in Section 1.3.1.2 for calibration; however, specific instructions of Section 1.3.1.2 (and elsewhere in Section 1.3) regarding adjustment of inputs for calibration do not apply.

Reference results for the calibrated energy-savings tests are not published with the test procedure, so that both automated and manual calibration methods may be tested blind, without access to the reference results. Practical application of this procedure requires that tested-program results are compared to reference results by a third-party.

\subsection{Building Physics Test Input Specifications}

The test cases are described in a manner that allows many different residential modeling tools, representing different degrees of modeling complexity, to be tested. Within this structure, figures and tables are grouped as summary data and supplemental data. The summary data, which are based on the supplemental data, are figures and tables that contain information that summarizes most of the input requirements for most users. The supplemental tables contain more detailed information that was required for generating a consistent set of inputs to the reference programs. Such data include material properties for modeling thermal mass and modeling the attic as a separate zone, interior solar distribution fractions, combined convective and radiative surface coefficients, hourly internal gains schedules, and detailed window optical properties. Use the supplemental data as needed, according to the inputs allowed by the tool being tested.

Apply the modeling rules of Section 1.1 for all test cases. Abbreviations used in the tables, figures, and text are defined on the acronyms and abbreviations page included with the front matter. 


\subsubsection{The Pre-Retrofit Base-Case Building (Case L200EX-P)}

Begin with Case L200EX-P. Case L200EX-P shall be modeled as detailed in this section and its subsections. HERS BESTEST (Judkoff and Neymark 1995a) Case L200A is the basis for Case L200EX-P.

A major part of the work for implementing the tests is assembling an accurate base building model. Double-check base building inputs before addressing the retrofit cases.

\subsubsection{Weather Data}

This case requires the use of both the Colorad.TM2 and Lasvega.TM2 weather data provided with accompanying files. These data are used for heating-only and cooling-only test cases, respectively, per Section 1.2.1.14. If the program being tested uses a different representation of weather, such as degree days, bin method, etc., then the above weather data shall be processed with the tested program's weather data processor so its output will be based on the above data. A summary of the data and a description of TMY2 (.TM2) weather data format are provided in Appendix A.

\subsection{Ground Reflectance}

The solar reflectance of the site ground surface $=0.2$.

\subsubsection{Output Requirements}

Output requirements are the same for all test cases. Use the output template BESTEST-EX-Phase-1Output.XLS, included with the accompanying electronic files, to enter monthly utility data (metered energy use) for a full-year simulation for the program being tested. Provide monthly natural gas consumption in million $\left(10^{6}\right) \mathrm{Btu}$, and electricity consumption in $\mathrm{kWh}$. Monthly billing periods are assumed to run from the first day of the month to the last day of the month: e.g., January 1-31, February 1-28. Results sets for heating and cooling building physics test cases are designated as LnnnEX-PH and LnnnEX-PC, respectively. Further instructions are included with the output template.

If the software being tested does not include domestic hot water (DHW) in its analysis, develop gas DHW consumption using an external calculation (e.g., spreadsheet) and include the externally calculated DHW consumption with the tested program's calculated space heating consumption in the total gas utility bill.

\subsubsection{Building Geometry and Material Properties}

The base building plan is a $1,539 \mathrm{ft}^{2}$ floor area, single-story house with one conditioned zone (the main floor), an unconditioned attic, and a raised floor exposed to air. Note the following regarding information provided in figures and tables.

- For the building physics tests use only "Nominal" Inputs provided in the tables; approximate input ranges ("Min" and "Max" values) are for use with Section 1.3.

- Changes to HERS BESTEST Case L200A are highlighted with bold font in figures and tables.

The following figures and tables contain information that is applicable to most users. Insulation R-values noted in the figures are nominal values; use the tables for finding appropriate inputs.

Figure 1-1. Base building axonometric

Figure 1-2. Floor plan - Case L200EX

Figure 1-3. East side elevation - Case L200EX

Figure 1-4. Exterior wall plan section - Case L200EX

Figure 1-5. Raised floor exposed to air section - Case L200EX

Figure 1-6. Ceiling/attic/roof section - Case L200EX 
Figure 1-7. Interior wall plan section - Case L200EX

Figure 1-8. Window detail, vertical slider (NFRC AA) with 23/4" wide frame - Case L200EX

Table 1-1. Building Thermal Summary - Case L200EX

Table 1-2. Other Building Details - Case L200EX.

Relevant supplementary tables that include more detailed information are:

Table 1-3. Component Surface Areas and Solar Fractions - Case L200EX

Table 1-4. Material Descriptions, Exterior Wall, Door, and Window - Case L200EX

Table 1-5. Material Descriptions, Raised Floor Exposed to Air - Case L200EX

Table 1-6a. Material Descriptions, Ceiling, Attic, and Roof - Case L200EX

Table 1-6b. Material Descriptions, Ceiling/Attic/Roof, Attic as Material Layer -

Case L200EX (for calculating equivalent ceiling/attic/roof composite R-value)

Table 1-7. Material Descriptions, Interior Wall - Case L200EX

Table 1-8a. Conditioned Zone Equivalent Inputs for Weather-Driven Infiltration

Table 1-8b. Equivalent Seasonal Constant Infiltration ACH and CFM - Case L200EX

Table 1-9a. Daily Sensible Internal Loads - Case L200EX

Table 1-9b. Normalized Hourly Profiles for Sensible Internal Loads - Case L200EX

Table 1-10. Window Summary (Single-Pane Aluminum Frame With Thermal Break) Case L200EX

Table 1-11. Glazing Summary, Single-Pane Center of Glass Values - Case L200EX

Table 1-12. Optical Properties as a Function of Incidence Angle for Single-Pane Glazing Case L200EX.

Other details not described in these figures and tables are discussed topically in the following subsections.

\subsubsection{Attic}

Many residential energy analysis tools input an attic by specifying it within a menu of roof types, and then specifying the insulation-only R-value corresponding to the insulation installed on the attic floor. If this is the case for the software being tested, the information provided in Figure 1-6 will be sufficient.

For programs such as those used for developing the reference results, more detailed information is required. The detailed information for modeling an attic as a separate zone is supplied in Table 1-6a. Table 1-6b gives similar information as Table 1-6a, except in Table 1-6b the attic space is modeled as a layer of thermal resistance between ceiling and roof materials. In the tables the modeled joist thickness is the same as that for the batt insulation (3.5 in.); the joists' remaining height above the insulation is assumed to be at the attic air temperature and is not considered as thermal mass.

Table 1-6b documents the calculation of ceiling/attic/roof composite air-air R-value noted in the building thermal summary of Table 1-1. In Table 1-6b, the equivalent resistance for the attic is based on values from the Cooling and Heating Load Calculation Manual (McQuiston and Spitler 1992, p. 4.12); typical ventilation by natural effects and roof solar absorptance of 0.6 were assumed. EnergyPlus preliminary sensitivity test results for the two-zone model versus the one-zone model indicate only a $0.2 \%$ difference in heating load results for Colorado Springs and 0.6\% cooling load results for Las Vegas.

As with other components - except where explicitly varied by the test specification - the attic must be modeled consistently for all test cases such that the modeling rules of Section 1.1 are applied. 


\subsubsection{Raised Floor Exposed to Air}

The raised floor exposed to air is provided as an idealization, because when HERS BESTEST was developed the state-of-the-art for modeling heat transfer between the house and the ground was not very advanced in whole-house simulation models. Such building-to-ground heat transfer occurs for slab-ongrade, basement, and crawl space constructions. To somewhat decouple the floor from the modeling problem, the raised floor is provided with R-19 batt insulation. To simulate a raised floor exposed to air, the test cases require the following assumptions:

- Air temperature below the raised floor is assumed to equal outdoor air temperature.

- The underside of the conditioned zone floor has an equivalent combined convective and radiative exterior film coefficient of $2.200 \mathrm{Btu} /\left(\mathrm{h} \cdot \mathrm{ft}^{2} \cdot{ }^{\circ} \mathrm{F}\right)$, consistent with a "rough" surface texture and zero wind speed (see Appendix C); if the program being tested cannot set the exterior surface coefficient to a fixed value, allow the exterior surface coefficient to vary with wind speed. If the tested program allows detailed designation of different surface heat transfer algorithms among specific surfaces, apply a surface convection algorithm that incorporates only surface-to-air temperature difference (excludes wind or sets wind speed $=0$ ), and include infrared radiative exchange separately.

- The conditioned zone floor exterior surface (surface facing downward) receives no solar radiation.

The assumption of the air temperature below the raised floor being equal to ambient temperature may be approximated either by modeling a building that hovers above the ground (raised floor on stilts for example), or modeling a highly ventilated crawl space. The zero solar-radiation-to-exterior-floor assumption can be modeled by assigning the highest solar reflectance allowed by the software being tested to the underside of the floor and/or defining shading planes where walls would be if the raised floor were modeled as a crawl space. Infrared radiative exchange between the conditioned zone floor exterior surface (surface facing downward) and the ground surface (assumed at ambient air temperature) is modeled in the EnergyPlus and DOE-2.1E reference simulations; SUNREL applies the specified combined surface coefficient.

\subsubsection{Interior Walls}

The interior walls in the conditioned zone are included for modeling the effect of their mass. They are not intended to divide the conditioned zone into separately controlled zones.

\subsubsection{Infiltration}

\subsection{Conditioned Zone}

Infiltration is modeled assuming blower door data are available for the pre-retrofit base case (L200EX-P). Detailed inputs for programs that apply Sherman-Grimsrud infiltration modeling are provided in Table 1-8a. Use only the inputs that apply to the software being tested. For programs that do not apply Sherman-Grimsrud modeling, values for equivalent seasonal constant air changes per hour (ACH) (or cubic feet per minute [CFM]) are included in Table 1-8b. These equivalent constant values are also included as part of the building overview description in summary Table 1-2; these are also used for developing building summary UA characteristics in Table 1-1.

The equivalence of the inputs of Table 1-8a is based on the ASHRAE residential air leakage model (2005 ASHRAE Handbook of Fundamentals, pp. 27.12, 27.13, 27.21), which is based on the model developed by Sherman and Grimsrud (1980). Supporting information for ASHRAE residential air leakage model inputs used to generate reference simulation results is included in Appendix D. Appendix D also includes discussion of some other approaches to modeling infiltration given the results of fan pressurization (blower door) tests, along with development of the equivalent constant infiltration rates. 
The Colorad.TM2 and Lasvega.TM2 climate sites are at 6,171 ft and 2,178 ft altitude, respectively, so the air is less dense than that at sea level for both locations. If the program being tested applies a constant infiltration rate only and does not use barometric pressure from the weather data, or otherwise does not automatically correct for the change in air density caused by altitude, adjust the constant specified infiltration rates (to yield mass flows equivalent to what would occur at the specified altitude), as shown in Table 1-8b.

\subsection{Attic}

Use the constant attic infiltration rate given in Table $1-8 \mathrm{~b}$ only if the software being tested allows that input. Equivalent Sherman-Grimsrud model inputs were not developed for the attic. Attic infiltration is based on the Cooling and Heating Load Calculation Manual (McQuiston and Spitler 1992) for typical ventilation by natural effects. The calculation technique used for developing altitude effects on infiltration is included in HERS BESTEST Appendix B.

\subsubsection{Internal Loads}

All internal loads data are new for this test specification; i.e., changed from HERS-BESTEST. These are non-HVAC related internally generated loads in the conditioned zone from equipment, lights, people, etc. The internal loads schedules disaggregate sensible and latent loads. Internal loads are further disaggregated by associated fuel type, where internal loads related to DHW are associated with gas use and all other non-HVAC related internal loads are associated with electricity use. There are no internal loads in the attic. The selection of sensible and latent internal loads and the development of schedules are described in Appendix B. Details about internal loads are provided below; summary data are given in Table 1-2.

If the software being tested does not include DHW in its analysis, develop gas DHW consumption using an external calculation (e.g., spreadsheet) and include the externally calculated DHW consumption with the tested program's calculated space heating consumption in the total gas utility bill.

\subsection{Sensible Loads}

Nominal values for daily total sensible internal loads disaggregated for occupants, electricity, and gas are specified in Table 1-9a. Normalized sensible load hourly profile fractions for the conditioned zone are specified in Table 1-9b; the hourly fractions apply for all days of the year as given.

\subsection{Radiative and Convective Fractions}

Sensible loads are $70 \%$ radiative and $30 \%$ convective.

\subsection{Latent Loads}

Modeling latent loads for space cooling requires assumptions about the moisture removal by a mechanical space cooling condensing unit. For the Phase 1 Test Procedure latent loads are not applied. This is because the currently specified idealized equipment for sensible cooling (see Sections 1.2.1.13 - 1.2.1.15) does not give guidance about latent load removal. For developing reference simulation results, latent loads were not included, as they have no effect on the results.

\subsection{Fractions of Base Load Usages to Internal Gains}

Only a fraction of the non-HVAC energy from electricity and gas used in a home is converted to sensible internal gains. To generate synthetic utility bills, percentages of non-HVAC gas energy and electric energy converted to sensible loads must be assumed. The following nominal values are used for conversion of non-HVAC energy use to sensible internal gains:

- $\mathbf{7 5 \%}$ of the non-HVAC energy for electric appliances and lights

- $\mathbf{2 7 . 5} \%$ of the non-HVAC energy for gas DHW. 
These values were developed in consultation with the BESTEST-EX Working Group (2009). Further background discussion is included in Appendix B. Resulting nominal non-HVAC energy usage based on these fractions is included in Table 1-9a.

Reference simulations integrate internal gains by applying internal gains fractions for electricity (X\%) and gas (Y\%) using the following steps:

1. Convert the sensible internal gains due to non-HVAC electric appliances and lights (divide by $\mathrm{X} \% / 100)$ to obtain base load electricity consumption.

2. Convert the sensible internal gains due to non-HVAC gas appliances (DHW) (divide by Y\%/100) to obtain base load (DHW) gas consumption.

3. Each month:

a. Add the non-HVAC electricity consumption to the monthly HVAC electricity consumption and

b. Add the non-HVAC gas consumption to the monthly HVAC gas consumption.

4. Use $\mathrm{kWh}$ for metered electricity consumption and million $\left(10^{6}\right)$ Btu for metered natural gas consumption.

\subsubsection{Combined Radiative and Convective Surface Heat Transfer Coefficients}

If the program being tested does not allow variation of combined surface coefficients, or if it automatically calculates interior and exterior surface convection and radiation, this section may be disregarded.

Combined surface coefficients are denoted in various section drawings throughout Section 1 as "Interior Film" and "Exterior Film" (e.g., see Figures 1-4 through 1-7). If the program being tested uses combined surface coefficients, use the information given in Table 1-2; this information is also included with the detailed material descriptions (e.g., see Tables 1-4 through 1-7).

ASHRAE Terrain Class 2 (suburban/urban terrain per ASHRAE [2005, p. 16.3]) is assumed. See Appendix $\mathrm{C}$ for more information about surface coefficients.

\subsubsection{Opaque Surface Radiative Properties}

These properties apply to all opaque exterior and interior building surfaces; they are roughly equivalent to medium color paint or a light color roof.

The nominal value for exterior surface solar absorptance is 0.6. All other opaque surface radiative properties have explicit inputs, as shown in Table 1-2.

\subsubsection{Windows}

A great deal of information about the window properties has been provided so equivalent input for windows is possible for many programs. Use only the information (nominal values) relevant to the program being tested. The basic properties of the single-pane window, including shading coefficient (SC), solar heat gain coefficient (SHGC), and thermal resistance, are provided in Table 1-1. Additional information is included in Figure 1-8, Table 1-4, and Tables 1-10 through 1-12. This information was drawn primarily from the WINDOW 5.2 (2005) software for developing detailed glazing properties (see Appendix E). For programs that need transmittance or reflectance at other angles of incidence, interpolate between the values of Table 1-12 using the cosine of the incidence angle as the basis of interpolation. Where other unspecified data are needed, values that are consistent with those quoted must be calculated.

For the base case, total glass and frame areas for each wall may be combined into a single large area for that wall. For more detailed models, exterior surface convective coefficients may vary with the height of the surface centroid. 


\subsubsection{Interior Solar Distribution}

If the program being tested does not allow for variations of interior solar distribution, this section may be disregarded. Interior solar distribution is the fraction of transmitted solar radiation incident on specific surfaces in a room. If the program being tested does not calculate this effect internally, use the interior solar fractions from Table 1-3. The calculation of transmitted solar radiation reflected back out through windows (cavity albedo) is presented in HERS BESTEST Appendix E.

\subsubsection{Mechanical System}

This mechanical system applies to the conditioned zone only; it does not apply to the unconditioned attic. The mechanical system shall be modeled with the following features as noted below and in Sections 1.2.1.14 and 1.2.1.15:

- $100 \%$ convective air system

- The thermostat senses only the air temperature

- Nonproportional type thermostat (see Section 1.2.1.14)

- No latent heat extraction.

\subsubsection{Thermostat Control Strategies}

Seasonal thermostat control settings are shown for heating and cooling climates in Sections 1.2.1.14.1 and 1.2.1.14.2, respectively.

\subsection{Colorad.TM2}

For Colorad.TM2 weather data (heating only)

During heating season (October 7-May 16):

$$
\text { HEAT }=\text { ON IF TEMP }<68^{\circ} \mathrm{F} ; \mathrm{COOL}=\mathrm{OFF}
$$

During non-heating season (May 17-October 6):

$$
\mathrm{HEAT}=\mathrm{OFF} ; \mathrm{COOL}=\mathrm{OFF} \text {. }
$$

Where: "TEMP” refers to conditioned zone-air temperature.

The designated heating season is the time period during which approximately $95 \%$ of the total heating load occurs as indicated by an EnergyPlus simulation of Case L200EX-P.

\subsection{Lasvega.TM2}

For Lasvega.TM2 weather data (cooling only)

During cooling season (March 28-October 28):

$$
\mathrm{COOL}=\mathrm{ON} \text { IF TEMP }>78^{\circ} \mathrm{F} ; \mathrm{HEAT}=\mathrm{OFF}
$$

During non-cooling season (October 29-March 27):

$$
\mathrm{COOL}=\mathrm{OFF} ; \mathrm{HEAT}=\mathrm{OFF} \text {. }
$$

Where: "TEMP” refers to conditioned zone-air temperature.

The designated cooling season is the time period during which approximately $95 \%$ of the total cooling load occurs as indicated by an EnergyPlus simulation of Case L200EX-P. 


\subsection{Nonproportional Thermostat}

The thermostat is nonproportional in the sense that when the conditioned zone-air temperature exceeds the thermostat cooling set point, the heat extraction rate is assumed to equal the maximum capacity of the cooling equipment. Likewise, when the conditioned zone-air temperature drops below the thermostat heating set point, the heat addition rate equals the maximum capacity of the heating equipment. A proportional thermostat throttles the heat addition rate (or extraction rate) in proportion to the difference between the zone set point temperature and the actual zone temperature. If the program being tested requires use of a proportional thermostat, a proportional thermostat model can be made to approximate a nonproportional thermostat model by setting a very small throttling range (the minimum allowed by the program being tested).

\subsubsection{Equipment Characteristics}

HEATING CAPACITY $=3.413$ million Btu/h (effectively infinite) EFFECTIVE HEATING EFFICIENCY $=70 \%$

COOLING CAPACITY $=3.413$ million Btu/h (effectively infinite) EFFECTIVE COOLING COEFFICIENT OF PERFORMANCE $=3.0$

\section{FAN POWER $=0 \mathrm{~W}$ (no fan electricity use) \\ WASTE HEAT FROM FAN $=0 \mathrm{~W}$.}

Equipment efficiency is constant: independent of part loading, indoor dry-bulb temperature and humidity ratio, outdoor dry-bulb temperature and humidity ratio, and/or other conditions. The heating efficiency may be thought of as the ratio of heat provided to the space by the furnace divided by the furnace gas use measured at the meter, and includes all losses associated with furnace efficiency, air distribution, etc. Similarly, the cooling coefficient of performance (COP) may be thought of as the ratio of sensible heat extraction from the space by the space cooling equipment divided by the electricity use measured at the meter, and includes all losses associated with system efficiency, air distribution, etc.; latent load is not considered.

The 3.413 million Btu/h requirement comes from the IP units equivalent of $1 \mathrm{MW}$. If the software being tested does not allow this much capacity, use the largest system it allows.

The intent of the very high equipment heating and cooling capacities is to produce only pure heating load and sensible cooling load outputs by assuring that the zone load is always met, and that the zone air temperature is always maintained at the appropriate thermostat set point (or within the minimum throttling range allowed by the program being tested) when either heating or cooling is required. If 3.413 million Btu/h of capacity causes the simulation program being tested to become unstable, then use a smaller value for over-sizing equipment, but not less than the capacity required to maintain the set point temperature for each case. 


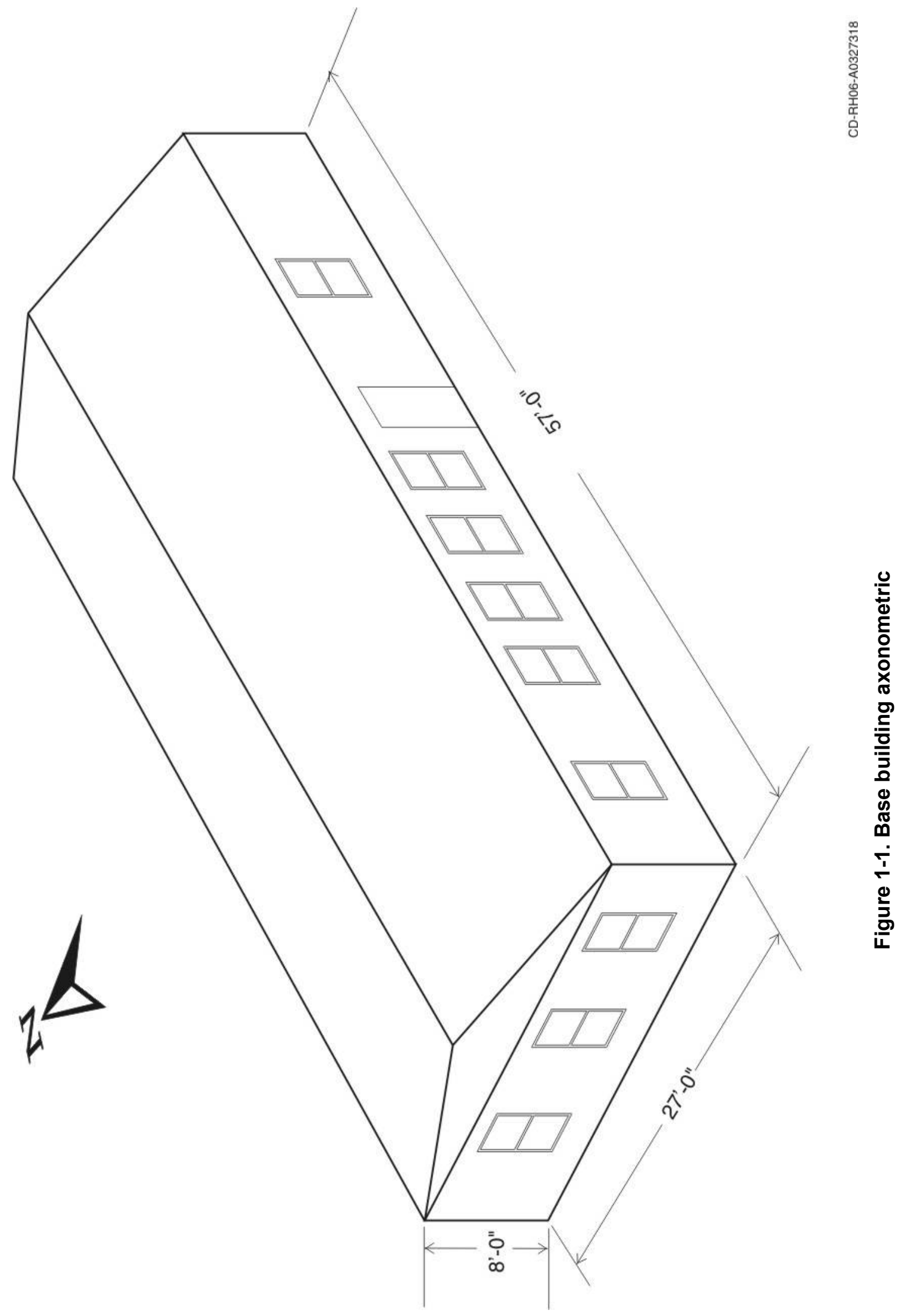




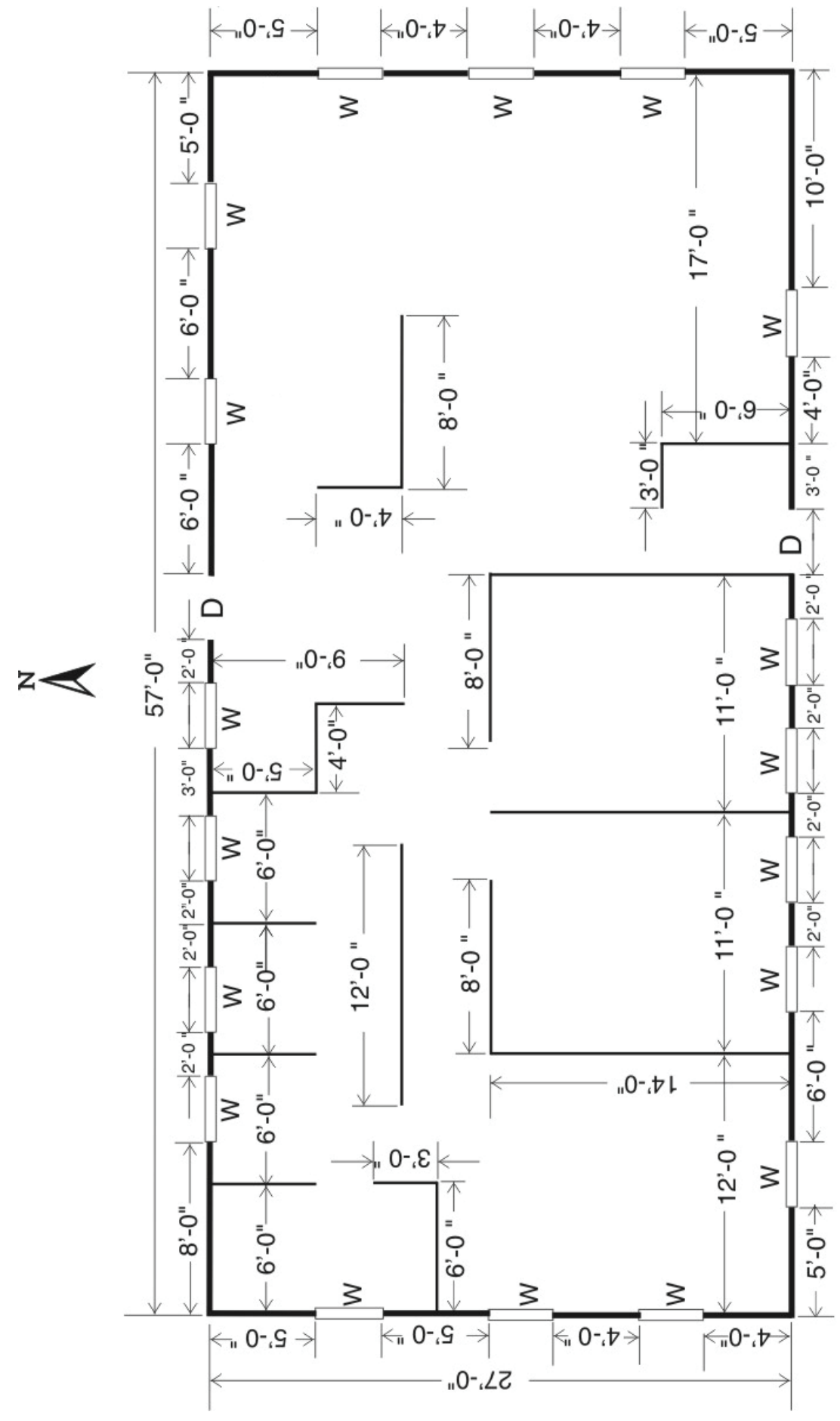

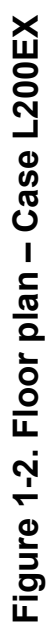




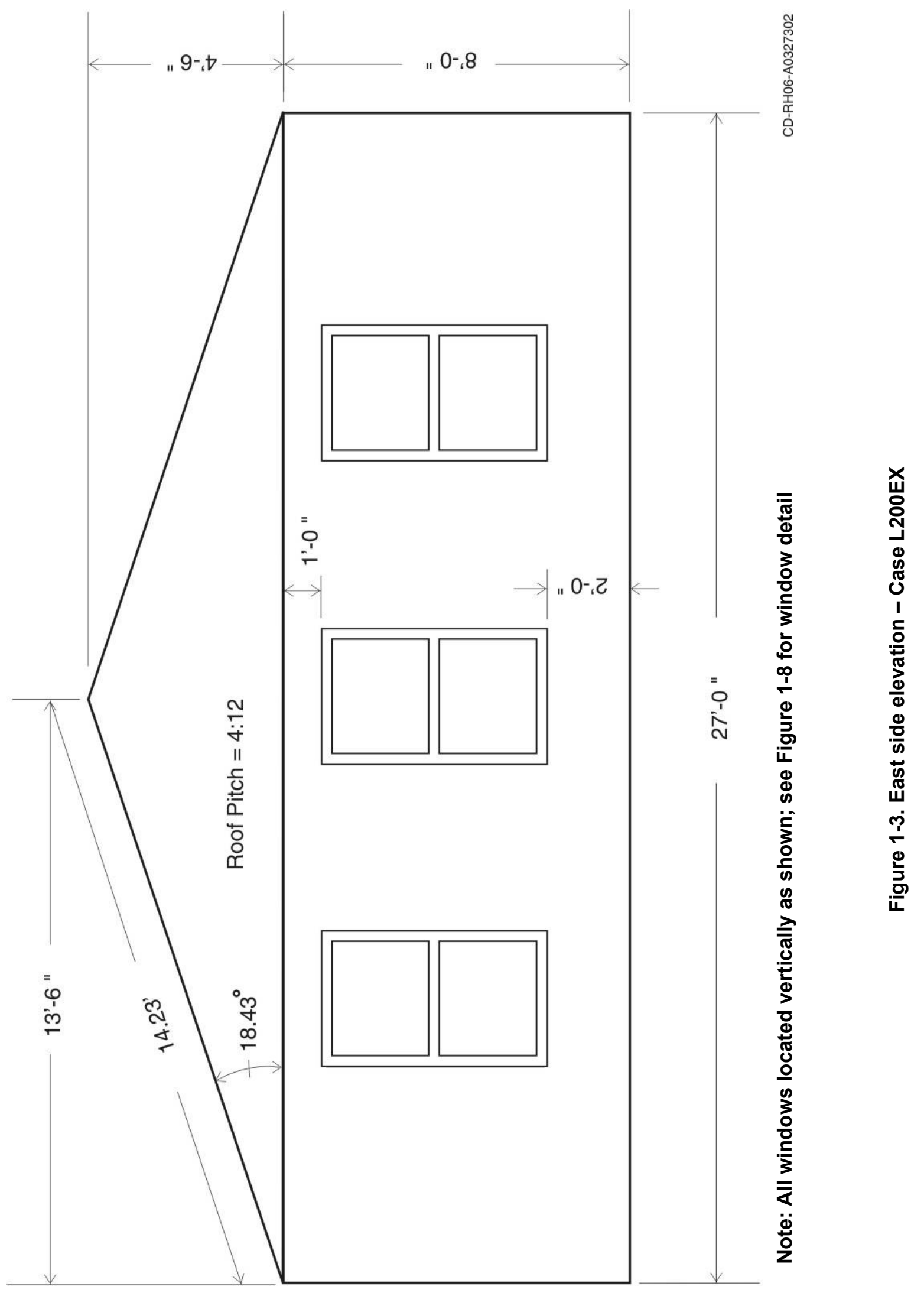




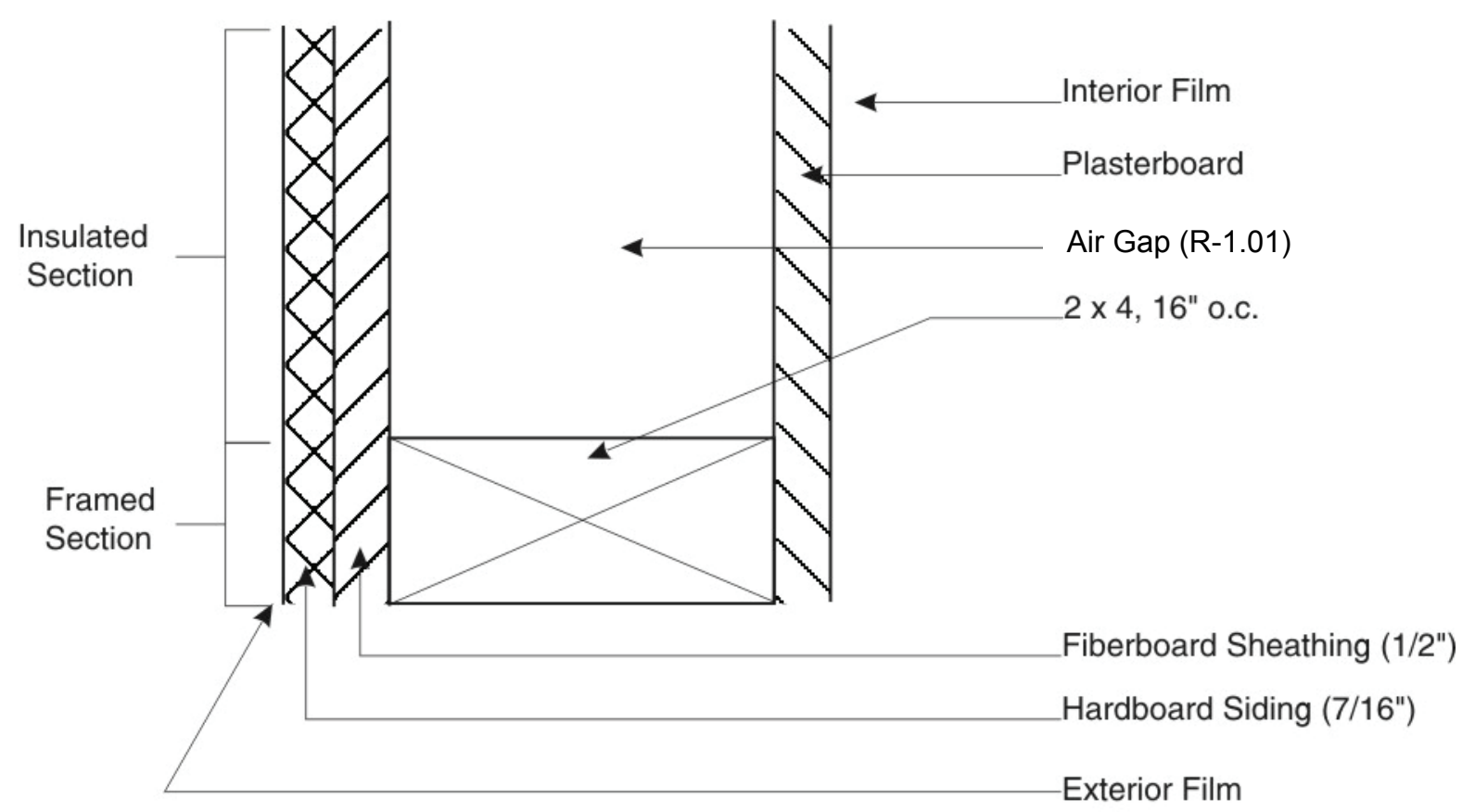

CD-RH06-A0327319A

Figure 1-4. Exterior wall plan section - Case L200EX

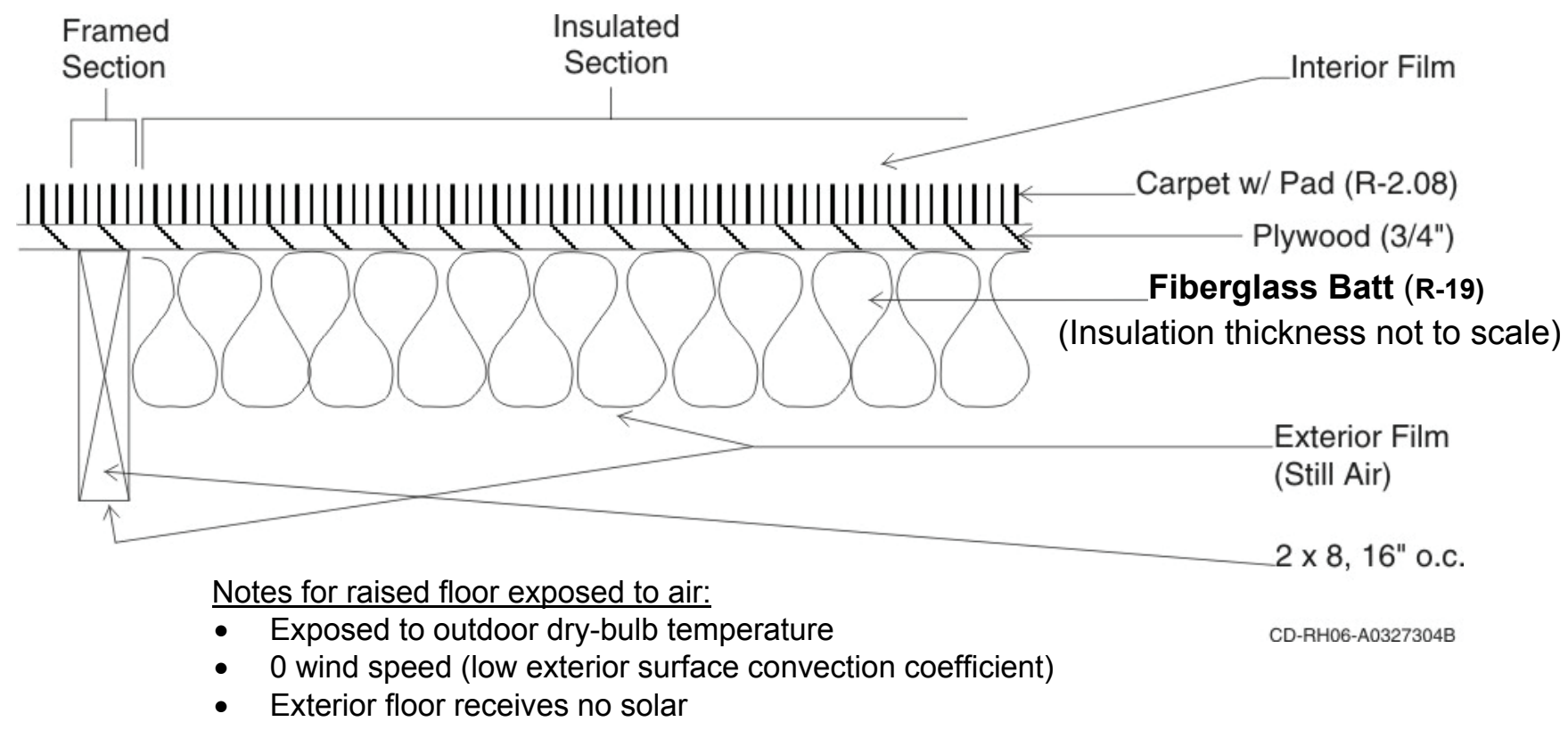

Figure 1-5. Raised floor exposed to air section - Case L200EX 


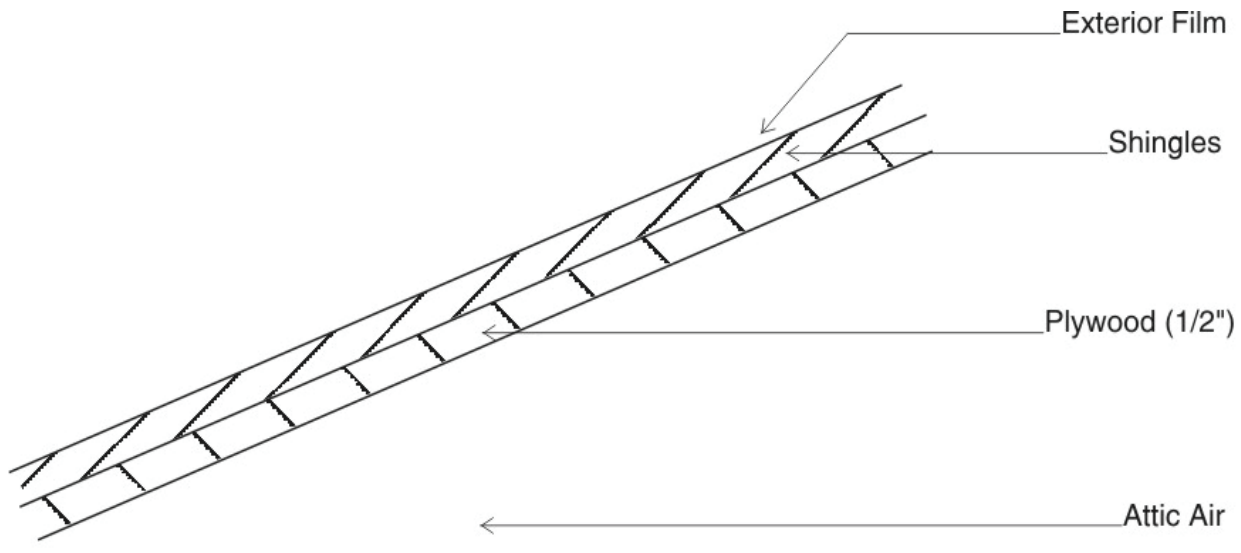

$2 \times 6,24 "$ o.c.

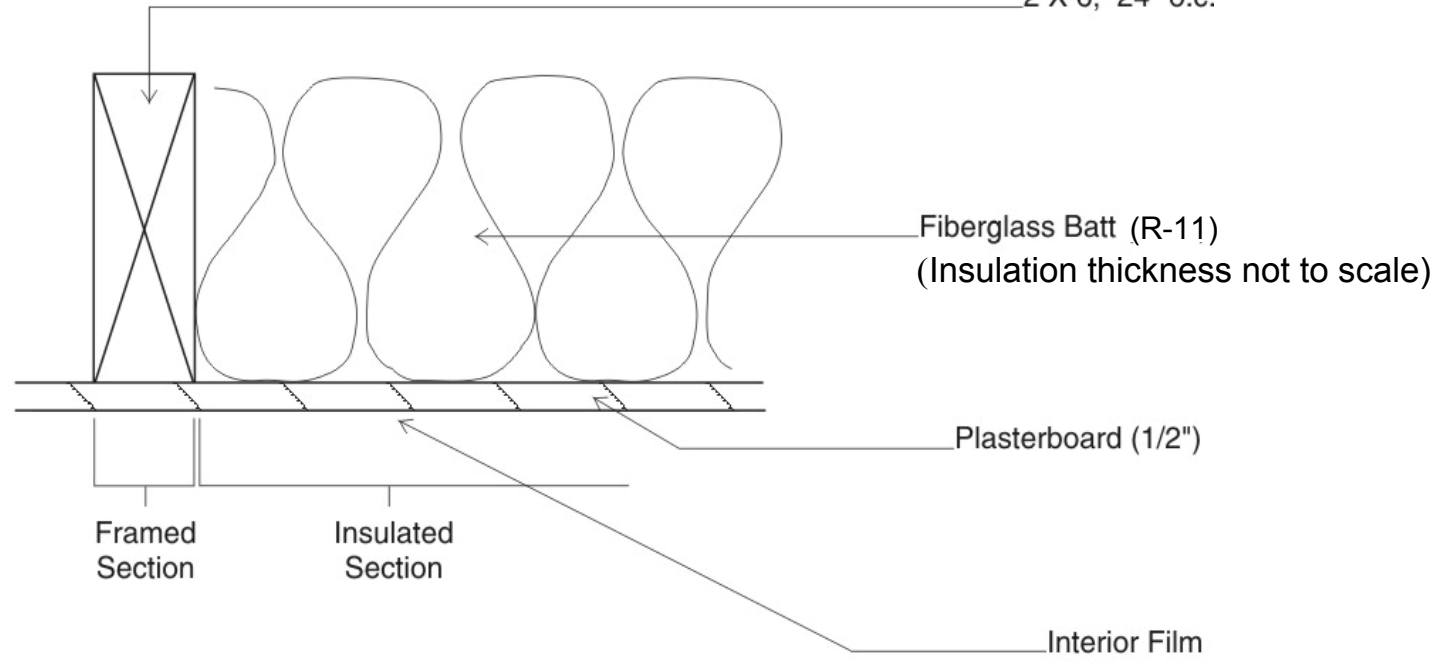

CD-RH06-A0327305

Figure 1-6. Ceiling/attic/roof section - Case L200EX 


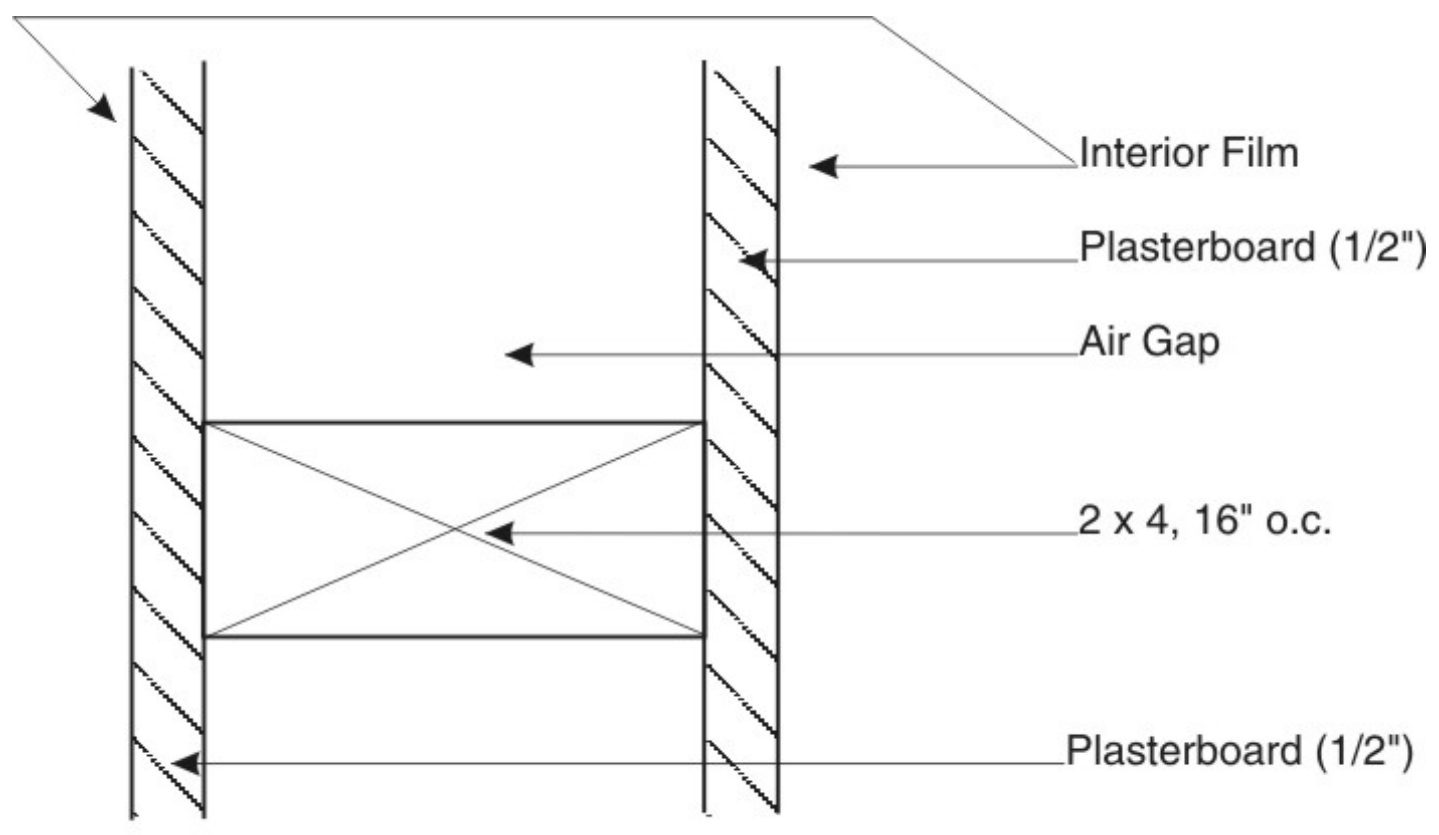

CD-RH06-A0327323

Figure 1-7. Interior wall plan section - Case L200EX 


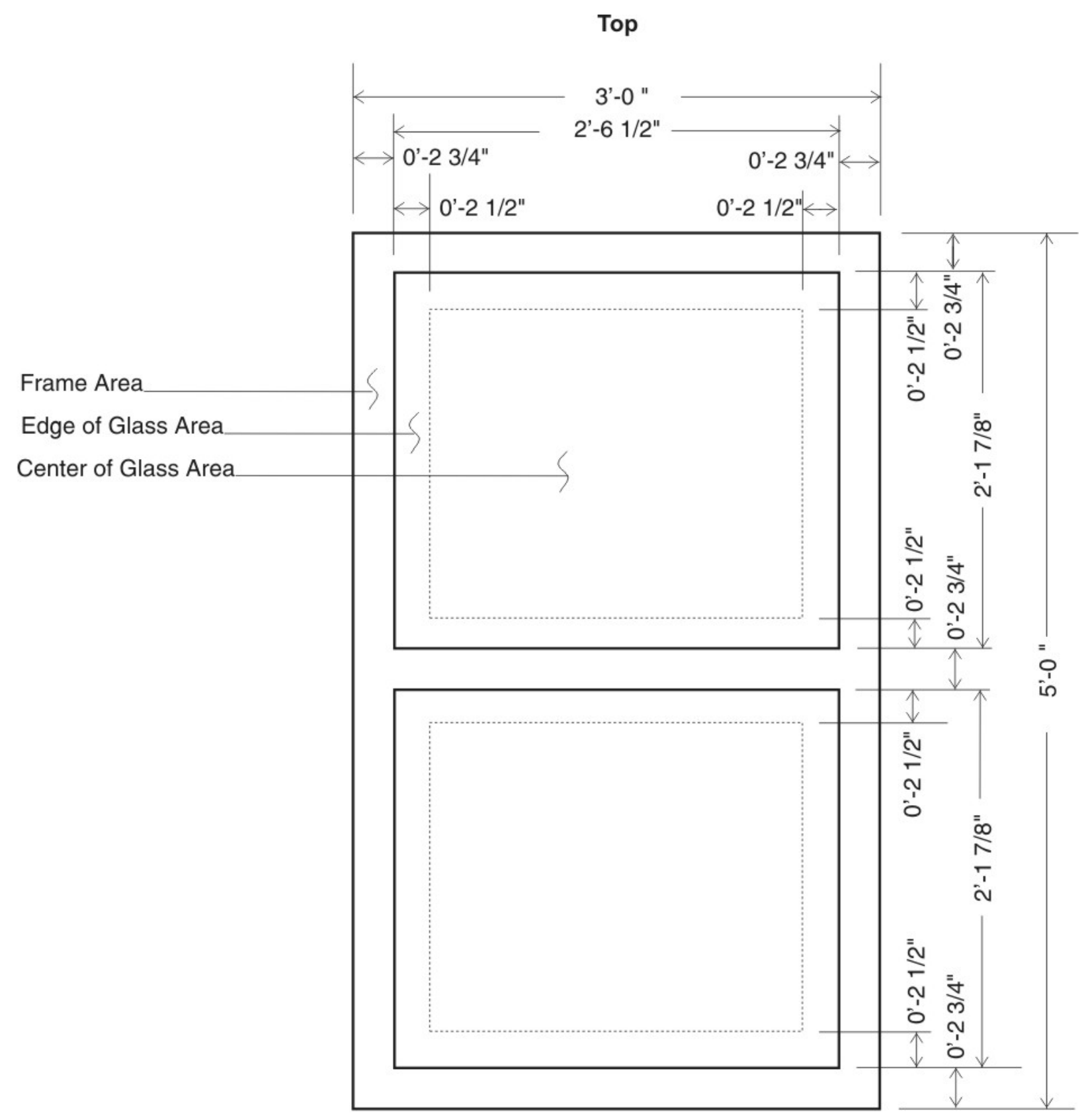

CD-RH06-A0327303

Figure 1-8. Window detail, vertical slider (NFRC AA) with $2 \frac{3}{4}{ }^{\prime \prime}$-wide frame - Case L200EX 
Table 1-1. Building Thermal Summary - Case L200EX

\begin{tabular}{|c|c|c|c|c|c|c|c|c|c|c|c|c|c|}
\hline \multirow[b]{3}{*}{\begin{tabular}{||l} 
ELEMENT \\
(Notes 1a, 1 b)
\end{tabular}} & \multirow[b]{3}{*}{$\begin{array}{c}\text { AREA } \\
\mathrm{ft}^{2}\end{array}$} & \multicolumn{3}{|c|}{ R-Value (Note 2a) } & \multicolumn{3}{|c|}{ U-Value (Note 2a) } & \multirow{2}{*}{\multicolumn{3}{|c|}{ UA }} & \multirow{2}{*}{\multicolumn{3}{|c|}{$\begin{array}{l}\text { HEATCAP } \\
\text { Nominal }\end{array}$}} \\
\hline & & \multirow{2}{*}{$\begin{array}{c}\text { Min } \\
\mathrm{h} \cdot \mathrm{ft}^{2} \cdot \mathrm{F} / \\
\mathrm{Btu}\end{array}$} & \multirow{2}{*}{$\begin{array}{c}\text { Nominal } \\
\mathrm{h} \cdot \mathrm{ft}^{2} \cdot \mathrm{F} / \\
\mathrm{Btu}\end{array}$} & \multirow{2}{*}{$\begin{array}{c}\text { Max } \\
\mathrm{h} \cdot \mathrm{ft}^{2} \cdot \mathrm{F} / \\
\text { Btu }\end{array}$} & \multirow{2}{*}{$\begin{array}{c}\text { Min } \\
\text { Btu/ } \\
\left(\mathbf{h} \cdot \mathrm{ft}^{2} \cdot \mathrm{F}\right)\end{array}$} & \multirow{2}{*}{$\begin{array}{c}\text { Nominal } \\
\text { Btu/ } \\
\left(\mathrm{h} \cdot \mathrm{ft}^{2} \cdot \mathrm{F}\right)\end{array}$} & \multirow{2}{*}{$\begin{array}{c}\text { Max } \\
\text { Btu/ } \\
\left(\mathbf{h} \cdot \mathrm{ft}^{2} \cdot \mathrm{F}\right)\end{array}$} & & & & & & \\
\hline & & & & & & & & $\begin{array}{c}\text { Min } \\
\text { Btu/(h.F) }\end{array}$ & $\begin{array}{l}\text { Nominal } \\
\text { Btu } /(\mathrm{h} \cdot \mathrm{F})\end{array}$ & $\underset{\text { Btu/(h.F })}{\operatorname{Max}}$ & $\begin{array}{c}\text { Min } \\
\text { Btu/F }\end{array}$ & $\begin{array}{c}\text { Btu/F } \\
\text { (Note } 2 b)\end{array}$ & $\begin{array}{l}\text { Max } \\
\text { Btu/F }\end{array}$ \\
\hline Exterior Walls (Note 3) & 1034 & 4.50 & 5.09 & 6.20 & 0.161 & 0.196 & 0.222 & 166.8 & 203.1 & 229.8 & & 1356 & \\
\hline North Windows (Note 4) & 90 & & 1.29 & & & 0.774 & & & 69.6 & & & & \\
\hline East Windows (Note 4) & 45 & & 1.29 & & & 0.774 & & & 34.8 & & & & \\
\hline West Windows (Note 4) & 45 & & 1.29 & & & 0.774 & & & 34.8 & & & & \\
\hline South Windows (Note 4) & 90 & & 1.29 & & & 0.774 & & & 69.6 & & & & \\
\hline Doors & 40 & & 3.28 & & & 0.305 & & & 12.2 & & & 62 & \\
\hline Ceiling/Attic/Roof (Notes 5a, 5b) & 1539 & 7.10 & 13.67 & 19.30 & 0.052 & 0.073 & 0.141 & 79.7 & 112.6 & 216.8 & 1005 & 1356 & 1655 \\
\hline $\begin{array}{l}\text { Floor (Note } 5 a) \\
\text { Infiltration (Note } 6)\end{array}$ & & & & & & & & & 76.8 & & & 1881 & \\
\hline Colorado Springs, CO & & & & & & & & 94.1 & 133.9 & 147.1 & & & \\
\hline Las Vegas, NV & & & & & & & & 70.5 & 100.6 & 110.4 & & & \\
\hline Interior Walls & 1024 & & & & & & & . & & & & 1425 & \\
\hline TOTAL BUILDING & & & & & & & & & & & 5729 & 6080 & 6379 \\
\hline Excluding Infiltration & & & & & & & & 544.4 & 613.5 & 744.4 & & & \\
\hline Including Infiltration (Colorado Sp & rings, $\mathrm{CO}$ ) & & & & & & & 638.5 & 747.4 & 891.6 & & & \\
\hline Including Infiltration (Las Vegas, & & & & & & & & 614.9 & 714.1 & 854.8 & & & \\
\hline \multicolumn{8}{|c|}{ WINDOW SUMMARY: SINGLE PANE, ALUMINUM FRAME WITH THERMAL BREAK } & \multirow{2}{*}{\multicolumn{3}{|c|}{$\begin{array}{l}\text { Trans. (Note 9) } \\
\text { (dir. nor.) }\end{array}$}} & \multirow{2}{*}{\multicolumn{3}{|c|}{$\begin{array}{c}\text { SC } \\
\text { (Note 9) }\end{array}$}} \\
\hline$($ Note 7$)$ & $\begin{array}{c}\text { Area } \\
\mathrm{ft}^{2}\end{array}$ & \multicolumn{3}{|c|}{$\begin{array}{c}\mathrm{U}(\text { Note } 2 \mathrm{a}) \\
\mathrm{Btu} /\left(\mathrm{h} \cdot \mathrm{ft}^{2} \cdot \mathrm{F}\right)\end{array}$} & \multicolumn{3}{|c|}{$\begin{array}{c}\text { SHGC (Note 8) } \\
\text { (dir. nor.) }\end{array}$} & & & & & & \\
\hline $\begin{array}{l}\text { Glass pane } \\
\text { Aluminum sash } w / \text { thermal break }\end{array}$ & $\begin{array}{r:r}10.96 \\
4.04\end{array}$ & & $\begin{array}{l}0.770 \\
0.785\end{array}$ & & & 0.862 & & \multicolumn{3}{|c|}{$\frac{\text { (dir. nor.) }}{0.837}$} & \multicolumn{3}{|c|}{0.991} \\
\hline Window, composite & 15.00 & & 0.774 & & & 0.679 & & & 0.612 & & & 0.780 & \\
\hline \multirow{2}{*}{\multicolumn{14}{|c|}{ 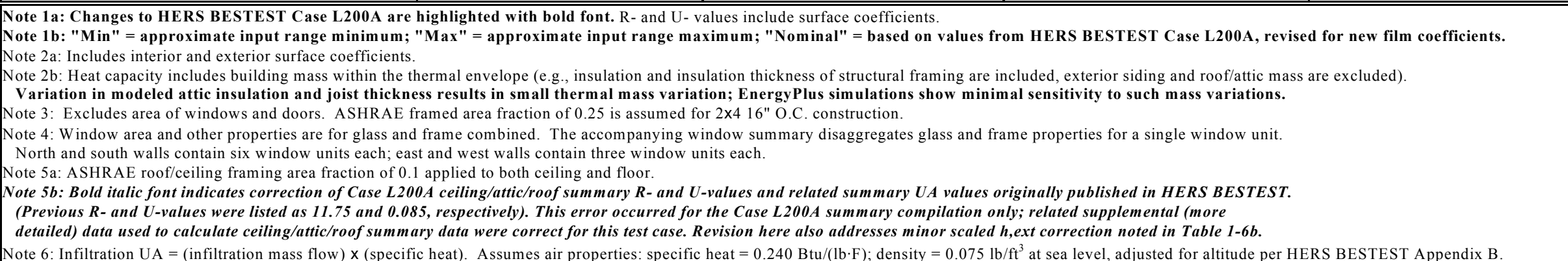 }} \\
\hline & & & & & & & & & & & & & \\
\hline \multirow{3}{*}{\multicolumn{2}{|c|}{$\begin{array}{l}\text { The following values were used to obtain infiltration UA } \\
\text { (see Tables } 1-8 \mathrm{a} \text { and } 1-8 \mathrm{~b} \text { for supporting details): }\end{array}$}} & & Location & ACH Min & ACH nominal & ACH Max & Volume $\left(\mathrm{ft}^{3}\right)$ & Altitude (ft) & Min & \multicolumn{2}{|c|}{$\operatorname{UAinf}(\mathrm{Btu} /(\mathrm{h} \cdot \mathrm{F}))$} & Max & \\
\hline & & & Colo Sprgs & 0.534 & 0.760 & $\mathbf{0 . 8 3 5}$ & 12312 & 6171 & 94.1 & 133.9 & & 147.1 & \\
\hline & & & Las Vegas & 0.345 & 0.492 & 0.540 & 12312 & 2178 & 70.5 & 100.6 & & 110.4 & \\
\hline
\end{tabular}


Table 1-2. Other Building Details - Case L200EX

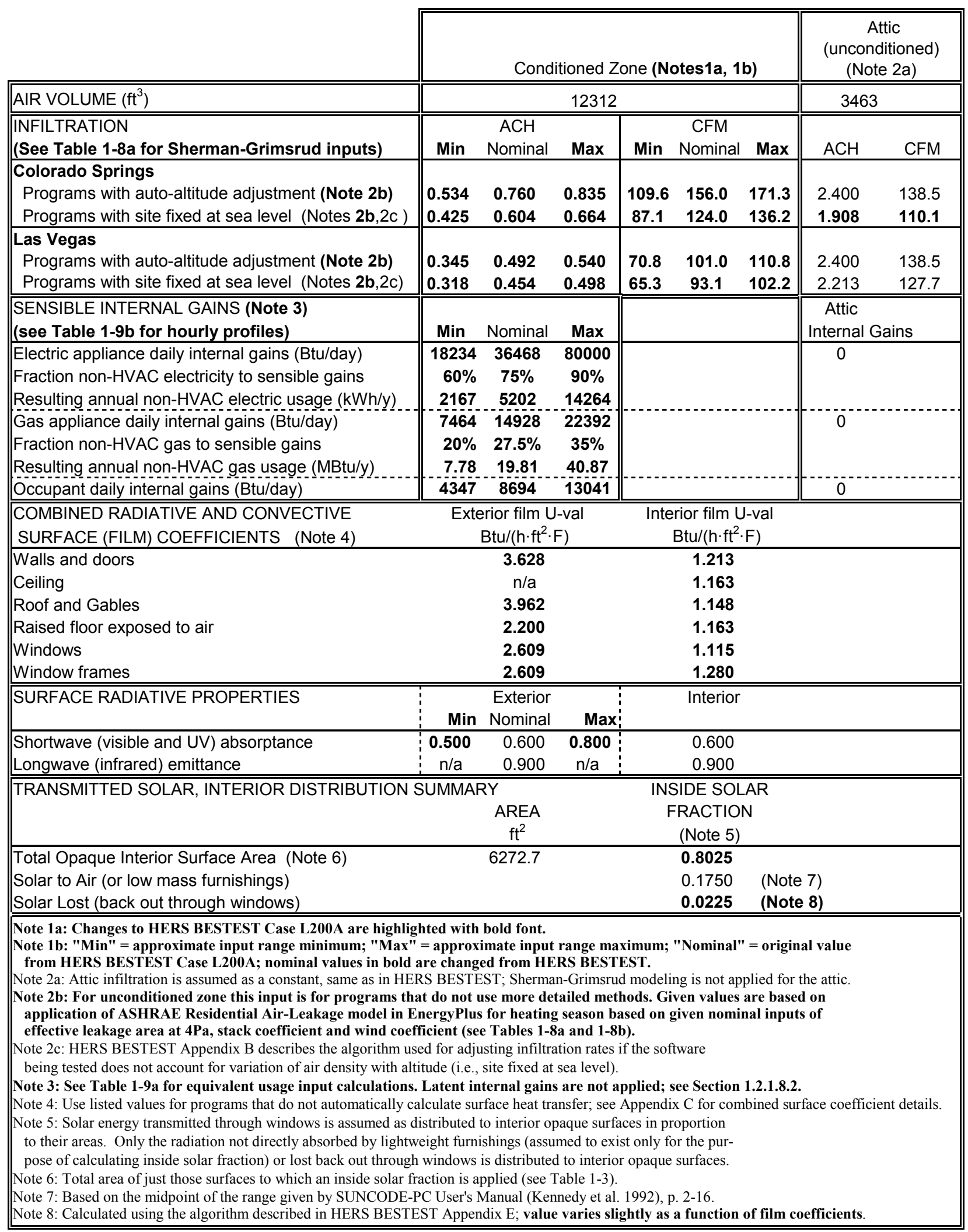


Table 1-3. Component Surface Areas and Solar Fractions - Case L200EX

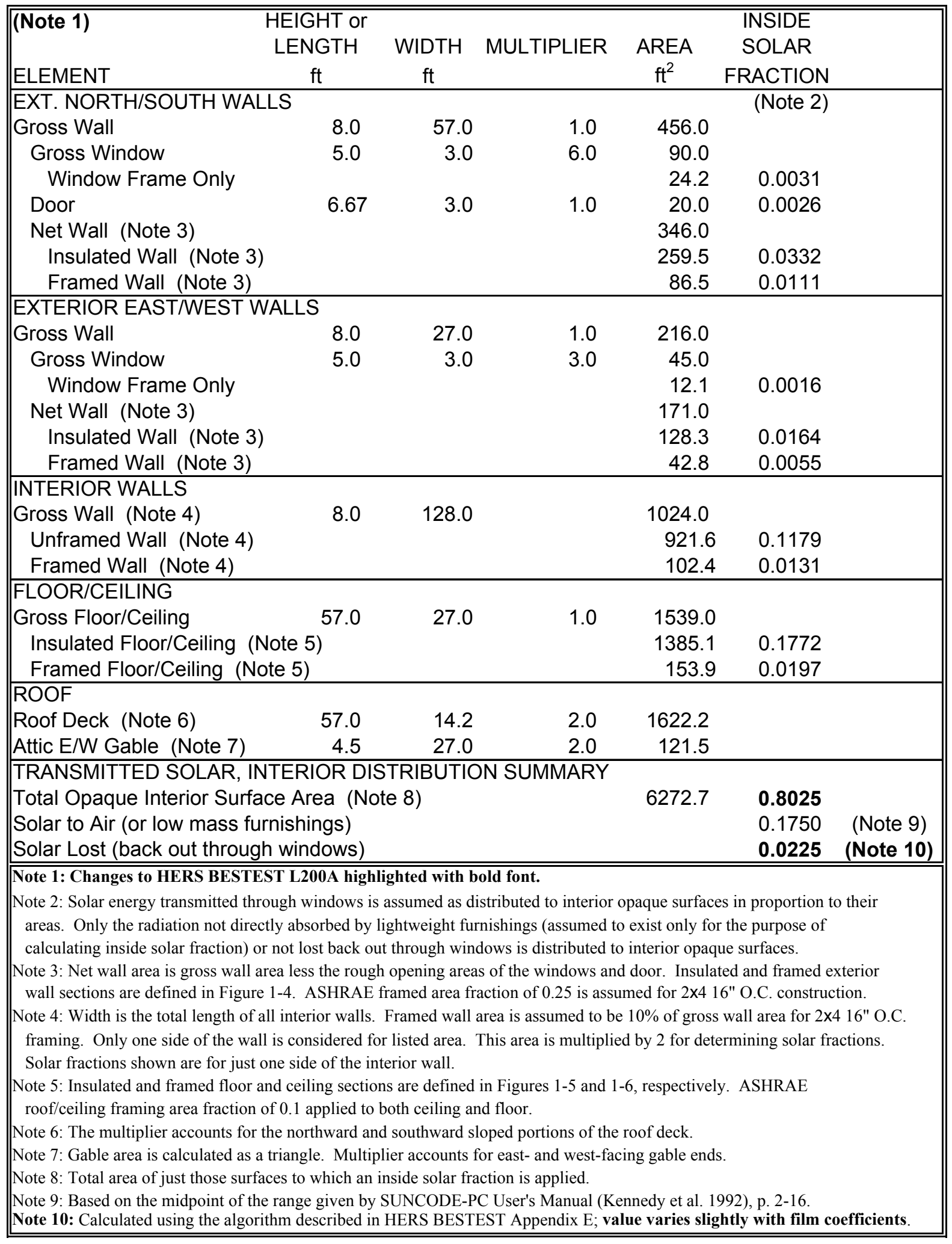


Table 1-4. Material Descriptions Exterior Wall, Door, and Window - Case L200EX

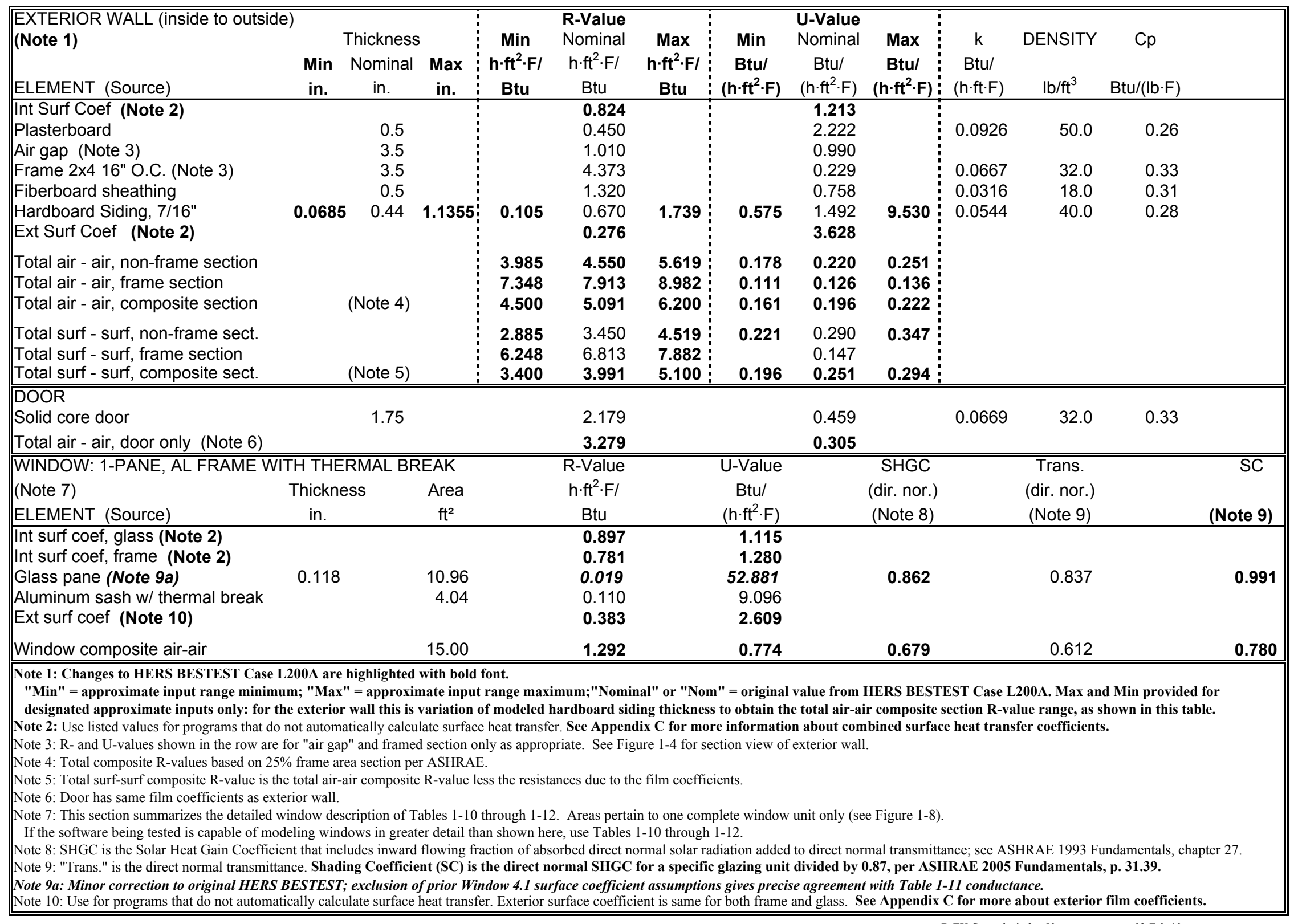

$$
\text { B-EX-Spec.xls i:a2..p50 02-Feb-10 }
$$


Table 1-5. Material Descriptions, Raised Floor Exposed to Air - Case L200EX

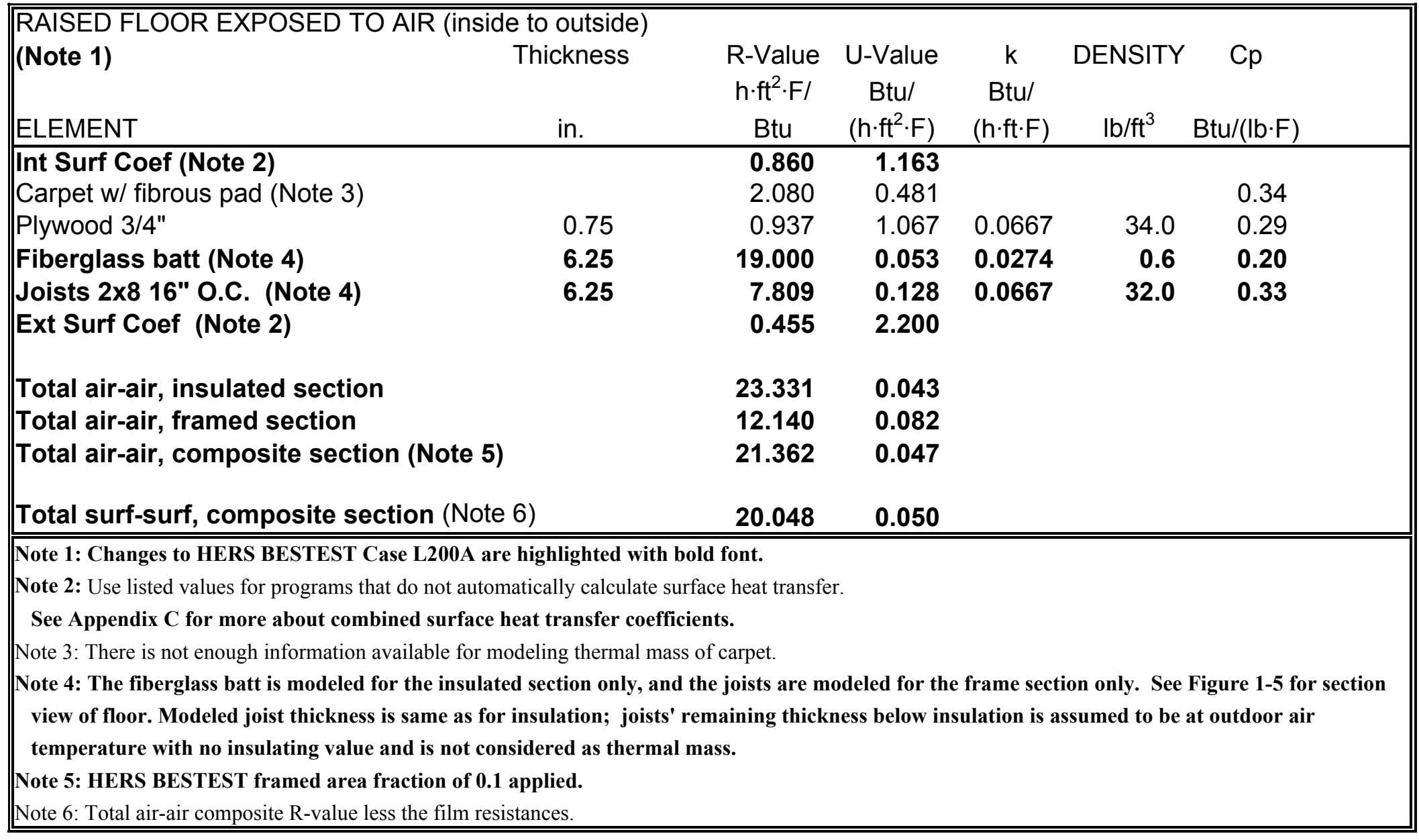


Table 1-6a. Material Descriptions, Ceiling, Attic, and Roof - Case L200EX

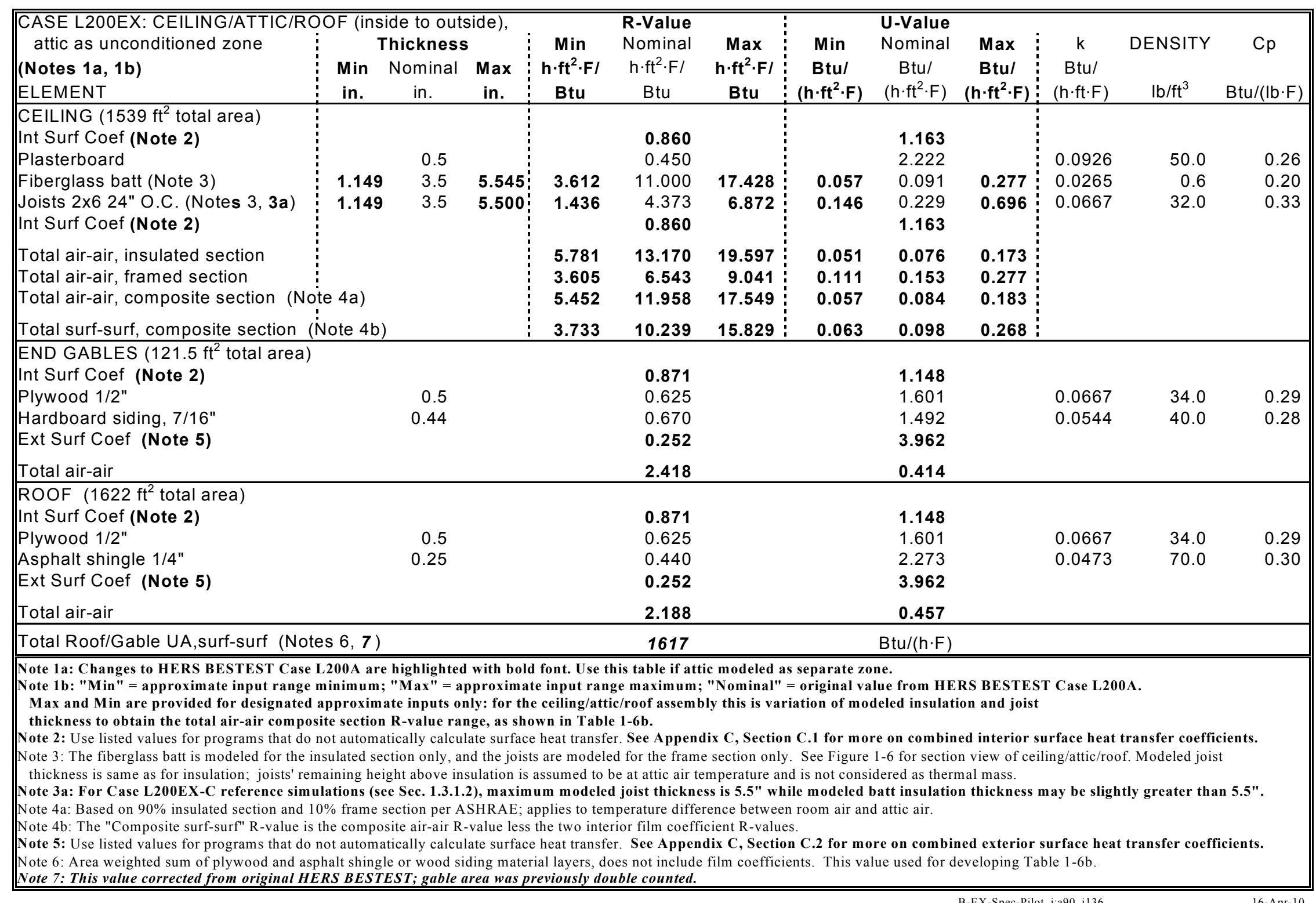


Table 1-6b. Material Descriptions, Ceiling/Attic/Roof, Attic as Material Layer - Case L200EX

\begin{tabular}{|c|c|c|c|c|c|c|c|c|c|c|c|c|}
\hline \multirow{2}{*}{\multicolumn{4}{|c|}{$\begin{array}{l}\text { CASE L200EX: CEILING/ATTIC/ROOF (inside to outside) } \\
\text { (Notes 1a, 1b) }\end{array}$}} & \multirow{3}{*}{$\begin{array}{c}\text { Min } \\
\mathrm{h} \cdot \mathrm{ft}^{2} \cdot \mathrm{F} / \\
\text { Btu }\end{array}$} & \multirow{3}{*}{$\begin{array}{c}\text { R-Value } \\
\text { Nominal } \\
\mathrm{h} \cdot \mathrm{ft}^{2} \cdot \mathrm{F} / \\
\mathrm{Btu} \\
\end{array}$} & \multirow{3}{*}{$\begin{array}{c}\text { Max } \\
\mathrm{h} \cdot \mathrm{ft}^{2} \cdot \mathrm{F} / \\
\text { Btu }\end{array}$} & \multirow{3}{*}{$\begin{array}{c}\text { Min } \\
\text { Btu/ } \\
\left(\mathbf{h} \cdot \mathrm{ft}^{2} \cdot \mathbf{F}\right)\end{array}$} & \multirow{3}{*}{$\begin{array}{c}\text { U-Value } \\
\text { Nominal } \\
\text { Btu/ } \\
\left(\mathrm{h} \cdot \mathrm{ft}^{2} \cdot \mathrm{F}\right)\end{array}$} & \multirow{3}{*}{$\begin{array}{c}\text { Max } \\
\text { Btul } \\
\left(\mathbf{h} \cdot \mathrm{ft}^{2} \cdot \mathrm{F}\right)\end{array}$} & \multirow{3}{*}{$\begin{array}{c}\mathbf{k} \\
\text { Btu/ } \\
(\mathrm{h} \cdot \mathrm{ft} \cdot \mathrm{F})\end{array}$} & \multirow{3}{*}{$\begin{array}{c}\text { DENSITY } \\
\mathrm{lb} / \mathrm{ft}^{3}\end{array}$} & \multirow{3}{*}{$\begin{array}{c}\mathrm{Cp} \\
\mathrm{Btu} /(\mathrm{lb} \cdot \mathrm{F})\end{array}$} \\
\hline & & & & & & & & & & & & \\
\hline & $\begin{array}{l}\text { Min } \\
\text { in. }\end{array}$ & $\begin{array}{l}\text { Nominal } \\
\text { in. }\end{array}$ & $\begin{array}{l}\text { Max } \\
\text { in. }\end{array}$ & & & & & & & & & \\
\hline \multicolumn{13}{|c|}{ CEILING/ATTIC AIR (1539 ft2 total area) } \\
\hline Int Surf Coef (Note 2) & & & & & & & & 1.163 & & & & \\
\hline Plasterboard & & 0.5 & & & 0.450 & & & 2.222 & & 0.0926 & 50.0 & 0.26 \\
\hline Fiberglass batt (Note 3) & 1.149 & 3.5 & 5.545 & 3.612 & 11.000 & 17.428 & 0.057 & 0.091 & 0.277 & 0.0265 & 0.6 & 0.20 \\
\hline Joists 2x6 24" O.C. (Notes 3, 3a) & 1.149 & 3.5 & 5.500 & 1.436 & 4.373 & 6.872 & 0.146 & 0.229 & 0.696 & 0.0667 & 32.0 & 0.33 \\
\hline Attic air (Note 4) & & & & & 1.300 & & & 0.769 & & & & \\
\hline \multicolumn{13}{|c|}{ RÖOF DECK AND GABLE PROPERTIES SCALED TO CEEILING AREA, $1539 \mathrm{ft}^{2}$ (Note $5 \mathrm{a}$ ) } \\
\hline Plywood 1/2" (Note 6) & & 0.5 & & & 0.551 & & & 1.814 & & 0.0756 & 38.5 & 0.29 \\
\hline Hybrid shingle/siding (Notes $5 b, 6)$ & & 0.25 & & & 0.400 & & & 2.499 & & 0.0521 & 79.3 & 0.30 \\
\hline \multicolumn{5}{|c|}{ Total roof deck/gable, surf-surf (Notes $5 c, 6$ ) } & 0.951 & & & 1.051 & & & & \\
\hline \multicolumn{5}{|l|}{ Ext Surf Coef (Note 2) } & 0.223 & & & 4.489 & & & & \\
\hline \multicolumn{13}{|l|}{ SUMMARY CEILING/ATTIC/ROOF } \\
\hline Total air-air, insulated section & (Note 7a) & & & 7.396 & 84 & 21.212 & 0.047 & 0.068 & 0.135 & & & \\
\hline Total air-air, framed section & (Note 7a) & & & 5.220 & 57 & 10.656 & 94 & 0.123 & 0.192 & & & \\
\hline Total air-air, composite section & (Note $7 b)$ & & & 7.100 & 13.673 & 19.300 & 0.052 & 0.073 & 0.141 & & & \\
\hline Total surf-surf, composite section & (Note 8) & & & 6.017 & 12.591 & 18.217 & 0.055 & 0.079 & 0.166 & & & \\
\hline \multicolumn{13}{|c|}{$\begin{array}{l}\text { Note 1a: Changes to HERS BESTEST Case L200A are highlighted with bold font. Use this table if attic modeled as material layer. } \\
\text { Note 1b: "Min" = approximate input range minimum; "Max" = approximate input range maximum; "Nominal" = original value from HERS BESTEST Case L200A. } \\
\text { Max and Min are only provided for designated approximate inputs: for the ceiling/attic/roof assembly this is variation of modeled insulation and joist } \\
\text { thickness to obtain the total air-air composite section R-value range, as shown. }\end{array}$} \\
\hline \multirow{2}{*}{\multicolumn{13}{|c|}{$\begin{array}{l}\text { Note 2: Use listed values for programs that do not automatically calculate surface heat transfer. See Appendix C for more on combined surface heat transfer coefficients. } \\
\text { Note 3: The fiberglass batt is modeled for the insulated section only, and the joists are modeled for the frame section only. See Figure 1-6 for section view of ceiling/attic/roof. Modeled joist thickness } \\
\text { is same as for insulation; joists' remaining height above insulation is assumed to be at attic air temperature and is not considered for thermal mass. }\end{array}$}} \\
\hline & & & & & & & & & & & & \\
\hline \multirow{2}{*}{\multicolumn{13}{|c|}{$\begin{array}{l}\text { Note 3a: For Case L200EX-C reference simulations (see Sec. 1.3.1.2), maximum modeled joist thickness is 5.5" while modeled batt insulation thickness may be slightly greater than 5.5". } \\
\text { Note 4: Average winter/summer values for natural ventilation }(2.4 \mathrm{ACH}), \mathrm{R}-11 \text { ceiling insulation, ext abs }=0.6 \text {., includes interior films. } \\
\text { Based on ASHRAE Load Calculation Manual, 1992, Tables } 4.5 \text { and 4.5a, "Effective Resistances of Ceiling or Attic Air Spaces." }\end{array}$}} \\
\hline & & & & & & & & & & & & \\
\hline \multirow{3}{*}{\multicolumn{13}{|c|}{$\begin{array}{l}\text { Note 5a: Scaled properties are presented for use with ASHRAE equivalent at } \\
\text { Note 5b: This "material" combines roofing and end gable materials into one h } \\
\text { Note 5c: Based on total roof/gable "UA,surf-surf" calculated in Table 1-6a. }\end{array}$}} \\
\hline & & & & & & & & & & & & \\
\hline & & & & & & & & & & & & \\
\hline \multicolumn{13}{|c|}{ Note 6: Minor errata note: Values corrected from original HERS BESTEST; gable area was previously double counted in scaling. Previous R-value for } \\
\hline \multicolumn{13}{|c|}{$\begin{array}{l}\text { "Total roof deck/gable, surf-surf" }=\mathbf{0 . 8 9 9} \text { hft }{ }^{2} \text { F/Btu would have resulted in } 0.3 \% \text { overall R-value effect versus original value. This correction is propagated through to summary Table 1-1. } \\
\text { Note 7a: (ceiling interior film coefficient) + (ceiling materials) }+ \text { (attic as material layer) }+(\text { scaled roof deck/gable materials) }+ \text { (scaled exterior film coefficient), for a given section. } \\
\text { Note 7b: Based on } 10 \% \text { frame area fraction per ASHRAE; applies to temperature difference between room air and ambient air. } \\
\text { Note 8: Based on total air-air composite R-value less R-values of interior film coefficient and scaled exterior film coefficient. }\end{array}$} \\
\hline
\end{tabular}


Table 1-8b. Equivalent Seasonal Constant Infiltration ACH and CFM - Case L200EX

\begin{tabular}{|c|c|c|c|c|c|c|c|c|}
\hline \multirow{3}{*}{\begin{tabular}{|l} 
AIR VOLUME $\left(\mathrm{ft}^{3}\right)$ \\
INFILTRATION
\end{tabular}} & \multicolumn{6}{|c|}{ Conditioned Zone (Notes1a, 1b) } & \multicolumn{2}{|c|}{$\begin{array}{c}\text { Attic } \\
\text { (unconditioned) } \\
\text { (Note 2) }\end{array}$} \\
\hline & \multicolumn{6}{|c|}{12312} & \multicolumn{2}{|c|}{3463} \\
\hline & Min & $\begin{array}{c}\mathrm{ACH} \\
\text { Nominal }\end{array}$ & Max & Min & $\begin{array}{c}\text { CFM } \\
\text { Nominal }\end{array}$ & Max & $\mathrm{ACH}$ & CFM \\
\hline \begin{tabular}{|l|} 
Colorado Springs \\
Programs with auto-altitude adjustment (Note 3) \\
Programs with site fixed at sea level (Notes 3,4) \\
\end{tabular} & $\begin{array}{l}0.534 \\
0.425 \\
\end{array}$ & $\begin{array}{l}0.760 \\
0.604 \\
\end{array}$ & $\begin{array}{l}0.835 \\
0.664 \\
\end{array}$ & $\begin{array}{c}109.6 \\
87.1 \\
\end{array}$ & $\begin{array}{l}156.0 \\
124.0 \\
\end{array}$ & $\begin{array}{l}171.3 \\
136.2 \\
\end{array}$ & $\begin{array}{l}2.400 \\
1.908 \\
\end{array}$ & $\begin{array}{l}138.5 \\
110.1 \\
\end{array}$ \\
\hline $\begin{array}{l}\text { Las Vegas } \\
\text { Programs with auto-altitude adjustment (Note 3) } \\
\text { Programs with site fixed at sea level (Notes } 3,4)\end{array}$ & $\begin{array}{l}0.345 \\
0.318\end{array}$ & $\begin{array}{l}0.492 \\
0.454\end{array}$ & $\begin{array}{l}0.540 \\
0.498\end{array}$ & $\begin{array}{l}70.8 \\
65.3\end{array}$ & $\begin{array}{c}101.0 \\
93.1\end{array}$ & $\begin{array}{l}110.8 \\
102.2\end{array}$ & $\begin{array}{l}2.400 \\
2.213\end{array}$ & $\begin{array}{l}138.5 \\
127.7\end{array}$ \\
\hline $\begin{array}{l}\text { Note 1a: Changes to HERS BESTEST Case L200A are hig } \\
\text { Note 1b: "Min" = approximate input range minimum; "M } \\
\text { from HERS BESTEST Case L200A; nominal values in b } \\
\text { Note 2: Attic infiltration is assumed as a constant, same as in } \\
\text { Note 3: For conditioned zone this input is for programs th } \\
\text { application of ASHRAE Residential Air-Leakage model } \\
\text { (e.g. October - April for heating) as appropriate, based o } \\
\text { and wind coefficient (see Table 1-8a). See Appendix D fo } \\
\text { Note 4: HERS BESTEST Appendix B describes the algorithm } \\
\text { being tested does not account for variation of air density wit }\end{array}$ & $\begin{array}{l}\text { lighted } \mathbf{w} \\
\mathrm{x}^{\prime \prime}=\text { app } \\
\text { d are ch: } \\
\text { ERS BES } \\
\text { do not u } \\
\text { Energyl } \\
\text { given no } \\
\text { supporti } \\
\text { ised for a } \\
\text { altitude ( }\end{array}$ & $\begin{array}{l}\text { th bold for } \\
\text { oximate in } \\
\text { nged from } \\
\text { EST; Sher } \\
\text { e more det } \\
\text { lus for the } \\
\text { ninal inpu } \\
\text { g detail. } \\
\text { justing infil } \\
\text { e., site fixe }\end{array}$ & $\begin{array}{l}\text { ut range } \\
\text { IERS BE } \\
\text { an-Grims } \\
\text { iled meth } \\
\text { eating or } \\
\text { of effect } \\
\text { ation rate } \\
\text { at sea lev }\end{array}$ & $\begin{array}{l}\text { Iaximum } \\
\text { TEST. } \\
\text { Id modeli } \\
\text { ds. Giver } \\
\text { ooling se } \\
\text { e leakage } \\
\text { if the sof } \\
\text { ). }\end{array}$ & $\begin{array}{l}\text { "Nominal" } \\
\mathrm{g} \text { is not appl } \\
\text { values are a } \\
\text { son rounde } \\
\text { area at } 4 \mathrm{~Pa} \\
\text { vare }\end{array}$ & $\begin{array}{l}=\text { origina } \\
\text { ed for the } \\
\text { verages } \\
\text { d to the } n \\
\text { stack co }\end{array}$ & $\begin{array}{l}\text { value } \\
\text { attic. } \\
\text { sed on } \\
\text { arest mon } \\
\text { fficient, }\end{array}$ & \\
\hline
\end{tabular}

Table 1-9a. Daily Sensible Internal Loads - Case L200EX

\begin{tabular}{|c|c|c|c|}
\hline (Notes 1, 2, 3, 4, 5, 6) & $\begin{array}{c}\text { Occupants } \\
\text { Sensible } \\
\end{array}$ & $\begin{array}{c}\text { Electricity } \\
\text { Sensible }\end{array}$ & $\begin{array}{c}\text { Gas } \\
\text { Sensible }\end{array}$ \\
\hline \multicolumn{4}{|l|}{ Daily Sensible Load } \\
\hline Min (Btu/day) & 4,347 & 18,234 & 7,464 \\
\hline Nominal (Btu/day) & 8,694 & 36,468 & 14,928 \\
\hline Max (Btu/day) & 13,041 & 80,000 & 22,392 \\
\hline \multicolumn{4}{|l|}{ Internal Gains Fractions (Note 7) } \\
\hline Min - Max & $\mathrm{n} / \mathrm{a}$ & $60 \%-90 \%$ & $20 \%-35 \%$ \\
\hline Nominal & $\mathrm{n} / \mathrm{a}$ & $75 \%$ & $27.5 \%$ \\
\hline $\begin{array}{l}\text { Resulting Metered Annual non-HVAC } \\
\text { Energy Usage }\end{array}$ & & $\begin{array}{l}\text { Electricity } \\
\text { (kWh) }\end{array}$ & $\begin{array}{c}\text { Gas } \\
\text { (million Btu/yr) }\end{array}$ \\
\hline Min (Note 8) & $\mathrm{n} / \mathrm{a}$ & 2,617 & 7.78 \\
\hline Nominal (Note 9) & $\mathrm{n} / \mathrm{a}$ & 5,202 & 19.81 \\
\hline Max (Note 10) & $\mathrm{n} / \mathrm{a}$ & 14,264 & 40.87 \\
\hline $\begin{array}{l}\text { Note 1: All internal gains data are new (rela } \\
\text { Note 2: "Min" = approximate input range minim } \\
\text { range determination developed in Appendix B. } \\
\text { Note 3: For reference simulations hourly loads } \\
\text { their respective normalized hourly profiles in T } \\
\text { Note 4: Sensible gains are } 70 \% \text { radiative and } \\
\text { Note 5: The MAX value for "Electricity Sensible } \\
\text { Working Group (2009). } \\
\text { Note 6: Gas sensible loads are from DHW onl) } \\
\text { Note 7: Percentage of metered energy use tha } \\
\text { Note 8: = (Minimum Load/day) / (Maximum Int } \\
\text { Note 9: = (Nominal Load/day) / (Nominal Interr } \\
\text { Note 10: = (Maximum Load/day) / (Minimum Ir }\end{array}$ & $\begin{array}{l}\text { BESTEST) for } \\
\text { approximate inf } \\
\text { multiplying the } \\
\text { ve. } \\
\text { ed to } 80 \mathrm{kBtu} \text { ac } \\
\text { sible loads are } \\
\text { ensible internal } \\
\text { raction) }{ }^{*}(365 \\
\text { ction })^{*}(365 \text { da } \\
\text { Fraction }) *(365\end{array}$ & $\begin{array}{l}\text { st specificatic } \\
\text { e maximum; "I } \\
\text { aly selected da } \\
\text { g to consultatio } \\
\text { other non-HV/ } \\
\text { / (1000000 (/M } \\
1000000(/ \mathrm{M})) \\
/(1000000(/ /\end{array}$ & $\begin{array}{l}\text { is the basis value for } \\
\text { for each category by } \\
\text { BESTEST-EX } \\
\text { ances. } \\
\text { Btu] } \\
\text { tu] } \mathrm{Btu}]\end{array}$ \\
\hline
\end{tabular}


Table 1-9b. Normalized Hourly Profiles for Sensible Internal Loads - Case L200EX

\begin{tabular}{|c|c|c|c||}
\hline Hour (Note 1) & $\begin{array}{c}\text { Occupants } \\
\text { Sensible }\end{array}$ & $\begin{array}{c}\text { Electricity } \\
\text { Sensible }\end{array}$ & Gas Sensible \\
\hline 1 & 0.061 & 0.022 & 0.036 \\
2 & 0.061 & 0.020 & 0.035 \\
3 & 0.061 & 0.019 & 0.035 \\
4 & 0.061 & 0.020 & 0.035 \\
5 & 0.061 & 0.025 & 0.036 \\
6 & 0.061 & 0.038 & 0.039 \\
7 & 0.061 & 0.048 & 0.046 \\
8 & 0.052 & 0.050 & 0.048 \\
9 & 0.024 & 0.038 & 0.048 \\
10 & 0.015 & 0.034 & 0.047 \\
11 & 0.015 & 0.032 & 0.045 \\
12 & 0.015 & 0.031 & 0.044 \\
13 & 0.015 & 0.030 & 0.043 \\
14 & 0.015 & 0.029 & 0.042 \\
15 & 0.015 & 0.029 & 0.041 \\
16 & 0.015 & 0.034 & 0.041 \\
18 & 0.018 & 0.051 & 0.042 \\
19 & 0.032 & 0.065 & 0.043 \\
21 & 0.053 & 0.077 & 0.044 \\
22 & 0.053 & 0.083 & 0.044 \\
23 & 0.053 & 0.082 & 0.044 \\
Nate All internal gains data are new (relative to HERS BESTEST) for & & 0.043 \\
this test specification. & 0.061 & 0.066 & 0.041 \\
\hline \hline
\end{tabular}


Table 1-10. Window Summary (Single-Pane Aluminum Frame With Thermal Breaks) - Case L200EX

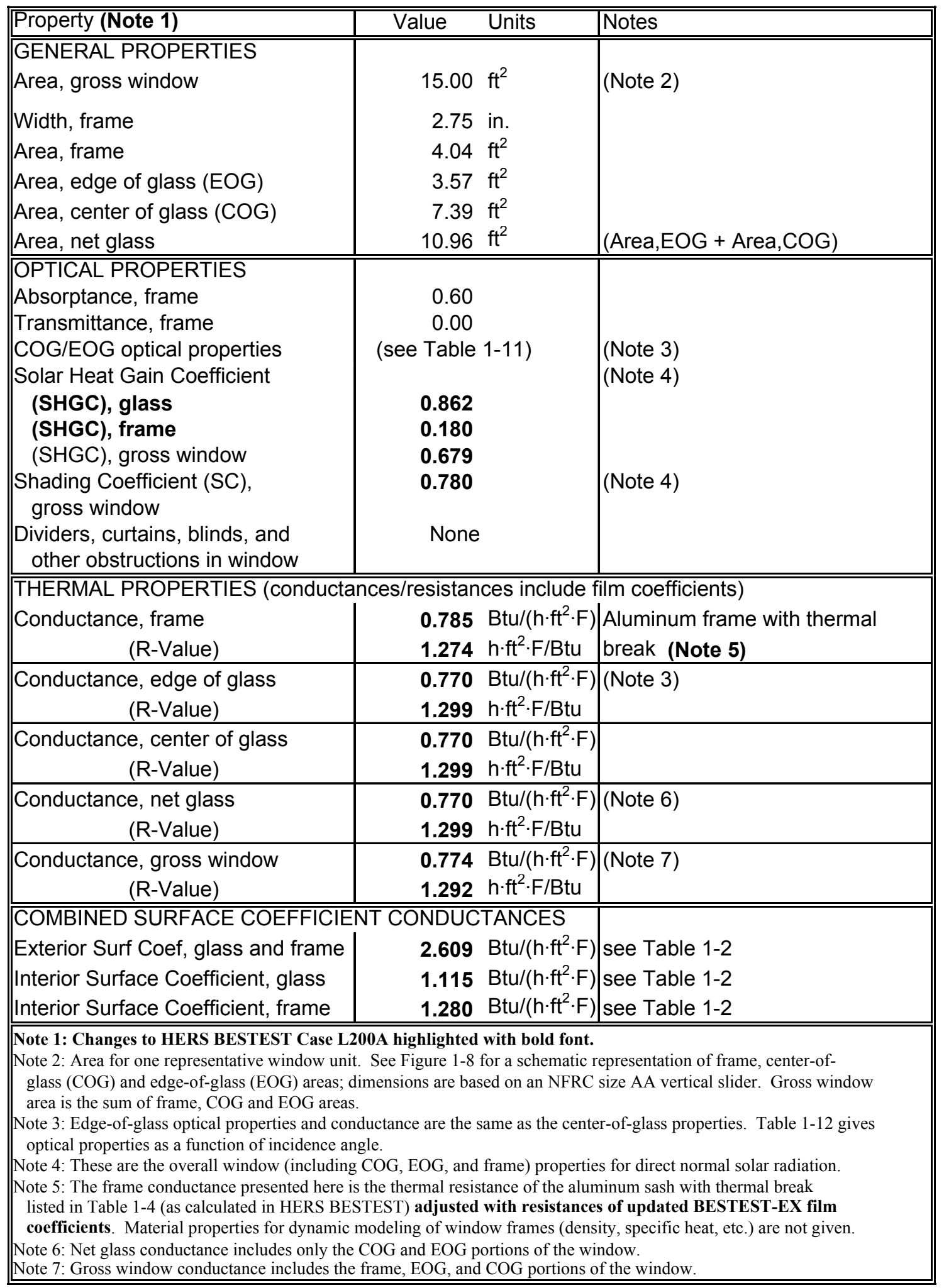


Table 1-11. Glazing Summary, Single-Pane Center of Glass Values - Case L200EX

\begin{tabular}{|c|c|c|}
\hline Property & Value & Notes \\
\hline $\begin{array}{l}\text { GENERAL PROPERTIES } \\
\text { Number of Panes } \\
\text { Pane Thickness }\end{array}$ & $\begin{array}{r}1 \\
0.118 \text { in. }\end{array}$ & (Note 1) \\
\hline $\begin{array}{l}\text { SINGLE PANE OPTICAL PROP. } \\
\text { Transmittance } \\
\text { Reflectance } \\
\text { Absorptance } \\
\text { Index of Refraction } \\
\text { Extinction Coefficient } \\
\text { Solar Heat Gain Coefficient (SHGC) } \\
\text { Shading Coefficient (SC) } \\
\text { Optical Properties as Function of Incident Angle }\end{array}$ & $\begin{array}{c}0.837 \\
0.075 \\
0.089 \\
1.5223 \\
0.7806 \text { lin. } \\
\mathbf{0 . 8 6 2} \\
\mathbf{0 . 9 9 1} \\
\text { (See Table 1-12) }\end{array}$ & (Note 2) \\
\hline $\begin{array}{l}\text { THERMAL PROPERTIES } \\
\text { Conductivity of Glass }\end{array}$ & $0.520 \mathrm{Btu} /(\mathrm{h} \cdot \mathrm{ft} \cdot \mathrm{F})$ & \\
\hline $\begin{array}{c}\text { Conductance of Glass Pane } \\
\text { (R-Value) }\end{array}$ & $\begin{array}{rl}52.881 & \mathrm{Btu} /\left(\mathrm{h} \cdot \mathrm{ft}^{2} \cdot \mathrm{F}\right) \\
0.019 & \mathrm{~h} \cdot \mathrm{ft}^{2} \cdot \mathrm{F} / \mathrm{Btu}\end{array}$ & \\
\hline $\begin{array}{c}\text { Exterior Combined Surface Coefficient } \\
\text { (R-Value) }\end{array}$ & $\begin{array}{ll}2.609 & \mathrm{Btu} /\left(\mathrm{h} \cdot \mathrm{ft}^{2} \cdot \mathrm{F}\right) \\
0.383 & \mathrm{~h} \cdot \mathrm{ft}^{2} \cdot \mathrm{F} / \mathrm{Btu}\end{array}$ & (Note 4) \\
\hline $\begin{array}{c}\text { Interior Combined Surface Coefficient } \\
\text { (R-Value) }\end{array}$ & $\begin{array}{ll}1.115 & \mathrm{Btu} /\left(\mathrm{h} \cdot \mathrm{ft}^{2} \cdot \mathrm{F}\right) \\
0.897 & \mathrm{~h} \cdot \mathrm{ft}^{2} \cdot \mathrm{F} / \mathrm{Btu}\end{array}$ & (Note 4) \\
\hline $\begin{array}{c}\text { U-Value from Interior Air to Ambient Air } \\
\text { (R-Value) }\end{array}$ & $\begin{array}{ll}0.770 & \mathrm{Btu} /\left(\mathrm{h} \cdot \mathrm{ft}^{2} \cdot \mathrm{F}\right) \\
1.299 & \mathrm{~h} \cdot \mathrm{ft}^{2} \cdot \mathrm{F} / \mathrm{Btu}\end{array}$ & \\
\hline $\begin{array}{l}\text { Hemispherical Infrared Emittance } \\
\text { Infrared Transmittance } \\
\text { Density of Glass } \\
\text { Specific Heat of Glass }\end{array}$ & $\begin{array}{rl}0.84 & \\
0 & \\
154 & \mathrm{lb} / \mathrm{ft}^{3} \\
0.18 & \mathrm{Btu} /(\mathrm{lb} \cdot \mathrm{F}) \\
\end{array}$ & \\
\hline \multicolumn{3}{|c|}{$\begin{array}{l}\text { Note 1: Changes to HERS BESTEST Case L200A highlighted with bold font. } \\
\text { Note 2: Optical properties listed in this table are for direct normal radiation. } \\
\text { Note 3: SC = SHGC/0.87 per Equation 91 (p. 31.39) of ASHRAE } 2005 \text { Fundamentals. } \\
\text { Note 4: See Table 1-2. }\end{array}$} \\
\hline
\end{tabular}


Table 1-12. Optical Properties as a Function of Incidence Angle for Single-Pane Glazing - Case L200EX

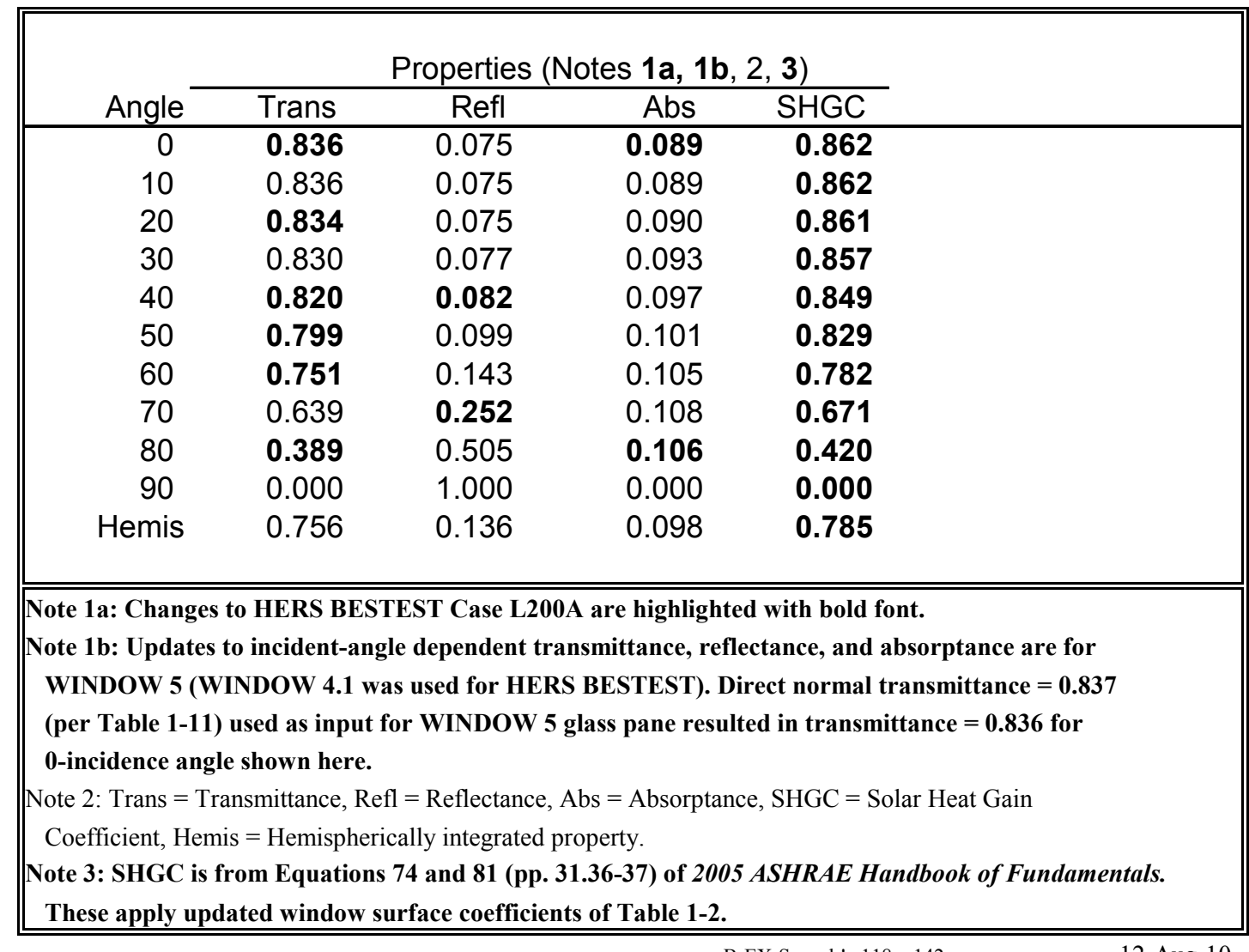

B-EX-Spec, b!:a118..g142

12-Aug-10

\subsubsection{Building Physics Retrofit Test Cases}

This section describes revisions to the base building required to model the retrofit test cases. For convenience, relevant portions of the appropriate base building tables and figures have been reprinted, with changes to the base-case model to be applied in the retrofit cases highlighted in bold font. Where applicable, summary figures and tables are listed first, with supplementary tables listed afterward.

\subsubsection{Case L210EX-P: Air-Seal Retrofit}

Case L210EX-P is exactly as Case L200EX-P, except for the following changes.

\subsection{Conditioned Zone}

Changes to detailed inputs for programs that apply Sherman-Grimsrud infiltration modeling are provided in Table 1-13. Use only the inputs that apply to the software being tested. For programs that do not apply Sherman-Grimsrud modeling, values for "equivalent seasonal constant ACH" (or CFM) are included in Table 1-14. The equivalence of the inputs is discussed in Section 1.2.1.7 and Appendix D.

If the program being tested applies a constant infiltration rate only and does not use barometric pressure from the weather data, or otherwise does not automatically correct for the change in air density caused by altitude, adjust the constant specified infiltration rates (to yield mass flows equivalent to what would occur at the specified altitude), as shown in Table 1-14. 


\subsection{Attic}

Attic infiltration is the same as in the base case (L200EX-P).

Table 1-13. Conditioned Zone Equivalent Inputs for Weather-Driven Infiltration Models Case L210EX-P

\begin{tabular}{|c|c|}
\hline Input (Note 1) & Value \\
\hline CFM at $50 \mathrm{~Pa}$ (Colorado Springs) (Note 2) & 2000 \\
\hline CFM at $50 \mathrm{~Pa}$ (Las Vegas) (Note 2) & 1857 \\
\hline Air Volume $\left(\mathrm{ft}^{3}\right)$ & 12312 \\
\hline $\mathrm{ACCH}$ at 50 Pa (Colorado Springs) (Note 2) & 9.75 \\
\hline $\mathrm{ACH}$ at $50 \mathrm{~Pa}$ (Las Vegas) (Note 2) & 9.05 \\
\hline Ältitude (Colorado Springs), ft & 6171 \\
\hline Altitude (Las Vegas), $\mathrm{ft}$ & 2178 \\
\hline Air density (Colorado Springs), İb/ft ${ }^{3}$ (Note 3) & 0.060 \\
\hline Air density (Las Vegas), lb/ft ${ }^{3}$ (Note 3 ) & 0.069 \\
\hline Equivalent Leakage Area at $50 \mathrm{~Pa}, \mathrm{in}^{2}$ (Note 4) & 143.3 \\
\hline Effective Leakage Area at $4 \mathrm{~Pa}$, in $^{2}$ (Note 4) & 98.1 \\
\hline Number of Building Stories & 1.0 \\
\hline ASHRAE Stack coefficient $\mathrm{cfm}^{2} /\left(\mathrm{in}^{4}{ }^{\circ} \mathrm{F}\right)$ (Note 4) & 0.0150 \\
\hline ASHRAE Wind coefficient $\mathrm{cfm}^{2} /\left(\mathrm{in}^{4} \mathrm{mph}^{2}\right)$ (Note 4) & 0.0012 \\
\hline \multicolumn{2}{|c|}{$\begin{array}{l}\text { Note 1: Changes to Case L200EX are highlighted with bold font. } \\
\text { Note 2: Volumetric flow rates are different for each climate. } \\
\text { Note 3: Calculation of air density as a function of altitude is described in HERS BESTEST, Appendix B. } \\
\text { Note 4: Used for ASHRAE Residential Air Leakage model (2005 ASHRAE Handbook of Fundamentals, pp. 27.12-27.13, 27.21); model is based on } \\
\text { Sherman-Grimsrud (1980), assuming highly sheltered building (Shelter Class 5) in rural terrain. See Appendix D for supporting details. }\end{array}$} \\
\hline
\end{tabular}

Table 1-14. Equivalent Seasonal Constant Infiltration ACH and CFM - Case L210EX-P

\begin{tabular}{|c|c|c|c|c|c|}
\hline & \multicolumn{3}{|c|}{ Conditioned Zone (Note 1) } & \multicolumn{2}{|c|}{ Attic (uncond.) } \\
\hline AIR VOLUME $\left(\mathrm{ft}^{3}\right)$ & \multicolumn{3}{|c|}{12312} & \multicolumn{2}{|c|}{3463} \\
\hline \multirow[t]{2}{*}{ INFILTRATION } & \multirow{2}{*}{\multicolumn{3}{|c|}{$\begin{array}{c}\text { UAinfl } \\
\text { Btu/(h.F) }\end{array}$}} & \multicolumn{2}{|c|}{ (Note 2) } \\
\hline & & & & $\mathrm{ACH}$ & CFM \\
\hline $\begin{array}{l}\text { Colorado Springs } \\
\text { Programs with auto-altitude adjustment (Note 3) } \\
\text { Programs with site fixed at sea level (Notes 3, 5) }\end{array}$ & $\begin{array}{l}0.382 \\
0.304\end{array}$ & $\begin{array}{l}78.4 \\
62.3\end{array}$ & 67.3 (Note 4) & $\begin{array}{l}2.400 \\
1.908\end{array}$ & $\begin{array}{l}138.5 \\
110.1\end{array}$ \\
\hline $\begin{array}{l}\text { Las Vegas } \\
\text { Programs with auto-altitude adjustment (Note 3) } \\
\text { Programs with site fixed at sea level (Notes 3, 5) }\end{array}$ & $\begin{array}{l}0.246 \\
0.227 \\
\end{array}$ & $\begin{array}{l}50.5 \\
46.6 \\
\end{array}$ & 50.3 (Note 4) & $\begin{array}{l}2.400 \\
2.213 \\
\end{array}$ & $\begin{array}{l}138.5 \\
127.7 \\
\end{array}$ \\
\hline $\begin{array}{l}\text { Note 1: Changes to Case L200EX are highlighted with bol } \\
\text { Note 2: Attic infiltration is same as Case L200EX; Sherman-G } \\
\text { Note 3: For conditioned zone this input is for programs that do } \\
\text { application of ASHRAE Residential Air-Leakage model in E } \\
\text { given inputs of effective leakage area at 4Pa, stack coefficier } \\
\text { Note 4: Infiltration UA = (infiltration mass flow) } \text { ( specific he } \\
\text { sea level, adjusted for altitude per HERS BESTEST Append } \\
\text { Note 5: HERS BESTEST Appendix B describes the algorithm } \\
\text { variation of air density with altitude (i.e., site fixed at sea lev }\end{array}$ & $\begin{array}{l}\text { eling is } n \\
\text { e detaile } \\
r \text { approp } \\
\text { coefficien } \\
\text { s air pro } \\
\text { usting inf }\end{array}$ & $\begin{array}{l}\text { applied } \\
\text { lethods. } \\
\text { e space } \\
\text { see Tab } \\
\text { ties: spe } \\
\text { ation rat }\end{array}$ & $\begin{array}{l}\text { r the attic. } \\
\text { iven values are average } \\
\text { onditioning season basec } \\
\text { 1-13). } \\
\text { fic heat }=0.240 \mathrm{Btu} /(\mathrm{lb} \\
\mathrm{s} \text { if the software being te }\end{array}$ & $=0.0751$ & $\mathrm{ft}^{3}$ at \\
\hline
\end{tabular}




\subsubsection{Case L220EX-P: Attic Insulation Retrofit}

Case L220EX-P is exactly as Case L200EX-P, except that 2.0 in. of cellulose is blown between the joists on top of the existing fiberglass batt with a further $6.0 \mathrm{in.} \mathrm{of} \mathrm{blown} \mathrm{cellulose} \mathrm{covering} \mathrm{the} \mathrm{joists} \mathrm{and}$ cellulose (see Figure 1-9). The full joist thickness (5.5 in.) is modeled in this case. Use inputs from Case L200EX-P, except for changes called out by the retrofit (highlighted with bold font in figures and tables cited below). The following figure and tables highlight the retrofit.

Figure 1-9

Ceiling section - Case L220EX

Table 1-15

Building Thermal Summary - Case L220EX

Table 1-16a

Material Descriptions, Ceiling - Case L220EX

Table 1-16b

Material Descriptions for Attic as Material Layer - Case L220EX (for calculation of equivalent ceiling/attic/roof composite R-value see discussion of the base building attic in Section 1.2.1.4).

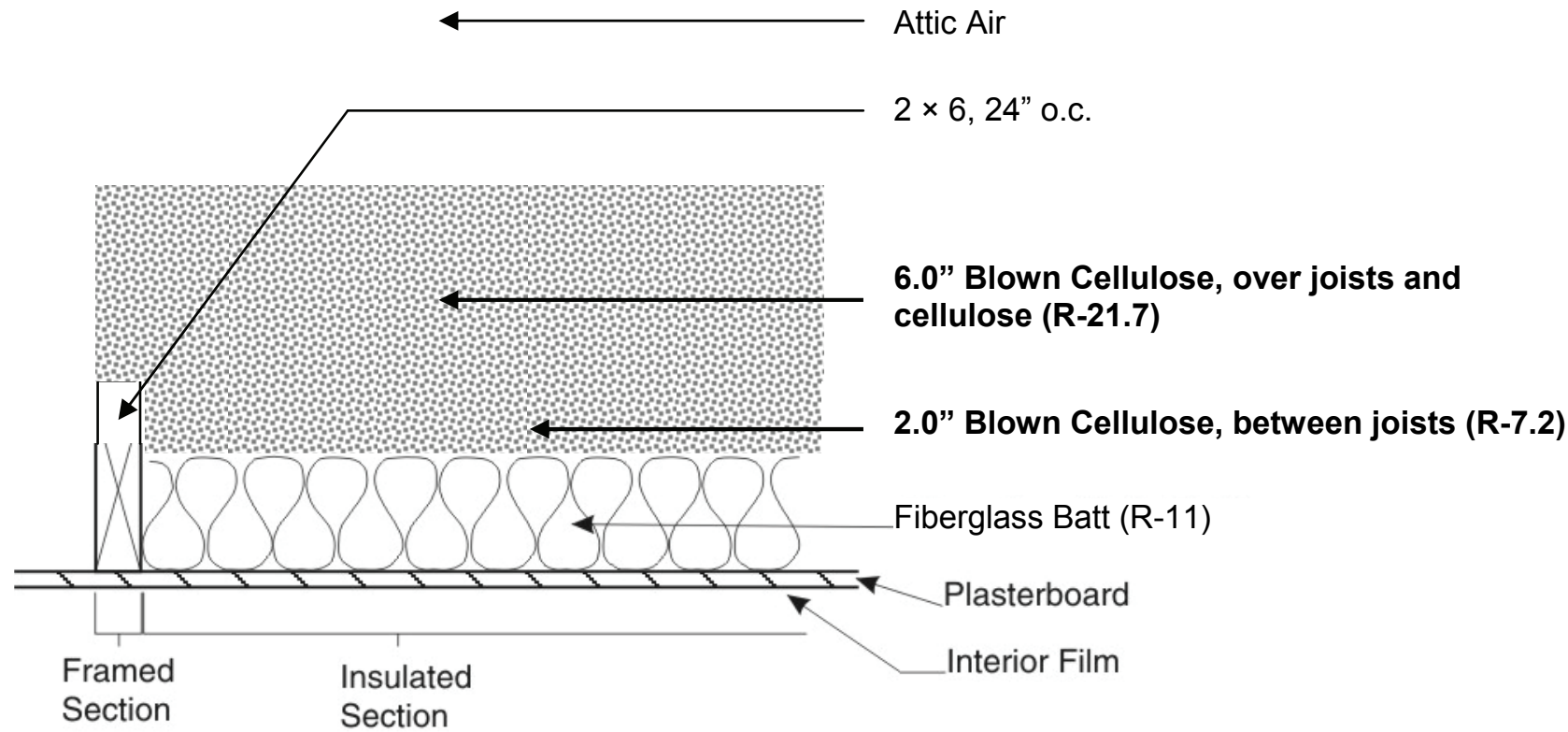

CD-RH06-A0327322

Note: Changes to Case L200EX are highlighted with bold font.

Figure 1-9. Ceiling section - Case L220EX 
Table 1-15. Building Thermal Summary - Case L220EX

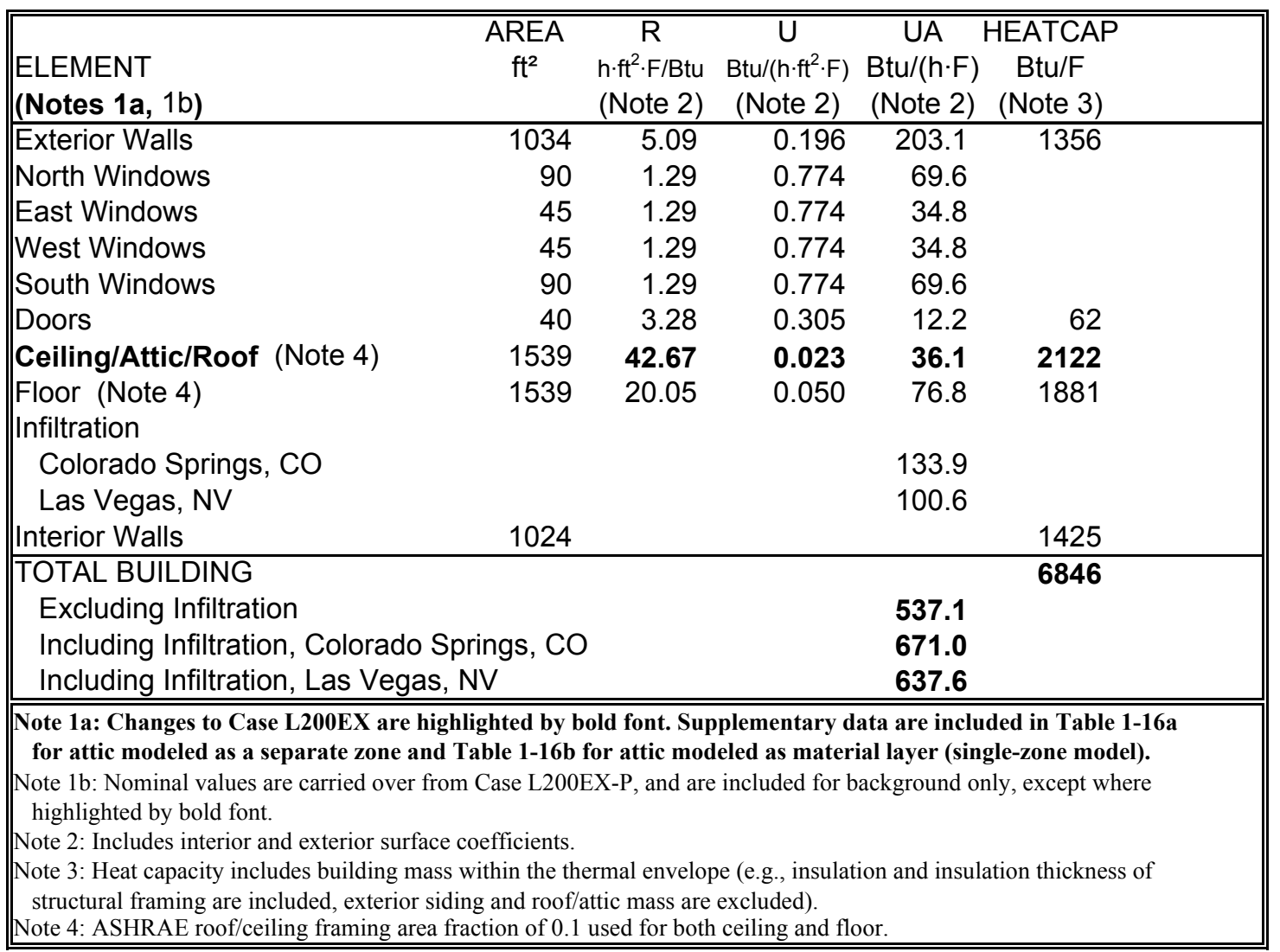

B-EX-Spec.xls, r:a326..g354

02-Feb-10

Table 1-16a. Material Descriptions, Ceiling - Case L220EX

\begin{tabular}{|c|c|c|c|c|c|c|}
\hline $\begin{array}{l}\text { CEILING (inside to outside) } \\
\text { (Notes } 1 \mathrm{a}, 1 \mathrm{~b} \text { ) } \\
\text { ELEMENT }\end{array}$ & Thickness & $\begin{array}{c}\mathrm{R} \\
\mathrm{h} \cdot \mathrm{ft}^{2} \cdot \mathrm{F} / \\
\text { Btu }\end{array}$ & $\begin{array}{c}\mathrm{U} \\
\mathrm{Btu} / \\
\left(\mathrm{h} \cdot \mathrm{ft}^{2} \cdot \mathrm{F}\right) \\
\end{array}$ & $\begin{array}{c}\mathrm{k} \\
\mathrm{Btu} / \\
(\mathrm{h} \cdot \mathrm{ft} \cdot \mathrm{F})\end{array}$ & DENSITY & $\mathrm{Btu} /(\mathrm{lb} \cdot \mathrm{F})$ \\
\hline \multicolumn{7}{|l|}{ CEILING (1539 $\mathrm{ft}^{2}$ total area) } \\
\hline Int Surf Coef & & 0.860 & 1.163 & & & \\
\hline Plasterboard & 0.5 & 0.450 & 2.222 & 0.0926 & 50.0 & 0.26 \\
\hline Fiberglass batt (Note 2) & 3.5 & 11.000 & 0.091 & 0.0265 & 0.6 & 0.20 \\
\hline Blown Cellulose, 2.0"' (Note 2) & 2.0 & 7.229 & 0.138 & 0.0231 & 1.5 & 0.33 \\
\hline Joists $2 \times 6$ 24" O.C. (Note 3 ) & 5.5 & 6.872 & 0.146 & 0.0667 & 32.0 & 0.33 \\
\hline Blown Cellulose, 6.0" (Note 4) & 6.0 & 21.687 & 0.046 & 0.0231 & 1.5 & 0.33 \\
\hline Int Surf Coef & & 0.860 & 1.163 & & & \\
\hline Total air-air, insulated section & & 42.085 & 0.024 & & & \\
\hline Total air-air, framed section & & 30.728 & 0.033 & & & \\
\hline Total air-air, composite section & (Note 5) & 40.585 & 0.025 & & & \\
\hline Total surf-surf, composite sec. & (Note 6) & 38.866 & 0.026 & & & \\
\hline \multicolumn{7}{|c|}{$\begin{array}{l}\text { Note 1a: Changes to Case L200EX are highlighted with bold font. Use this table if attic modeled as separate zone. } \\
\text { Note 1b: Only nominal values are carried over from Case L200EX, and are included for background only except where highlighted by bold font. } \\
\text { Note 2: Insulated section only, see Figure 1-9 for section view of ceiling. } \\
\text { Note 3: Framed section only, see Figure 1-9 for section view of ceiling. } \\
\text { Note 4: This layer of cellulose covers both the framed and insulated sections; see Figure 1-9. } \\
\text { Note 5: Based on } 90 \% \text { insulated section and } 10 \% \text { frame section per ASHRAE; applies to temperature difference between room air and attic air. } \\
\text { Note 6: The "Total surf-surf, composite sec." R-value is the composite air-air R-value less the two interior film coefficient R-values. }\end{array}$} \\
\hline
\end{tabular}

B-EX-Spec.xls, h:a70..i96 12-Aug-10 
Table 1-16b. Material Descriptions for Attic as Material Layer - Case L220EX

\begin{tabular}{|c|c|c|c|c|c|c|}
\hline \multicolumn{7}{|c|}{ COMPOSITE CEILING/ATTIC/ROOF (inside to outside) } \\
\hline \multirow[t]{2}{*}{ (Notes 1a, 1b) } & Thickness & \multirow{2}{*}{$\begin{array}{c}\mathrm{R} \\
\mathrm{h} \cdot \mathrm{ft}^{2} \cdot \mathrm{F} /\end{array}$} & \multirow{3}{*}{$\begin{array}{c}\mathrm{U} \\
\mathrm{Btu} / \\
\left(\mathrm{h} \cdot \mathrm{ft}^{2} \cdot \mathrm{F}\right)\end{array}$} & \multirow{3}{*}{$\begin{array}{c}\mathrm{k} \\
\mathrm{Btu} / \\
(\mathrm{h} \cdot \mathrm{ft} \cdot \mathrm{F})\end{array}$} & \multirow{3}{*}{ 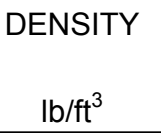 } & \multirow{3}{*}{$\begin{array}{c}\text { Cp } \\
\text { Btu/(lb·F) }\end{array}$} \\
\hline & & & & & & \\
\hline ELEMENT & in. & Btu & & & & \\
\hline \multicolumn{7}{|l|}{ CEILING/ATTIC (1539 $\mathrm{ft}^{2}$ total area) } \\
\hline Int Surf Coef & & 0.860 & 1.163 & & & \\
\hline Plasterboard & 0.5 & 0.450 & 2.222 & 0.0926 & 50.0 & 0.26 \\
\hline Fiberglass batt (Note 2) & 3.5 & 11.000 & 0.091 & 0.0265 & 0.6 & 0.20 \\
\hline Blown Cellulose, 2.0" (Note 2) & 2.0 & 7.229 & 0.138 & 0.0231 & 1.5 & 0.33 \\
\hline Joists 2x6 24" O.C. (Note 3) & 5.5 & 6.872 & 0.146 & 0.0667 & 32.0 & 0.33 \\
\hline Blown Cellulose, 6.0" (Note 4) & 6.0 & 21.687 & 0.046 & 0.0231 & 1.5 & 0.33 \\
\hline Attic air space (Note 5) & & 1.750 & 0.571 & & & \\
\hline Total roof deck/gable, surf-surf (No & 6) & 0.951 & 1.051 & & & \\
\hline Ext Surf Coef (Note 7) & & 0.223 & 4.489 & & & \\
\hline \multicolumn{7}{|l|}{ SUMMARY CEILING/ATTIC/ROOF } \\
\hline Total air-air, insulated section & & 44.150 & 0.023 & & & \\
\hline Total air-air, framed section & & 32.792 & 0.030 & & & \\
\hline Total air-air, composite section & (Note 8) & 42.672 & 0.023 & & & \\
\hline Total surf-surf, composite sec. & (Note 9) & 41.589 & 0.024 & & & \\
\hline \multicolumn{7}{|c|}{ Note 1a: Changes to Case L200EX are highlighted with bold font. Use this table if attic modeled as a material layer. } \\
\hline \multicolumn{7}{|c|}{$\begin{array}{l}\text { Note } 1 \mathrm{~b} \text { : Only nominal values are carried over from Case L200EX, and are included for background only, except where highlighted by bold font } \\
\text { Note 2: Insulated section only, see Figure 1-9 for section view of ceiling. }\end{array}$} \\
\hline \multicolumn{7}{|c|}{ Note 3: Framed section only, see Figure 1-9 for section view of ceiling. } \\
\hline \multicolumn{7}{|c|}{ Note 4: This layer of cellulose covers both the framed and insulated sections; see Figure 1-9. } \\
\hline \multicolumn{7}{|c|}{$\begin{array}{l}\text { Note 5: Average winter/summer values for natural vent (2.4 ACH), R-30 ceiling ins, ext abs = 0.6, includes interior films. } \\
\text { Based on McQuiston and Spitler (1992), Tables } 4.5 \text { and 4.5a, "Effective Resistances of Ceiling or Attic Air Spaces". }\end{array}$} \\
\hline \multicolumn{7}{|c|}{ Note 6: From Table 1-6b (Case L200EX). } \\
\hline \multicolumn{7}{|l|}{ Note 7: Scaled to $1539 \mathrm{ft}^{2}$. } \\
\hline \multicolumn{7}{|c|}{$\begin{array}{l}\text { Note 8: Based on } 10 \% \text { frame area fraction per ASHRAE; applies to temperature difference between room air and ambient air. } \\
\text { Note 9: Based on total air-air R-value less R-values of interior film coefficient and scaled exterior film coefficient. }\end{array}$} \\
\hline
\end{tabular}

B-EX-Spec.xls, h:a99..i132

16-Apr-10

\subsubsection{Case L225EX-P: Wall Insulation Retrofit}

Case L225EX-P is exactly as Case L200EX-P, except that the exterior walls have R-13 blown cellulose insulation as shown in the following figure and tables.

Figure 1-10

Table 1-17

Table 1-18
Exterior wall plan section - Case L225EX

Building Thermal Summary - Case L225EX

Material Descriptions, Exterior Wall - Case L225EX. 


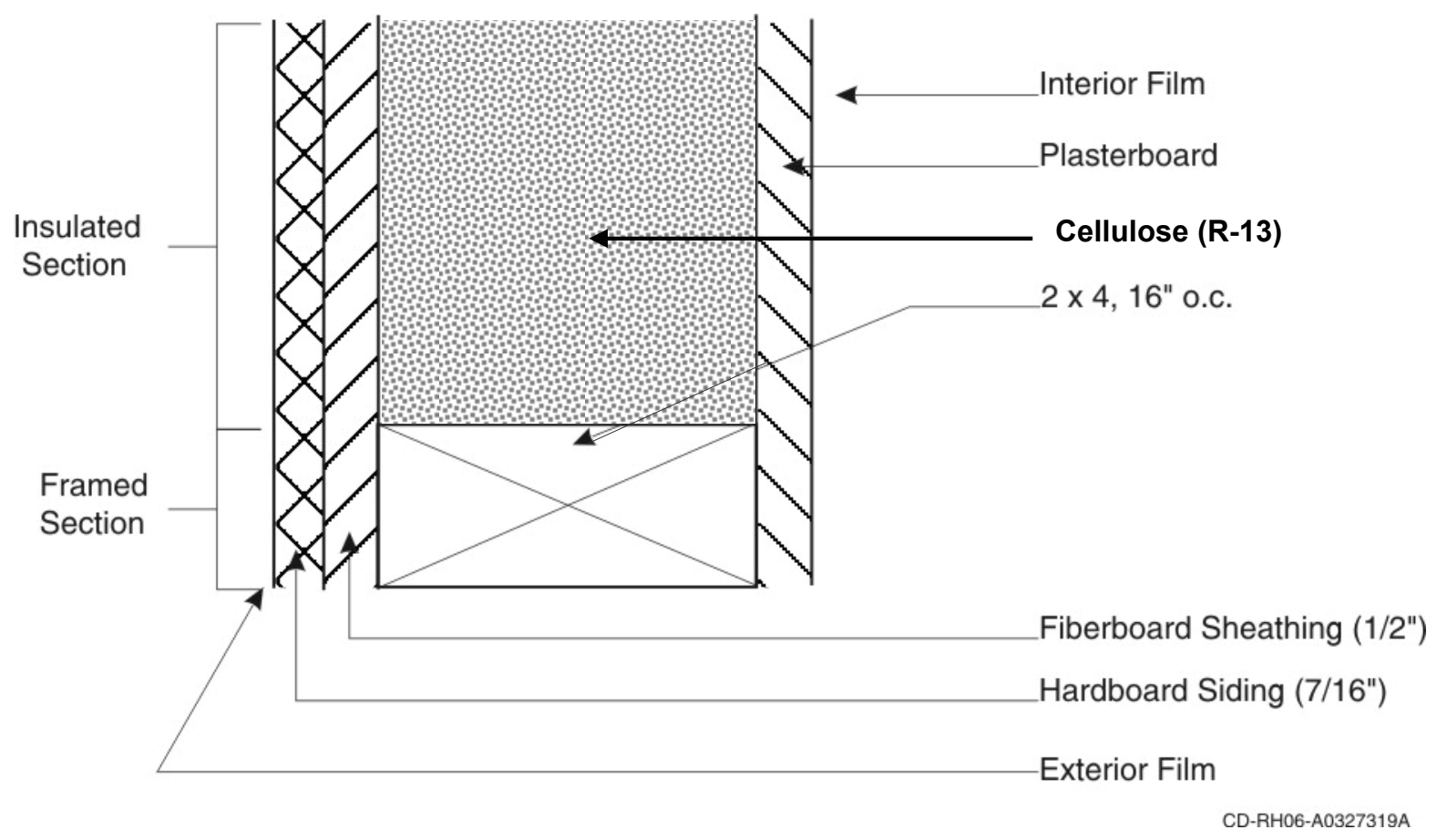

Note: Changes to Case L200EX are highlighted with bold font.

Figure 1-10. Exterior wall plan section - Case L225EX 
Table 1-17. Building Thermal Summary - Case L225EX

\begin{tabular}{|c|c|c|c|c|c|}
\hline $\begin{array}{l}\text { ELEMENT } \\
\text { (Notes 1a, 1b) }\end{array}$ & $\begin{array}{c}\text { AREA } \\
\mathrm{ft}^{2}\end{array}$ & $\begin{array}{c}\mathrm{R} \\
\mathrm{h} \cdot \mathrm{ft}^{2} \cdot \mathrm{F} / \text { Btu } \\
(\text { Note 2) }\end{array}$ & $\begin{array}{c}\mathrm{U} \\
\mathrm{Btu} /\left(\mathrm{h} \cdot \mathrm{ft}^{2} \cdot \mathrm{F}\right) \\
(\text { Note } 2)\end{array}$ & $\begin{array}{c}\text { Btu/ }(\mathrm{h} \cdot \mathrm{F}) \\
\text { (Note 2) }\end{array}$ & $\begin{array}{c}\text { HEATCAP } \\
\text { Btu/F } \\
\text { (Note 3) }\end{array}$ \\
\hline Exterior Walls (Note 4) & 1034 & 13.00 & 0.077 & 79.6 & 1618 \\
\hline North Windows & 90 & 1.29 & 0.774 & 69.6 & \\
\hline East Windows & 45 & 1.29 & 0.774 & 34.8 & \\
\hline West Windows & 45 & 1.29 & 0.774 & 34.8 & \\
\hline South Windows & 90 & 1.29 & 0.774 & 69.6 & \\
\hline Doors & 40 & 3.28 & 0.305 & 12.2 & 62 \\
\hline Ceiling/Attic/Roof (Note 5) & 1539 & 13.67 & 0.073 & 112.6 & 1356 \\
\hline Floor (Note 5) & 1539 & 20.05 & 0.050 & \multirow[t]{2}{*}{76.8} & 1881 \\
\hline Infiltration & & & & & \\
\hline Colorado Springs, CO & & & & 133.9 & \\
\hline Las Vegas, NV & & & & \multirow[t]{2}{*}{100.6} & \\
\hline Interior Walls & 1024 & & & & 1425 \\
\hline \multicolumn{3}{|l|}{ |TOTAL BUILDING } & & \multicolumn{2}{|r|}{1} \\
\hline \multicolumn{3}{|l|}{ Excluding Infiltration } & & 490.0 \\
\hline \multicolumn{3}{|c|}{ Including Infiltration, Colorado Springs, CO } & \multicolumn{3}{|c|}{623.9} \\
\hline \multicolumn{3}{|c|}{ Including Infiltration, Las Vegas, NV } & \multicolumn{2}{|r|}{590.6} & \\
\hline \multicolumn{6}{|c|}{$\begin{array}{l}\text { Note 1a: Changes to Case L200EX are highlighted by bold font. } \\
\text { Note 1b: Only nominal values are carried over from Case L200EX, and are included for background only, except where } \\
\text { highlighted by bold font. } \\
\text { Note 2: Includes interior and exterior surface coefficients. } \\
\text { Note 3: Heat capacity includes building mass within the thermal envelope (e.g., insulation and insulation thickness of } \\
\text { structural framing are included, exterior siding and roof/attic mass are excluded). } \\
\text { Note 4: Excludes window and door areas. ASHRAE framed area fraction of } 0.25 \text { used for } 2 \times 4 \text { 16" O.C. construction. } \\
\text { Note 5: ASHRAE roof/ceiling framing area fraction of } 0.1 \text { used for both ceiling and floor. }\end{array}$} \\
\hline
\end{tabular}

B-EX-Spec.xls, r:a362..g391

02-Feb-10

Table 1-18. Material Descriptions, Exterior Wall - Case L225EX

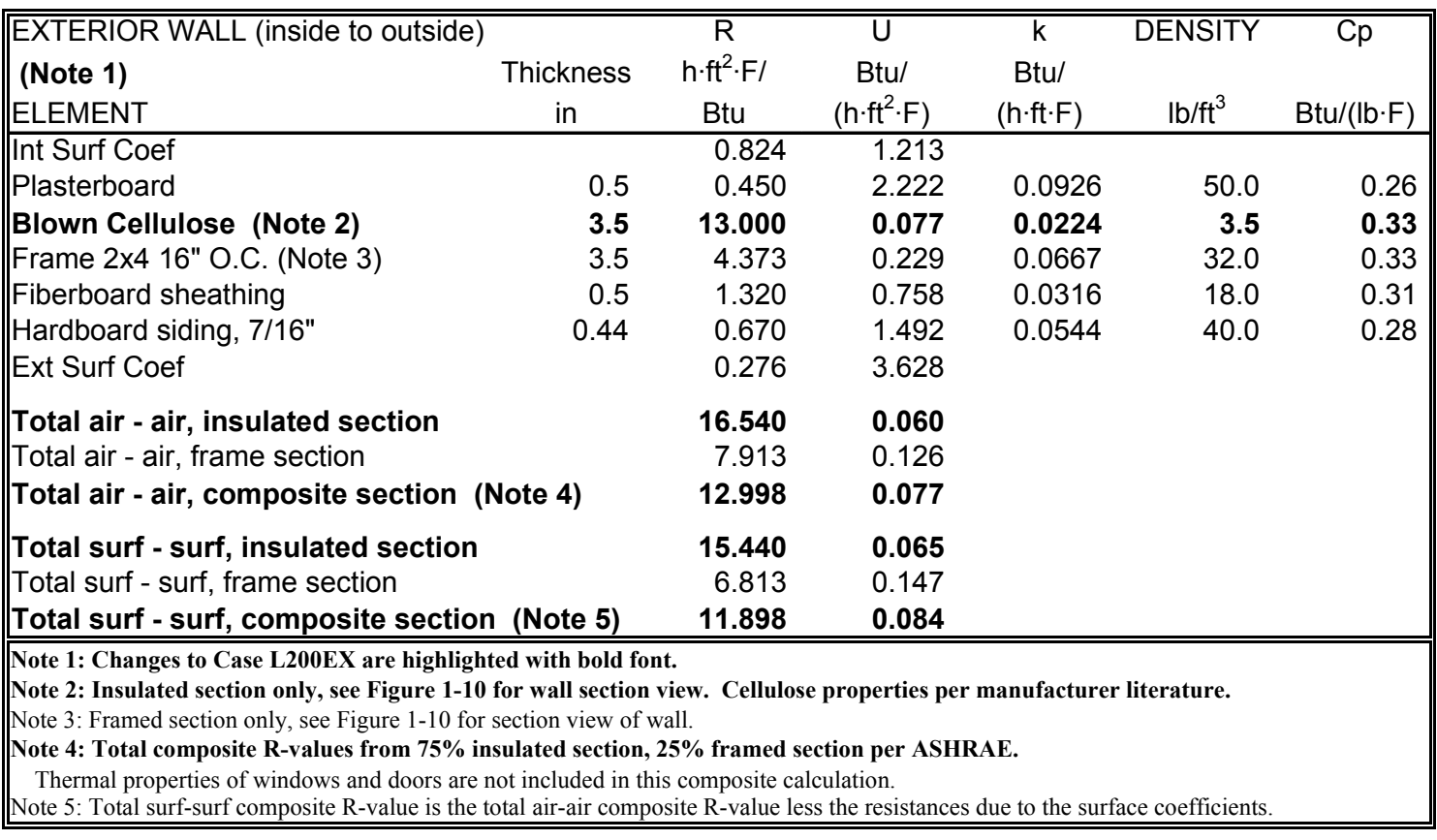




\subsubsection{Case L240EX-P: Programmable Thermostat Retrofit}

Case L240EX-P is exactly as Case L200EX-P, except for changes described in Sections 1.2.2.4.1 and 1.2.2.4.2.

\subsection{Colorad.TM2}

For Colorad.TM2 weather data (heating only), thermostat setback is applied on all nights during the heating season from 10:00 p.m. to 6:00 a.m. as shown below.

During heating season (October 7 - May 16):

$$
\begin{aligned}
& \text { 10:00 p.m. }-6: 00 \text { a.m.: HEAT }=\text { ON IF TEMP }<\mathbf{6 2}^{\circ} \mathbf{F} ; \mathrm{COOL}=\mathrm{OFF} \\
& \text { 6:00 a.m. }-10: 00 \text { p.m.: HEAT }=\text { ON IF TEMP }<68^{\circ} \mathrm{F} ; \mathrm{COOL}=\mathrm{OFF}
\end{aligned}
$$

During non-heating season (May 17 - October 6):

$$
\mathrm{HEAT}=\mathrm{OFF} ; \mathrm{COOL}=\mathrm{OFF} \text {. }
$$

Where:

- “TEMP” refers to conditioned zone-air temperature.

- The heating season start/stop dates are the same as for Case L200EX-P.

\subsection{Lasvega.TM2}

For Lasvega.TM2 weather data (cooling only) thermostat setup is applied on all days from 8:00 a.m. to 5:00 p.m. as shown below.

During cooling season (March 28 - October 28):

$$
\begin{aligned}
& \text { 8:00 a.m. }-5: 00 \text { p.m.: COOL }=\text { ON IF TEMP }>\mathbf{8 4}^{\circ} \mathbf{F} ; \text { HEAT }=\text { OFF } \\
& \text { 5:00 p.m. }-8: 00 \text { a.m.: COOL }=\text { ON IF TEMP }>78^{\circ} \text { F } ; \text { HEAT }=\text { OFF }
\end{aligned}
$$

During non-cooling season (October 29 - March 27):

$$
\mathrm{COOL}=\mathrm{OFF} ; \mathrm{HEAT}=\mathrm{OFF} \text {. }
$$

Where:

- "TEMP" refers to conditioned zone-air temperature.

- The cooling season start/stop dates are the same as for Case L200EX-P.

\subsubsection{Case L250EX-P: Double-Pane Low-Emissivity Window With Wood Frame Retrofit}

Case L250EX-P is exactly as Case L200EX-P, except that all single-pane windows are replaced with double-pane low-emissivity (low-e) windows with wood frames and insulated spacers. Window and frame geometry remain as for Case L200EX-P. The following tables specify basic properties of the window, including shading coefficient (SC), solar heat gain coefficient (SHGC), and thermal resistance:

Table 1-19

Table 1-20

Table 1-21

Table 1-22

Table 1-23
Building Thermal Summary - Case L250EX

Window Summary (Double-Pane, Low-E, Argon Fill, Wood Frame, Insulated Spacer) - Case L250EX

Low-E Glazing System With Argon Gas Fill Glazing Summary (Center of Glass Values) - Case L250EX

Optical Properties as a Function of Incidence Angle for Low-Emissivity DoublePane Glazing - Case L250EX

Component Solar Fractions - Case L250EX. 
Use only the information that is relevant to the program being tested. Window properties are drawn from WINDOW 5.2 (2005) software for window thermal analysis (see Appendix E). For programs that need transmittance or reflectance at other angles of incidence, interpolate between the values of Table 1-22 using the cosine of the incidence angle as the basis of interpolation. Where other unspecified data are needed, values that are consistent with those quoted must be calculated.

There is a slight change in interior surface solar distribution caused by reduced solar lost (cavity albedo); for tools that can vary this input, values are included in Table 1-23.

Note for highlighting of changes in Tables 1-19 through 1-23.

- Because of the large number of changes to the window for this case, changes to Case L200EX are not highlighted; rather, bold font in Tables 1-20, 1-21, and 1-22 highlights changes to HERS BESTEST Case L130A window inputs only (as a convenience for those who have previously run HERS BESTEST).

- Bold font in Tables 1-19 and 1-23 highlights changes to Case L200EX (usual convention). 
Table 1-19. Building Thermal Summary - Case L250EX

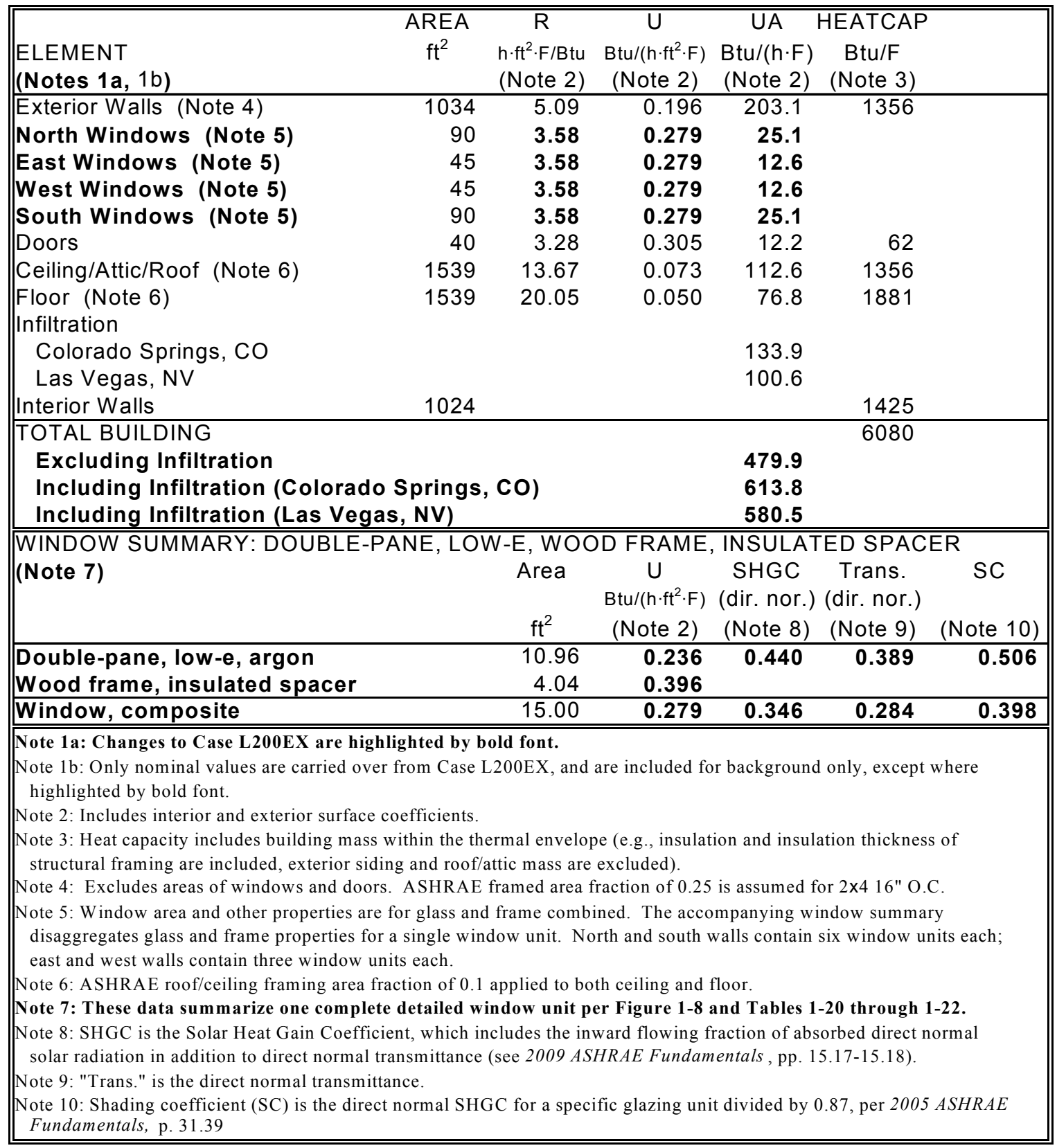



Table 1-20. Window Summary (Double-Pane, Low-E, Argon Fill, Wood Frame,
Insulated Spacer) - Case L250EX

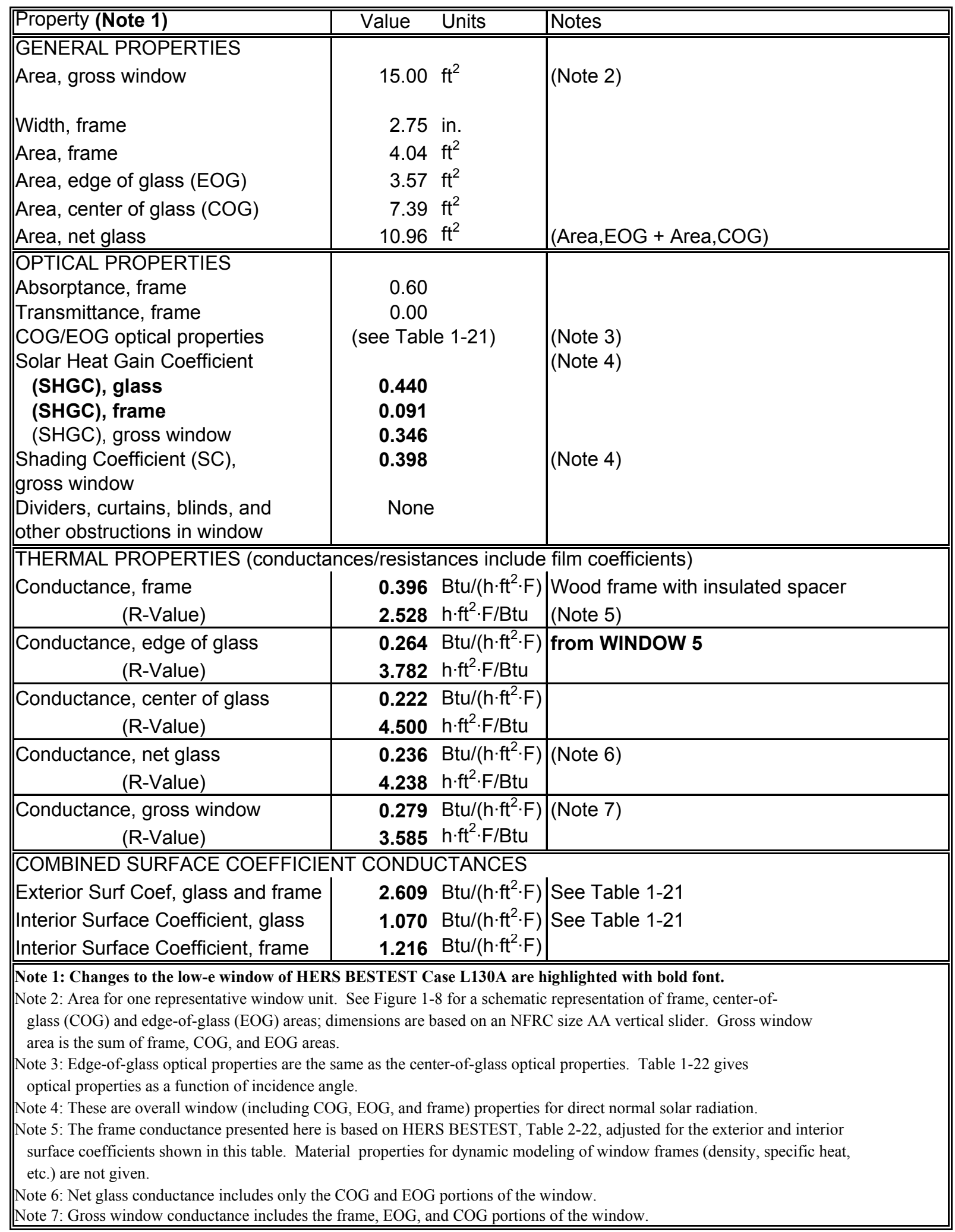

B-EX-Spec, L:a68..h118; 
Table 1-21. Low-E Glazing System With Argon Gas Fill Glazing Summary (Center of Glass Values) - Case L250EX

\begin{tabular}{|c|c|c|}
\hline Property & Value & Notes \\
\hline $\begin{array}{l}\text { GENERAL PROPERTIES } \\
\text { Number of Panes } \\
\text { Pane Thickness } \\
\text { Argon Gap Thickness }\end{array}$ & $\begin{array}{l}2.000 \\
0.118 \text { in } \\
0.500 \text { in }\end{array}$ & (Note 1) \\
\hline $\begin{array}{l}\text { OUTER PANE OPTICAL PROP. } \\
\text { Transmittance } \\
\text { Reflectance (outside facing surf.) } \\
\text { Reflectance (inside facing surf.) } \\
\text { Absorptance } \\
\text { Index of Refraction } \\
\text { Extinction Coefficient }\end{array}$ & $\begin{array}{l}0.450 \\
0.340 \\
0.370 \\
0.210\end{array}$ & \begin{tabular}{|l} 
\\
(Note 3) \\
(Note 3)
\end{tabular} \\
\hline $\begin{array}{l}\text { INNER PANE OPTICAL PROP. } \\
\text { Transmittance } \\
\text { Reflectance } \\
\text { Absorptance } \\
\text { Index of Refraction } \\
\text { Extinction Coefficient }\end{array}$ & $\begin{array}{l}0.837 \\
0.075 \\
\mathbf{0 . 0 8 9} \\
1.5223 \\
0.7806 \text { /in }\end{array}$ & \\
\hline \begin{tabular}{|l|} 
DOUBLE PANE OPTICAL PROP. \\
Transmittance \\
Reflectance,f \\
Reflectance,b \\
Absorptance (outer pane) \\
Absorptance (inner pane) \\
Solar Heat Gain Coefficient (SHGC) \\
Shading Coefficient (SC) \\
Optical Properties as a Function \\
of Incident Angle \\
\end{tabular} & $\begin{array}{l}0.389 \\
0.350 \\
0.337 \\
0.219 \\
0.041 \\
0.440 \\
0.506 \\
\text { (See Table 1-22) }\end{array}$ & $\begin{array}{l}\text { (Note 4) } \\
\text { (Note 4) }\end{array}$ \\
\hline $\begin{array}{l}\text { THERMAL PROPERTIES } \\
\text { Conductivity of Glass }\end{array}$ & $0.520 \mathrm{Btu} /(\mathrm{h} \cdot \mathrm{ft} \cdot \mathrm{F})$ & \\
\hline $\begin{array}{l}\text { Combined Radiative and Convec- } \\
\text { tive Coefficient of Argon Gap } \\
\text { (R-Value) }\end{array}$ & $\begin{array}{l}0.318 \mathrm{Btu} /\left(\mathrm{h} \cdot \mathrm{ft}^{2} \cdot \mathrm{F}\right) \\
3.144 \mathrm{~h} \cdot \mathrm{ft}^{2} \cdot \mathrm{F} / \mathrm{Btu}\end{array}$ & (Note 6) \\
\hline $\begin{array}{c}\text { Conductance of Glass Pane } \\
\text { (R-Value) }\end{array}$ & $\begin{array}{rl}52.881 & \mathrm{Btu} /\left(\mathrm{h} \cdot \mathrm{ft}^{2} \cdot \mathrm{F}\right) \\
0.019 & \mathrm{~h} \cdot \mathrm{ft}^{2} \cdot \mathrm{F} / \mathrm{Btu}\end{array}$ & \\
\hline $\begin{array}{c}\text { Exterior Combined Surface Coef. } \\
\text { (R-Value) }\end{array}$ & $\begin{array}{ll}2.609 & \mathrm{Btu} /\left(\mathrm{h} \cdot \mathrm{ft}^{2} \cdot \mathrm{F}\right) \\
0.383 & \mathrm{~h} \cdot \mathrm{ft}^{2} \cdot \mathrm{F} / \mathrm{Btu}\end{array}$ & \\
\hline $\begin{array}{c}\text { Interior Combined Surface Coef. } \\
\text { (R-Value) }\end{array}$ & $\begin{array}{ll}1.070 & \mathrm{Btu} /\left(\mathrm{h} \cdot \mathrm{ft}^{2} \cdot \mathrm{F}\right) \\
0.935 & \mathrm{~h} \cdot \mathrm{ft}^{2} \cdot \mathrm{F} / \mathrm{Btu}\end{array}$ & \\
\hline $\begin{array}{r}\text { U-Value, Air-Air } \\
\text { (R-Value) }\end{array}$ & $\begin{array}{ll}0.222 & \mathrm{Btu} /\left(\mathrm{h} \cdot \mathrm{ft}^{2} \cdot \mathrm{F}\right) \\
4.500 & \mathrm{~h} \cdot \mathrm{ft}^{2} \cdot \mathrm{F} / \mathrm{Btu}\end{array}$ & \\
\hline $\begin{array}{l}\text { Hemispherical Infrared Emittance } \\
\text { Infrared Transmittance } \\
\text { Density of Glass } \\
\text { Specific Heat of Glass }\end{array}$ & $\begin{array}{rl}0.84 & \\
0 & \\
154 & \mathrm{lb} / \mathrm{ft}^{3} \\
0.18 & \mathrm{Btu} /(\mathrm{lb} \cdot \mathrm{F})\end{array}$ & (Note 2) \\
\hline \multicolumn{3}{|c|}{$\begin{array}{l}\text { Note 1: Changes to low-e window of HERS BESTEST Case L130A highlighted with bold for } \\
\text { Note 2: Optical properties listed in this table are for direct normal radiation. The inside facing } \\
\text { surface of the outer pane has emissivity = 0.04. } \\
\text { Note 3: Single values of index of refraction and extinction coefficient do not adequately } \\
\text { describe the optical properties of coated glass. } \\
\text { Note 4: Reflectance,f and Reflectance,b are overall solar reflectances for radiation incident } \\
\text { from the front (from the outside) and back (from the inside), respectively. } \\
\text { Note 5: SC = SHGC/0.87 per Eqn. 91 (p. 31.39) of ASHRAE 2005 Fundamentals. } \\
\text { Note 6: Calculated from WINDOW } 5 \text { output; Ugap = (Utot)(dTtot)/(dTgap), } \\
\text { for center of glass values at winter design conditions. }\end{array}$} \\
\hline
\end{tabular}


Table 1-22. Optical Properties as a Function of Incidence Angle for Low-Emissivity Double-Pane Glazing - Case L250EX

\begin{tabular}{|c|c|c|c|c|c|c|}
\hline \multirow[b]{2}{*}{ Angle } & \multicolumn{5}{|c|}{ Properties (Notes 1a, 1b, 2, 3) } & \multirow[b]{2}{*}{ SHGC } \\
\hline & Trans & Refl,f & Refl,b & Abs Out & Abs In & \\
\hline 0 & 0.389 & 0.350 & 0.337 & 0.219 & 0.041 & 0.440 \\
\hline 10 & 0.389 & 0.350 & 0.337 & 0.219 & 0.041 & 0.440 \\
\hline 20 & 0.384 & 0.349 & 0.335 & 0.226 & 0.042 & 0.437 \\
\hline 30 & 0.376 & 0.351 & 0.336 & 0.231 & 0.042 & 0.429 \\
\hline 40 & 0.366 & 0.359 & 0.341 & 0.232 & 0.043 & 0.420 \\
\hline 50 & 0.346 & 0.373 & 0.355 & 0.236 & 0.044 & 0.401 \\
\hline 60 & 0.305 & 0.402 & 0.388 & 0.250 & 0.043 & 0.360 \\
\hline 70 & 0.226 & 0.471 & 0.470 & 0.264 & 0.038 & 0.279 \\
\hline 80 & 0.107 & 0.639 & 0.645 & 0.224 & 0.029 & 0.149 \\
\hline 90 & 0.000 & 0.999 & 1.000 & 0.001 & 0.000 & 0.000 \\
\hline Hemis & 0.323 & 0.391 & 0.378 & 0.235 & 0.041 & 0.376 \\
\hline \multicolumn{7}{|c|}{$\begin{array}{l}\text { Note 1a: Changes to low-e window of HERS BESTEST Case L130A are highlighted with bold font. } \\
\text { Note 1b: Updates to incident-angle dependent transmittance, reflectances, and absorptances are } \\
\text { for WINDOW } 5 \text { (WINDOW } 4.1 \text { was used for HERS BESTEST). } \\
\text { Note 2: Trans = Transmittance; } \\
\text { Refl,f = Overall solar reflectance for radiation incident from the front (i.e., from the outside); } \\
\text { Refl,b = Overall solar reflectance for radiation incident from the back (i.e., from inside the zone); } \\
\text { Abs Out = absorptance of outer pane; Abs In = absorptance of inner pane; SHGC = solar heat gain coeft } \\
\text { Hemis = hemispherically integrated property. Transmittance, reflectance, and SHGC are overall } \\
\text { properties for the glazing system (inside pane, argon fill, and outer pane), excluding the frame. } \\
\text { Note 3: SHGC is from Equations 18, 19, and } 21 \text { (pp. 15.17-18) of ASHRAE } 2009 \text { Fundamentals. }\end{array}$} \\
\hline
\end{tabular}

B-EX-Spec, b!:a148..h175 
Table 1-23. Component Solar Fractions - Case L250EX

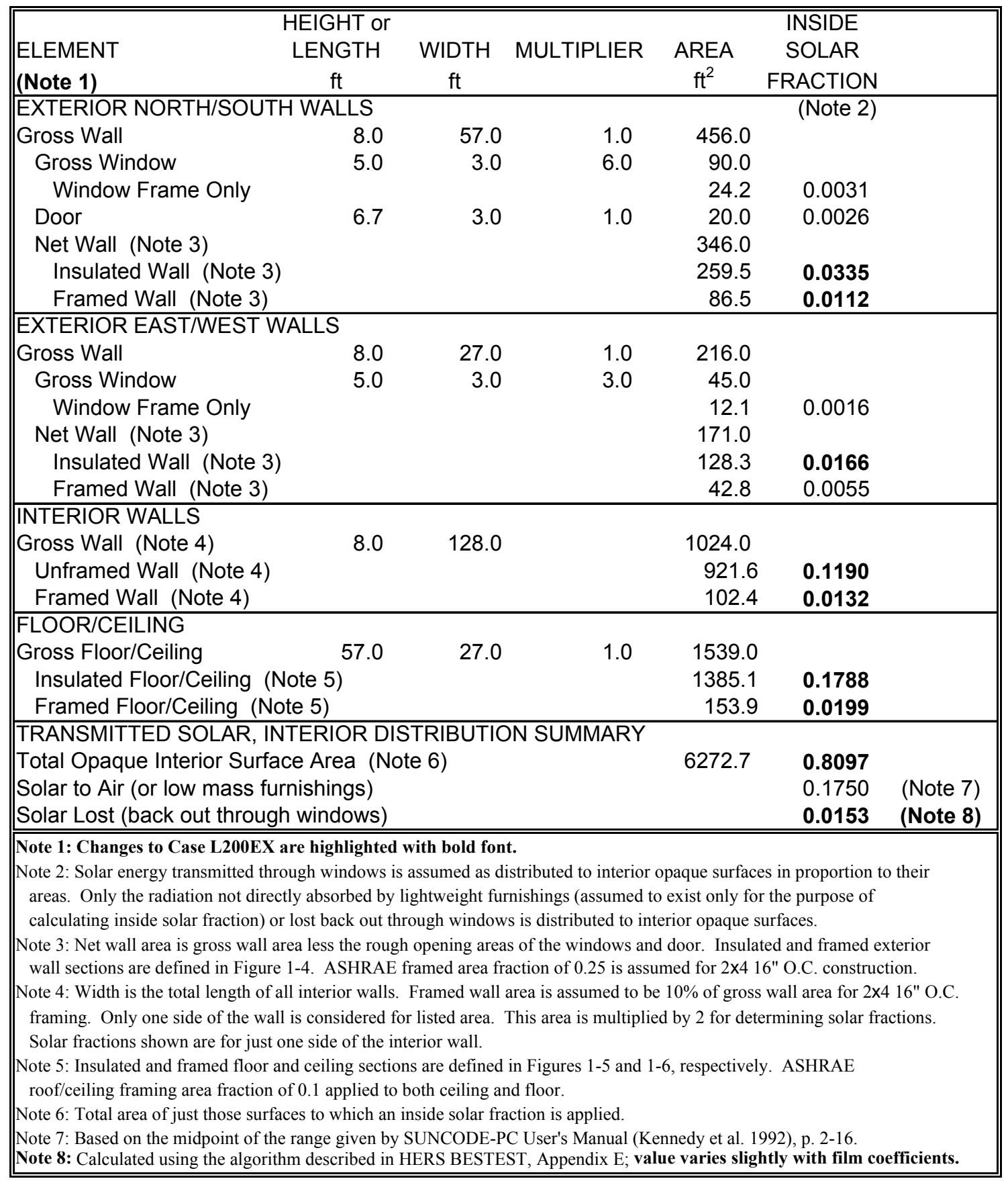




\subsubsection{Case L260EX-P: High Solar Absorptance Roof (Cool Roof Base Case)}

Case L260EX-P is exactly as Case L200EX-P, except that exterior shortwave (visible and UV) absorptance $\left(\alpha_{\text {ext }}\right)$ is 0.8 for the roof only.

\subsubsection{Case L265EX-P: Low Solar Absorptance Roof (Cool Roof)}

Case L265EX-P is exactly as Case L200EX-P, except that exterior shortwave (visible and UV) absorptance $\left(\alpha_{\text {ext }}\right)$ is 0.2 for the roof only.

\subsubsection{Case L270EX-P: External Shading}

Case L270EX-P is exactly as Case L200EX-P, except for the following changes:

- An opaque overhang is included at the top of the south, east, and west exterior walls. The overhang extends outward from this wall $6.0 \mathrm{ft}$ (see Figures 1-11 and 1-12). Window horizontal spacing along the walls is shown previously in Figure 1-2. The overhang traverses the entire length of the south, east, and west walls.

- External shading device (overhang) optical properties:

- Solar absorptance $=1$ (reflectance $=0$, transmittance $=0$ ) independent of incidence angle .

$\circ \quad$ Infrared emittance $=0$.

- Apply these values as nearly as the program being tested allows.

- All heat from solar radiation absorbed by the shading devices is dissipated to the ambient environment via convection.

- The properties listed above apply to both sides of the shading devices.

○ If the program being tested does not allow variation of these properties, use its default values.

- Thickness: If the program requires an input for thickness of shading devices, use the smallest allowable value (e.g., $0.001 \mathrm{~m})$.

Recall from Section 1.1 that this test requires use of consistent modeling methods for the test cases. 


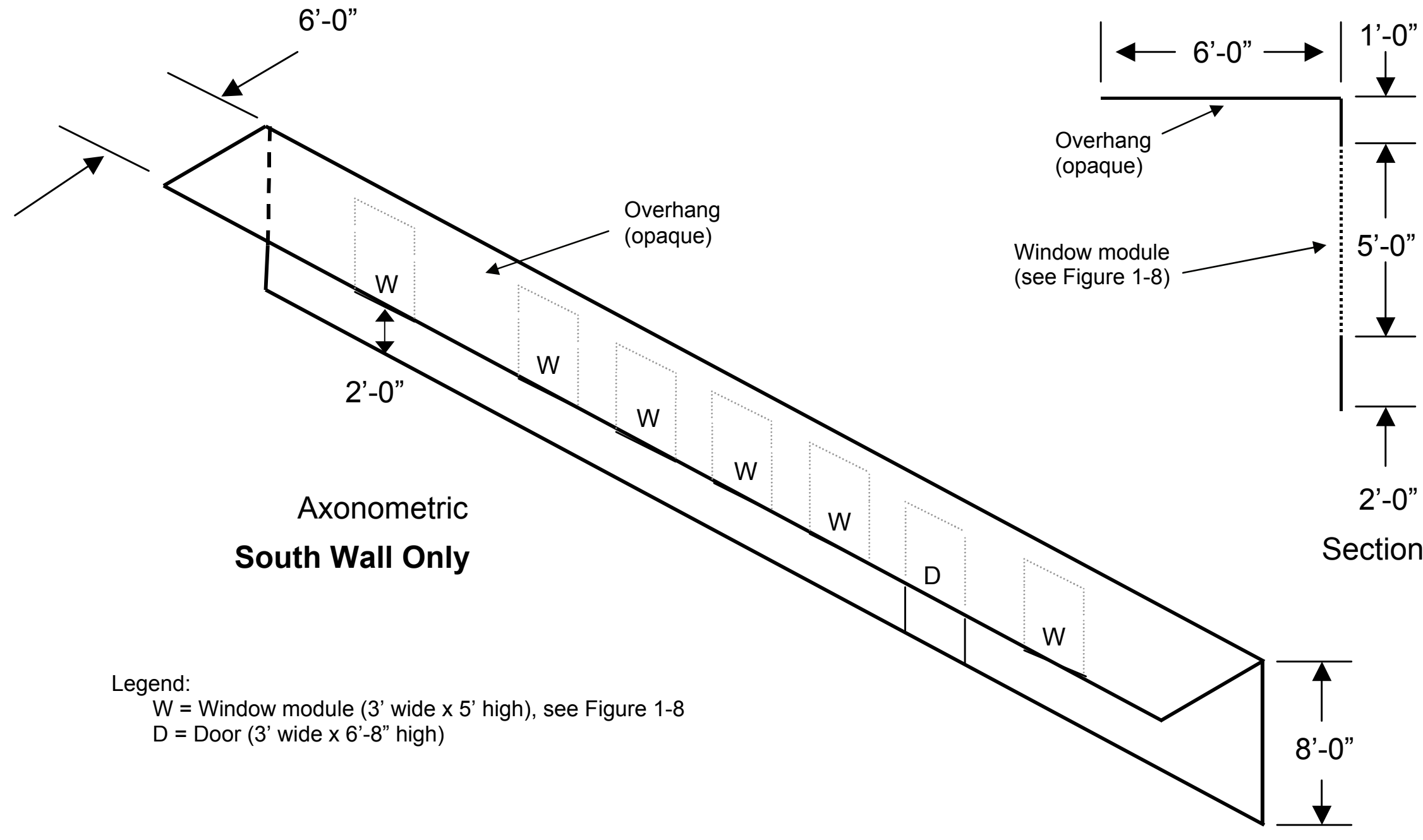

Figure 1-11. South overhang - Case L270EX-P 


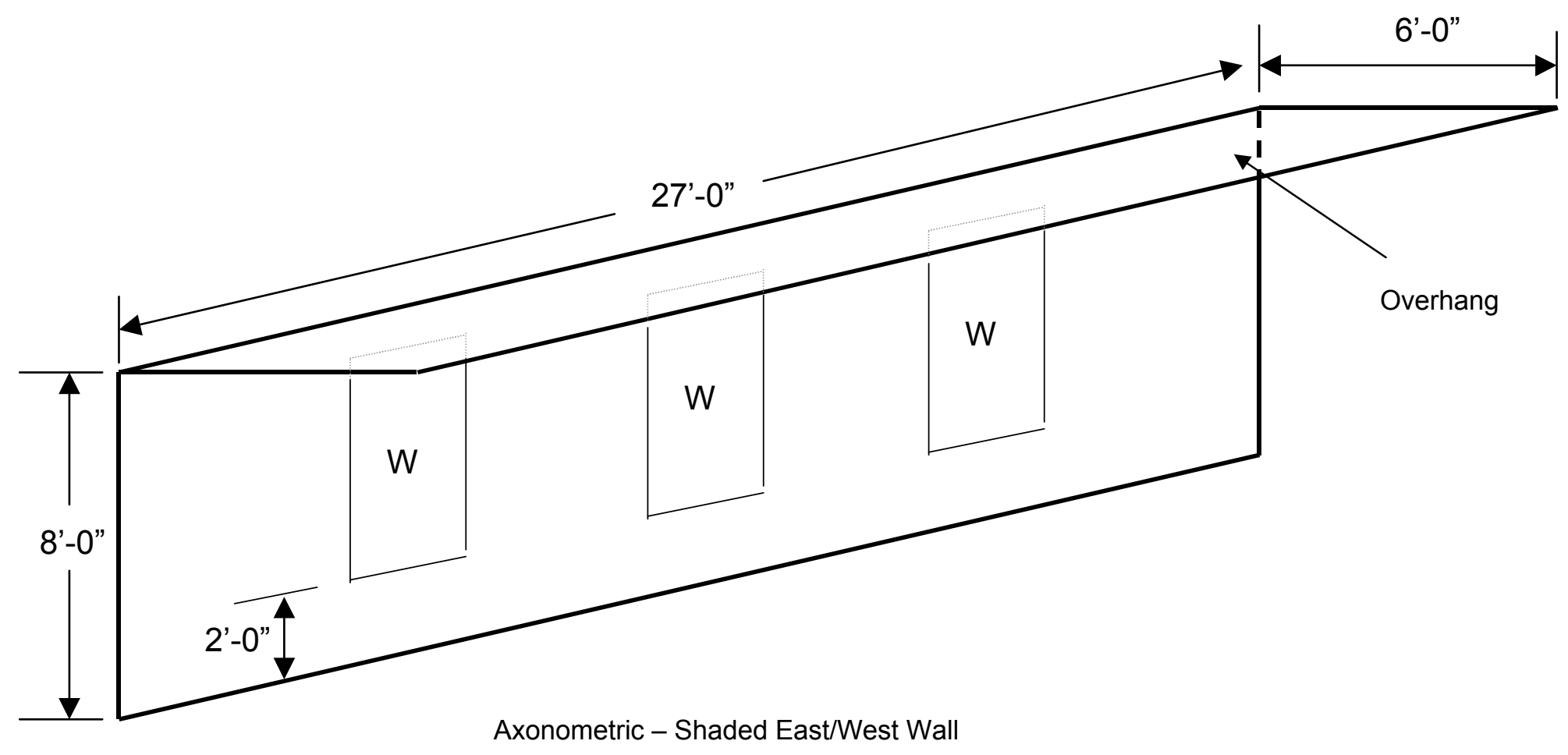

Legend:

- $\quad W=$ Typical window module $\left(3^{\prime}\right.$ wide $\times 5^{\prime}$ high), see Figure 1-8.

Figure 1-12. Overhang for east and west windows - Case L270EX-P 


\subsubsection{Case L300EX-P: Combined Retrofits}

Case L300EX-P is exactly as Case L200EX-P, except for the following changes.

\subsection{Case L300EX-PH}

For the heating climate (Colorad.TM2 weather) include:

- Air-seal retrofit as described in Section 1.2.2.1

- Attic insulation retrofit as described in Section 1.2.2.2

- Wall insulation retrofit as described in Section 1.2.2.3

- Programmable thermostat retrofit as described in Section 1.2.2.4

- Low-e window retrofit as described in Section 1.2.2.5.

\subsection{Case L300EX-PC}

For the cooling climate (Lasvega.TM2 weather) include:

- All the retrofits listed in Section 1.2.2.9.1

- The low solar absorptance roof retrofit as described in Section 1.2.2.7

- The external shading retrofit as described in Section 1.2.2.8.

\subsection{Calibrated Energy Savings Tests Input Specifications}

Run the calibrated energy savings test cases after the building physics test cases of Section 1.2 are complete, with all Section 1.2 results disagreements diagnosed and all found modeling errors corrected. Correction of modeling errors must have a mathematical, physical, or logical basis and must be applied consistently throughout the test cases. Some disagreements may have a logical basis (i.e., may be based on legitimate modeling differences).

The Section 1.3 test cases are based on the Section 1.2 test cases with changes noted in the following sections. Except where noted, figures and tables for the building physics base case (L200EX-P) are applied for developing the calibrated base case (L200EX-C).

Section 1.3 is written such that: a) a preliminary non-calibrated base-case model is developed as described in Section 1.3.1, b) inputs for the base-case simulation model (see Section 1.3.1) are calibrated using reference utility energy-use data given in Section 1.3.1.2, and c) inputs for retrofit cases (see Section 1.3.2) are developed using calibrated base-case inputs with modifications as specified for the given retrofit cases. Some modeling methods may calculate calibrated energy savings, without adjustment to model inputs, e.g., by comparing differences between base-case utility billing data versus predicted noncalibrated base-case energy use, and then applying an appropriate adjustment to predicted non-calibrated energy savings. For programs that apply methods not requiring adjustment to base-case model inputs, use the utility bills called out in Section 1.3.1.2 for calibration, however, specific instructions of Section 1.3.1.2 (and elsewhere in Section 1.3) regarding adjustment of inputs for calibration do not apply.

\subsubsection{Pre-Retrofit Base-Case Building (Case L200EX-C)}

Case L200EX-C is exactly as Case L200EX-P, except for changes described in the following subsections.

\subsubsection{Approximate Inputs}

Approximate input ranges are provided for selected base-case model input parameters listed in Section 1.3.1.2. The selected parameters strongly affect energy use predictions and are commonly known to have pre-retrofit audit uncertainty. For calibrating the pre-retrofit base case to reference utility energy-use data, variation of approximate inputs is allowed during the model calibration phase, as described in Section 1.3.1.2. The following nomenclature is used to denote approximate input ranges in the data tables: 
- "Min": approximate input range minimum value

- "Max": approximate input range maximum value.

For parameters with approximate input ranges, the nominal values provided in data tables are for use with the Section 1.2 building physics test cases, and as described in Section 1.3.1.2.

Where approximate input ranges are provided, explicit inputs for reference programs are selected from within the approximate input range as described in Appendix F. Symmetric or asymmetric triangular probability distribution is assumed as appropriate within the given approximate input ranges for randomly selecting explicit inputs used for developing reference utility energy use data.

\subsubsection{Calibration of Pre-Retrofit Base-Case Inputs}

- For programs (or program modes) not requiring use of utility billing data to run a simulation, use the nominal inputs and results for Case L200EX-P (see Section 1.2.1) as the uncalibrated simulation. For programs (or program modes) requiring use of utility billing data, use the nominal inputs (see Section 1.2.1) as applicable for uncalibrated inputs.

- For the Colorad.TM2 heating only climate, calibrate the base-case approximate inputs using the reference utility energy-use data given in Tables 1-24a through 1-24g for the following base-case calibration scenarios.

- Table 1-24a, Case L200EX-C1H, targeted high space heating use

- Table 1-24b, Case L200EX-C2H, targeted low space heating use

- Table 1-24c, Case L200EX-C3H, fully random selection, near-nominal space heating use

- Table 1-24d, Case L200EX-C4H, fully random selection, high space heating use

- Table 1-24e, Case L200EX-C5H, fully random selection, low space heating use

- Table 1-24f, Case L200EX-C6H, fully random selection, mid-high space heating use

○ Table 1-24g, Case L200EX-C7H, fully random selection, mid-low space heating use.

- For the Lasvega.TM2 cooling only climate, calibrate the base-case approximate inputs using the reference utility energy-use data given in Tables 1-25a through 1-25g for the following base-case calibration scenarios.

- Table 1-25a, Case L200EX-C1C, targeted high space cooling use

- Table 1-25b, Case L200EX-C2C, targeted low space cooling use

- Table 1-25c, Case L200EX-C3C, fully random selection, near-nominal space cooling use

- Table 1-25d, Case L200EX-C4C, fully random selection, high space cooling use

$\circ$ Table 1-25e, Case L200EX-C5C, fully random selection, low space cooling use

- Table 1-25f, Case L200EX-C6C, fully random selection, mid-high space cooling use

- Table 1-25g, Case L200EX-C7C, fully random selection, mid-low space cooling use.

- Use the calibration method typically applied by your software; this test procedure does not provide guidance for calibration methodologies. Only approximate inputs related to the model parameters listed below are allowed to be varied for calibration.

- Exterior wall R-value (see Tables 1-1 and 1-4)

- For calibrating the exterior wall inputs, assume the empty cavity (air gap) Rvalue and wood-framing material properties are explicitly known; vary material properties of exterior siding/sheathing and/or interior wall materials only. This allows development of a clearer relative retrofit effect in Case L225EX-C. To develop reference simulation program base-case explicit inputs, only the exterior hardboard siding thickness was randomly varied as shown in Table 1-4.

- Composite ceiling/attic/roof R-value (see Tables 1-1, 1-6a, and 1-6b) 
- To develop reference simulation program base-case explicit inputs, the batt insulation (and modeled joist) thicknesses were randomly varied (see Tables 1-6a and 1-6b). Attic joist thickness varies with insulation thickness. However, the maximum modeled attic joist thickness is 5.5 in.; modeled batt insulation thickness may be slightly greater than 5.5 in.

- Infiltration rate or leakage area, etc. (see Tables 1-2, 1-8a, 1-8b, and Section 1.3.1.3.1)

- Internal gains and related fractions of base load energy use corresponding to sensible internal gains (see Tables 1-2, 1-9a, 1-9b, and Section 1.3.1.3.2)

- Exterior solar absorptance (see Table 1-2 and Section 1.3.1.3.3)

- Thermostat heating and cooling set points (see Section 1.3.1.3.4)

○ Thermostat heating and cooling season start/stop dates (see Section 1.3.1.3.4)

○ Furnace efficiency (see Section 1.3.1.3.5)

○ Mechanical cooling COP (see Section 1.3.1.3.5).

- Develop calibrated approximate inputs independently for each space heating and space cooling base-case scenario. Model parameters listed above are applicable to both space heating and space cooling scenarios, except heating set point, heating season start/stop dates, and furnace efficiency apply to space-heating cases only; cooling set point, cooling season start/stop dates, and cooling COP apply to space-cooling cases only.

- It may be reasonable for calibrated inputs to vary slightly outside of specified approximate input ranges because randomly selected explicit inputs for the reference simulations can occur near the extremes of a given approximate input range.

- Use the new base-case models with the calibrated approximate inputs to develop the input files for the retrofit cases of Section 1.3.2.

Reference energy use data provided in Tables 1-24a through 1-24g and Tables 1-25a through 1-25g are the average of the results for the reference simulation models using EnergyPlus, SUNREL, and DOE-2.1E. The reference simulations apply explicit inputs randomly selected from within the given approximate input ranges (see Appendix F). All reference simulation explicit inputs are selected independently for each space-heating and space-cooling base-case scenario, except heating thermostat settings/schedule and furnace efficiency are only selected for space heating cases, and cooling thermostat settings/schedule and cooling COP are only selected for space cooling cases. The reference simulation explicit inputs are intended to be unknown for the software being tested and are not given in the test specification. Thirteen months of base-case energy use data are provided as recommended by the BESTEST-EX Working Group (2009). In Tables 1-24a through 1-24g and Tables 1-25a through 1-25g, gas use is for the furnace and DHW only; electricity use is for space cooling equipment and all other appliances (except DHW); and HVAC fan electricity is zero, as specified in Section 1.2.1.15. 
Table 1-24a. Case L200EX-C1H Reference Utility Energy Use Data

(Approximate input selection targeted for increased base-case space heating energy consumption)

\begin{tabular}{|c|c|c|}
\hline $\begin{array}{l}\text { Month } \\
\text { (Note 1) }\end{array}$ & $\begin{array}{c}\text { Total Gas Use } \\
\text { (million Btu) (Note 2) }\end{array}$ & $\begin{array}{l}\text { Total Electricity Use } \\
\text { (kWh) (Notes 3, 4) }\end{array}$ \\
\hline January & 29.60 & 569.6 \\
\hline February & 22.14 & 514.5 \\
\hline March & 21.76 & 569.6 \\
\hline April & 14.12 & 551.3 \\
\hline May & 5.88 & 569.6 \\
\hline June & 1.38 & 551.3 \\
\hline July & 1.43 & 569.6 \\
\hline August & 1.43 & 569.6 \\
\hline September & 2.44 & 551.3 \\
\hline October & 9.75 & 569.6 \\
\hline November & 19.23 & 551.3 \\
\hline December & 26.34 & 569.6 \\
\hline January & 29.60 & 569.6 \\
\hline \multicolumn{3}{|c|}{ Note 1: For first day of month to last day of month; 28 days in February. } \\
\hline \multicolumn{3}{|c|}{ Note 2: Gas use is for the furnace and DHW only. } \\
\hline \multicolumn{3}{|c|}{$\begin{array}{l}\text { Note 3: Electricity use is for all non-space-heating system appliances except DHW. HVAC } \\
\text { fan electricity is not included (= } 0 \text { as specified in Section 1.2.1.15). }\end{array}$} \\
\hline \multicolumn{3}{|c|}{ Note 4: $0.2930711 \mathrm{Wh}=1 \mathrm{Btu}$ (ASHRAE 2005). } \\
\hline
\end{tabular}

Table 1-24b. Case L200EX-C2H Reference Utility Energy Use Data

(Approximate input selection targeted for decreased base-case space heating energy consumption)

\begin{tabular}{|c|c|c|}
\hline $\begin{array}{l}\text { Month } \\
\text { (Note 1) }\end{array}$ & $\begin{array}{c}\text { Total Gas Use } \\
\text { (million Btu) (Note 2) }\end{array}$ & $\begin{array}{l}\text { Total Electricity Use } \\
\text { (kWh) (Notes 3, 4) }\end{array}$ \\
\hline January & 18.93 & 745.8 \\
\hline February & 13.86 & 673.7 \\
\hline March & 13.50 & 745.8 \\
\hline April & 7.55 & 721.8 \\
\hline May & 2.03 & 745.8 \\
\hline June & 1.97 & 721.8 \\
\hline July & 2.03 & 745.8 \\
\hline August & 2.03 & 745.8 \\
\hline September & 1.97 & 721.8 \\
\hline October & 2.98 & 745.8 \\
\hline November & 11.65 & 721.8 \\
\hline December & 16.55 & 745.8 \\
\hline January & 18.93 & 745.8 \\
\hline \multicolumn{3}{|c|}{ Note 1: For first day of month to last day of month; 28 days in February. } \\
\hline \multicolumn{3}{|c|}{ Note 2: Gas use is for the furnace and DHW only. } \\
\hline \multicolumn{3}{|c|}{$\begin{array}{l}\text { Note 3: Electricity use is for all non-space-heating system appliances except DHW } \\
\text { HVAC fan electricity is not included (= } 0 \text { as specified in Section 1.2.1.15). }\end{array}$} \\
\hline \multicolumn{3}{|c|}{ Note 4: $0.2930711 \mathrm{Wh}=1 \mathrm{Btu}$ (ASHRAE 2005). } \\
\hline
\end{tabular}


Table 1-24c. Case L200EX-C3H Reference Utility Energy Use Data

(Fully random approximate input selection, selected for near-nominal base-case space heating energy consumption)

\begin{tabular}{|c|c|c|}
\hline $\begin{array}{l}\text { Month } \\
\text { (Note 1) }\end{array}$ & $\begin{array}{c}\text { Total Gas Use } \\
\text { (million Btu) (Note 2) }\end{array}$ & $\begin{array}{l}\text { Total Electricity Use } \\
\text { (kWh) (Notes 3, 4) }\end{array}$ \\
\hline January & 24.24 & 381.0 \\
\hline February & 18.18 & 344.1 \\
\hline March & 17.92 & 381.0 \\
\hline April & 11.76 & 368.7 \\
\hline May & 3.61 & 381.0 \\
\hline June & 1.88 & 368.7 \\
\hline July & 1.94 & 381.0 \\
\hline August & 1.94 & 381.0 \\
\hline September & 1.88 & 368.7 \\
\hline October & 6.88 & 381.0 \\
\hline November & 15.87 & 368.7 \\
\hline December & 21.66 & 381.0 \\
\hline January & 24.24 & 381.0 \\
\hline \multicolumn{3}{|c|}{ Note 1: For first day of month to last day of month; 28 days in February. } \\
\hline \multicolumn{3}{|c|}{ Note 2: Gas use is for the furnace and DHW only. } \\
\hline \multicolumn{3}{|c|}{$\begin{array}{l}\text { Note 3: Electricity use is for all non-space-heating system appliances except DHW } \\
\text { HVAC fan electricity is not included (= } 0 \text { as specified in Section 1.2.1.15). }\end{array}$} \\
\hline \multicolumn{3}{|c|}{ Note 4: 0.2930711 Wh = 1 Btu (ASHRAE 2005). } \\
\hline
\end{tabular}

Table 1-24d. Case L200EX-C4H Reference Utility Energy Use Data (Fully random approximate input selection, selected for high base-case space heating energy consumption)

\begin{tabular}{|c|c|c|}
\hline $\begin{array}{c}\text { Month } \\
\text { (Note 1) }\end{array}$ & $\begin{array}{c}\text { Total Gas Use } \\
\text { (million Btu) (Note 2) }\end{array}$ & $\begin{array}{c}\text { Total Electricity Use } \\
\text { (kWh) (Notes 3, 4) }\end{array}$ \\
\hline January & 28.87 & 318.8 \\
\hline February & 21.77 & 288.0 \\
\hline March & 21.51 & 318.8 \\
\hline April & 14.30 & 308.6 \\
\hline May & 6.97 & 318.8 \\
\hline June & 2.19 & 308.6 \\
\hline July & 2.26 & 318.8 \\
\hline August & 2.26 & 318.8 \\
\hline September & 3.56 & 308.6 \\
\hline October & 10.23 & 318.8 \\
\hline November & 19.13 & 308.6 \\
\hline \multicolumn{2}{|c|}{25.84} & 318.8 \\
\hline \multicolumn{2}{|c|}{ December } & 318.8 \\
\hline \hline January & Note 1: For first day of month to last day of month; 28 days in February. \\
\hline Note 2: Gas use is for the furnace and DHW only. \\
\hline $\begin{array}{l}\text { Note 3: Electricity use is for all non-space-heating system appliances except DHW. } \\
\text { HVAC fan electricity is not included (= 0 as specified in Section 1.2.1.15). }\end{array}$ \\
\hline Note 4: 0.2930711 Wh = 1 Btu (ASHRAE 2005). \\
\hline
\end{tabular}


Table 1-24e. Case L200EX-C5H Reference Utility Energy Use Data

(Fully random approximate input selection, selected for low base-case space heating energy consumption)

\begin{tabular}{|c|c|c|}
\hline $\begin{array}{l}\text { Month } \\
\text { (Note 1) }\end{array}$ & $\begin{array}{c}\text { Total Gas Use } \\
\text { (million Btu) (Note 2) }\end{array}$ & $\begin{array}{l}\text { Total Electricity Use } \\
\text { (kWh) (Notes 3, 4) }\end{array}$ \\
\hline January & 18.01 & 749.5 \\
\hline February & 13.04 & 676.9 \\
\hline March & 12.58 & 749.5 \\
\hline April & 7.73 & 725.3 \\
\hline May & 2.25 & 749.5 \\
\hline June & 1.99 & 725.3 \\
\hline July & 2.06 & 749.5 \\
\hline August & 2.06 & 749.5 \\
\hline September & 1.99 & 725.3 \\
\hline October & 3.60 & 749.5 \\
\hline November & 10.78 & 725.3 \\
\hline December & 15.61 & 749.5 \\
\hline January & 18.01 & 749.5 \\
\hline \multicolumn{3}{|c|}{ Note 1: For first day of month to last day of month; 28 days in February. } \\
\hline \multicolumn{3}{|c|}{ Note 2: Gas use is for the furnace and DHW only. } \\
\hline \multicolumn{3}{|c|}{$\begin{array}{l}\text { Note 3: Electricity use is for all non-space-heating system appliances except DHW } \\
\text { HVAC fan electricity is not included (= } 0 \text { as specified in Section 1.2.1.15). }\end{array}$} \\
\hline \multicolumn{3}{|c|}{ Note 4: $0.2930711 \mathrm{Wh}=1 \mathrm{Btu}$ (ASHRAE 2005). } \\
\hline
\end{tabular}

Table 1-24f. Case L200EX-C6H Reference Utility Energy Use Data

(Fully random approximate input selection, selected for mid-high base-case space heating energy consumption)

\begin{tabular}{|c|c|c|}
\hline $\begin{array}{c}\text { Month } \\
\text { (Note 1) }\end{array}$ & $\begin{array}{c}\text { Total Gas Use } \\
\text { (million Btu) (Note 2) }\end{array}$ & $\begin{array}{c}\text { Total Electricity Use } \\
\text { (kWh) (Notes 3, 4) }\end{array}$ \\
\hline January & 25.68 & 605.6 \\
\hline February & 19.20 & 547.0 \\
\hline March & 18.98 & 605.6 \\
\hline April & 12.33 & 586.1 \\
\hline May & 4.72 & 605.6 \\
\hline June & 1.70 & 586.1 \\
\hline July & 1.75 & 605.6 \\
\hline August & 1.75 & 605.6 \\
\hline September & 1.70 & 586.1 \\
\hline October & 8.08 & 605.6 \\
\hline November & 16.66 & 586.1 \\
\hline \multicolumn{2}{|c|}{22.85} & 605.6 \\
\hline \multicolumn{2}{|c|}{25.68} \\
\hline \multicolumn{2}{|c|}{ December } & Nanuary \\
\hline \hline
\end{tabular}


Table 1-24g. Case L200EX-C7H Reference Utility Energy Use Data

(Fully random approximate input selection, selected for mid-low base-case space heating energy consumption)

\begin{tabular}{|c|c|c|}
\hline $\begin{array}{l}\text { Month } \\
\text { (Note 1) }\end{array}$ & $\begin{array}{c}\text { Total Gas Use } \\
\text { (million Btu) (Note 2) }\end{array}$ & $\begin{array}{l}\text { Total Electricity Use } \\
\text { (kWh) (Notes 3, 4) }\end{array}$ \\
\hline January & 20.24 & 780.3 \\
\hline February & 14.83 & 704.8 \\
\hline March & 14.49 & 780.3 \\
\hline April & 9.02 & 755.1 \\
\hline May & 3.49 & 780.3 \\
\hline June & 1.52 & 755.1 \\
\hline July & 1.57 & 780.3 \\
\hline August & 1.57 & 780.3 \\
\hline September & 1.52 & 755.1 \\
\hline October & 5.38 & 780.3 \\
\hline November & 12.49 & 755.1 \\
\hline December & 17.70 & 780.3 \\
\hline January & 20.24 & 780.3 \\
\hline \multicolumn{3}{|c|}{ Note 1: For first day of month to last day of month; 28 days in February. } \\
\hline \multicolumn{3}{|c|}{ Note 2: Gas use is for the furnace and DHW only. } \\
\hline \multicolumn{3}{|c|}{$\begin{array}{l}\text { Note 3: Electricity use is for all non-space-heating system appliances except DHW } \\
\text { HVAC fan electricity is not included (= } 0 \text { as specified in Section } 1.2 .1 .15) \text {. }\end{array}$} \\
\hline \multicolumn{3}{|c|}{ Note 4: $0.2930711 \mathrm{Wh}=1 \mathrm{Btu}$ (ASHRAE 2005). } \\
\hline
\end{tabular}

Table 1-25a. Case L200EX-C1C Reference Utility Energy Use Data

(Approximate input selection targeted for increased base-case space cooling energy consumption)

\begin{tabular}{|c|c|c|}
\hline $\begin{array}{l}\text { Month } \\
\text { (Note 1) }\end{array}$ & $\begin{array}{c}\text { Total Gas Use } \\
\text { (million Btu) (Note 2) }\end{array}$ & $\begin{array}{l}\text { Total Electricity Use } \\
\text { (kWh) (Notes } 3,4)\end{array}$ \\
\hline January & 1.98 & 687.4 \\
\hline February & 1.79 & 620.9 \\
\hline March & 1.98 & 787.3 \\
\hline April & 1.92 & 1167.9 \\
\hline May & 1.98 & 1449.0 \\
\hline June & 1.92 & 2006.2 \\
\hline July & 1.98 & 2284.2 \\
\hline August & 1.98 & 2190.2 \\
\hline September & 1.92 & 1781.2 \\
\hline October & 1.98 & 1203.0 \\
\hline November & 1.92 & 665.3 \\
\hline December & 1.98 & 687.4 \\
\hline January & 1.98 & 687.4 \\
\hline \multicolumn{3}{|c|}{ Note 1: For first day of month to last day of month; 28 days in February. } \\
\hline \multicolumn{3}{|c|}{ Note 2: Gas use is for DHW only. } \\
\hline \multicolumn{3}{|c|}{$\begin{array}{l}\text { Note 3: Electricity use is for space conditioning equipment and all appliances except } \\
\text { DHW. HVAC fan electricity is not included (= } 0 \text { as specified in Section 1.2.1.15). }\end{array}$} \\
\hline \multicolumn{3}{|c|}{ Note 4: $0.2930711 \mathrm{Wh}=1 \mathrm{Btu}(\mathrm{ASHRAE} 2005)$. } \\
\hline
\end{tabular}


Table 1-25b. Case L200EX-C2C Reference Utility Energy Use Data

(Approximate input selection targeted for decreased base-case space cooling energy consumption)

\begin{tabular}{|c|c|c|}
\hline $\begin{array}{c}\text { Month } \\
\text { (Note 1) }\end{array}$ & $\begin{array}{c}\text { Total Gas Use } \\
\text { (million Btu) (Note 2) }\end{array}$ & $\begin{array}{l}\text { Total Electricity Use } \\
\text { (kWh) (Notes 3, 4) }\end{array}$ \\
\hline January & 2.67 & 322.7 \\
\hline February & 2.41 & 291.5 \\
\hline March & 2.67 & 372.0 \\
\hline April & 2.58 & 703.3 \\
\hline May & 2.67 & 920.4 \\
\hline June & 2.58 & 1368.4 \\
\hline July & 2.67 & 1584.4 \\
\hline August & 2.67 & 1505.5 \\
\hline September & 2.58 & 1191.6 \\
\hline October & 2.67 & 701.5 \\
\hline November & 2.58 & 312.3 \\
\hline December & 2.67 & 322.7 \\
\hline January & 2.67 & 322.7 \\
\hline \multicolumn{3}{|c|}{ Note 1: For first day of month to last day of month; 28 days in February. } \\
\hline \multicolumn{3}{|c|}{ Note 2: Gas use is for DHW only. } \\
\hline \multicolumn{3}{|c|}{$\begin{array}{l}\text { Note 3: Electricity use is for space conditioning equipment and all appliances excep } \\
\text { DHW. HVAC fan electricity is not included (= } 0 \text { as specified in Section 1.2.1.15). }\end{array}$} \\
\hline \multicolumn{3}{|c|}{ Note 4: $0.2930711 \mathrm{Wh}=1 \mathrm{Btu}$ (ASHRAE 2005). } \\
\hline
\end{tabular}

Table 1-25c. Case L200EX-C3C Reference Utility Energy Use Data

(Fully random approximate input selection, selected for near-nominal base-case space cooling energy consumption)

\begin{tabular}{|c|c|c|}
\hline $\begin{array}{l}\text { Month } \\
\text { (Note 1) }\end{array}$ & $\begin{array}{c}\text { Total Gas Use } \\
\text { (million Btu) (Note 2) }\end{array}$ & $\begin{array}{c}\text { Total Electricity Use } \\
\text { (kWh) (Notes 3, 4) }\end{array}$ \\
\hline January & 1.53 & 558.8 \\
\hline February & 1.39 & 504.7 \\
\hline March & 1.53 & 558.8 \\
\hline April & 1.49 & 928.4 \\
\hline May & 1.53 & 1177.1 \\
\hline June & 1.49 & 1616.5 \\
\hline July & 1.53 & 1839.4 \\
\hline August & 1.53 & 1760.6 \\
\hline September & 1.49 & 1434.0 \\
\hline October & 1.53 & 870.3 \\
\hline November & 1.49 & 540.8 \\
\hline December & 1.53 & 558.8 \\
\hline January & 1.53 & 558.8 \\
\hline \multicolumn{3}{|c|}{ Note 1: For first day of month to last day of month; 28 days in February. } \\
\hline \multirow{2}{*}{\multicolumn{3}{|c|}{$\begin{array}{l}\text { Note 2: Gas use is for DHW only. } \\
\text { Note 3: Electricity use is for space conditioning equipment and all appliances excep } \\
\text { DHW. HVAC fan electricity is not included (= } 0 \text { as specified in Section 1.2.1.15). }\end{array}$}} \\
\hline & & \\
\hline \multicolumn{3}{|c|}{ Note 4: 0.2930711 Wh = $1 \mathrm{Btu}$ (ASHRAE 2005). } \\
\hline
\end{tabular}


Table 1-25d. Case L200EX-C4C Reference Utility Energy Use Data

(Fully random approximate input selection, selected for high base-case space cooling energy consumption)

\begin{tabular}{|c|c|c|}
\hline $\begin{array}{l}\text { Month } \\
\text { (Note 1) }\end{array}$ & $\begin{array}{c}\text { Total Gas Use } \\
\text { (million Btu) (Note 2) }\end{array}$ & $\begin{array}{c}\text { Total Electricity Use } \\
\text { (kWh) (Notes } 3,4)\end{array}$ \\
\hline January & 2.15 & 654.8 \\
\hline February & 1.94 & 591.5 \\
\hline March & 2.15 & 777.2 \\
\hline April & 2.08 & 1118.8 \\
\hline May & 2.15 & 1393.3 \\
\hline June & 2.08 & 1938.2 \\
\hline July & 2.15 & 2211.2 \\
\hline August & 2.15 & 2115.6 \\
\hline September & 2.08 & 1712.4 \\
\hline October & 2.15 & 1165.9 \\
\hline November & 2.08 & 645.2 \\
\hline December & 2.15 & 654.8 \\
\hline January & 2.15 & 654.8 \\
\hline \multicolumn{3}{|c|}{ Note 1: For first day of month to last day of month; 28 days in February. } \\
\hline \multirow{2}{*}{\multicolumn{3}{|c|}{$\begin{array}{l}\text { Note 2: Gas use is for DHW only. } \\
\text { Note 3: Electricity use is for space conditioning equipment and all appliances except } \\
\text { DHW. HVAC fan electricity is not included (= } 0 \text { as specified in Section 1.2.1.15). }\end{array}$}} \\
\hline & & \\
\hline \multicolumn{3}{|c|}{ Note 4: $0.2930711 \mathrm{Wh}=1 \mathrm{Btu}$ (ASHRAE 2005). } \\
\hline
\end{tabular}

Table 1-25e. Case L200EX-C5C Reference Utility Energy Use Data

(Fully random approximate input selection, selected for low base-case space cooling energy consumption)

\begin{tabular}{|c|c|c|}
\hline $\begin{array}{l}\text { Month } \\
\text { (Note 1) }\end{array}$ & $\begin{array}{c}\text { Total Gas Use } \\
\text { (million Btu) (Note 2) }\end{array}$ & $\begin{array}{l}\text { Total Electricity Use } \\
\text { (kWh) (Notes 3, 4) }\end{array}$ \\
\hline January & 1.34 & 419.6 \\
\hline February & 1.21 & 379.0 \\
\hline March & 1.34 & 436.6 \\
\hline April & 1.30 & 639.5 \\
\hline May & 1.34 & 814.5 \\
\hline June & 1.30 & 1193.0 \\
\hline July & 1.34 & 1379.0 \\
\hline August & 1.34 & 1315.1 \\
\hline September & 1.30 & 1049.4 \\
\hline October & 1.34 & 674.3 \\
\hline November & 1.30 & 406.0 \\
\hline December & 1.34 & 419.6 \\
\hline January & 1.34 & 419.6 \\
\hline \multicolumn{3}{|c|}{ Note 1: For first day of month to last day of month; 28 days in February. } \\
\hline \multirow{2}{*}{\multicolumn{3}{|c|}{$\begin{array}{l}\text { Note 2: Gas use is for DHW only. } \\
\text { Note 3: Electricity use is for space conditioning equipment and all appliances except } \\
\text { DHW. HVAC fan electricity is not included (= } 0 \text { as specified in Section 1.2.1.15). }\end{array}$}} \\
\hline & & \\
\hline \multicolumn{3}{|c|}{ Note 4: $0.2930711 \mathrm{Wh}=1 \mathrm{Btu}$ (ASHRAE 2005). } \\
\hline
\end{tabular}


Table 1-25f. Case L200EX-C6C Reference Utility Energy Use Data

(Fully random approximate input selection, selected for mid-high base-case space cooling energy consumption)

\begin{tabular}{|c|c|c|}
\hline $\begin{array}{l}\text { Month } \\
\text { (Note 1) }\end{array}$ & $\begin{array}{c}\text { Total Gas Use } \\
\text { (million Btu) (Note 2) }\end{array}$ & $\begin{array}{l}\text { Total Electricity Use } \\
\text { (kWh) (Notes 3, 4) }\end{array}$ \\
\hline January & 1.98 & 764.0 \\
\hline February & 1.79 & 690.1 \\
\hline March & 1.98 & 764.0 \\
\hline April & 1.92 & 1144.2 \\
\hline May & 1.98 & 1427.7 \\
\hline June & 1.92 & 1944.3 \\
\hline July & 1.98 & 2208.7 \\
\hline August & 1.98 & 2118.4 \\
\hline September & 1.92 & 1737.4 \\
\hline October & 1.98 & 1154.5 \\
\hline November & 1.92 & 739.4 \\
\hline December & 1.98 & 764.0 \\
\hline January & 1.98 & 764.0 \\
\hline \multicolumn{3}{|c|}{ Note 1: For first day of month to last day of month; 28 days in February. } \\
\hline \multicolumn{3}{|c|}{ Note 2: Gas use is for DHW only. } \\
\hline \multicolumn{3}{|c|}{$\begin{array}{l}\text { Note 3: Electricity use is for space conditioning equipment and all appliances excep } \\
\text { DHW. HVAC fan electricity is not included (=0 as specified in Section 1.2.1.15). }\end{array}$} \\
\hline \multicolumn{3}{|c|}{ Note 4: $0.2930711 \mathrm{Wh}=1 \mathrm{Btu}$ (ASHRAE 2005). } \\
\hline
\end{tabular}

Table 1-25g. Case L200EX-C7C Reference Utility Energy Use Data

(Fully random approximate input selection, selected for mid-low base-case space cooling energy consumption)

\begin{tabular}{|c|c|c|}
\hline $\begin{array}{l}\text { Month } \\
\text { (Note 1) }\end{array}$ & $\begin{array}{c}\text { Total Gas Use } \\
\text { (million Btu) (Note 2) }\end{array}$ & $\begin{array}{l}\text { Total Electricity Use } \\
\text { (kWh) (Notes 3, 4) }\end{array}$ \\
\hline January & 1.71 & 851.3 \\
\hline February & 1.54 & 768.9 \\
\hline March & 1.71 & 851.3 \\
\hline April & 1.65 & 1106.9 \\
\hline May & 1.71 & 1368.9 \\
\hline June & 1.65 & 1783.5 \\
\hline July & 1.71 & 2008.0 \\
\hline August & 1.71 & 1935.5 \\
\hline September & 1.65 & 1624.0 \\
\hline October & 1.71 & 1117.6 \\
\hline November & 1.65 & 823.9 \\
\hline December & 1.71 & 851.3 \\
\hline January & 1.71 & 851.3 \\
\hline \multicolumn{3}{|c|}{ Note 1: For first day of month to last day of month; 28 days in February. } \\
\hline \multicolumn{3}{|c|}{ Note 2: Gas use is for DHW only. } \\
\hline \multicolumn{3}{|c|}{$\begin{array}{l}\text { Note 3: Electricity use is for space conditioning equipment and all appliances except } \\
\text { DHW. HVAC fan electricity is not included (= } 0 \text { as specified in Section 1.2.1.15). }\end{array}$} \\
\hline \multicolumn{3}{|c|}{ Note 4: $0.2930711 \mathrm{Wh}=1 \mathrm{Btu}(\mathrm{ASHRAE} 2005)}$. \\
\hline
\end{tabular}




\subsubsection{Topical Details}

\subsection{Infiltration}

The approximate input ranges shown in Tables 1-2, 1-8a, 1-8b (containing infiltration input data) are meant to include all factors that would account for uncertainty in the resulting energy load, including the uncertainties associated with blower door measurements caused by user error, instrument calibration error, measurement repeatability, outdoor wind speed, etc., along with other modeling uncertainties such as crack type, leak location, etc. Although, it may be more realistic to exclude uncertainties not related directly to one-point $\mathrm{CFM}_{50}$ (cubic feet per minute at 50 Pascals) measurements from the $\mathrm{CFM}_{50}$ approximate input range, it is simpler to specify consistent approximate input ranges for all inputs, as different tested programs may require different inputs. Therefore, resulting approximate input range as percent variation from nominal value for effective leakage area at $4 \mathrm{~Pa}$ is the same as for $\mathrm{CFM}_{50}$ (see Table 1-8a).

\subsection{Internal Loads}

\subsection{Sensible Loads}

Approximate input ranges for daily total sensible internal loads are specified in Table 1-9a. Normalized sensible load hourly profile fractions for the conditioned zone are specified in Table 1-9b; the hourly fractions apply for all days of the year as given. Approximate input ranges are given for the daily sensible internal loads disaggregated for occupants, electricity, and gas. For developing reference utility billing data and other reference simulation results (see Section 1.3.1.2), a different randomly selected explicit input for daily total value is chosen for each of the three categories within the minimum and maximum values listed in Table 1-9a. The hourly internal sensible gains are then calculated by multiplying the randomly selected daily totals by the schedules listed in Table 1-9b.

\subsection{Latent Loads}

As with the building physics tests of Section 1.2, latent loads are ignored (see Section 1.2.1.8.2). For developing reference simulation results, latent loads were not included, as they have no effect on the results.

\subsection{Fractions of Base Load Usages to Internal Gains}

Only a fraction of the non-HVAC energy from electricity and gas used in a home is converted to sensible internal gains. To generate synthetic utility bills, percentages of non-HVAC gas energy and electric energy converted to sensible loads must be assumed. The following approximate input ranges are used for conversion of non-HVAC energy use to sensible internal gains:

- $\quad \mathbf{6 0} \%-\mathbf{9 0 \%}$ of the non-HVAC energy for electric appliances and lights

- $\mathbf{2 0} \%-\mathbf{3 5 \%}$ of the non-HVAC energy for gas DHW.

These values were developed in consultation with the BESTEST-EX Working Group (2009). Further background discussion is included in Appendix B. Resulting ranges of non-HVAC energy usage based on these fractions are included in Table 1-9a.

Synthetic reference utility energy use data (provided in Section 1.3.1.2) integrate internal gains using the following steps:

1. Randomly select explicit conversion factors for electricity (X\%) and gas (Y\%) from within the approximate input ranges described above. 
2. Perform the conversions:

a. Convert the sensible internal gains due to non-HVAC electric appliances and lights (divide by $\mathrm{X} \% / 100$ ) to obtain base load electricity consumption.

b. Convert the sensible internal gains due to non-HVAC gas appliances (DHW) (divide by $\mathrm{Y} \% / 100$ ) to obtain base load (DHW) gas consumption.

3. Each month:

a. Add the non-HVAC electricity consumption to the monthly HVAC electricity consumption.

b. Add the non-HVAC gas consumption to the monthly HVAC gas consumption.

4. Use units of $\mathrm{kWh}$ for metered electricity consumption and million $\left(10^{6}\right)$ Btu for metered natural gas consumption.

\subsection{Opaque Exterior Surface Radiative Properties}

The approximate input range for exterior surface solar absorptance is $0.5-0.8$ for all opaque exterior surfaces except window frames. Window frames remain at 0.6 . All other opaque surface radiative properties have explicit inputs as shown in Table 1-2.

For the reference simulations, the same randomly selected exterior solar absorptance value applies to all wall and roof exterior surfaces for a given base-case scenario.

\subsection{Thermostat Control Strategies}

Seasonal thermostat control settings are shown for heating and cooling climates in Sections 1.3.1.3.4.1 and 1.3.1.3.4.2, respectively. The heating and cooling season start/stop dates are intentionally not given to the user. For developing the reference utility data (see Section 1.3.1.2), the start/stop dates are randomly selected from within an approximate input range based on the period when a given fraction of full-year space conditioning load (heating or cooling load, as appropriate) would occur for the randomly selected base-case explicit inputs (including the randomly selected thermostat set point), as follows:

- L200EX-C1 (targeted high space conditioning): 95\%-99\% of full-year load

- L200EX-C2 (targeted low space conditioning): $90 \%-95 \%$ of full-year load

- L200EX-C3 through C7 (fully random selection): 90\%-99\% of full-year load.

\subsection{Colorad.TM2}

For Colorad.TM2 weather data (heating only)

During heating season (season start/stop dates intentionally not given):

$$
\text { HEAT }=\text { ON IF TEMP }<\mathbf{T}_{\mathbf{h t g}} ; \mathrm{COOL}=\text { OFF }
$$

During non-heating season (season start/stop dates intentionally not given):

$$
\mathrm{HEAT}=\mathrm{OFF} ; \mathrm{COOL}=\mathrm{OFF} \text {. }
$$

Where:

- “TEMP” refers to conditioned zone-air temperature

- The approximate input range for $T_{h t g}$ is $60^{\circ}-75^{\circ} \mathrm{F}$.

\subsection{Lasvega.TM2}

For Lasvega.TM2 weather data (cooling only)

During cooling season (season start/stop dates intentionally not given):

$$
\mathrm{COOL}=\mathrm{ON} \text { IF TEMP }>\mathbf{T}_{\text {clg }} ; \text { HEAT }=\text { OFF }
$$


During non-cooling season (season start/stop dates intentionally not given):

$$
\mathrm{COOL}=\mathrm{OFF} ; \mathrm{HEAT}=\mathrm{OFF} \text {. }
$$

Where:

- "TEMP" refers to conditioned zone-air temperature.

- The approximate input range for $T_{\text {clg }}$ is $71^{\circ}-86^{\circ} \mathrm{F}$.

\subsection{Equipment Characteristics}

EFFECTIVE HEATING EFFICIENCY $=\mathbf{E}_{\mathbf{h t g}}$

EFFECTIVE COOLING COEFFICIENT OF PERFORMANCE $=\mathbf{C O P}_{\mathbf{c l g}}$

Where:

- The approximate input range for $E_{h t g}$ is $60 \%-80 \%$.

- The approximate input range for $\mathrm{COP}_{\mathrm{clg}}$ is 2.5-3.5.

For generating reference utility energy use data, randomly selected heating efficiencies and cooling COPs are modeled as constant: independent of part loading, indoor dry-bulb temperature and humidity ratio, outdoor dry-bulb temperature and humidity ratio, and other conditions.

\subsubsection{Calibrated Energy Savings Retrofit Test Cases}

This section describes revisions to Case L200EX-C required to model the calibrated energy savings retrofit test cases.

In these test cases, the modeling physics of the retrofit technology as applied to the calibrated base-case model are precisely known. Use the calibrated inputs developed for each Case L200EX-C base-case scenario as the basis for the retrofit cases. There are seven base-case scenarios for each climate (14 total for heating and cooling) with six energy-savings cases in the heating climate and seven energy-savings cases in the cooling climate within each scenario. This results in a total of $(7 \times 7)+(7 \times 8)=105$ total calibrated energy-savings case results sets, including the base case results sets. These are labeled as LnnnEX-C1H through LnnnEX-C7H for cases with space heating, and LnnnEX-C1C through LnnnEXC7C for cases with space cooling.

For convenience, except where noted, figures and tables used for the building physics (EX-P suffixed) retrofit cases of Section 1.2.2 are applied for developing the calibrated retrofit cases. Nominal inputs included (in non-bold font) in Section 1.2.2 tables called out in Section 1.3.2 are for reference only. Do not apply the nominal (non-bold font) values given in those tables if they were varied as part of the calibration process for Case L200EX-C. Changes to the calibrated base-case model to be applied as explicit inputs, or as explicit changes to calibrated inputs, in the calibrated energy savings ("-C") retrofit cases are highlighted with bold font in the tables.

Where applicable, summary figures and tables are listed first; supplementary tables are listed afterward.

The retrofits are done as relative changes to the calibrated base-case inputs, except for low-e windows (Case L250EX-C) and cool roof (Case L265EX-C). For example, if in Case L200EX-C calibration yielded a pre-retrofit "air-air composite" ceiling/attic/roof R-value $=9$ (versus nominal R-13.7), apply the blown cellulose retrofit explicitly as indicated to the calibrated (R-9) ceiling/attic/roof model. In this case, resulting composite R-values given in the material description table for the building physics retrofit case (L220EX-P) do not apply for the calibrated energy savings retrofit case (L220EX-C). 


\subsubsection{Case L210EX-C: Air-Seal Retrofit}

Case L210EX-C is exactly as Case L200EX-C, except apply a decrease to the effective leakage area at 4 $\mathrm{Pa}$ (ELA4) of $100 \mathrm{in}^{2}$ to the calibrated base-case input files for the base-case scenarios (L200EX-C1 through L200EX-C7); i.e., for each scenario (“-Cn”):

$$
\mathrm{ELA}_{\mathrm{L} 210 \mathrm{EX}-\mathrm{Cn}}=\mathrm{ELA}_{\mathrm{L} 200 \mathrm{EX}-\mathrm{Cn}}-100 \quad \text { (in. }^{2} \text { ) }
$$

Where:

$$
\begin{aligned}
& \mathrm{ELA}_{\mathrm{L} 210 \mathrm{EX}-\mathrm{Cn}}=\text { resulting effective leakage area for Case L210EX-Cn } \\
& \text { ELA4 }_{\text {L200EX-Cn }}=\quad \text { calibrated base-case effective leakage area for Case L200EX-Cn, where }
\end{aligned}
$$

Equivalent decreases to other relevant equivalent inputs that may be used by various programs are

\begin{tabular}{|c|c|c|c|}
\hline Input (Note 1) & \multicolumn{3}{|c|}{ Value Decrease } \\
\hline CFM at $50 \mathrm{~Pa}$ (Colorado Springs) (Note 2) & \multicolumn{3}{|c|}{2038} \\
\hline CFM at $50 \mathrm{~Pa}$ (Las Vegas) (Note 2) & \multicolumn{3}{|c|}{1892} \\
\hline $\mathrm{A} \mathrm{C} \mathrm{H}$ at 50 Pa (Colorado Springs) (Note 2) & \\
\hline $\mathrm{ACH}$ at $50 \mathrm{~Pa}$ (Las Vegas) (Note 2). & \multicolumn{3}{|c|}{9.22} \\
\hline Équivalent Leakage Ärea at 50 Pa, in ${ }^{2}$ (Note 3) & \multicolumn{3}{|c|}{146.1} \\
\hline Effective Leakage Area at $4 \mathrm{~Pa}$, in ${ }^{2}$ (Note 3) & \multicolumn{3}{|c|}{100.0} \\
\hline Equivalent Constant Seasonal Decrease: ACH, CFM, UAinfl & \multirow{2}{*}{\multicolumn{3}{|c|}{$\begin{array}{c}\text { UAinfl } \\
\text { Btu/(h-F) } \\
\text { (Note 5) }\end{array}$}} \\
\hline Colorado Springs & & & \\
\hline Programs with auto-altitude adjustment (Note 4) & 0.389 & 79.8 & \multirow[t]{2}{*}{68.5} \\
\hline Programs with site fixed at sea level (Notes 4,6$)$ & 0.309 & 63.4 & \\
\hline \multicolumn{4}{|l|}{ Las Vegas } \\
\hline Programs with auto-altitude adjustment (Note 4) & 0.251 & 51.4 & \multirow[t]{2}{*}{51.2} \\
\hline Programs with site fixed at sea level (Notes 4,6$)$ & 0.231 & 47.4 & \\
\hline \multicolumn{4}{|c|}{$\begin{array}{l}\text { Note 1: Decrease the calibrated base-case value(s) appropriate for your program by the amount shown. } \\
\text { Note 2: Volumetric flow rates are different for each climate. } \\
\text { Note 3: Used for ASHRAE Residential Air Leakage model (2005 ASHRAE Handbook of Fundamentals, pp. } 27.12-27.13,27.21 \text { ); model is based on } \\
\text { Sherman-Grimsrud (1980), assuming highly sheltered building (Shelter Class 5) in rural terrain. See Appendix D for supporting details. } \\
\text { Note 4: This input is for programs that do not use more detailed methods. Given value decreases are based on } \\
\text { application of ASHRAE Residential Air-Leakage model in EnergyPlus for appropriate space conditioning season based on } \\
\text { given input decrease of effective leakage area at 4Pa, using stack coefficient and wind coefficient given previously (see Table 1-8a). } \\
\text { Note 5: Infiltration UA = (infiltration mass flow) } \times \text { (specific heat). Assumes air properties: specific heat = } 0.240 \text { Btu/(lb-F); density = } 0.075 \mathrm{lb} / \mathrm{ft}^{3} \text { at } \\
\text { sea level, adjusted for altitude per HERS BESTEST Appendix B. } \\
\text { Note 6: HERS BESTEST Appendix B describes the algorithm used for adjusting infiltration rates if the software } \\
\text { being tested does not account for variation of air density with altitude (i.e., site fixed at sea level). }\end{array}$} \\
\hline
\end{tabular}
given in Table 1-26.

This retrofit will yield results labeled L210EX-C1H through L210EX-C7H and L210EX-C1C through L210EX-C7C, corresponding to each calibrated base-case scenario.

Table 1-26. Conditioned Zone Equivalent Input Decrease for Infiltration Models - Case L210EX-C 


\subsubsection{Case L220EX-C: Attic Insulation Retrofit}

Case L220EX-C is exactly as Case L200EX-C, except apply the blown cellulose prescribed for Case L220EX-P (see Tables 1-16a and 1-16b, Section 1.2.2.2) to the calibrated base-case input files for each of the base-case scenarios. Apply only the material properties for 'blown cellulose' and for the 'Joists $2 \times 6$ 24" O.C.' using 5.5 in. thickness; do NOT change any other inputs to match those indicated in Tables 1$16 \mathrm{a}, 1-16 \mathrm{~b}$, or $1-15$, that may have been varied during the base-case calibrations. This will yield results labeled L220EX-C1H through L220EX-C7H and L220EX-C1C through L220EX-C7C, corresponding to each calibrated base-case scenario.

This retrofit is a relative change to the base case; for example (hypothetically) if in Case L200EX-C, calibration yielded a pre-retrofit "air-air composite" ceiling/attic/roof R-value $=11$ (versus nominal R13.7), apply the blown cellulose explicitly as indicated (2.0 in. over fiberglass batt and an additional 6.0 in. over both insulated and framed sections) to the calibrated (hypothetical R-11) model. Model the full 5.5 in. joist thickness in this retrofit. Depending on how the model was calibrated, total cumulative material thicknesses for the framed and insulated sections may be different. This also implies that the composite R-values for the building physics retrofit case given for the ceiling in Table 1-16a and for the ceiling/attic/roof assemblies in Tables 1-16b and 1-15 do not apply.

\subsubsection{Case L225EX-C: Wall Insulation Retrofit}

Case L225EX-C is exactly as Case L200EX-C, except replace the R-1.01 air gap of Table 1-4 with R-13 (optimally installed) blown cellulose (see Figure 1-10). Apply only the material properties for "blown cellulose" given in Table 1-18 (see Case L225EX-P, Section 1.2.2.3) to the calibrated base-case input files for each base-case scenario (L200EX-C1 through L200EX-C7). Do NOT change any other inputs to match those indicated in Table 1-18 or Table 1-4 that may have been varied during the base-case calibrations. This will yield results labeled L225EX-C1H through L225EX-C7H and L225EX-C1C through L225EX-C7C, corresponding to each calibrated base-case scenario.

\subsubsection{Case L240EX-C: Programmable Thermostat Retrofit}

Case L240EX-C is exactly as Case L200EX-C, except for changes described in Sections 1.3.2.4.1 and 1.3.2.4.2. Apply the changes to the calibrated base-case input files for each base-case scenario. This will yield results labeled L240EX-C1H through L240EX-C7H and L240EX-C1C through L240EX-C7C, corresponding to each calibrated base-case scenario.

\subsection{Colorad.TM2}

For Colorad.TM2 weather data (heating only) thermostat setback is applied on all nights during the heating season from 10:00 p.m. to 6:00 a.m. as shown below.

During heating season:

$$
\begin{aligned}
& \text { 10:00 p.m. }-6: 00 \text { a.m.: HEAT }=\text { ON IF TEMP }<\mathbf{T}_{\mathbf{h t g}}-\mathbf{6}^{\circ} \mathbf{F} ; \mathrm{COOL}=\mathrm{OFF} \\
& \text { 6:00 a.m. }-10: 00 \text { p.m.: HEAT }=\text { ON IF TEMP }<\mathbf{T}_{\mathbf{h t g}} ; \mathrm{COOL}=\mathrm{OFF}
\end{aligned}
$$

During non-heating season:

$$
\mathrm{HEAT}=\mathrm{OFF} ; \mathrm{COOL}=\mathrm{OFF} \text {. }
$$

Where:

- "TEMP" refers to conditioned zone-air temperature.

- $\quad \mathbf{T}_{\mathrm{htg}}$ is as determined during the calibration phase of Case L200EX-C (see Section 1.3.1.2).

- The heating season start/stop dates are as determined during the calibration phase of Case L200EX-C (see Section 1.3.1.2). 


\subsection{Lasvega.TM2}

For Lasvega.TM2 weather data (cooling only) thermostat setup is applied on all days from 8:00 a.m. to 5:00 p.m. as shown below.

During cooling season:

$$
\begin{aligned}
& \text { 8:00 a.m. }-5: 00 \text { p.m.: COOL }=\text { ON IF TEMP }>\mathbf{T}_{\text {clg }}+\mathbf{6}^{\circ} \mathbf{F} ; \text { HEAT }=\text { OFF } \\
& \text { 5:00 p.m. }-8: 00 \text { a.m.: COOL }=\text { ON IF TEMP }>\mathbf{T}_{\text {clg }} ; \text { HEAT }=\text { OFF }
\end{aligned}
$$

During non-cooling season:

$$
\mathrm{COOL}=\mathrm{OFF} ; \mathrm{HEAT}=\mathrm{OFF} \text {. }
$$

Where:

- "TEMP" refers to conditioned zone-air temperature.

- $\mathbf{T}_{\mathrm{clg}}$ is as determined during the calibration phase of Case L200EX-C (see Section 1.3.1.2).

- The cooling season start/stop dates are as determined during the calibration phase of Case L200EX-C (see Section 1.3.1.2).

\subsubsection{Case L250EX-C: Low-Emissivity Window Retrofit}

Case L250EX-C is exactly as Case L200EX-C, except that all single-pane windows are replaced with double-pane low-emissivity (low-e) windows with wood frames and insulated spacers, as specified for Case L250EX-P (see Section 1.2.2.5). This will yield results labeled L250EX-C1H through L250EX-C7H and L250EX-C1C through L250EX-C7C, corresponding to each calibrated base-case scenario.

As there are no approximate input ranges for the base-case single-pane window, the same single-pane window inputs are applied in the reference simulations for Case L200EX-C as were applied in Case L200EX-P. This test case may then be thought of as a "very absolute" retrofit, where retrofit of the singlepane window system with the low-e window system is applied exactly as in Case L250EX-P, except that other conditions (thermostat settings, thermal conduction through other envelope surfaces, equipment efficiency, etc.) may vary in the Case L200EX-C calibration.

\subsubsection{Case L265EX-C: Low Solar Absorptance Roof (Cool Roof)}

Case L265EX-C is exactly as Case L200EX-C, except that exterior shortwave (visible and UV) absorptance $\left(\alpha_{\text {ext }}\right)$ is 0.2 for the roof only. This case is to be done by applying only space-cooling-only thermostat settings (see Section 1.3.1.3.4.2) using the Lasvega.TM2 weather data. This will yield results labeled L265EX-C1C through L265EX-C7C, corresponding to each calibrated base-case scenario.

This test case may be thought of as an "absolute" retrofit, where energy savings for the fixed value $\left(\alpha_{\text {ext }}=0.2\right)$ cool roof retrofit are calculated based on the calibrated opaque surface solar absorptance selected from $0.5 \leq \alpha_{\mathrm{ext}} \leq 0.8$ during the calibration phase of Case L200EX-C (see Section 1.3.1.3.3).

\subsubsection{Case L3O0EX-C: Combined Retrofits}

Apply the changes specified for Case L300EX-C (see Sections 1.3.2.7.1 and 1.3.2.7.2) to the calibrated base-case input files for each base-case scenario. This will yield results labeled L300EX-C1H through L300EX-C7H and L300EX-C1C through L300EX-C7C, corresponding to each calibrated base-case scenario.

Case L300EX-C is exactly as Case L200EX-C, except for the following changes. 


\subsection{Case L300EX-CH}

For the heating climate (Colorad.TM2 weather) include the:

- Air-seal retrofit as described in Section 1.3.2.1

- Attic insulation retrofit as described in Section 1.3.2.2

- Wall insulation retrofit as described in Section 1.3.2.3

- Programmable thermostat retrofit as described in Section 1.3.2.4

- Low-e window retrofit as described in Section 1.3.2.5.

\subsection{Case L300EX-CC}

For the cooling climate (Lasvega.TM2 weather) include:

- $\quad$ All the retrofits listed in Section 1.3.2.7.1

- The low solar-absorptance roof retrofit as described in Section 1.3.2.6. 


\section{References}

ANSI/ASHRAE Standard 140-2007. (2007). Standard Method of Test for the Evaluation of Building Energy Analysis Computer Programs. Atlanta, GA: American Society of Heating, Refrigerating and AirConditioning Engineers. www.ashrae.org.

ANSI/ASHRAE/IESNA. (2007). ANSI/ASHRAE/IESNA Standard 90.1-2007, Energy Standard for Buildings Except Low-Rise Residential Buildings. Atlanta, GA: American Society of Heating, Refrigerating and Air-Conditioning Engineers.

ASHRAE Handbook Fundamentals. (1993). Atlanta, GA: American Society of Heating, Refrigerating and Air-Conditioning Engineers.

ASHRAE Handbook Fundamentals. (2001). Atlanta, GA: American Society of Heating, Refrigerating and Air-Conditioning Engineers.

ASHRAE Handbook Fundamentals. (2005). Atlanta, GA: American Society of Heating, Refrigerating and Air-Conditioning Engineers.

ASHRAE Handbook Fundamentals. (2009). Atlanta, GA: American Society of Heating, Refrigerating and Air-Conditioning Engineers.

BA. (2009). Building America Analysis Spreadsheet. Washington, D.C.: U.S. Department of Energy, Office of Energy Efficiency and Renewable Energy. www1.eere.energy.gov/buildings/building america/ perf analysis.html. Last accessed January 2010.

Berry, L.; Gettings, M. (1998). "Realization Rates of the National Energy Audit." Thermal Performance of the Exterior Envelopes of Buildings VII Conference. December 7-11, Clearwater Beach, FL.

BESTEST-EX Working Group. (2009-2010). Various BESTEST-EX Working Group discussions. January 2009-April 2010. Meeting notes available on request.

Blasnik, M. (2009). M. Blasnik and Associates. Personal communication with J. Neymark, J. Neymark \& Associates, Wheat Ridge, CO, March 27, 2009.

Blasnik, M. (2010). M. Blasnik and Associates. Personal communication with J. Neymark, J. Neymark \& Associates, Wheat Ridge, CO, May 5, 2010.

BLAST User Reference, Volume 1 and Volume 2. (1991). BLAST Support Office. Urbana, IL: University of Illinois. (This program is no longer supported.)

Dalhoff, G. (1997). "An Evaluation of the Performance of the NEAT Audit for the Iowa Low-Income Weatherization Program.” 1997 Energy Evaluation Conference. Chicago, IL.

Deru, M.; Judkoff, R.; Torcellini, P. (2002). SUNREL Technical Reference Manual. NREL/BK-55030193. Golden, CO: National Renewable Energy Laboratory.

DOE. (1989). Affordable Housing Through Energy Conservation - A Guide to Designing and Constructing Energy Efficient Homes (Technical Support Document). DOE/SF/00098-H3, Volume 2.

Washington, D.C: U.S. Department of Energy, Office of Buildings and Community Systems. Prepared by Lawrence Berkeley Laboratory, Berkeley, CA, in cooperation with American Institute of Architects Foundation and Steven Winter Associates, Inc., New York, NY, June 1989.

DOE-2 Reference Manual (Version 2.1A) Part 1. (1981). D. York and C. Cappiello, eds. Berkeley, CA: Lawrence Berkeley Laboratory.

DOE-2 Supplement (Version 2.1E). (1994). Berkeley, CA: Lawrence Berkeley Laboratory.

Duffie, J.A.; Beckman, W.A. (1980). Solar Engineering of Thermal Processes. New York, NY: John Wiley \& Sons. 
EIA (2009a). "Annual U.S. Price of Natural Gas Delivered to Consumers: Total by End-Use Sector." Washington, D.C.: Energy Information Administration. http://tonto.eia.doe.gov/dnav/ng/hist/ n3010us3m.htm. Last accessed January 2010.

EIA (2009b). "Average Retail Price of Electricity Delivered to Ultimate Consumers: Total by End-Use Sector." Washington, D.C.: Energy Information Administration. www.eia.doe.gov/cneaf/electricity/ epm/tables5 3.htm. Last accessed January 2010.

EnergyPlus Engineering Reference. (2009). Urbana, IL: University of Illinois, and Berkeley California: Lawrence Berkeley National Laboratory. http://apps1.eere.energy.gov/buildings/energyplus/pdfs/ engineeringreference.pdf. Last accessed January 2010.

EnergyPlus Input Output Reference. (2009). Urbana, IL: University of Illinois, and Berkeley, CA: Lawrence Berkeley National Laboratory. http://apps1.eere.energy.gov/buildings/energyplus/pdfs/ InputOutputReference.pdf/. Last accessed January 2010.

European Union. (2002). On the Energy Performance of Buildings. Directive 2002/91/EC of the European Parliament and of the Council, Official Journal of the European Communities, December.

Internal Revenue Service (IRS). (2008). Amplification of Notice 2006-52, Deduction for Energy Efficient Commercial Buildings. IRS Notice 2008-40. Appears in Internal Revenue Bulletin (IRB) No. 2008-14, April 7, 2008. Washington, DC: U.S. Department of Treasury, Internal Revenue Service.

Judkoff, R. (1988). "Validation of Building Energy Analysis Simulation Programs at the Solar Energy Research Institute.” Energy and Buildings, 10(3):235. Lausanne, Switzerland: Elsevier Sequoia.

Judkoff, R. (2008). “Thoughts on Validation for Retrofit: Calibration/True-Up." Presented at Affordable Comfort Conference, Pittsburgh, PA, April 2008.

Judkoff, R.; Neymark, J. (1995a). Home Energy Rating System Building Energy Simulation Test (HERS BESTEST). NREL/TP-472-7332a and TP-472-7332b. Golden, CO: National Renewable Energy Laboratory.

Judkoff, R.; Neymark, J. (1995b). International Energy Agency Building Energy Simulation Test (IEA BESTEST) and Diagnostic Method. NREL/TP-472-6231. Golden, CO: National Renewable Energy Laboratory. www.nrel.gov/docs/legosti/old/6231.pdf.

Judkoff, R.; Neymark, J. (2006). "Model Validation and Testing: The Methodological Foundation of ASHRAE Standard 140.” ASHRAE Transactions 112(2):367-376. Atlanta, GA: American Society of Heating, Refrigerating and Air-Conditioning Engineers.

Judkoff, R., Neymark, J. (2009). "What Did They Do in IEA 34/43? Or How to Diagnose and Repair Bugs in 500,000 Lines of Code." Proceedings of Building Simulation 2009. July 27-30, Glasgow, Scotland. International Building Performance Simulation Association.

Judkoff, R.; Neymark, J.; Polly, B.; Bianchi, M. (2010). Example Procedures for Developing Acceptance Range Criteria for BESTEST-EX. NREL/TP-550-47542. Golden, CO: National Renewable Energy Laboratory.

Judkoff, R.; Wortman, D.; O’Doherty, B.; Burch, J. (2008). A Methodology for Validating Building Energy Analysis Simulations. NREL/TP-550-42059. Golden, CO: National Renewable Energy Laboratory. Based on unpublished report of 1983 with same authors and title, previously referenced as SERI/TR-254-1508.

Kennedy, M.; Palmiter, L.; Wheeling, T. (1992). SUNCODE-PC Building Load Simulation Program. Available from Ecotope, Inc., 2812 E. Madison, Seattle, WA, 98112, (206) 322-3753. This software is based on SERIRES-1.0 developed at NREL. 
Kotz, S.; van Dorp, J.R. (2004). Beyond Beta, Other Continuous Families of Distributions with Bounded Support and Applications. Chapter 1, "The Triangular Distribution." Singapore: World Scientific Publishing Co.

Marion, W.; Urban, K. (1995). User's Manual for TMY2s (Typical Meteorological Years)—Derived from the 1961-1990 National Solar Radiation Data Base. NREL/TP-463-7668. Golden, CO: National Renewable Energy Laboratory.

McQuiston, F.; Spitler, J. (1992). Cooling and Heating Load Calculation Manual, Second Edition. Atlanta, GA: American Society of Heating, Refrigerating and Air-Conditioning Engineers.

Neymark, J.; Girault, P.; Guyon, G.; Judkoff, R.; LeBerre, R.; Ojalvo, J.; Reimer, P. (2005). “The ETNA BESTEST Empirical Validation Data Set.” Proceedings of Building Simulation 2005. August 15-18, 2005, Montreal, Canada. International Building Performance Simulation Association.

Neymark J.; Judkoff, R. (2002). International Energy Agency Building Energy Simulation Test and Diagnostic Method for Heating Ventilating and Air-Conditioning Equipment Models (HVAC BESTEST), Volume 1: Cases E100-E200. NREL/TP-550-30152. Golden, CO: National Renewable Energy Laboratory. www.nrel.gov/docs/fy02osti/30152.pdf.

Neymark, J.; Judkoff, R. (2004). International Energy Agency Building Energy Simulation Test and Diagnostic Method for Heating, Ventilating, and Air-Conditioning Equipment Models (HVAC BESTEST), Volume 2: Cases E300-E545. NREL/TP-550-36754. Golden, CO: National Renewable Energy Laboratory. www.nrel.gov/docs/fy05osti/36754.pdf.

Neymark, J., Judkoff, R., Beausoleil-Morrison, I., Ben-Nakhi, A., Crowley, M., Deru, M., Henninger, R., Ribberink, H., Thornton, J., Wijsman, A., Witte, M. (2008). International Energy Agency Building Energy Simulation Test and Diagnostic Method (IEA BESTEST) In-Depth Diagnostic Cases for Ground Coupled Heat Transfer Related to Slab-on-Grade Construction. NREL/TP-550-43388. Golden, CO: National Renewable Energy Laboratory. www.nrel.gov/docs/fy08osti/43388.pdf.

Neymark, J.; Judkoff, R.; Polly, B.; Bianchi, M. (2009). "BESTEST-EX: Building Energy Simulation Test for Existing Homes." Presented at Building America Quarterly Meeting, Washington, D.C., October 8, 2009.

Neymark, J.; Norton, P. (2009). "Accuracy Tests for Software That Model Retrofits to Existing Homes." Presented at RESNET Conference, New Orleans, LA, February 16, 2009.

Pigg, S. (2001). “A Rating Tale.” Home Energy. January/February 2001. Berkeley, CA: Energy Auditor and Retrofitter, Inc.

Purdy, J.; Beausoleil-Morrison, I. (2003). Building Energy Simulation Test and Diagnostic Method for Heating, Ventilation, and Air-Conditioning Equipment Models (HVAC BESTEST): Fuel-Fired Furnace Test Cases. Ottawa, Ontario, Canada: CANMET Energy Technology Centre, Natural Resources Canada. www.iea-shc.org/task22/deliverables.htm.

RESNET (2006). Mortgage Industry National Home Energy Rating Systems Standards. Oceanside, CA: Residential Energy Services Network, Inc. www.resnet.us/standards/RESNET_Mortgage_Industry_National_HERS_Standards.pdf.

RESNET (2007). Procedures for Verification of International Energy Conservation Code Performance Path Calculation Tools. RESNET Publication No. 07-003. September 2007. Oceanside, CA: Residential Energy Services Network, Inc. www1.resnet.us/standards/iecc/procedures.pdf. 
Sharp, T. (1994). The North Carolina Field Test: Field Performance of the Preliminary Version of an Advanced Weatherization Audit for the Department of Energy's Weatherization Assistance Program. ORNL/CON-362. Oak Ridge, TN: Oak Ridge National Laboratory.

Sherman, M. (1987). "Estimation of Infiltration for Leakage and Climate Indicators." Energy and Buildings 10(81). Also see Home Energy Magazine at www.homeenergy.org/archive/hem.dis.anl.gov/ eehem/94/940111.html. Last accessed January 2010.

Sherman, M. (1998). The Use of Blower-Door Data. LBNL-35173. Berkeley, CA: Lawrence Berkeley National Laboratory.

Sherman, M.H.; Grimsrud, D.T. (1980). "Infiltration-Pressurization Correlation: Simplified Physical Modeling." ASHRAE Transactions 86(2):778. Atlanta, GA: American Society of Heating, Refrigerating and Air-Conditioning Engineers.

Walker, I.S.; Wilson, D.J. (1998). "Field Validation of Equations for Stack and Wind Driven Air Infiltration Calculations." International Journal of HVAC\&R Research 4(2). Atlanta, GA: American Society of Heating, Refrigerating and Air-Conditioning Engineers.

Walton, G. (1983). Thermal Analysis Research Program Reference Manual (TARP). NBSIR 83-2655. Washington D.C.: National Bureau of Standards.

WINDOW 4.1. (1994). LBL-35298. Berkeley, CA: Lawrence Berkeley Laboratory.

WINDOW 5.2. (2005). Berkeley, CA: Lawrence Berkeley National Laboratory. http://windows.lbl.gov/ software/window/window.html. Last accessed January 2010. Also see WINDOW 5.0 User Manual (November 2001), LBNL-44789.

Yazdanian, M.; Klems, J.H. (1994). "Measurement of the Exterior Convective Film Coefficient for Windows in Low-Rise Buildings." ASHRAE Transactions 100(1):1087. 


\section{Appendix A Weather Data}

\section{A.1 Weather Data Summary}

Site and weather characteristics corresponding to Colorad.TM2 and Lasvega.TM2 weather data are summarized in Tables A-1 and A-2, respectively. Details about TMY2 (.TM2) weather data file format are included in Section A.2.

Table A-1. Site and Weather Data Summary for Colorad.TM2 Weather, Colorado Springs, Colorado (Note 1)

\begin{tabular}{||l|l||}
\hline \hline Weather Type & Cold Clear Winters \\
\hline Weather Format & Typical Meteorological Year 2 (TM2) \\
\hline Latitude & $38.8^{\circ}$ North \\
\hline Longitude & $104.7^{\circ}$ West \\
\hline Altitude & $1881 \mathrm{~m}=6171 \mathrm{ft}$ \\
\hline Time Zone & -7 \\
\hline Site & ASHRAE Terrain Class 2 (suburban/urban) \\
& $($ Notes 2,3$)$ \\
\hline Mean Annual Wind Speed & $4.36 \mathrm{~m} / \mathrm{s}=9.75 \mathrm{mph}$ \\
\hline Mean Annual Ambient Dry-Bulb Temperature & $9.0^{\circ} \mathrm{C}=48.2^{\circ} \mathrm{F}($ Note 4$)$ \\
\hline Mean Annual Daily Temperature Range & $13.2^{\circ} \mathrm{C}=23.8^{\circ} \mathrm{F}($ Note 5$)$ \\
\hline Minimum Annual Dry-Bulb Temperature & $-21.1^{\circ} \mathrm{C}=-6.0^{\circ} \mathrm{F}$ \\
\hline Maximum Annual Dry-Bulb Temperature & $34.4^{\circ} \mathrm{C}=93.9^{\circ} \mathrm{F}$ \\
\hline Maximum Annual Wind Speed & $17.5 \mathrm{~m} / \mathrm{s}=39.1 \mathrm{mph}$ \\
\hline Heating Degree Days (Base $\left.18^{\circ} \mathrm{C}=64.4^{\circ} \mathrm{F}\right)$ & $3568^{\circ} \mathrm{C} \cdot \mathrm{days}=6422^{\circ} \mathrm{F} \cdot \mathrm{days}$ \\
\hline Cooling Degree Days (Base $\left.18^{\circ} \mathrm{C}=64.4^{\circ} \mathrm{F}\right)$ & $272^{\circ} \mathrm{C} \cdot \mathrm{days}^{\prime} 490^{\circ} \mathrm{F} \cdot$ days \\
\hline Mean Annual Dew Point Temperature & $-2.3^{\circ} \mathrm{C}=27.9^{\circ} \mathrm{F}$ \\
\hline Mean Annual Relative Humidity & $50.8^{\circ} \%$ \\
\hline Global Horizontal Solar Radiation Annual Total & $547.3 \mathrm{kBtu} /\left(\mathrm{ft}^{2} \cdot \mathrm{yr}\right)($ Note 4$)$ \\
\hline Direct Normal Solar Radiation Annual Total & $670.1 \mathrm{kBtu} /\left(\mathrm{ft}^{2} \cdot \mathrm{yr}\right)($ Note 4$)$ \\
\hline Diffuse Horizontal Solar Radiation Annual Total & $172.7 \mathrm{kBtu} /\left(\mathrm{ft}^{2} \cdot \mathrm{yr}\right)($ Note 4$)$ \\
\hline \hline
\end{tabular}

Note 1: Unless otherwise noted, values are from EnergyPlus weather pre-processor statistics report.

Note 2: See ASHRAE 2005, p. 16.3. This assumption was applied for developing equivalent constant seasonal exterior surface coefficient inputs; see Appendix C, Section C.3.

Note 3: Infiltration modeling is based on Sherman-Grimsrud (1980), assuming a highly sheltered building (Shelter Class 5) in rural terrain; see Appendix D.

Note 4: Calculated value based on EnergyPlus weather pre-processor statistics report.

Note 5: Calculated value based on DOE-2.1E weather pre-processor statistics report. 
Table A-2. Site and Weather Data Summary for Lasvega.TM2 Weather, Las Vegas, Nevada (Note 1)

\begin{tabular}{||l|l||}
\hline Weather Type & Hot Dry Summers \\
\hline Weather Format & Typical Meteorological Year 2 (TM2) \\
\hline Latitude & $36.1^{\circ}$ North \\
\hline Longitude & $115.2^{\circ}$ West \\
\hline Altitude & $664 \mathrm{~m}=2178 \mathrm{ft}$ \\
\hline Time Zone & -8 \\
\hline Site & ASHRAE Terrain Class 2 (suburban $/ \mathrm{urban})$ \\
& $($ Notes 2,3$)$ \\
\hline Mean Annual Wind Speed & $4.06 \mathrm{~m} / \mathrm{s}=9.08 \mathrm{mph}$ \\
\hline Mean Annual Ambient Dry-Bulb Temperature & $19.5^{\circ} \mathrm{C}=67.1^{\circ} \mathrm{F}($ Note 4$)$ \\
\hline Mean Annual Daily Temperature Range & $13.0^{\circ} \mathrm{C}=23.4^{\circ} \mathrm{F}($ Note 5$)$ \\
\hline Minimum Annual Dry-Bulb Temperature & $-4.4^{\circ} \mathrm{C}=24.1^{\circ} \mathrm{F}$ \\
\hline Maximum Annual Dry-Bulb Temperature & $44.4^{\circ} \mathrm{C}=111.9^{\circ} \mathrm{F}$ \\
\hline Maximum Annual Wind Speed & $20.6 \mathrm{~m} / \mathrm{s}=46.1 \mathrm{mph}$ \\
\hline Heating Degree Days (Base $\left.18^{\circ} \mathrm{C}=64.4^{\circ} \mathrm{F}\right)$ & $1248^{\circ} \mathrm{C} \cdot \mathrm{days}=2246^{\circ} \mathrm{F} \cdot$ days \\
\hline Cooling Degree Days (Base $\left.18^{\circ} \mathrm{C}=64.4^{\circ} \mathrm{F}\right)$ & $1806^{\circ} \mathrm{C} \cdot \mathrm{days}=3251^{\circ} \mathrm{F} \cdot$ days \\
\hline Mean Annual Dew Point Temperature & $-1.2^{\circ} \mathrm{C}=29.8^{\circ} \mathrm{F}$ \\
\hline Mean Annual Relative Humidity & $29.4 \%$ \\
\hline Global Horizontal Solar Radiation Annual Total & $658.7 \mathrm{kBtu} /\left(\mathrm{ft}^{2} \cdot \mathrm{yr}\right)($ Note 4$)$ \\
\hline Direct Normal Solar Radiation Annual Total & $826.3 \mathrm{kBtu} /\left(\mathrm{ft}^{2} \cdot \mathrm{yr}\right)($ Note 4$)$ \\
\hline Diffuse Horizontal Solar Radiation Annual Total & $174.4 \mathrm{kBtu} /\left(\mathrm{ft}^{2} \cdot \mathrm{yr}\right)($ Note 4$)$ \\
\hline \hline
\end{tabular}

Note 1: Unless otherwise noted, values are from EnergyPlus weather pre-processor statistics report.

Note 2: See ASHRAE 2005, p. 16.3. This assumption was applied for developing equivalent constant seasonal exterior surface coefficient inputs; see Appendix C, Section C.3.

Note 3: Infiltration modeling is based on Sherman-Grimsrud (1980), assuming a highly sheltered building (Shelter Class 5) in rural terrain; see Appendix D.

Note 4: Calculated value based on EnergyPlus weather pre-processor statistics report.

Note 5: Calculated value based on DOE-2.1E weather pre-processor statistics report. 


\section{A.2 TMY2 Weather Data Format Description}

The following TMY2 format description is extracted from Section 3 of the TMY2 user manual (Marion and Urban 1995) with minor edits.

For each station, a TMY2 file contains 1 year of hourly solar radiation, illuminance, and meteorological data. The files consist of data for the typical calendar months during 1961-1990 that are concatenated to form the typical meteorological year for each station.

Each hourly record in the file contains values for solar radiation, illuminance, and meteorological elements. A two-character source and uncertainty flag is attached to each data value to indicate whether the data value was measured, modeled, or missing, and to provide an estimate of the uncertainty of the data value.

Users should be aware that the format of the TMY2 data files is different from the format used for the NSRDB and the original TMY data files.

\section{File Convention}

File naming convention uses the Weather Bureau Army Navy (WBAN) number as the file prefix, with the characters TM2 as the file extension. For example, 13876.TM2 is the TMY2 file name for Birmingham, Alabama. The TMY2 files contain computer readable ASCII characters and have a file size of 1.26 MB.

\section{File Header}

The first record of each file is the file header that describes the station. The file header contains the WBAN number, city, state, time zone, latitude, longitude, and elevation. The field positions and definitions of these header elements are given in Table A-3, along with sample FORTRAN and C formats for reading the header. A sample of a file header and data for January 1 is shown in Figure A-1.

\section{Hourly Records}

Following the file header, 8,760 hourly data records provide 1 year of solar radiation, illuminance, and meteorological data, along with their source and uncertainty flags. Table A-4 provides field positions, element definitions, and sample FORTRAN and $\mathrm{C}$ formats for reading the hourly records.

Each hourly record begins with the year (field positions 2-3) from which the typical month was chosen, followed by the month, day, and hour information in field positions 4-9. The times are in local standard time (previous TMYs based on SOLMET/ERSATZ data are in solar time). 
-6 N $43 \quad 34$ W $96 \quad 44 \quad 435$

$85010101000000000000 ? 00000 ? 00000 ? 00000 ? 00000 ? 00000 ? 00000 ? 010 \mathrm{~A} 710 \mathrm{~A} 7-150 \mathrm{~A} 7-211 \mathrm{~A} 7060 \mathrm{~A} 70975 \mathrm{~A} 7360 \mathrm{~A} 7052 \mathrm{~A} 70161 \mathrm{~A} 700945 \mathrm{~A} 70999099999004 \mathrm{E} 7050 \mathrm{~F} 8000 \mathrm{~A} 700 \mathrm{E} 7$ $85010102000000000000 ? 00000 ? 00000 ? 00000 ? 00000 ? 00000 ? 00000 ? 010 \mathrm{~A} 710 \mathrm{~A} 7-144 \mathrm{~A} 7-206 \mathrm{~A} 7060 \mathrm{~A} 70975 \mathrm{~A} 7350 \mathrm{~A} 7077 \mathrm{~A} 70161 \mathrm{~A} 700914 \mathrm{~A} 70999099999004 \mathrm{E} 7050 \mathrm{~F} 8000 \mathrm{~A} 700 \mathrm{E} 7$ $85010103000000000000 ? 00000 ? 00000 ? 00000300000 ? 00000 ? 00000 ? 010 A 710 A 7-144 A 7-200 A 7063 A 70975 A 7340 A 7062 A 70161 A 700732 A 70999099999004 \mathrm{E} 7050 F 8000 A 700 \mathrm{E} 7$ $850101040000000000003000003000003000003000003000003000003010 A 710 A 7-150 A 7-206 A 7063 A 70976 A 7330 A 7072 A 70161 A 700640 A 70999099999004 \mathrm{E} 7050 \mathrm{~F} 8000 \mathrm{~A} 700 \mathrm{E} 7$ 0
0

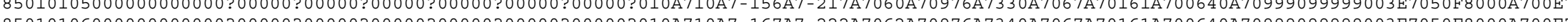

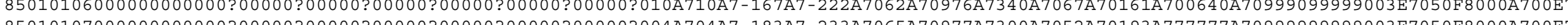

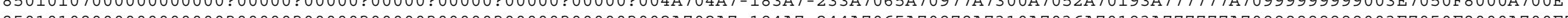

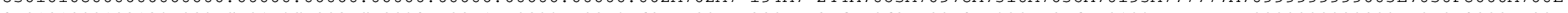

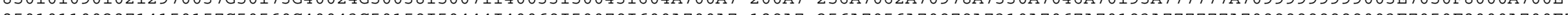
$85010110028714150157 \mathrm{G} 50560 \mathrm{G} 40043 \mathrm{G} 0159 \mathrm{I} 04441400691500791600 \mathrm{~A} 700 \mathrm{~A} 7-189 \mathrm{~A} 7-256 \mathrm{~A} 7056 \mathrm{~A} 70979 \mathrm{~A} 7310 \mathrm{~A} 7067 \mathrm{~A} 70193 \mathrm{~A} 777777 \mathrm{~A} 70999999999003 \mathrm{E} 7050 \mathrm{~F} 8000 \mathrm{~A} 700 \mathrm{E} 7$ $85010111043614150276 \mathrm{G} 40714 \mathrm{G} 40056 \mathrm{G} 502861406421400881501111500 \mathrm{~A} 700 \mathrm{~A} 7-172 \mathrm{~A} 7-250 \mathrm{~A} 7051 \mathrm{~A} 70979 \mathrm{~A} 7310 \mathrm{~A} 7062 \mathrm{~A} 70161 \mathrm{~A} 777777 \mathrm{~A} 70999999999003 \mathrm{E} 7050 \mathrm{~F} 8000 \mathrm{~A} 700 \mathrm{E} 7$ $85010112053014150357 \mathrm{G} 40782 \mathrm{G} 40064 \mathrm{G} 50374 \mathrm{I} 40735 \mathrm{I} 40098 \mathrm{I} 50131 \mathrm{I} 500 \mathrm{~A} 700 \mathrm{~A} 7-167 \mathrm{~A} 7-244 \mathrm{~A} 7051 \mathrm{~A} 70978 \mathrm{~A} 7300 \mathrm{~A} 7062 \mathrm{~A} 70161 \mathrm{~A} 777777 \mathrm{~A} 70999999999003 \mathrm{E} 7050 \mathrm{~F} 8000 \mathrm{~A} 700 \mathrm{E} 7$ $85010113056214150387 \mathrm{G} 40806 \mathrm{G} 40067 \mathrm{G} 50407$ I4 0767I40101I50139I500A700A7-156A7-244A704 7A70978A7320A7067A70193A777777A 709999999990003E7050F8000A700E $85010114053014150359 \mathrm{G} 40788 \mathrm{G} 40064 \mathrm{G} 50377$ I4 0742I40098I50131I500A700A7-144A7-239A7045A70978A7310A7062A70193A7777777A709999999999003E7050F8000A700E $85010115043614150277 G 40716 \mathrm{G} 40056 \mathrm{G} 50289140645140088150111$ I500A700A7-139A7-239A 7043A70978A7330A7052A $70193 \mathrm{~A} 777777 \mathrm{~A} 70999999999003 \mathrm{E} 7050 \mathrm{~F} 8000 \mathrm{~A} 700 \mathrm{E} 7$ $85010116028614150157 G 50564$ G40043G50162I50450I40069I50080I600A700A7-139A7-233A7045A70978A7300A7052A70161A777777A 70999999999003E7050F8000A700E $85010117010412730038 \mathrm{G} 50209 \mathrm{G} 40021 \mathrm{G} 50038 \mathrm{I} 50104$ I40030I50038I600A700A7-150A7-233A7049A70978A7290A7041A70241A777777A 70999999999003E7050F8000A700E7 $85010118000000000000 ? 00000 ? 00000 ? 00000 ? 00000 ? 00000 ? 00000 ? 000 A 700 A 7-167 A 7-233 A 7057 A 70978$ A 7000A7000A 70241A7777777A70999999999003E7050F8000A700E7 $85010119000000000000 ? 00000 ? 00000 ? 00000 ? 00000 ? 00000 ? 00000 ? 000 A 700 A 7-172 A 7-233 A 7059 A 70978$ A7000A7000A70241A7777777A70999999999003E7050F8000A700E7 $85010120000000000000 ? 00000 ? 00000 ? 00000 ? 00000 ? 00000 ? 00000 ? 000 \mathrm{~A} 700 \mathrm{~A} 7-178 \mathrm{~A} 7-233 \mathrm{~A} 7062 \mathrm{~A} 70978 \mathrm{~A} 7000 \mathrm{~A} 7000 \mathrm{~A} 70241 \mathrm{~A} 777777 \mathrm{~A} 70999999999003 \mathrm{E} 7050 \mathrm{~F} 8000 \mathrm{~A} 700 \mathrm{E} 7$ $85010121000000000000 ? 00000 ? 00000 ? 00000 ? 00000 ? 00000 ? 00000 ? 000 A 700 A 7-183 A 7-239 A 7062$ A 70978A7260A7015A70241A7777777A 70999999999003E7050F8000A700E7

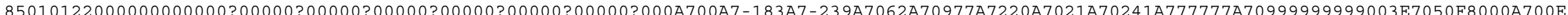
$8501012300000000000030000020000030000030000030000030000003000 A 700 A 7-178 A 7-239 A 7059 A 70977 A 7220 A 7015 A 70241 A 7777777 A 70999999999003 \mathrm{~A} 7050 F 8000 \mathrm{~A} 700 \mathrm{~B} 7$ $85010124000000000000 ? 00000 ? 00000 ? 00000 ? 00000 ? 00000 ? 00000 ? 000 A 700 A 7-178 A 7-239 A 7059 A 70977 A 7240 A 7010 A 70241$ A 7 777777A70999999999003E7050F8000A700E7

4

$6 \quad 7$

8

9

1

1

$$
1
$$

1

(for field position identification only)

Figure A-1. Sample file header and data in the TMY2 format for January 1 
Table A-3. Header Elements in the TMY2 Format (For First Record of Each File)

\begin{tabular}{|c|c|c|}
\hline Field Position & Element & Definition \\
\hline $002-006$ & WBAN Number & Station's WBAN number (see Table 2-1 of Marion and Urban [1995]) \\
\hline $008-029$ & City & City where the station is located (maximum of 22 characters) \\
\hline $031-032$ & State & State where the station is located (abbreviated to two letters) \\
\hline $034-036$ & Time Zone & $\begin{array}{l}\text { Time zone is the number of hours by which the local standard time is } \\
\text { ahead of or behind Universal Time. For example, Mountain Standard Time } \\
\text { is designated }-7 \text { because it is } 7 \text { hours behind Universal Time. }\end{array}$ \\
\hline $\begin{array}{l}038-044 \\
038 \\
040-041 \\
043-044\end{array}$ & Latitude & $\begin{array}{l}\text { Latitude of the station } \\
\mathrm{N}=\text { North of equator } \\
\text { Degrees } \\
\text { Minutes }\end{array}$ \\
\hline $\begin{array}{l}046-053 \\
046 \\
048-050 \\
052-053\end{array}$ & Longitude & $\begin{array}{l}\text { Longitude of the station } \\
W=\text { West, } E=\text { East } \\
\text { Degrees } \\
\text { Minutes }\end{array}$ \\
\hline $056-059$ & Elevation & Elevation of station in meters above sea level \\
\hline \multicolumn{3}{|c|}{ 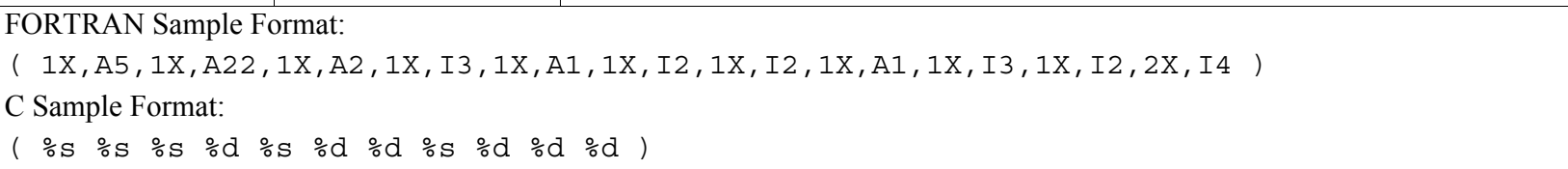 } \\
\hline
\end{tabular}

Table A-4. Data Elements in the TMY2 Format (For All Except the First Record)

\begin{tabular}{|c|c|c|c|}
\hline Field Position & Element & Values & Definition \\
\hline $\begin{array}{l}\mathbf{0 0 2}-\mathbf{0 0 9} \\
002-003 \\
004-005 \\
006-007 \\
008-009\end{array}$ & $\begin{array}{l}\text { Local Standard Time } \\
\text { Year } \\
\text { Month } \\
\text { Day } \\
\text { Hour }\end{array}$ & $\begin{array}{l}61-90 \\
1-12 \\
1-31 \\
1-24\end{array}$ & $\begin{array}{l}\text { Year, 1961-1990 } \\
\text { Month } \\
\text { Day of month } \\
\text { Hour of day in local standard time }\end{array}$ \\
\hline $010-013$ & $\begin{array}{l}\text { Extraterrestrial Horizontal } \\
\text { Radiation }\end{array}$ & $0-1415$ & $\begin{array}{l}\text { Amount of solar radiation in } \mathrm{Wh} / \mathrm{m}^{2} \text { received } \\
\text { on a horizontal surface at the top of the } \\
\text { atmosphere during the } 60 \text { minutes preceding } \\
\text { the hour indicated }\end{array}$ \\
\hline $014-017$ & $\begin{array}{l}\text { Extraterrestrial Direct Normal } \\
\text { Radiation }\end{array}$ & $0-1415$ & $\begin{array}{l}\text { Amount of solar radiation in } \mathrm{Wh} / \mathrm{m}^{2} \text { received } \\
\text { on a surface normal to the sun at the top of the } \\
\text { atmosphere during the } 60 \text { minutes preceding } \\
\text { the hour indicated }\end{array}$ \\
\hline $\begin{array}{l}018-023 \\
018-021 \\
022 \\
023\end{array}$ & $\begin{array}{l}\text { Global Horizontal Radiation } \\
\text { Data Value } \\
\text { Flag for Data Source } \\
\text { Flag for Data Uncertainty }\end{array}$ & $\begin{array}{c}0-1200 \\
A-H, ? \\
0-9\end{array}$ & $\begin{array}{l}\text { Total amount of direct and diffuse solar } \\
\text { radiation in } \mathrm{Wh} / \mathrm{m}^{2} \text { received on a horizontal } \\
\text { surface during the } 60 \text { minutes preceding the } \\
\text { hour indicated }\end{array}$ \\
\hline $\begin{array}{l}024-029 \\
024-027 \\
028 \\
029\end{array}$ & $\begin{array}{l}\text { Direct Normal Radiation } \\
\text { Data Value } \\
\text { Flag for Data Source } \\
\text { Flag for Data Uncertainty }\end{array}$ & $\begin{array}{c}0-1100 \\
A-H, ? \\
0-9\end{array}$ & $\begin{array}{l}\text { Amount of solar radiation in } \mathrm{Wh} / \mathrm{m}^{2} \text { received } \\
\text { within a } 5.7^{\circ} \text { field of view centered on the sun, } \\
\text { during the } 60 \text { minutes preceding the hour } \\
\text { indicated }\end{array}$ \\
\hline
\end{tabular}


Table A-4. Data Elements in the TMY2 Format (Continued)

\begin{tabular}{|c|c|c|c|}
\hline Field Position & Element & Values & Definition \\
\hline $\begin{array}{l}030-035 \\
030-033 \\
034 \\
035\end{array}$ & $\begin{array}{l}\text { Diffuse Horizontal Radiation } \\
\text { Data Value } \\
\text { Flag for Data Source } \\
\text { Flag for Data Uncertainty }\end{array}$ & $\begin{array}{c}0-700 \\
\text { A }-\mathrm{H}, ? \\
0-9\end{array}$ & $\begin{array}{l}\text { Amount of solar radiation in } \mathrm{Wh} / \mathrm{m}^{2} \\
\text { received from the sky (excluding the } \\
\text { solar disk) on a horizontal surface } \\
\text { during the } 60 \text { minutes preceding the } \\
\text { hour indicated }\end{array}$ \\
\hline $\begin{array}{l}036-041 \\
036-039 \\
040 \\
041\end{array}$ & $\begin{array}{l}\text { Global Horiz. Illuminance } \\
\text { Data Value } \\
\text { Flag for Data Source } \\
\text { Flag for Data Uncertainty }\end{array}$ & $\begin{array}{l}0-1,300 \\
\text { I, ? } \\
0-9\end{array}$ & $\begin{array}{l}\text { Average total amount of direct and } \\
\text { diffuse illuminance in hundreds of lux } \\
\text { received on a horizontal surface } \\
\text { during the } 60 \text { minutes preceding the } \\
\text { hour indicated. } \\
0 \text { to } 1,300=0 \text { to } 130,000 \text { lux }\end{array}$ \\
\hline $\begin{array}{l}042-047 \\
042-045 \\
046 \\
047\end{array}$ & $\begin{array}{l}\text { Direct Normal Illuminance } \\
\text { Data Value } \\
\text { Flag for Data Source } \\
\text { Flag for Data Uncertainty }\end{array}$ & $\begin{array}{c}0-1,100 \\
\text { I, ? } \\
0-9\end{array}$ & $\begin{array}{l}\text { Average amount of direct normal } \\
\text { illuminance in hundreds of lux } \\
\text { received within a } 5.7 \text { degree field of } \\
\text { view centered on the sun during the } \\
60 \text { minutes preceding the hour } \\
\text { indicated. } \\
0 \text { to } 1,100=0 \text { to } 110,000 \text { lux }\end{array}$ \\
\hline $\begin{array}{l}048-053 \\
048-051 \\
052 \\
053\end{array}$ & $\begin{array}{l}\text { Diffuse Horiz. Illuminance } \\
\text { Data Value } \\
\text { Flag for Data Source } \\
\text { Flag for Data Uncertainty }\end{array}$ & $\begin{array}{l}0-800 \\
\quad \text { I, ? } \\
0-9\end{array}$ & $\begin{array}{l}\text { Average amount of illuminance in } \\
\text { hundreds of lux received from the sky } \\
\text { (excluding the solar disk) on a } \\
\text { horizontal surface during the } 60 \\
\text { minutes preceding the hour indicated. } \\
0 \text { to } 800=0 \text { to } 80,000 \text { lux }\end{array}$ \\
\hline $\begin{array}{l}\mathbf{0 5 4 - 0 5 9} \\
054-057 \\
058 \\
059\end{array}$ & $\begin{array}{l}\text { Zenith Luminance } \\
\text { Data Value } \\
\text { Flag for Data Source } \\
\text { Flag for Data Uncertainty }\end{array}$ & $\begin{array}{c}0-7,000 \\
\text { I,? } \\
0-9\end{array}$ & $\begin{array}{l}\text { Average amount of luminance at the } \\
\text { sky's zenith in tens of } \mathrm{Cd} / \mathrm{m}^{2} \text { during } \\
\text { the } 60 \text { minutes preceding the hour } \\
\text { indicated. } \\
0 \text { to } 7,000=0 \text { to } 70,000 \mathrm{Cd} / \mathrm{m}^{2}\end{array}$ \\
\hline $\begin{array}{l}060-063 \\
060-061 \\
062 \\
063\end{array}$ & $\begin{array}{l}\text { Total Sky Cover } \\
\text { Data Value } \\
\text { Flag for Data Source } \\
\text { Flag for Data Uncertainty }\end{array}$ & $\begin{array}{c}0-10 \\
A-F, ? \\
0-9\end{array}$ & $\begin{array}{l}\text { Amount of sky dome in tenths covered } \\
\text { by clouds or obscuring phenomena at } \\
\text { the hour indicated }\end{array}$ \\
\hline $\begin{array}{l}064-067 \\
064-065 \\
066 \\
067\end{array}$ & $\begin{array}{l}\text { Opaque Sky Cover } \\
\text { Data Value } \\
\text { Flag for Data Source } \\
\text { Flag for Data Uncertainty }\end{array}$ & $\begin{array}{l}0-10 \\
A-F \\
0-9\end{array}$ & $\begin{array}{l}\text { Amount of sky dome in tenths covered } \\
\text { by clouds or obscuring phenomena } \\
\text { that prevent observing the sky or } \\
\text { higher cloud layers at the hour } \\
\text { indicated }\end{array}$ \\
\hline $\begin{array}{l}068-073 \\
068-071 \\
072 \\
073\end{array}$ & $\begin{array}{l}\text { Dry-Bulb Temperature } \\
\text { Data Value } \\
\text { Flag for Data Source } \\
\text { Flag for Data Uncertainty }\end{array}$ & $\begin{array}{l}-500 \text { to } 500 \\
\text { A - F } \\
0-9\end{array}$ & $\begin{array}{l}\text { Dry-bulb temperature in tenths of }{ }^{\circ} \mathrm{C} \\
\text { at the hour indicated. } \\
-500 \text { to } 500=-50.0 \text { to } 50.0^{\circ} \mathrm{C}\end{array}$ \\
\hline $\begin{array}{l}\mathbf{0 7 4 - 0 7 9} \\
074-077 \\
078 \\
079\end{array}$ & $\begin{array}{l}\text { Dew Point Temperature } \\
\text { Data Value } \\
\text { Flag for Data Source } \\
\text { Flag for Data Uncertainty }\end{array}$ & $\begin{array}{l}-600 \text { to } 300 \\
\text { A - F } \\
0-9\end{array}$ & $\begin{array}{l}\text { Dew point temperature in tenths of }{ }^{\circ} \mathrm{C} \\
\text { at the hour indicated. } \\
-600 \text { to } 300=-60.0 \text { to } 30.0^{\circ} \mathrm{C}\end{array}$ \\
\hline $\begin{array}{l}080-084 \\
080-082 \\
083 \\
084\end{array}$ & $\begin{array}{l}\text { Relative Humidity } \\
\text { Data Value } \\
\text { Flag for Data Source } \\
\text { Flag for Data Uncertainty }\end{array}$ & $\begin{array}{l}0-100 \\
\text { A - F } \\
0-9\end{array}$ & $\begin{array}{l}\text { Relative humidity in percent at the } \\
\text { hour indicated }\end{array}$ \\
\hline
\end{tabular}


Table A-4. Data Elements in the TMY2 Format (Continued)

\begin{tabular}{|c|c|c|c|}
\hline Field Position & Element & Values & Definition \\
\hline $\begin{array}{l}085-090 \\
085-088 \\
089 \\
090\end{array}$ & $\begin{array}{l}\text { Atmospheric Pressure } \\
\text { Data Value } \\
\text { Flag for Data Source } \\
\text { Flag for Data Uncertainty }\end{array}$ & $\begin{array}{c}700-1100 \\
A-F \\
0-9\end{array}$ & $\begin{array}{l}\text { Atmospheric pressure at station in millibars at } \\
\text { the hour indicated }\end{array}$ \\
\hline $\begin{array}{l}091-095 \\
091-093 \\
094 \\
095\end{array}$ & $\begin{array}{l}\text { Wind Direction } \\
\text { Data Value } \\
\text { Flag for Data Source } \\
\text { Flag for Data Uncertainty }\end{array}$ & $\begin{array}{l}0-360 \\
A-F \\
0-9\end{array}$ & $\begin{array}{l}\text { Wind direction in degrees at the hour } \\
\text { indicated. }(N=0 \text { or } 360, E=90, S=180, W= \\
270) . \text { For calm winds, wind direction equals } \\
\text { zero. }\end{array}$ \\
\hline $\begin{array}{l}096-100 \\
096-98 \\
99 \\
100\end{array}$ & $\begin{array}{l}\text { Wind Speed } \\
\text { Data Value } \\
\text { Flag for Data Source } \\
\text { Flag for Data Uncertainty }\end{array}$ & $\begin{array}{l}0-400 \\
A-F \\
0-9\end{array}$ & $\begin{array}{l}\text { Wind speed in tenths of meters per second at } \\
\text { the hour indicated. } \\
0 \text { to } 400=0 \text { to } 40.0 \mathrm{~m} / \mathrm{s}\end{array}$ \\
\hline $\begin{array}{l}101-106 \\
101-104 \\
105 \\
106\end{array}$ & $\begin{array}{l}\text { Visibility } \\
\text { Data Value } \\
\text { Flag for Data Source } \\
\text { Flag for Data Uncertainty }\end{array}$ & $\begin{array}{c}0-1609 \\
A-F, ? \\
0-9\end{array}$ & $\begin{array}{l}\text { Horizontal visibility in tenths of kilometers at } \\
\text { the hour indicated. } \\
7777=\text { unlimited visibility } \\
0 \text { to } 1609=0.0 \text { to } 160.9 \mathrm{~km} \\
9999=\text { missing data }\end{array}$ \\
\hline $\begin{array}{l}107-113 \\
107-111 \\
112 \\
113\end{array}$ & $\begin{array}{l}\text { Ceiling Height } \\
\text { Data Value } \\
\text { Flag for Data Source } \\
\text { Flag for Data Uncertainty }\end{array}$ & $\begin{array}{l}0-30450 \\
A-F, ? \\
0-9\end{array}$ & $\begin{array}{l}\text { Ceiling height in meters at the hour indicated. } \\
77777 \text { = unlimited ceiling height } \\
88888 \text { = cirroform } \\
99999=\text { missing data }\end{array}$ \\
\hline $114-123$ & Present Weather & $\begin{array}{c}\text { See } \\
\text { Appendix B of } \\
\text { Marion and } \\
\text { Urban (1995) }\end{array}$ & $\begin{array}{l}\text { Present weather conditions denoted by a 10- } \\
\text { digit number. See Appendix B of Marion and } \\
\text { Urban (1995) for key to present weather } \\
\text { elements. }\end{array}$ \\
\hline $\begin{array}{l}124-128 \\
124-126 \\
127 \\
128\end{array}$ & $\begin{array}{l}\text { Precipitable Water } \\
\text { Data Value } \\
\text { Flag for Data Source } \\
\text { Flag for Data Uncertainty }\end{array}$ & $\begin{array}{l}0-100 \\
A-F \\
0-9\end{array}$ & $\begin{array}{l}\text { Precipitable water in millimeters at the hour } \\
\text { indicated }\end{array}$ \\
\hline $\begin{array}{l}129-133 \\
129-131 \\
132 \\
133\end{array}$ & $\begin{array}{l}\text { Aerosol Optical Depth } \\
\text { Data Value } \\
\text { Flag for Data Source } \\
\text { Flag for Data Uncertainty }\end{array}$ & $\begin{array}{l}0-240 \\
A-F \\
0-9\end{array}$ & $\begin{array}{l}\text { Broadband aerosol optical depth (broad-band } \\
\text { turbidity) in thousandths on the day indicated. } \\
0 \text { to } 240=0.0 \text { to } 0.240\end{array}$ \\
\hline $\begin{array}{l}134-138 \\
134-136 \\
137 \\
138\end{array}$ & $\begin{array}{l}\text { Snow Depth } \\
\text { Data Value } \\
\text { Flag for Data Source } \\
\text { Flag for Data Uncertainty }\end{array}$ & $\begin{array}{c}0-150 \\
A-F, ? \\
0-9\end{array}$ & $\begin{array}{l}\text { Snow depth in centimeters on the day } \\
\text { indicated. } \\
999=\text { missing data }\end{array}$ \\
\hline $\begin{array}{l}139-142 \\
139-140 \\
141 \\
142\end{array}$ & $\begin{array}{l}\text { Days Since Last Snowfall } \\
\text { Data Value } \\
\text { Flag for Data Source } \\
\text { Flag for Data Uncertainty }\end{array}$ & $\begin{array}{c}0-88 \\
A-F, ? \\
0-9\end{array}$ & $\begin{array}{l}\text { Number of days since last snowfall } \\
88=88 \text { or greater days } \\
99=\text { missing data }\end{array}$ \\
\hline \multicolumn{4}{|c|}{ 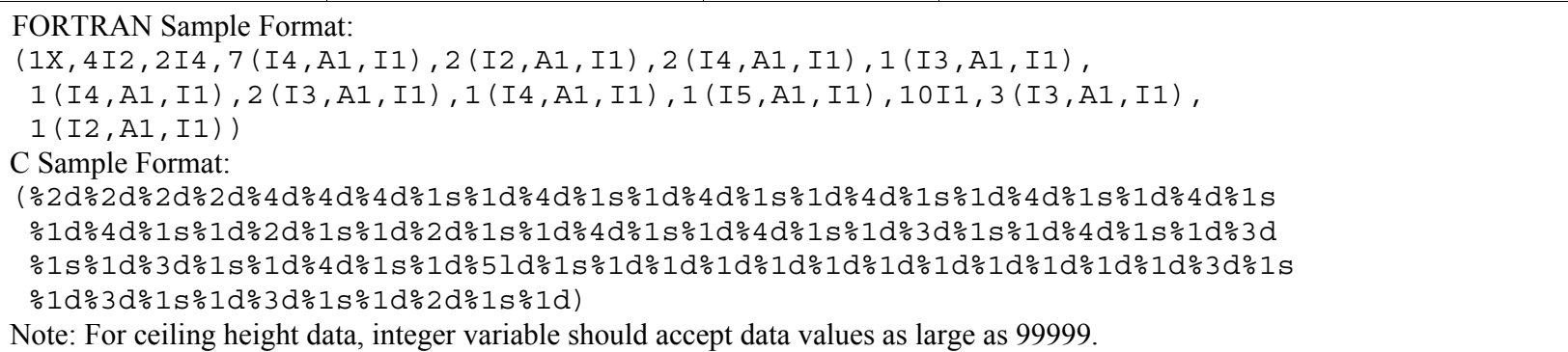 } \\
\hline
\end{tabular}


For solar radiation and illuminance elements, the data values represent the energy received during the 60 minutes preceding the hour indicated. For meteorological elements (with a few exceptions), observations or measurements were made at the hour indicated. A few of the meteorological elements had observations, measurements, or estimates made at daily, instead of hourly, intervals. Consequently, the data values for broadband aerosol optical depth, snow depth, and days since last snowfall represent the values available for the day indicated.

\section{Missing Data}

Data for some stations, times, and elements are missing. The causes for missing data include such things as equipment problems, some stations not operating at night, and a National Oceanic and Atmospheric Administration (NOAA) cost-saving effort from 1965 to 1981 that digitized data for only every third hour.

Although both the National Solar Radiation Database (NSRDB) and the TMY2 data sets used methods to fill data where possible, some elements, because of their discontinuous nature, did not lend themselves to interpolation or other data-filling methods. Consequently, data in the TMY2 data files may be missing for horizontal visibility, ceiling height, and present weather for up to 2 consecutive hours for Class A stations and for up to 47 hours for Class B stations. For Colorado Springs, Colorado, snow depth and days since last snowfall may also be missing. No data are missing for more than 47 hours, except for snow depth and days since last snowfall for Colorado Springs, Colorado. As indicated in Table A-4, missing data values are represented by 9's and the appropriate source and uncertainty flags.

\section{Source and Uncertainty Flags}

With the exception of extraterrestrial horizontal and extraterrestrial direct radiation, the two field positions immediately following the data value provide source and uncertainty flags both to indicate whether the data were measured, modeled, or missing, and to provide an estimate of the uncertainty of the data. Source and uncertainty flags for extraterrestrial horizontal and extraterrestrial direct radiation are not provided because these elements were calculated using equations considered to give exact values.

For the most part, the source and uncertainty flags in the TMY2 data files are the same as the ones in NSRDB, from which the TMY2 files were derived. However, differences do exist for data that were missing in the NSRDB, but then filled while developing the TMY2 data sets. Uncertainty values apply to the data with respect to when the data were measured, and not as to how "typical" a particular hour is for a future month and day. More information on data filling and the assignment of source and uncertainty flags is found in Appendix A of Marion and Urban (1995).

Tables A-5 through A-8 define the source and uncertainty flags for the solar radiation, illuminance, and meteorological elements. 
Table A-5. Solar Radiation and Illuminance Source Flags

\begin{tabular}{|c|l|}
\hline Flag & \multicolumn{1}{c|}{ Definition } \\
\hline A & $\begin{array}{l}\text { Post-1976 measured solar radiation data as received from NCDC } \\
\text { or other sources }\end{array}$ \\
\hline B & $\begin{array}{l}\text { Same as "A" except the global horizontal data underwent a } \\
\text { calibration correction }\end{array}$ \\
\hline C & $\begin{array}{l}\text { Pre-1976 measured global horizontal data (direct and diffuse were } \\
\text { not measured before 1976), adjusted from solar to local time, } \\
\text { usually with a calibration correction }\end{array}$ \\
\hline D & $\begin{array}{l}\text { Data derived from the other two elements of solar radiation using } \\
\text { the relationship, global = diffuse + direct } \times \text { cosine (zenith) }\end{array}$ \\
\hline E & $\begin{array}{l}\text { Modeled solar radiation data using inputs of observed sky cover } \\
\text { (cloud amount) and aerosol optical depths derived from direct } \\
\text { normal data collected at the same location }\end{array}$ \\
\hline F & $\begin{array}{l}\text { Modeled solar radiation data using interpolated sky cover and } \\
\text { aerosol optical depths derived from direct normal data collected at } \\
\text { the same location }\end{array}$ \\
\hline G & $\begin{array}{l}\text { Modeled solar radiation data using observed sky cover and aerosol } \\
\text { optical depths estimated from geographical relationships }\end{array}$ \\
\hline H & $\begin{array}{l}\text { Modeled solar radiation data using interpolated sky cover and } \\
\text { estimated aerosol optical depths }\end{array}$ \\
\hline I & $\begin{array}{l}\text { Modeled illuminance or luminance data derived from measured or } \\
\text { modeled solar radiation data }\end{array}$ \\
\hline$?$ & $\begin{array}{l}\text { Source does not fit any of the above categories. Used for nighttime } \\
\text { values, calculated extraterrestrial values, and missing data }\end{array}$ \\
\hline
\end{tabular}

Table A-6. Solar Radiation and Illuminance Uncertainty Flags

\begin{tabular}{|c|c|}
\hline Flag & Uncertainty Range (\%) \\
\hline 1 & Not used \\
2 & $2-4$ \\
3 & $4-6$ \\
4 & $6-9$ \\
5 & $9-13$ \\
6 & $13-18$ \\
7 & $18-25$ \\
8 & $25-35$ \\
9 & $35-50$ \\
0 & Not applicable \\
\hline
\end{tabular}


Table A-7. Meteorological Source Flags

\begin{tabular}{|c|l|}
\hline Flag & \multicolumn{1}{|c|}{ Definition } \\
\hline A & Data as received from NCDC, converted to SI units \\
\hline B & Linearly interpolated \\
\hline C & $\begin{array}{l}\text { Non-linearly interpolated to fill data gaps from 6 to 47 hours in } \\
\text { length }\end{array}$ \\
\hline D & Not used \\
\hline E & $\begin{array}{l}\text { Modeled or estimated, except: precipitable water, calculated } \\
\text { from radiosonde data; dew point temperature calculated from } \\
\text { dry-bulb temperature and relative humidity; and relative } \\
\text { humidity calculated from dry-bulb temperature and dew point } \\
\text { temperature }\end{array}$ \\
\hline F & $\begin{array}{l}\text { Precipitable water, calculated from surface vapor pressure; } \\
\text { aerosol optical depth, estimated from geographic correlation }\end{array}$ \\
\hline$?$ & $\begin{array}{l}\text { Source does not fit any of the above. Used mostly for missing } \\
\text { data }\end{array}$ \\
\hline
\end{tabular}

Table A-8. Meteorological Uncertainty Flags

\begin{tabular}{|c|l|}
\hline Flag & \multicolumn{1}{|c|}{ Definition } \\
\hline $1-6$ & Not used \\
\hline 7 & $\begin{array}{l}\text { Uncertainty consistent with National Weather Service } \\
\text { practices and the instrument or observation used to obtain the } \\
\text { data }\end{array}$ \\
\hline 8 & $\begin{array}{l}\text { Greater uncertainty than 7 because values were interpolated } \\
\text { or estimated }\end{array}$ \\
\hline 9 & Greater uncertainty than 8 or unknown \\
\hline 0 & Not definable \\
\hline
\end{tabular}




\section{Appendix B Selection of Internal Loads}

The internal loads described in the test specification result from equipment, lights, people, etc. Values and schedules are based on information available in the literature (DOE 1989, BA 2009). Specifically, the daily total internal loads were calculated from information in Appendix D of the report, Affordable Housing Through Energy Conservation (DOE 1989). Details of these calculations are described below. An adjustment was made to the approximate input range for the sensible internal gains from electricity based on consultation with the BESTEST-EX Working Group (2009). Normalized hourly profiles were adapted from the Building America Analysis Spreadsheet (BA 2009) for internal loads caused by occupants, electricity, and gas. A discussion of these profiles is included in this appendix.

Although latent loads are developed and discussed in this appendix, they are not applied in the test specification (see Section 1.2.1.8.2). Future versions of BESTEST-EX may use the latent loads and profiles developed in this appendix.

\section{B.1 Calculation of Daily Internal Gains}

Table D.a in Appendix D of DOE (1989) was modified to calculate the daily internal gains for BESTESTEX. These results are referred to as "B-EX" internal loads. The following items are considered, and their contributions to the daily internal loads are shown in Table B-1:

- One refrigerator

- One electric range

- One gas domestic hot water system

- One electric dryer

- One television

- Miscellaneous (assumed to be electric)

- Lighting

- People.

Table B-1. Breakdown of B-EX Daily Internal Loads

\begin{tabular}{|l|c|c|}
\hline \multicolumn{1}{|c|}{ Source } & $\begin{array}{c}\text { Daily Sensible } \\
\text { Load (Btu/day) }\end{array}$ & $\begin{array}{c}\text { Daily Latent Load } \\
\text { (Btu/day) }\end{array}$ \\
\hline New Refrigerator & 10,517 & 0 \\
Range (electric) & 7,479 & 3,739 \\
DHW (gas) & 14,928 & 1,301 \\
Dryer (electric) & 841 & 0 \\
Television & 1,870 & 0 \\
Miscellaneous & 2,804 & 0 \\
Lighting & 12,957 & 0 \\
People & 8,694 & 6,871 \\
& & $\mathbf{1 1 , 9 1 1}$ \\
Total & $\mathbf{6 0 , 0 9 0}$ & \\
\hline
\end{tabular}

The total B-EX internal sensible load of $60 \mathrm{kBtu} /$ day is greater than the DOE (1989) value of $56 \mathrm{kBtu} / \mathrm{day}$ calculated for the average family home. This difference appears because specific systems were chosen in the B-EX analysis. Thus, the number of refrigerators, ranges, etc. per household is no longer fractional, as it was in the DOE calculation for the average family home. Decisions were made about the location of the equipment for BESTEST-EX: all equipment except for 10\% of the lighting is located inside the conditioned zone. Key differences for each item affecting the sensible internal gains are listed below. 
Freezer and Old Refrigerator

Unlike the DOE (1989) report, no old refrigerator or freezer is modeled. This represents an approximate $2.3 \mathrm{kBtu} /$ day decrease when B-EX is compared to DOE (1989).

Range

Modeling one electric range for B-EX leads to a negligible difference when compared to DOE (1989).

Water Heater

DOE (1989) assumes sensible gains come from standby losses and the heating of incoming cold water. 22.5 therms/yr/system is the assumed sensible load caused by standby losses from water heaters (50\% of the gas units are assumed to be outside the conditioned space). The other portion of the sensible gains comes from the energy used to heat the incoming cold water. Most of this energy leaves the zone when water goes down the drain, but 9.5 therms/yr/system is assumed to be sensible gains in the conditioned space. This leads to 32 therms/yr/system.

For one gas water heating system located in the conditioned space, the sensible gains caused by standby losses would be 45 therms/yr (twice that of DOE [1989]), for a total of 54.5 therms/yr. This corresponds to an approximate $6.1 \mathrm{kBtu} /$ day increase when B-EX is compared to DOE (1989).

Dryer

One electric dryer is assumed in the conditioned space, which represents a $0.3 \mathrm{kBtu}$ increase between BEX and DOE (1989) report.

\section{Television, Miscellaneous, Lighting, and People}

For these categories the values used in B-EX are identical to those in the DOE (1989) report.

\section{Summary}

The differences in sensible internal loads described above account for the $4 \mathrm{kBtu}$ net increase in sensible internal gains when B-EX is compared to DOE (1989).

\section{B.2 Building America Internal Gains}

DOE (1989) was used because it offers a straightforward method to calculate the internal gains without requiring many more additional assumptions about the building. However, because internal gains may change with the introduction of new and improved technology, it was important to compare these results to a newer reference. The Building America Analysis Spreadsheet (BA 2009) was used to perform a rough check on the internal gains caused by occupants, electricity, and gas. Engineering judgment was used for the numerous additional inputs required by the spreadsheet. The effect of sensible tank standby losses was also added to the spreadsheet using the relationship

$$
Q_{\text {tank }} \cong U A \Delta T(\mathrm{Btu} / \mathrm{h})
$$

where a $\Delta T$ of $52^{\circ} \mathrm{F}$ was assumed based on a tank water temperature of $120^{\circ} \mathrm{F}$ and a zone temperature of $68^{\circ} \mathrm{F}$. Tables B-2 and B-3 show the results of the DOE calculation versus the results of the Building America (BA) calculation for sensible and latent internal gains, respectively. In general the BA analysis predicts higher internal sensible and latent loads than those calculated by DOE (1989), but the percent distribution between occupants, electricity, and gas show good agreement. 
Table B-2. Comparison of Proposed Internal Sensible Loads With Building America Prototype House Results

\begin{tabular}{|lcccc|}
\hline & Occupants & Electricity & Gas & Total \\
\hline BESTEST-EX & & & & \\
(Btu/day) & 8,694 & 36,468 & 14,928 & 60,090 \\
\% of Total & 14.5 & 60.7 & 24.8 & \\
\hline $\begin{array}{l}\text { BA Analysis } \\
\text { (Btu/day) }\end{array}$ & 10,805 & 45,661 & 18,308 & 74,775 \\
\% of Total & 14.4 & 61.1 & 24.5 & \\
\hline
\end{tabular}

Table B-3. Comparison of Proposed Internal Latent Loads With Building America Prototype House Results

\begin{tabular}{|lcccc|}
\hline & $\begin{array}{c}\text { Occupants } \\
\text { Latent }\end{array}$ & $\begin{array}{c}\text { Electricity } \\
\text { Latent }\end{array}$ & $\begin{array}{c}\text { Gas } \\
\text { Latent }\end{array}$ & Total \\
\hline $\begin{array}{l}\text { BESTEST-EX } \\
\text { (Btu/day) } \\
\text { \% of Total }\end{array}$ & 6,871 & 3,739 & 1,301 & 11,911 \\
\hline $\begin{array}{l}\text { BA Analysis } \\
\text { (Btu/day) }\end{array}$ & 57.7 & 31.4 & 10.9 & \\
\% of Total & 8,075 & 3,783 & 1,689 & 13,547 \\
\hline
\end{tabular}

\section{B.3 Normalized Hourly Profiles}

The normalized hourly profiles for internal loads caused by occupants, electricity, and gas in the test specification are adapted from the BA Benchmark Analysis. For BESTEST-EX, the only non-HVAC equipment using gas is the DHW system. The hourly schedule for the sensible loads caused by gas is a load-weighted combination of the BA schedule "Combined DHW," which accounts for hot water use, and an assumed uniform schedule for standby losses. Because latent internal gains from gas (DHW) are closely related to gas usage, the BA schedule "Combined DHW" is used for the gas latent internal gains. The hourly schedule for the sensible loads from electricity is a load-weighted combination of the BA schedules for "Lights" and "Equipment Sensible Load (no lights, water heater, or occupancy)." The hourly schedule for latent loads from electricity is the BA schedule "Equipment Latent Load (no lights, water heater, or occupancy)." The BA schedule "Occ" is used for sensible and latent loads caused by occupants. Finally, a few normalized hourly load fraction values were varied slightly in Table 1-9b to obtain a daily sum of 1.000 for each load type. These schedules are plotted in Figure B-1. Occupant loads are highest in the late evening/early morning hours; electric and gas loads peak near breakfast and dinner. 


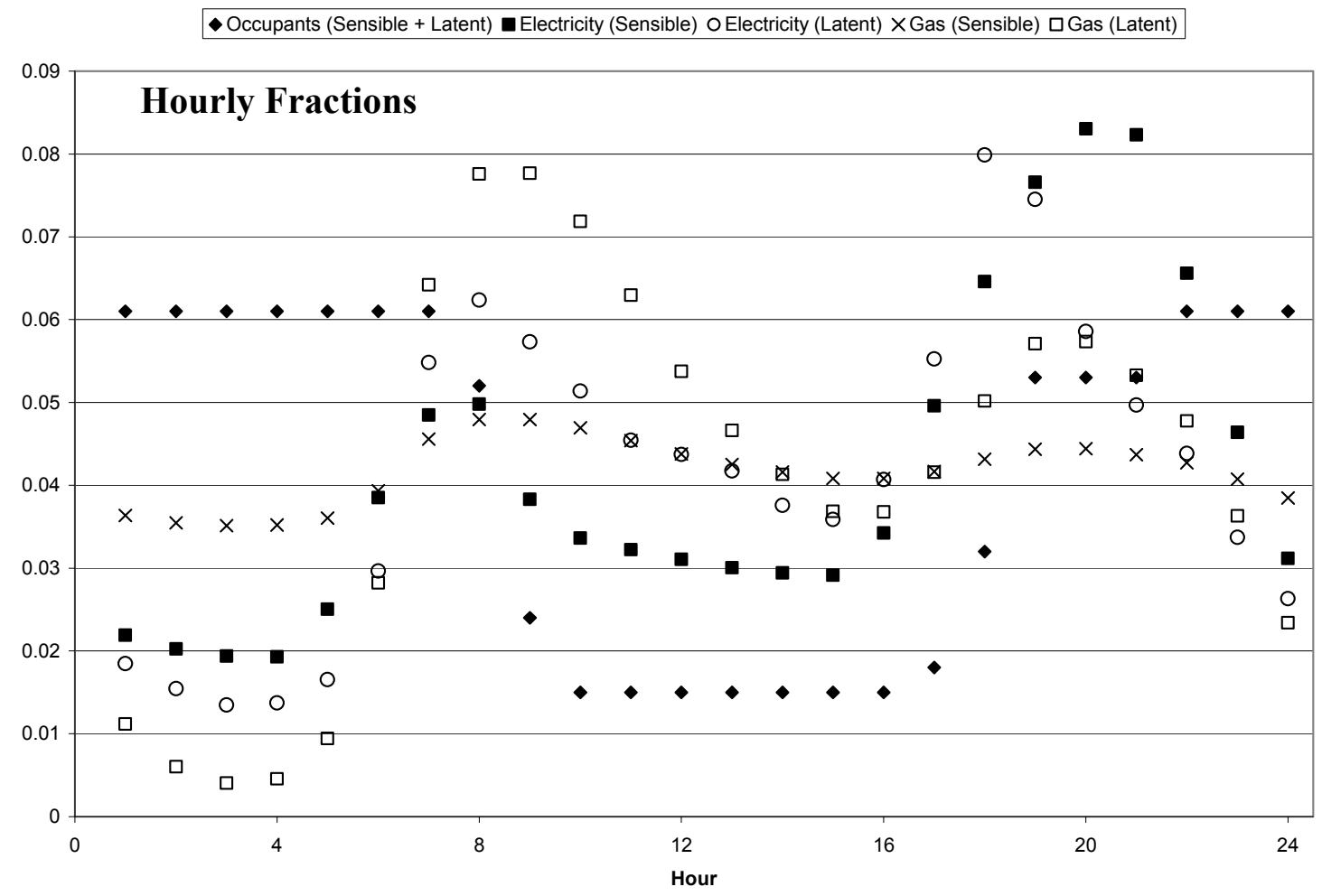

Figure B-1. Normalized hourly profiles for internal loads due to occupants, gas, and electricity

\section{B.4 Conversion Metered Gas and Electricity Use to Internal Gains}

Only a fraction of the metered gas and electricity use is converted to internal sensible gains. The percentage of energy converted depends largely on the performance, use, and location of the equipment (inside or outside the conditioned zone). For example, a house with a gas water heater in the garage could have a significantly lower percentage of non-HVAC gas energy converted to sensible internal gains than an identical house with the same water heater in the conditioned zone. Analysis of B-EX internal loads indicates that $74 \%$ of the metered non-HVAC electric energy and $20 \%$ of the metered non-HVAC gas energy is converted to sensible internal gains. A rough comparison using the BA Analysis Spreadsheet yields the same electric conversion factor of $74 \%$. The BA conversion factor for gas is not readily available, because the BA Analysis Spreadsheet does not calculate the total DHW energy consumption. 


\section{Appendix C Combined Surface Heat Transfer Coefficients}

This appendix documents the development of equivalent constant combined interior and exterior surface coefficients for programs that do not automatically calculate surface heat transfer. Using combined coefficients is an idealized simplification of the convective and radiative heat transfer at the surfaces. Assumptions about convective and radiative components of the combined coefficients are provided. If the program being tested requires disaggregated (separate convective and radiative) surface coefficients, values provided in the tables included in this appendix may be used. Other disaggregated coefficients may also be used if there is a physically logical basis for selecting them.

This appendix also documents modeling of surface heat transfer with EnergyPlus that supports development of the equivalent constant combined coefficients. For programs that use detailed inputs for automated calculation algorithms, Section C.3 includes relevant terrain assumptions that may be used for correcting wind speed given in the weather data. Appendix C and Appendix D (related to infiltration modeling) were developed based on initial simulations with EnergyPlus; EnergyPlus has different assumptions and definitions for parameters used for correcting wind speed for infiltration and exterior surface coefficient modeling. This resulted in different wind speed adjustments for modeling infiltration and exterior coefficients in the EnergyPlus reference model.

\section{C.1 Interior Surface Coefficients}

Table C1-1 gives combined convective and radiative interior surface heat transfer coefficients $\left(\mathrm{h}_{\mathrm{s}}\right)$, along with disaggregated convective $\left(h_{c}\right)$ and radiative $\left(h_{i}\right)$ coefficients. These coefficients are calculated by adding the constant equivalent convective portions (derived from EnergyPlus simulations, see Section C.1.1) with the calculated radiative portions (based on HERS BESTEST Appendix D, see Section C.1.2 below). The BESTEST-EX coefficients are displayed under the heading "B-EX." For historical reference, the original HERS BESTEST coefficients are also shown in italics under the heading "HERS." Table C11 indicates a substantial difference between interior surface coefficients of HERS BESTEST — which are based on ASHRAE coefficients used for developing design loads - versus the interior surface coefficients calculated for BESTEST-EX. Additional research is needed to understand these differences.

A simplifying assumption for direct addition of convective and radiative surface coefficients to develop combined interior surface coefficients for BESTEST-EX is that for the radiative portion for a given surface, the mean radiant surface temperature of all other surfaces (for internal surfaces and the insidefacing portion of exterior surfaces) equals the zone air temperature. As the actual mean radiant surface temperature may be less than the zone air temperature in heating mode, and greater than the zone air temperature in cooling mode, this simplifying assumption may tend to overestimate radiative exchange. Additional research is needed to refine calculation of equivalent combined surface coefficients that integrate radiative exchange with convective heat transfer of more advanced algorithms. The current simplified calculation results in the following:

- Resulting BESTEST-EX combined coefficients are 9\%-24\% lower than the ASHRAE combined coefficients

- A more realistic algorithm for surface convection is applied

- Reference simulation modeling is improved.

EnergyPlus simulations were performed to assess the equivalence of the "B-EX" constant inputs. Because of the nature of the inside heat balance formulation in EnergyPlus, constant combined coefficients could not be implemented. Therefore, the constant convective terms were used in simulations that automatically calculate interior radiative exchange, and compared to the full "detailed" interior convection algorithm simulations. The constant convective coefficients led to annual ideal sensible heating (Colorado Springs) and cooling (Las Vegas) loads 4.1\% and 4.8\% less, respectively, versus the "detailed" interior convection 
algorithm simulations (using Case L200EX nominal inputs, except with full-year heating/cooling seasons).

Table C1-1. Disaggregated Interior Surface Film Coefficients

$\left(\mathrm{Btu} /\left(\mathbf{h} \cdot \mathrm{ft}^{2} \cdot{ }^{\circ} \mathrm{F}\right)\right)$

\begin{tabular}{|c|c|c|}
\hline Vertical Surfaces $\left(T=68^{\circ} \mathrm{F}\right)\left(528^{\circ} \mathrm{R}\right)(\varepsilon=0.9)$ & B-EX & HERS \\
\hline$h_{i}$ & 0.908 & 0.908 \\
\hline$h_{c}$ & 0.305 & 0.552 \\
\hline$h_{s}=h_{i}+h_{c}$ & 1.213 & 1.460 \\
\hline \multicolumn{3}{|c|}{ Gables/Roof Surfaces $\left(T=68^{\circ} \mathrm{F}\right)\left(528^{\circ} \mathrm{R}\right)(\varepsilon=0.9)($ Note 1$)$} \\
\hline$h_{i}$ & 0.908 & 0.908 \\
\hline $\mathrm{h}_{\mathrm{c}}$ & 0.240 & 0.422 \\
\hline$h_{s}=h_{i}+h_{c}$ & 1.148 & 1.330 \\
\hline \multicolumn{3}{|l|}{ Horizontal Surfaces $\left(T=68^{\circ} \mathrm{F}\right)\left(528^{\circ} \mathrm{R}\right)(\varepsilon=0.9)$} \\
\hline$h_{i}$ & 0.908 & 0.908 \\
\hline$h_{c}$ & 0.255 & 0.399 \\
\hline$h_{s}=h_{i}+h_{c}$ & 1.163 & 1.307 \\
\hline \multicolumn{3}{|c|}{ Single-Pane Glass Surfaces $\left(T=68^{\circ} \mathrm{F}\right)\left(528^{\circ} \mathrm{R}\right)(\varepsilon=0.84)$} \\
\hline$h_{i}$ & 0.848 & 0.848 \\
\hline $\mathrm{h}_{\mathrm{c}}$ & 0.267 & 0.612 \\
\hline$h_{s}=h_{i}+h_{c}$ & 1.115 & 1.460 \\
\hline \multicolumn{3}{|c|}{ Single-Pane Window Aluminum Frame Surfaces $\left(T=68^{\circ} \mathrm{F}\right)\left(528^{\circ} \mathrm{R}\right)(\varepsilon=0.84)($ Note 2$)$} \\
\hline$h_{i}$ & 0.848 & 0.848 \\
\hline $\mathrm{h}_{\mathrm{c}}$ & 0.432 & 0.612 \\
\hline$h_{s}=h_{i}+h_{c}$ & 1.280 & 1.460 \\
\hline \multicolumn{3}{|c|}{ Low-e Glass Surfaces $\left(T=68^{\circ} \mathrm{F}\right)\left(528^{\circ} \mathrm{R}\right)(\varepsilon=0.84)$} \\
\hline$h_{i}$ & 0.848 & 0.848 \\
\hline$h_{c}$ & 0.222 & 0.485 \\
\hline$h_{s}=h_{i}+h_{c}$ & 1.070 & 1.333 \\
\hline \multicolumn{3}{|c|}{ Low-e Window Wood Frame Surfaces $\left(T=68^{\circ} \mathrm{F}\right)\left(528^{\circ} \mathrm{R}\right)(\varepsilon=0.84)$ (Note 2$)$} \\
\hline$h_{i}$ & 0.848 & 0.848 \\
\hline $\mathrm{h}_{\mathrm{c}}$ & 0.368 & 0.485 \\
\hline$h_{s}=h_{i}+h_{c}$ & 1.216 & 1.333 \\
\hline
\end{tabular}

Note 1: The values for HERS BESTEST sloped surface are shown here, though different coefficients would be used for the gable and roof surfaces types in HERS BESTEST.

Note 2: Window glass and frames are disaggregated here because overall air-air U-value for these components is greater than that for other envelope components. 


\section{C.1.1 Convective Portion}

The EnergyPlus "detailed" interior convection algorithm was used in the reference simulations. Unlike HERS BESTEST, which assigns constant convection coefficients for interior surfaces based on their orientation only, the EnergyPlus detailed algorithm used in the EnergyPlus reference simulations calculates the interior convection coefficients based on the temperature difference between the surface and zone air, as well as the orientation of the surface (interior convection coefficients for the window panes are calculated in EnergyPlus V3.1 following ISO 15099 Section 8.3.2.2, which is summarized in EnergyPlus Engineering Reference [2009]). According to the EnergyPlus Engineering Reference, the "detailed" algorithm is taken from Walton (1983), which is derived from Table 5 on p. 3.12 in the ASHRAE Handbook of Fundamentals (2001).

For vertical surfaces, the convection coefficient is calculated according to the equation

$$
h_{c}=1.31|\Delta T|^{\frac{1}{3}}
$$

where $\Delta T=\{($ Air Temperature $)-($ Surface Temperature $)\}(\mathrm{K})$ and $h_{c}$ is the interior convection coefficient having units of $\mathrm{W} /\left(\mathrm{m}^{2} \cdot \mathrm{K}\right)$.

For $(\Delta T<0.0$ AND an upward facing surface) OR $(\Delta T>0.0$ AND a downward facing surface $)$ the following relationship for enhanced convection is used:

$$
h_{c}=\frac{9.482|\Delta T|^{\frac{1}{3}}}{7.283-|\cos \Sigma|}
$$

where $\Sigma$ is the surface tilt angle.

For $(\Delta T>0.0$ AND an upward facing surface) $\mathrm{OR}(\Delta T<0.0$ AND a downward facing surface $)$ the following relationship for reduced convection is used:

$$
h_{c}=\frac{1.810|\Delta T|^{\frac{1}{3}}}{1.382+|\cos \Sigma|}
$$

EnergyPlus evaluates the interior convection coefficients on a time step basis. Therefore, because of the transient nature of the interior surface and zone air (when floating) temperatures, the interior convection coefficients change with time. To provide equivalent inputs for programs that require constant interior surface heat transfer coefficients, EnergyPlus simulations were performed and the time-varying interior convection coefficients were analyzed (using Case L200EX nominal inputs). For each surface type (exterior wall framed, exterior wall unframed, interior wall, door, floor framed, floor batting, ceiling framed, ceiling batting, attic floor framed, attic floor batting, gable, roof, window pane, window frame), the average interior convection coefficient was found for the core heating and cooling seasons. This was October-April for heating in Colorado Springs and May-September for cooling in Las Vegas. For each surface type, the Colorado Springs and Las Vegas values were then averaged. Equivalent constant convective surface coefficients were calculated using area weighting as shown in Tables C1-2 through C1-4; area-weighted averages are indicated with bold font in the tables. 
Table C1-2. Interior Convective Surface Coefficients for Vertical Surfaces

\begin{tabular}{|ccccc|}
\hline Surface & Area $\left(\mathbf{f t}^{\mathbf{2}}\right)$ & \multicolumn{3}{c|}{$\mathbf{h}_{\mathbf{c}}\left(\mathbf{B t u} / \mathbf{h} \cdot \mathbf{f t}^{2} \cdot{ }^{\mathbf{o}} \mathbf{F}\right)$} \\
& & Col Springs & Las Vegas & Avg \\
\hline Exterior Wall Framed & 258.5 & 0.305 & 0.315 & 0.310 \\
Exterior Wall Unframed & 775.5 & 0.341 & 0.316 & 0.329 \\
Interior Wall & 1024.0 & 0.272 & 0.295 & 0.284 \\
Door & 40.0 & 0.371 & 0.324 & 0.348 \\
Area-Weighted Average & & & & $\mathbf{0 . 3 0 5}$ \\
\hline
\end{tabular}

Table C1-3. Interior Convective Surface Coefficients for Roof and Gables

\begin{tabular}{|ccccc|}
\hline Surface & Area $\left(\mathbf{f t}^{2}\right)$ & \multicolumn{3}{c|}{$\mathbf{h}_{\mathbf{c}}\left(\mathbf{B t u} / \mathbf{h} \cdot \mathbf{f t}^{2} \cdot{ }^{\circ} \mathbf{F}\right)$} \\
& & Col Springs & Las Vegas & Avg \\
\hline Gable & 121.5 & 0.200 & 0.226 & 0.213 \\
Roof & 1622.2 & 0.255 & 0.228 & 0.242 \\
Area-Weighted Average & & & & $\mathbf{0 . 2 4 0}$ \\
\hline
\end{tabular}

Table C1-4. Interior Convective Surface Coefficients for Horizontal Surfaces

\begin{tabular}{|ccccc|}
\hline Surface & Area $\left(\mathbf{f t}^{\mathbf{2}}\right)$ & \multicolumn{3}{c|}{$\mathbf{h}_{\mathbf{c}}\left(\mathbf{B t u} / \mathbf{h} \cdot \mathbf{f t}^{\mathbf{2}} \cdot{ }^{\circ} \mathbf{F}\right)$} \\
& & Col Springs & Las Vegas & Avg \\
\hline Raised Floor & 1539.0 & 0.225 & 0.349 & 0.287 \\
Ceiling Framed & 153.9 & 0.343 & 0.197 & 0.270 \\
Ceiling Batting & 1385.1 & 0.299 & 0.185 & 0.242 \\
Attic Floor Framed & 153.9 & 0.341 & 0.254 & 0.297 \\
Attic Floor Batting & 1385.1 & 0.271 & 0.181 & 0.226 \\
Area-Weighted Average & & & & $\mathbf{0 . 2 5 5}$ \\
\hline
\end{tabular}

Disaggregated average values for the pane and frame (highlighted in bold below) were applied as shown in Table C1-5. Values for low-e windows were calculated similarly.

Table C1-5. Interior Convective Surface Coefficients for Single-Pane Windows

\begin{tabular}{|c|c|c|c|c|}
\hline \multirow[t]{2}{*}{ Surface } & \multirow[t]{2}{*}{ Area $\left(\mathrm{ft}^{2}\right)$} & \multicolumn{3}{|c|}{$h_{c}\left(B t u / h \cdot f^{2}{ }^{2}{ }^{\circ} F\right)$} \\
\hline & & Col Springs & Las Vegas & Avg \\
\hline Window Pane & 10.96 & 0.298 & 0.236 & 0.267 \\
\hline Window Frame & 4.04 & 0.475 & 0.389 & 0.432 \\
\hline
\end{tabular}

\section{C.1.1.1 Difference Between Surface Heat Transfer Convective Calculation in EnergyPlus} Version 4.0 Versus Version 3.1

After several simulation trials of the test specification were completed, an updated version of EnergyPlus (Version 4.0) was released. Documentation accompanying the release indicates the correction of an error in the EnergyPlus algorithm used to calculate convective heat transfer coefficients on the interior surfaces of windows. The correction has a secondary effect $(<0.3 \%)$ on the EnergyPlus calculation of the window exterior surface convective coefficients. The effect of the Version 3.1 error on EnergyPlus results is shown in Table C1-6. The effect of the error on average reference program base case energy use (L200EX-P) and window retrofit energy savings (L200-L250EX-P) is shown in Figures C1-1 and C1-2 for the physics heating and cooling cases, respectively. The DOE-2.1E and SUNREL reference results (used with EnergyPlus results to calculate average reference results) were generated by applying interior combined surface coefficients based on EnergyPlus versions 3.1 and 4.0, as shown for the labeled average 
results bars. In these figures the y-axes for the window retrofit sensitivity results are magnified relative to the y-axes for the base case energy use results.

Table C1-6. Effect of Window Interior Surface Convective Calculation for EnergyPlus Version 3.1 Versus Version 4.0

\begin{tabular}{|c|c|c|c|c|}
\hline EnergyPlus Version & $\begin{array}{l}\text { Version } \\
3.1\end{array}$ & $\begin{array}{l}\text { Version } \\
4.0\end{array}$ & $\begin{array}{l}\text { V3.1- } \\
\text { V4.0 }\end{array}$ & $\begin{array}{c}\text { \% Error } \\
([\mathrm{V} 3.1]-[\mathrm{V} 4.0]) /(\mathrm{V} 4.0)\end{array}$ \\
\hline \multicolumn{5}{|l|}{$\begin{array}{l}\text { Single-Pane Window Interior Surf. Coeffs. } \\
\left.\left(\text { Btu/(h-ft }{ }^{2} \cdot{ }^{\circ} \mathrm{F}\right)\right)\end{array}$} \\
\hline $\mathrm{h}_{\mathrm{c}}$ & 0.267 & 0.318 & -0.051 & $-16.0 \%$ \\
\hline$h_{s}=h_{i}+h_{c}$ & 1.115 & 1.166 & -0.051 & $-4.4 \%$ \\
\hline \multicolumn{5}{|l|}{$\begin{array}{l}\text { Low-e Window Interior Surface Coeffs. } \\
\left(\mathrm{Btu} /\left(\mathrm{h} \cdot \mathrm{ft}^{2} \cdot{ }^{\circ} \mathrm{F}\right)\right)\end{array}$} \\
\hline$h_{c}$ & 0.222 & 0.264 & -0.042 & $-15.9 \%$ \\
\hline$h_{s}=h_{i}+h_{c}$ & 1.070 & 1.112 & -0.042 & $-3.8 \%$ \\
\hline \multicolumn{5}{|l|}{ Annual Gas Use, with heating (MBtu/yr) } \\
\hline L200EX-P (base case) & 119.01 & 120.13 & -1.12 & $-0.9 \%$ \\
\hline $\begin{array}{l}\text { "L200EX-P" - “L250EX-P” } \\
\text { (single-pane v. low-e window sensitivity) }\end{array}$ & 10.86 & 11.85 & -0.99 & $-8.4 \%$ \\
\hline \multicolumn{5}{|l|}{ Annual Electric Use, with cooling (kWh/yr) } \\
\hline L200EX-P (base case) & 10665 & 10676 & -11 & $-0.1 \%$ \\
\hline $\begin{array}{l}\text { "L200EX-P" - “L250EX-P” } \\
\text { (single-pane v. low-e window sensitivity) }\end{array}$ & 1310 & 1316 & -6 & $-0.5 \%$ \\
\hline
\end{tabular}
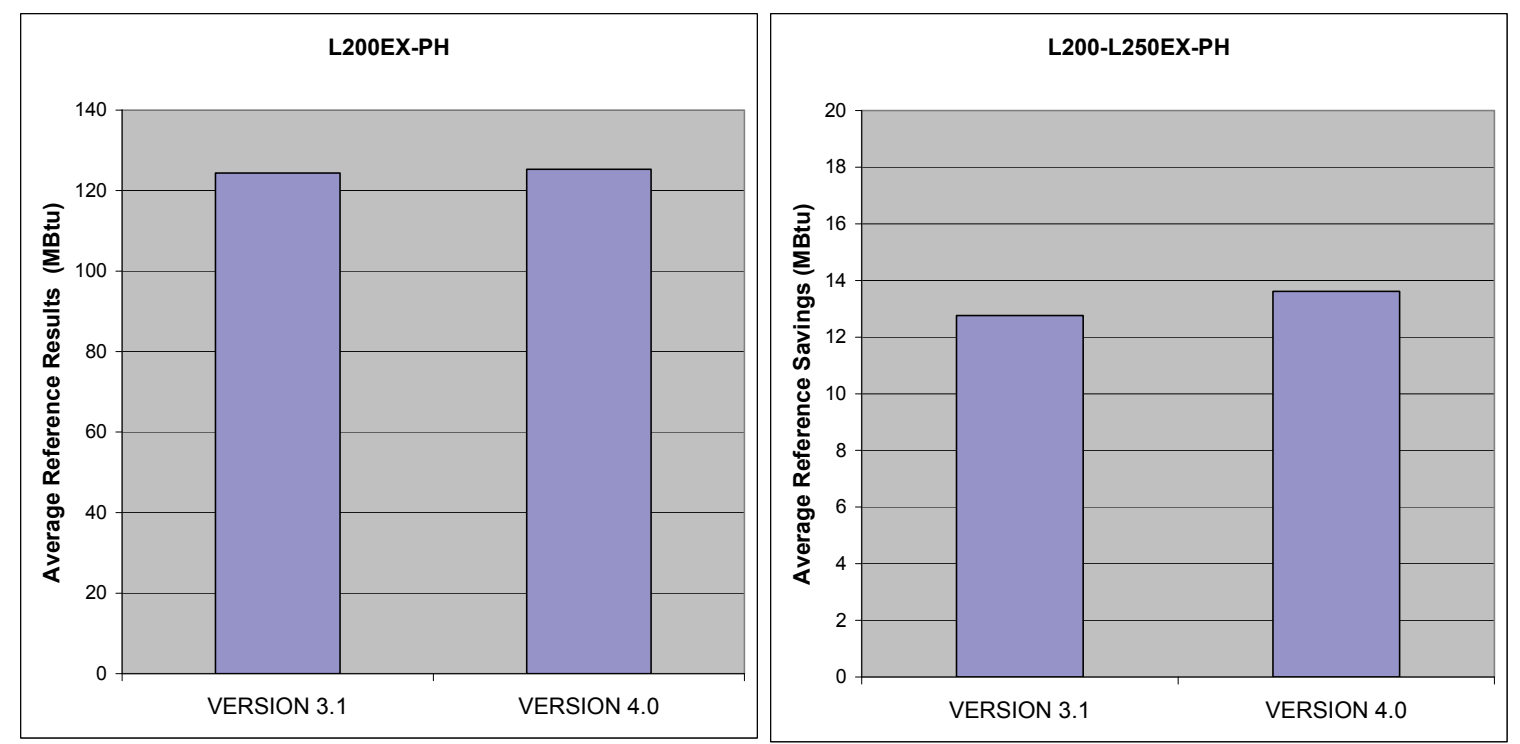

Figure C1-1. Effect of window interior convective surface coefficient on average reference results for gas use and savings in cases with space heating 

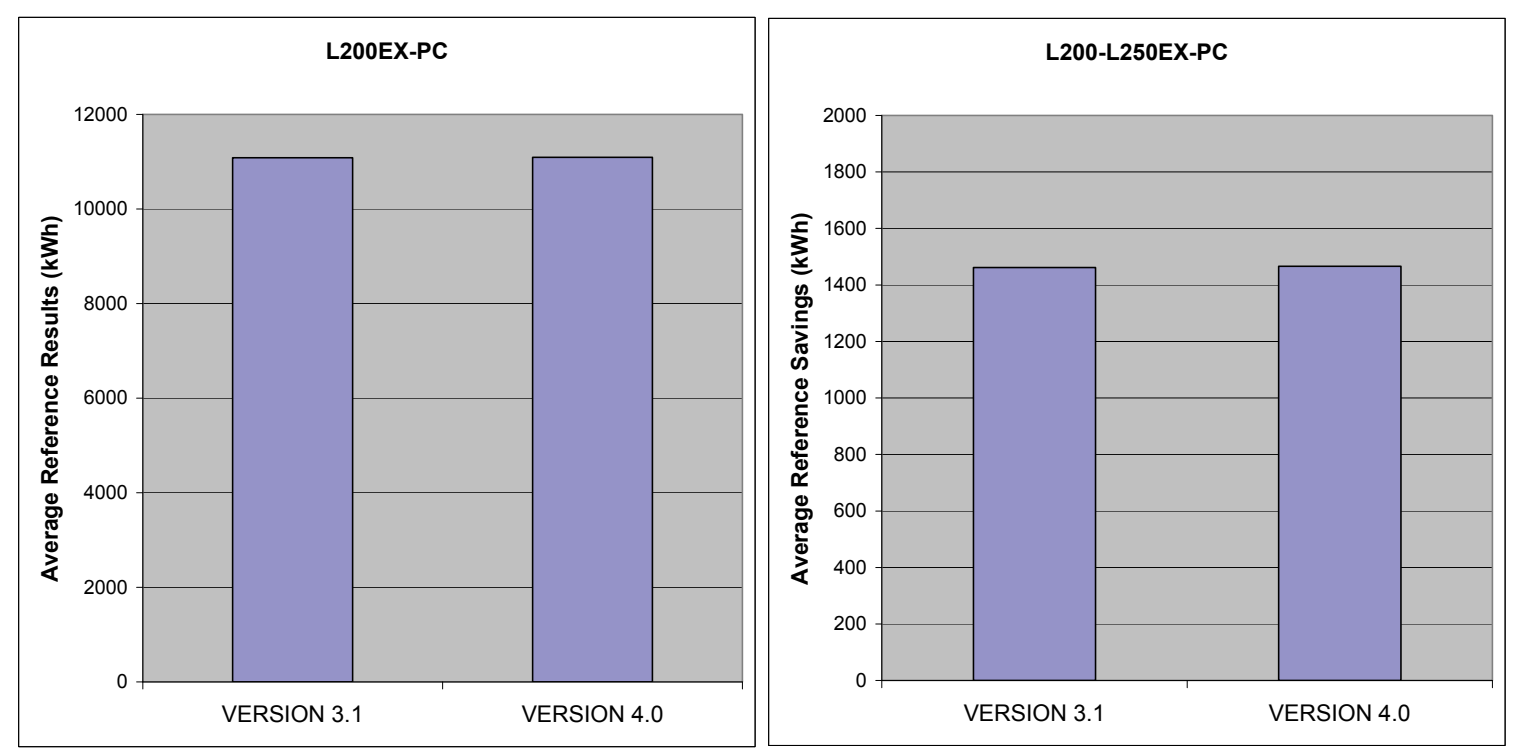

Figure C1-2. Effect of window interior convective surface coefficient on average reference results for electricity use and savings in cases with space cooling

The differences caused by the EnergyPlus version 3.1 error, shown in Figures C1-1 and C1-2, are relatively small compared to the range of disagreement among reference results shown in Appendix G, Section G.1. Because of project time constraints, these errors were not addressed for the initial version of BESTEST-EX. An update to a future version of BESTEST-EX is recommended to correct default combined interior surface coefficients for windows. Such a revision will also require re-running BESTEST-EX working group simulation trials and generating new reference utility billing data for the calibration tests. This update should be considered in parallel with other recommendations for future work described in Appendix I.

\section{C.1.2 Radiative Portion}

To provide equivalent interior combined surface coefficients, radiative portions must be assumed. The infrared portion of the film coefficients is based on the linearized gray-body radiation equation (Duffie and Beckman 1980)

$$
\mathrm{h}_{\mathrm{i}}=4 \varepsilon \sigma \mathrm{T}^{3}
$$

where:

$\varepsilon=$ infrared emissivity

$\sigma=0.1718 \times 10^{-8} \mathrm{Btu} /\left(\mathrm{h} \cdot \mathrm{ft}^{2} \cdot{ }^{\circ} \mathrm{F}\right)($ Stefan-Boltzmann constant $)$

$\mathrm{T}=$ average temperature of surrounding surfaces (assumed $50^{\circ} \mathrm{F}\left[510^{\circ} \mathrm{R}\right]$ for outside, $68^{\circ} \mathrm{F}\left[528^{\circ} \mathrm{R}\right]$ for inside)

$\mathrm{h}_{\mathrm{i}}=$ infrared radiation portion of surface coefficient 


\section{C.2 Exterior Surface Coefficients}

\section{C.2.1 Combined Heat Transfer Coefficients}

Using combined coefficients is a simplification of the convective and radiative heat transfer at the external surfaces. For example, there may be instances for a surface when there is a net energy loss through radiant exchange with the sky, but almost no temperature difference exists between the air and the surface or the ground and the surface. For situations such as these it is difficult to capture the physical behavior with one combined heat transfer coefficient that is multiplied by the surface-air temperature difference.

However, combined exterior heat transfer coefficients (provided for programs that do not automatically calculate surface heat transfer) were calculated using the method described in Appendix C of HERS BESTEST. One notable difference from HERS BESTEST is the use of corrected wind speeds at the surface centroids (see Section C.3) instead of the meteorological wind speed from the weather file.

First the time-average meteorological wind speeds were calculated for core heating and cooling seasons. This is October-April for heating in Colorado Springs and May-September for cooling in Las Vegas. The "core average" wind speeds are $4.44 \mathrm{~m} / \mathrm{s}(9.93 \mathrm{mph})$ and $4.28 \mathrm{~m} / \mathrm{s}(9.57 \mathrm{mph})$, respectively. The wind speeds were then corrected (see Section C.3) and used to calculate the exterior combined radiative and convective surface coefficients, $h_{o}$, according to

$$
h_{o}=a_{1}+a_{2} V+a_{3} V^{2}
$$

which is a second order polynomial in corrected wind speed $V$ having coefficients $a_{i}$ (see HERS BESTEST Appendix C) that depend on the surface texture.

Similar to the methodology used in HERS BESTEST (Appendix C), a brick or rough plaster surface texture was assumed for the raised floor, exterior walls, doors, gables, and roofs when calculating the coefficients of Equation C-5. In HERS BESTEST the exterior surface coefficients for the windows were calculated based on analysis using the program WINDOW 4.1 (1994). For BESTEST-EX the equivalent combined coefficients for the windows were calculated using Equation C-5, assuming a very smooth surface. The results of these calculations are summarized in Table C2-1.

Table C2-1 can be further simplified to the area-weighted average (see Section C2.2 for description of area weighting) external heat transfer coefficients shown in Table C2-2.

Table C2-1. Combined Exterior Heat Transfer Coefficients for Each Surface Type

\begin{tabular}{|c|c|c|c|c|c|}
\hline Surface & Height (ft) & $\begin{array}{l}\text { Wind Speed } \\
\text { Corr. Factor }\end{array}$ & $\begin{array}{c}\text { CO Ext Surf } \\
\text { Coeff } \\
\left(B t u /\left(h \cdot f^{2} \cdot{ }^{\circ} F\right)\right)\end{array}$ & 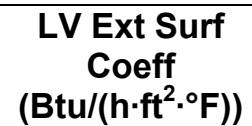 & $\begin{array}{l}\text { Avg. Ext Surf Coeff } \\
\left(\text { Btu } /\left(\mathrm{h} \cdot \mathrm{ft}^{2} \cdot{ }^{\circ} \mathrm{F}\right)\right)\end{array}$ \\
\hline Floor & 0.000 & 0.000 & 2.200 & 2.200 & 2.200 \\
\hline Exterior Walls & 4.000 & 0.451 & 3.656 & 3.603 & 3.630 \\
\hline Windows (pane) & 4.500 & 0.463 & 2.630 & 2.588 & 2.609 \\
\hline Windows (frame) & 4.500 & 0.463 & 2.630 & 2.588 & 2.609 \\
\hline Doors & 3.333 & 0.433 & 3.598 & 3.547 & 3.573 \\
\hline Gables & 9.500 & 0.546 & 3.966 & 3.902 & 3.934 \\
\hline Roof & 10.250 & 0.555 & 3.997 & 3.931 & 3.964 \\
\hline
\end{tabular}


Table C2-2. Combined Exterior Heat Transfer Coefficients After Area Weighting

\begin{tabular}{|c|c|}
\hline Surface & $\boldsymbol{h}_{\mathbf{o}}\left(\mathrm{Btu} /\left(\mathbf{h} \cdot \mathbf{f t}^{2} \cdot{ }^{\circ} \mathrm{F}\right)\right)$ \\
\hline Floor & 2.200 \\
Exterior Walls/Doors & 3.628 \\
Gables/Roof & 3.962 \\
Window & 2.609 \\
\hline
\end{tabular}

\section{C.2.2 Disaggregation of Combined Coefficients Into Convective and Radiative Components}

The combined exterior surface coefficients presented in Table C2-2 are disaggregated in Table C2-3 using the two different methods outlined below:

1. "B-EX1" as described in Section C.2.2.1.

2. "B-EX2" as described in Section C.2.2.2.

A simplifying assumption for disaggregating convective and radiative portions of combined surface coefficients is that for the radiative portion for a given surface, the mean radiant temperature of the sky and other surrounding surfaces and objects equals the ambient air temperature. The actual mean radiant temperature is likely to differ from the ambient temperature. Additional research to refine calculation of equivalent combined surface coefficients that integrate radiative exchange with convective heat transfer of more advanced algorithms is needed. Such research should also consider developing future exterior surface coefficients based on the additive method for developing interior surface coefficients applied in Section C.1.

If the program being tested requires use of disaggregated surface coefficients, either the "B-EX1" or "BEX2" coefficients may be used. Other disaggregated coefficients may also be used if there is a logical basis for selecting them. For historical reference, the original HERS BESTEST coefficients are also shown in italics under the heading "HERS."

A sensitivity test comparing the use of combined surface coefficients to more detailed surface heat transfer modeling was done with EnergyPlus. EnergyPlus simulations (using Case L200EX nominal inputs, except with full-year heating/cooling seasons) with the constant combined exterior surface coefficients with detailed calculation of radiation exchange disabled result in a Colorado Springs annual heating load that was $6.2 \%$ greater than the more detailed simulation using EnergyPlus's "DOE2" exterior surface heat transfer algorithm. For Las Vegas, using the constant combined exterior surface coefficients results in a cooling load that agrees within $1 \%$ versus the more detailed simulation. The combined coefficients have better agreement with EnergyPlus's "simple" model than its "DOE2" model. 
Table C2-3. Disaggregated Exterior Film Coefficients for Opaque Surfaces $\left(\mathrm{Btu} /\left(\mathbf{h} \cdot \mathrm{ft}^{2} \cdot{ }^{\circ} \mathrm{F}\right)\right)$

\begin{tabular}{|c|c|c|c|}
\hline Exterior Walls/Doors, Outside $\left(\mathrm{T}=50^{\circ} \mathrm{F}\right)\left(510^{\circ} \mathrm{R}\right)(\varepsilon=0.9)$ & B-EX1 & B-EX2 & HERS \\
\hline$h_{i}=h_{o}-h_{c}$ for "B-EX1" & 2.452 & 0.819 & 0.819 \\
\hline$h_{c}=h_{o}-h_{i}$ for "B-EX2" & 1.176 & 2.809 & 4.929 \\
\hline$h_{\circ}$ & 3.628 & 3.628 & 5.748 \\
\hline \multicolumn{4}{|l|}{ Gables/Roof, Outside $\left(T=50^{\circ} \mathrm{F}\right)\left(510^{\circ} \mathrm{R}\right)(\varepsilon=0.9)$} \\
\hline$h_{i}=h_{o}-h_{c}$ for "B-EX1" & 2.574 & 0.819 & 0.819 \\
\hline$h_{c}=h_{o}-h_{i}$ for "B-EX2" & 1.388 & 3.143 & 4.929 \\
\hline$h_{0}$ & 3.962 & 3.962 & 5.748 \\
\hline \multicolumn{4}{|l|}{ Raised Floor, Outside $\left(T=50^{\circ} \mathrm{F}\right)\left(510^{\circ} \mathrm{R}\right)(\varepsilon=0.9)$} \\
\hline$h_{i}=h_{o}-h_{c}$ for "B-EX1" & 2.068 & 0.819 & 0.819 \\
\hline$h_{c}=h_{o}-h_{i}$ for "B-EX2" & 0.132 & 1.381 & 1.381 \\
\hline $\mathrm{h}_{\mathrm{o}}$ & 2.200 & 2.200 & 2.200 \\
\hline & \multicolumn{3}{|c|}{ All Types of Windows } \\
\hline Windows: Very Smooth Surface Outside $\left(T=50^{\circ} \mathrm{F}\right)\left(510^{\circ} \mathrm{R}\right)(\varepsilon=0.84)$ & B-EX1 & B-EX2 & HERS \\
\hline$h_{i}=h_{o}-h_{c}$ for "B-EX1" & 1.750 & 0.764 & 0.764 \\
\hline$h_{c}=h_{o}-h_{i}$ for "B-EX2" & 0.859 & 1.845 & 3.492 \\
\hline $\mathrm{h}_{\mathrm{o}}$ & 2.609 & 2.609 & 4.256 \\
\hline
\end{tabular}

\section{C.2.2.1 Disaggregation Based on Calculated Convective Portion by EnergyPlus}

One way to disaggregate the combined surface coefficients, $h_{o}$, into convective and radiative components is to estimate the convective component, $h_{c}$. The radiative component $h_{i}$ is then $h_{i}=h_{o}-h_{c}$. The coefficients calculated using this method are labeled "B-EX1" in Table C2-3.

The "DOE2" exterior convection algorithm was used in EnergyPlus to generate reference results. According to EnergyPlus documentation, the DOE2 algorithm is a combination of the MoWiTT (Yazdanian and Klems 1994) and BLAST detailed algorithms. The "DOE2" algorithm improves on the simplified HERS BESTEST methodology (HERS BESTEST vol. 1, Appendices C and D), in which the combined exterior heat transfer coefficients are calculated based on the surface texture and meteorological wind speed. Instead, the "DOE2" algorithm uses a corrected wind speed (see Section C.3) and incorporates temperature-dependent natural convection (see Section C.1) in addition to wind-driven convection. Radiant exchange between the surface and the ground, sky, and air is modeled separately.

For each surface the exterior convection coefficient, $h_{c, \text { glass }}$ (a very smooth glass surface is initially assumed) is calculated using the relationship

$$
h_{c, \text { glass }}=\sqrt{h_{n}^{2}+\left(a V_{z}^{b}\right)^{2}}
$$


where $h_{n}$ is the natural convection coefficient $\left(\mathrm{W} / \mathrm{m}^{2} \cdot \mathrm{K}\right)$ calculated using the same methodology as the interior convection coefficients (See Section C.1). $V_{z}$ is the wind speed $(\mathrm{m} / \mathrm{s}$ ) at the height of the centroid of the surface above ground (See Section C.3 for a description of the wind speed correction). The constants $\mathrm{a}$ and $\mathrm{b}$ depend on whether the surface is windward $(\mathrm{a}=2.38, \mathrm{~b}=0.89)$ or leeward $(\mathrm{a}=2.86$, $\mathrm{b}=0.617$ ). Any surface more than 100 degrees from normal incidence is considered leeward.

The coefficient $h_{c, \text { glass }}$ is then corrected based on the roughness of the surface using the equation

$$
h_{c}=h_{n}+R_{f}\left(h_{c, \text { glass }}-h_{n}\right) \quad(\mathrm{C}-7)
$$

where $R_{f}$ is surface roughness multiplier chosen from Table C2-4.

Table C2-4. Surface Roughness Multipliers

\begin{tabular}{|c|c|}
\hline Roughness Index & $\boldsymbol{R}_{\boldsymbol{f}}$ \\
\hline Very Rough & 2.17 \\
\hline Rough & 1.67 \\
\hline Medium Rough & 1.52 \\
\hline Medium Smooth & 1.13 \\
\hline Smooth & 1.11 \\
\hline Very Smooth & 1.00 \\
\hline
\end{tabular}

Because wind direction, wind speed, surface temperature, and outdoor temperature all change with time, the exterior convection coefficients in the EnergyPlus reference simulations vary with time. To develop example equivalent inputs for those programs that require constant disaggregated convective and radiative exterior surface heat transfer coefficients, EnergyPlus simulations using Case L200EX nominal inputs were performed and the time-varying exterior convection coefficients output by EnergyPlus were analyzed. For each surface type (exterior wall framed, exterior wall unframed, door, roof, gable, window pane, window frame, floor framed, and floor batting), the average exterior convection coefficient was found during the core heating and cooling seasons. This is October-April for heating in Colorado Springs and May-September for cooling in Las Vegas. For each surface type, the Colorado Springs and Las Vegas values were then averaged. Equivalent constant convective coefficients were calculated using area weighting as shown in Tables C2-5 through C2-8.

Table C2-5. Exterior Convective Surface Coefficients for Vertical Surfaces

\begin{tabular}{|ccccc|}
\hline Surface & Area $\mathbf{( f t}^{\mathbf{2}} \mathbf{)}$ & Col Springs & $\begin{array}{c}\mathbf{h}_{\mathbf{c}}\left(\mathbf{B t u} /\left(\mathbf{h} \cdot \mathbf{f t}^{\mathbf{2}} \cdot{ }^{\circ} \mathbf{F}\right)\right) \\
\text { Las Vegas }\end{array}$ & Average \\
\hline Ext Wall Framed & & 1.191 & 1.163 & 1.177 \\
Ext Wall Unframed & 258.5 & 1.192 & 1.163 & 1.177 \\
Door & 475.5 & 1.153 & 1.121 & 1.137 \\
Area-Weighted Average & & & & $\mathbf{1 . 1 7 6}$ \\
\hline
\end{tabular}

Table C2-6. Exterior Convective Surface Coefficients for Roof and Gables

\begin{tabular}{|ccccc|}
\hline Surface & Area $\left(\mathbf{f t}^{2}\right)$ & \multicolumn{3}{c|}{$\mathbf{h}_{\mathbf{c}}\left(\mathbf{B t u} /\left(\mathbf{h} \cdot \mathbf{f t}^{\mathbf{2}} \cdot{ }^{\circ} \mathbf{F}\right)\right)$} \\
& & Col Springs & Las Vegas & Average \\
\hline Roof & 1622.2 & 1.391 & 1.387 & 1.389 \\
Gables & 121.5 & 1.387 & 1.354 & 1.371 \\
Area-Weighted Average & & & & $\mathbf{1 . 3 8 8}$ \\
\hline
\end{tabular}


Table C2-7. Exterior Convective Surface Coefficients for the Floor

\begin{tabular}{|ccccc|}
\hline Surface & Area $\left(\mathbf{f t t}^{\mathbf{2}}\right)$ & \multicolumn{3}{c|}{$\mathbf{h}_{\mathbf{c}}\left(\mathbf{B t u} /\left(\mathbf{h} \cdot \mathbf{f t}^{2} \cdot{ }^{\circ} \mathbf{F}\right)\right)$} \\
& & Col Springs & Las Vegas & Average \\
\hline Floor Framed & 153.9 & 0.205 & 0.187 & 0.196 \\
Floor Batting & 1385.1 & 0.125 & 0.126 & 0.125 \\
Area-Weighted Average & & & & $\mathbf{0 . 1 3 2}$ \\
\hline
\end{tabular}

Table C2-8. Exterior Convective Surface Coefficients for Windows

\begin{tabular}{|ccccc|}
\hline Surface & Area $\left(\mathbf{f t}^{\mathbf{2}}\right)$ & \multicolumn{3}{c|}{$\mathbf{h}_{\mathbf{c}}\left(\mathbf{B t u} /\left(\mathbf{h} \cdot \mathbf{f t}^{2} \cdot{ }^{\circ} \mathbf{F}\right)\right)$} \\
& & Col Springs & Las Vegas & Average \\
\hline Window Pane & 10.96 & 0.894 & 0.810 & 0.852 \\
Window Frame & 4.04 & 0.923 & 0.833 & 0.878 \\
Area-Weighted Average & & & & $\mathbf{0 . 8 5 9}$ \\
\hline
\end{tabular}

To verify the equivalence of these inputs, the constant exterior convection coefficient values were used in EnergyPlus simulations that automatically calculate radiative exchange and compared to the more detailed EnergyPlus simulations using the "DOE2" exterior surface heat transfer algorithm (using Case L200EX nominal inputs, except for full-year heating/cooling seasons). Results for the annual heating and cooling loads in Colorado Springs and Las Vegas, respectively, agreed within 2\%.

\section{C.2.2.2 Disaggregation Based on Calculated Radiative Portion Using HERS BESTEST Appendix D}

Another method to disaggregate the combined coefficients, $h_{o}$, is to estimate radiative coefficients $h_{i}$. The convective coefficients, $h_{c}$, are then the difference $h_{c}=h_{o}-h_{i}$. The coefficients calculated using this approach are labeled "B-EX2" in Table C2-3. Similar to Appendix D of HERS BESTEST, the infrared portion of the film coefficients is based on the linearized gray-body radiation equation (Duffie and Beckman 1980).

$$
\mathrm{h}_{\mathrm{i}}=4 \varepsilon \sigma \mathrm{T}^{3} \quad(\mathrm{C}-4)
$$

where:

$\varepsilon=$ infrared emissivity

$\sigma=0.1718 \times 10^{-8} \mathrm{Btu} /\left(\mathrm{h} \cdot \mathrm{ft}^{2} \cdot{ }^{\circ} \mathrm{F}\right)($ Stefan-Boltzmann constant $)$

$\mathrm{T}=$ average temperature of surrounding surfaces (assumed $50^{\circ} \mathrm{F}\left[510^{\circ} \mathrm{R}\right]$ for outside, $68^{\circ} \mathrm{F}\left[528^{\circ} \mathrm{R}\right]$ for inside)

$\mathrm{h}_{\mathrm{i}}=$ infrared radiation portion of surface coefficient

Other nomenclature used for Table C2-3 is:

$\mathrm{h}_{\mathrm{c}}=$ convective portion of surface coefficient

$\mathrm{h}_{\mathrm{o}}=$ total combined outside surface coefficient

\section{C.3 Wind Speed Correction}

In HERS BESTEST the equivalent exterior surface coefficients for the exterior walls, doors, and roofs are calculated using the mean annual weather station wind speed of $10.7 \mathrm{mph}$. The analysis using WINDOW 4.1 assumes a slightly lower value of $9.0 \mathrm{mph}$. In reality, the wind speed over the surfaces of a building could be considerably lower than wind speed from the weather file. For the BESTEST-EX reference simulations, EnergyPlus converts the weather station wind speed to the local wind speed at the centroid of each surface according to the equation (ASHRAE 2001, F16.3) 


$$
V=V_{\text {met }}\left(\frac{\delta_{\text {met }}}{z_{\text {met }}}\right)^{a-m e t}\left(\frac{z}{\delta}\right)^{a}
$$

where $V$ is the local wind speed, $V_{\text {met }}$ is the wind speed at the weather station, $z_{\text {met }}$ is the height of the wind speed measurement (assumed to be $10 \mathrm{~m}$ ), and $z$ is the height of the surface centroid. $\delta$ and $a$ are terraindependent coefficients found in ASHRAE (2001). The weather measurement is assumed to be in "flat, open country" ( $\delta=270 \mathrm{~m}, a=0.14$ ) and the site is assumed to be "suburbs" ( $\delta=370 \mathrm{~m}, a=0.22$ ). According to ASHRAE (2001), the descriptions for these terrain conditions are:

Flat, open country: "Open terrain with scattered obstruction having heights generally less than $10 \mathrm{~m}$, including flat open country typical of meteorological station surroundings."

Suburbs: "Urban and suburban areas, wooded areas, or other terrain with numerous closely spaced obstructions having the size of single-family dwellings or larger, over a distance of at least $2000 \mathrm{~m}$ or 10 times the height of the structure upwind, which ever is greater."

Equation C-8 is valid for an isolated building only. When the spacing-to-height values do not satisfy the terrain descriptions, the interference and shielding effects of nearby obstructions must be considered. As an example, the exterior walls of the conditioned zone in the BESTEST-EX model have a centroid height of approximately $1.22 \mathrm{~m}$. Applying Equation $\mathrm{C}-8$ gives

$$
V \approx 0.451\left(V_{m e t}\right)
$$




\section{Appendix D Infiltration Modeling}

Infiltration caused by envelope leakage in residential buildings significantly affects the annual heating and cooling loads. Multiple methods exist for modeling infiltration given the results of blower door tests. Examples of methods and models are discussed in the following sections.

\section{D.1 Conversion of $\mathrm{ACH}_{50}$ to Natural Air Changes per Hour}

Some programs may use a rule-of-thumb conversion of $\mathrm{ACH}_{50}$ to natural air changes per hour. For example, one common approach is to use the approximation developed by Kronvall and Persily (Sherman 1998).

$$
A C H \approx \frac{A C H_{50}}{20}
$$

Assuming this simple relationship, the nominal constant equivalent $\mathrm{ACH}$ input for the pre-retrofit basecase building (Case L200EX) would be 0.975 ACH. However, an EnergyPlus simulation using nominal inputs and $0.975 \mathrm{ACH}$ constant infiltration over-predicted the annual heating load in Colorado Springs by about $8 \%$. This suggests that the factor in the denominator of Equation D-1 may need to be modified based on the characteristics of the climate and building (for example, see Sherman 1987).

\section{D.2 LBL Model (Sherman and Grimsrud)}

The "Basic Model" described in the 2005 ASHRAE Handbook of Fundamentals (p. 27.21) was used in EnergyPlus to model infiltration. This model is based on the LBL infiltration model developed by Sherman and Grimsrud in 1980. The infiltration airflow rate is calculated using the relation

$$
Q=A_{L-4 P a} \sqrt{C_{s} \Delta t+C_{w} V_{m e t}^{2}}
$$

where $Q$ is the airflow rate in CFM, $A_{L-4 P a}$ is the effective leakage area at $4 \mathrm{~Pa}, C_{s}$ is the stack coefficient, $\Delta t$ is the indoor-outdoor temperature difference (absolute value, ${ }^{\circ} \mathrm{F}$ ), $C_{w}$ is the wind coefficient, and $V_{m e t}$ is the wind speed measured at the weather station (mph). $C_{s}$ and $C_{w}$ depend on the distribution of leakage area in the house, height/terrain at the site/weather station, and shelter class of the building. The following assumptions were made (see 2005 ASHRAE Handbook of Fundamentals, p. 27.21):

- Half of building leakage in walls

- Equal leakage in floor and ceiling

- One-story building ( $8 \mathrm{ft}$ or $2.5 \mathrm{~m})$

- Stack coefficient $\left(C_{s}\right)=0.0150 \mathrm{cfm}^{2} /\left(\right.$ in..$\left.^{4}{ }^{\circ} \mathrm{F}\right)$, or in SI units as input into EnergyPlus: $C_{s}=0.000145(\mathrm{~L} / \mathrm{s})^{2} /\left(\mathrm{cm}^{4} \cdot \mathrm{K}\right)$ corresponding with one-story house

- Wind coefficient $\left(C_{w}\right)=0.0012 \mathrm{cfm}^{2} /\left(\mathrm{in}^{4} \cdot \mathrm{mph}^{2}\right)$, or in SI units as input into EnergyPlus: $C_{w}=0.000032(\mathrm{~L} / \mathrm{s})^{2} /\left(\mathrm{cm}^{4}(\mathrm{~m} / \mathrm{s})^{2}\right)$ corresponding with:

- "Terrain used for converting meteorological wind speed is that of a rural area with scattered obstacles" 
- Shelter Class 5, "Typical shelter produced by buildings or other structures that are immediately adjacent (closer than one house height): e.g., neighboring houses on the same side of the street, trees, bushes, etc."

O One story house.

- $\quad$ Effective leakage area $\left(A_{L-4 P a}\right)=196.3$ in. $^{2}$, as documented below.

The effective leakage area at $4 \mathrm{~Pa}$ was calculated based on the Colorado Springs $\mathrm{CFM}_{50}$ nominal input $\left(4000 \mathrm{CFM}_{50}\right)$. First, the leakage area at $50 \mathrm{~Pa}$ was calculated using the equation (2005 ASHRAE Handbook of Fundamentals, p. 27.12)

$$
\left(A_{L-50 P a}\right)=B \frac{C F M_{50}}{C_{D}} \sqrt{\frac{\rho}{2 \Delta p_{r}}}
$$

where $B$ is a unit conversion factor $(0.186), \mathrm{CFM}_{50}$ is the airflow rate measured in CFM at $50 \mathrm{~Pa}, C_{D}$ is the discharge coefficient (assumed to be 1.0), $\rho$ is the density of air $\left(0.060 \mathrm{lbm} / \mathrm{ft}^{3}\right.$ for $6171 \mathrm{ft}$ altitude of Colorado Springs, using the equation of HERS BESTEST, Appendix B; for software that automatically calculates air density, the air density may have a different value) and $\Delta p_{r}$ is the reference pressure in inches of water $\left(50 \mathrm{~Pa} \times 1\right.$ in $\mathrm{H}_{2} \mathrm{O}$ per $\left.249 \mathrm{~Pa}\right)$. Next, the leakage area at $50 \mathrm{~Pa}$ was converted to a leakage area at $4 \mathrm{~Pa}$ using the relationship (2005 ASHRAE Handbook of Fundamentals, p. 27.13)

$$
A_{L-4 P a}=A_{L-50 P a}\left(\frac{C_{D-50 P a}}{C_{D-4 P a}}\right)\left(\frac{\Delta p_{r-4 P a}}{\Delta p_{r-50 P a}}\right)^{n-0.5}
$$

where $n$ is the pressure exponent (assumed to be 0.65 ) and the flow coefficient at $4 \mathrm{~Pa}$ is also equal to 1.0. Using Equations D-3 and D-4, the effective leakage area at $4 \mathrm{~Pa}$ is: $A_{L-4 P a}=196.3$ in. ${ }^{2}$.

Applying the Las Vegas altitude of $2178 \mathrm{ft}$ to adjust air density, and back-calculating based on $A_{L-4 P a}=$ 196.3 in. $^{2}$, yield an equivalent $3714 \mathrm{CFM}_{50}$ for Las Vegas.

Other Models

Software programs may use other infiltration models, such as AIM-2 (Walker and Wilson 1998) or a modified version of the two approaches discussed above.

\section{D.3 Equivalent Constant Infiltration Rates}

Equivalent constant infiltration rates are provided for programs that do not automatically calculate infiltration. Constant infiltration rates are based on the average of hourly weather-driven infiltration rates calculated by EnergyPlus over the core heating and cooling seasons for the Colorado Springs and Las Vegas climates, respectively; these are labeled as "E+ ACH" in Table D1-1. In this table, EnergyPlus simulations using its application of the Sherman-Grimsrud model (column labeled "S-G Load") are compared with EnergyPlus simulations using the equivalent constant infiltration rate (column labeled "E+ACHload").

\begin{tabular}{|c|c|c|c|c|c|c|c|c|}
\hline \multirow[b]{2}{*}{ ELA (in. ${ }^{2}$ ) } & \multicolumn{4}{|c|}{ Las Vegas } & \multicolumn{4}{|c|}{ Colorado Springs } \\
\hline & CFM50 & $\begin{array}{l}\text { S-G Load } \\
\text { (MBtu) }\end{array}$ & $\mathrm{E}+\mathrm{ACH}$ & $\begin{array}{c}\text { E+ACHload } \\
\text { (MBtu) }\end{array}$ & CFM50 & $\begin{array}{c}\text { S-G Load } \\
\text { (MBtu) }\end{array}$ & $\mathrm{E}+\mathrm{ACH}$ & $\begin{array}{c}\text { E+ACHload } \\
\text { (MBtu) }\end{array}$ \\
\hline 98.1 & 1857 & 58.0 & 0.246 & 57.7 & 2000 & 60.1 & 0.382 & 59.5 \\
\hline 137.4 & 2600 & 58.3 & 0.345 & 57.9 & 2800 & 65.2 & 0.534 & 64.4 \\
\hline 196.3 & 3714 & 58.9 & 0.492 & 58.2 & 4000 & 73.0 & 0.760 & 71.7 \\
\hline 215.9 & 4085 & 59.1 & 0.540 & 58.4 & 4400 & 75.5 & 0.835 & 74.2 \\
\hline
\end{tabular}

Table D1-1. EnergyPlus Infiltration Sensitivity Test Results 
Table D1-1 indicates 1\%-2\% agreement for use of constant values versus the detailed modeling. This table also indicates that infiltration has a greater effect on sensible heating load in Colorado Springs than on sensible cooling load in Las Vegas; this is because much of the infiltration is temperature-difference driven in the model.

\section{D.4 Comparison of Predicted Infiltration With Measured Infiltration}

Sensitivity tests were performed for heating load versus CFM-50 blower door measurements using the "Effective Leakage Area" model in EnergyPlus, which is based on the ASHRAE "Basic" residential infiltration model. Using the most sheltered assumption (Shelter Class 5), the savings appear to be about 0.065 therms load/(CFM-50 reduction) for an ideal space heating system or 0.092 therms use/(CFM-50 reduction) assuming a 70\% furnace efficiency. These values are above the high end of estimates of 0.050-0.060 therms use/(CFM-50 reduction) based on pre- and post-retrofit utility billing data (Blasnik 2009). Further research is needed to investigate the causes of these differences and if other models may yield better agreement with measured field data. 


\section{Appendix E Window Modeling with WINDOW 5}

WINDOW 5.2 (2005) was used to help develop thermal and optical property specifications for the following windows used in BESTEST-EX:

- Clear single-pane glass with aluminum frame with thermal break (see Section E.1)

- Double-pane low-e glass with wood frame (see Section E.2).

\section{E.1 Single-Pane Window With Aluminum Frame With Thermal Break, Output From WINDOW 5}

The WINDOW 5 output listing below includes analysis results and inputs for the BESTEST-EX basecase (L200EX) single-pane window. The following inputs applied in the analysis are not listed:

- "NFRC" calculation mode is applied

- Environmental conditions are identical to "NFRC 100-2001", except:

○ Fixed combined interior surface coefficient $=1.115 \mathrm{Btu} /\left(\mathrm{h} \cdot \mathrm{ft}^{2} \cdot{ }^{\circ} \mathrm{F}\right)$

$\circ$ Fixed combined exterior surface coefficient $=2.609 \mathrm{Btu} /\left(\mathrm{h} \cdot \mathrm{ft}^{2} \cdot{ }^{\circ} \mathrm{F}\right)$.

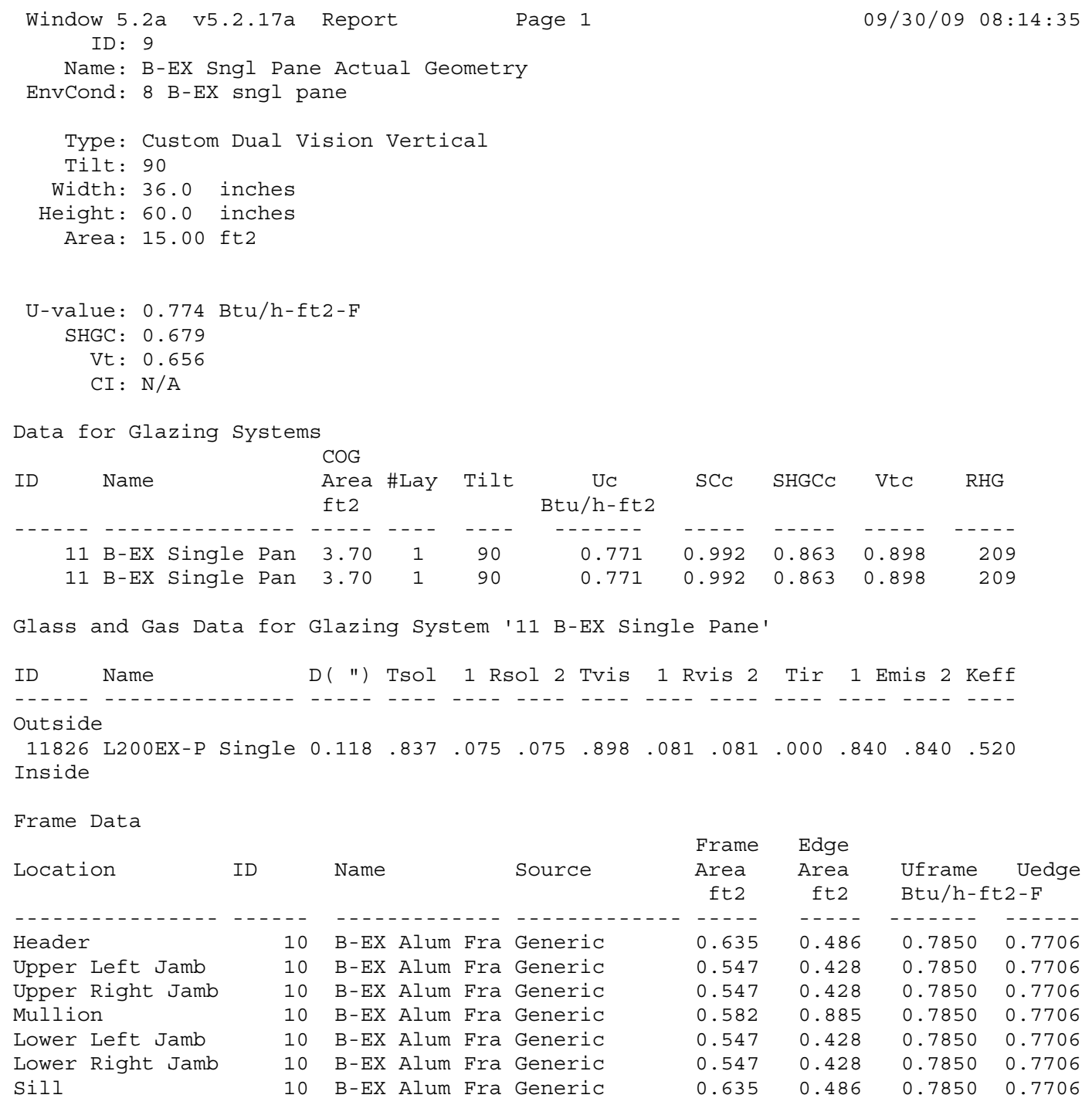


Gas Data

ID Name

\begin{tabular}{|c|c|c|c|c|c|}
\hline Type & $\begin{array}{l}\text { Cond } \\
\text { Btu/h- } \\
\text { ft-F }\end{array}$ & $\begin{array}{c}\text { Visc } \\
\text { lb/ft- } \\
\text { s } \\
x e-6\end{array}$ & $\begin{array}{c}\mathrm{Cp} \\
\text { Btu/1b- } \\
\text { F }\end{array}$ & $\begin{array}{c}\text { Dens } \\
1 \mathrm{~b} / \mathrm{ft} 3\end{array}$ & Pran \\
\hline
\end{tabular}

No gas data for Single Glazing

Window 5.2a v5.2.17a Report

Page 2

$09 / 30 / 0908: 14: 35$

Environmental Conditions: 8 B-EX sngl pane

\begin{tabular}{|c|c|c|c|c|c|c|c|}
\hline & $\begin{array}{l}\text { Tout } \\
\text { (F) }\end{array}$ & $\begin{array}{l}\operatorname{Tin} \\
(\mathrm{F})\end{array}$ & $\begin{array}{l}\text { WndSpd } \\
\text { (mph) }\end{array}$ & Wnd Dir & $\begin{array}{l}\text { Solar } \\
\text { (Btu/h- }\end{array}$ & $\begin{array}{l}\text { Tsky } \\
\text { Et } 2)\end{array}$ & $\begin{array}{l}\text { Esky } \\
(\mathrm{F})\end{array}$ \\
\hline & ---- & ---- & ----- & ------- & ----- & --- & - \\
\hline & -0.4 & 69.8 & 12.30 & Windward & 0.0 & -0.4 & \\
\hline Solar & 89.6 & 75.2 & 6.26 & Windward & 248.2 & 89.6 & \\
\hline
\end{tabular}

Frame Library Data

ID

Name

Source

U-value Edge GlzSys GlzSys Width

$+$

10 B-EX Alum Fra

Generic

Frame Edge Corr Width UC (PFD) Abs

Btu/h- Btu/h- inches Btu/h-

ft2-F ft2-F ft2-F

0.7850

$\mathrm{N} / \mathrm{A} \quad 5$

$\mathrm{N} / \mathrm{A}$

$\mathrm{N} / \mathrm{A}$

2.75

0.60

Divider Library Data

ID Name Source

U-value Edge GlzSys GlzSys Width

Div Edge Corr Width UC (PFD) Abs

Btu/h- Btu/h- inches Btu/h-

ft2-F ft2-F ft2-F

No Dividers for this Glazing system

Optical Properties for Glazing System '11 B-EX Single Pan'

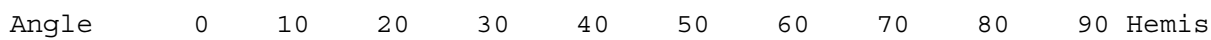

Vtc $: \begin{array}{lllllllllllll}0.898 & 0.898 & 0.897 & 0.895 & 0.887 & 0.868 & 0.820 & 0.703 & 0.439 & 0.000 & 0.820\end{array}$

Rf $\quad: \begin{array}{llllllllllllll}0.081 & 0.081 & 0.081 & 0.083 & 0.090 & 0.107 & 0.154 & 0.270 & 0.534 & 1.000 & 0.146\end{array}$

$\mathrm{Rb} \quad: \begin{array}{llllllllllll}0.081 & 0.081 & 0.081 & 0.083 & 0.090 & 0.107 & 0.154 & 0.270 & 0.534 & 1.000 & 0.146\end{array}$

Tsol : $\begin{array}{llllllllllllll}0.836 & 0.836 & 0.834 & 0.830 & 0.820 & 0.799 & 0.751 & 0.639 & 0.389 & 0.000 & 0.756\end{array}$

Rf $\quad: \begin{array}{lllllllllllll}0.075 & 0.075 & 0.075 & 0.077 & 0.082 & 0.099 & 0.143 & 0.252 & 0.505 & 1.000 & 0.136\end{array}$

$\mathrm{Rb} \quad: \begin{array}{llllllllllll}0.075 & 0.075 & 0.075 & 0.077 & 0.082 & 0.099 & 0.143 & 0.252 & 0.505 & 1.000 & 0.136\end{array}$

Abs1 : $0.0890 .0890 .090 \quad 0.093 \quad 0.097 \quad 0.101 \quad 0.105 \quad 0.108 \quad 0.106 \quad 0.000 \quad 0.098$

SHGCC: $\begin{array}{lllllllllllll}0.863 & 0.863 & 0.862 & 0.858 & 0.850 & 0.830 & 0.783 & 0.672 & 0.421 & 0.000 & 0.785\end{array}$

$\mathrm{TdW}-\mathrm{K}:-1.000$

Tdw-ISO: -1.000

Tuv $\quad:-1.000$

Window 5.2a v5.2.17a Report Page 3

$09 / 30 / 0908: 14: 35$

Temperature Distribution (degrees F)

Winter Summer

out In Out In

-.. - - -

$\begin{array}{lllll}\text { Lay1 } & 20.3 \quad 21.4 & 91.2 & 91.1\end{array}$ 


\section{E.2 Double-Pane Low-e Window With Wood Frame, Output From WINDOW 5}

The WINDOW 5 output listing below includes analysis results and inputs for the BESTEST-EX low-e window applied for cases L250EX and L300EX. The following inputs applied in the analysis are not listed:

- "NFRC" calculation mode is applied

- Environmental conditions are identical to "NFRC 100-2001", except:

$\circ$ Fixed combined interior surface coefficient $=1.070 \mathrm{Btu} /\left(\mathrm{h} \cdot \mathrm{ft}^{2} \cdot{ }^{\circ} \mathrm{F}\right)$

$\circ \quad$ Fixed combined exterior surface coefficient $=2.609 \mathrm{Btu} /\left(\mathrm{h} \cdot \mathrm{ft}^{2} \cdot{ }^{\circ} \mathrm{F}\right)$.

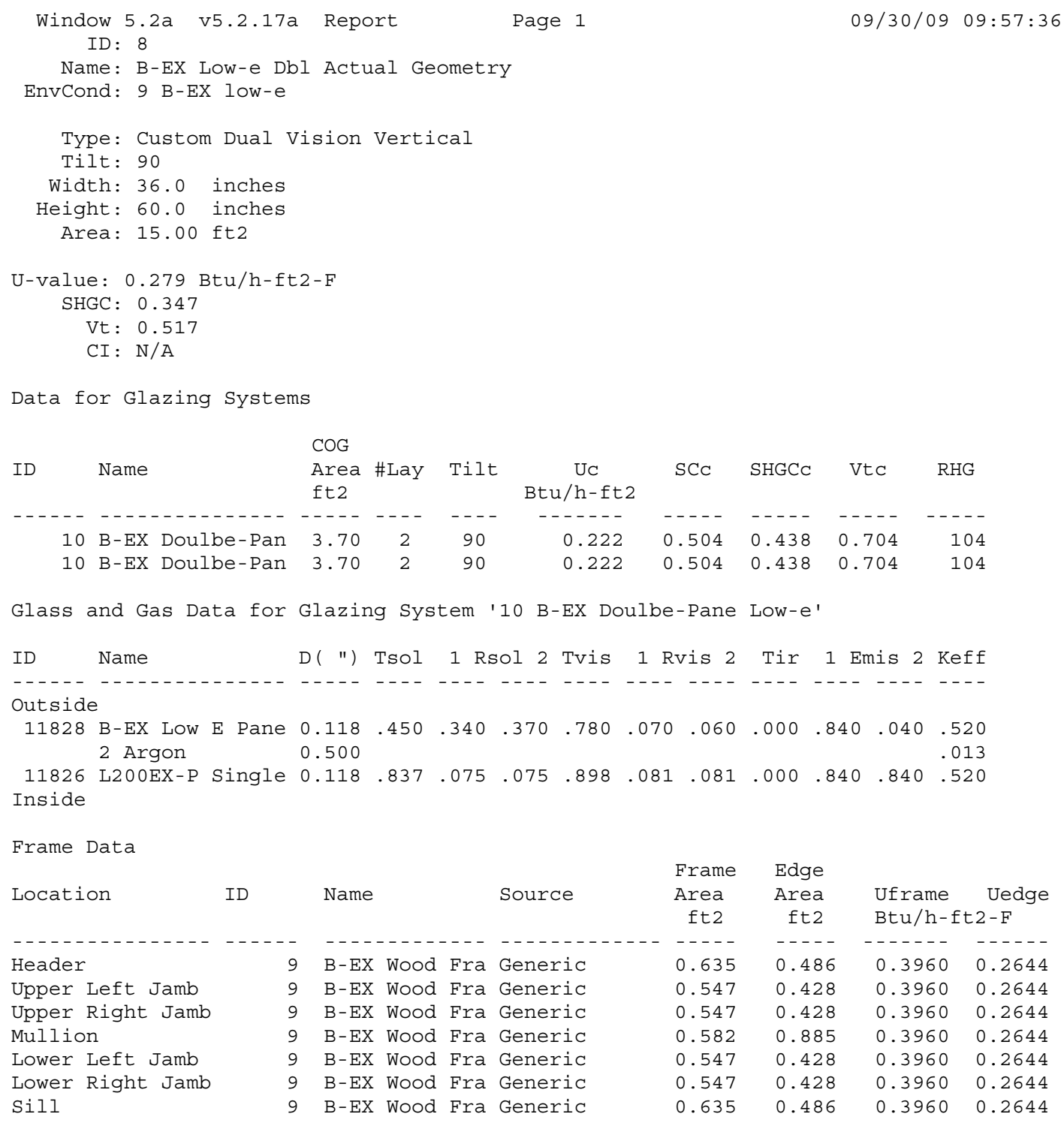




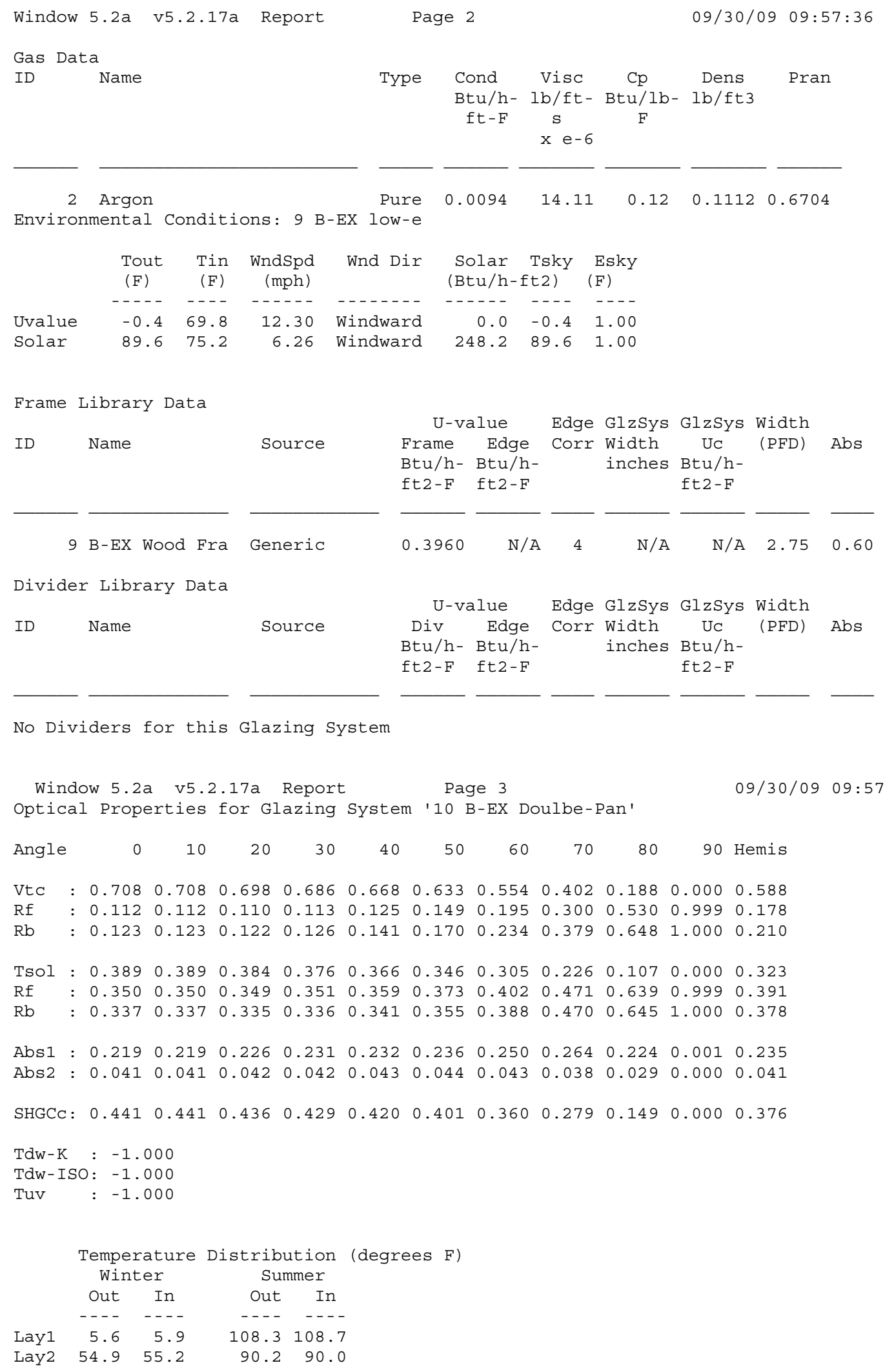

No Dividers for this Glazing System

Window 5.2a v5.2.17a Report Page 3 Optical Properties for Glazing System '10 B-EX Doulbe-Pan' 


\section{Appendix F Random Selection of Explicit Inputs for Case L200EX-C Reference Simulation Results}

Explicit inputs were randomly selected within the approximate input ranges (AIRs) assuming a triangular probability distribution. For this distribution the probability of selection is greatest at the nominal value and decreases linearly to zero at the minimum and maximum values. The triangular distribution may be either symmetric or asymmetric with respect to the nominal value; an asymmetric distribution is shown in Figure F-1.

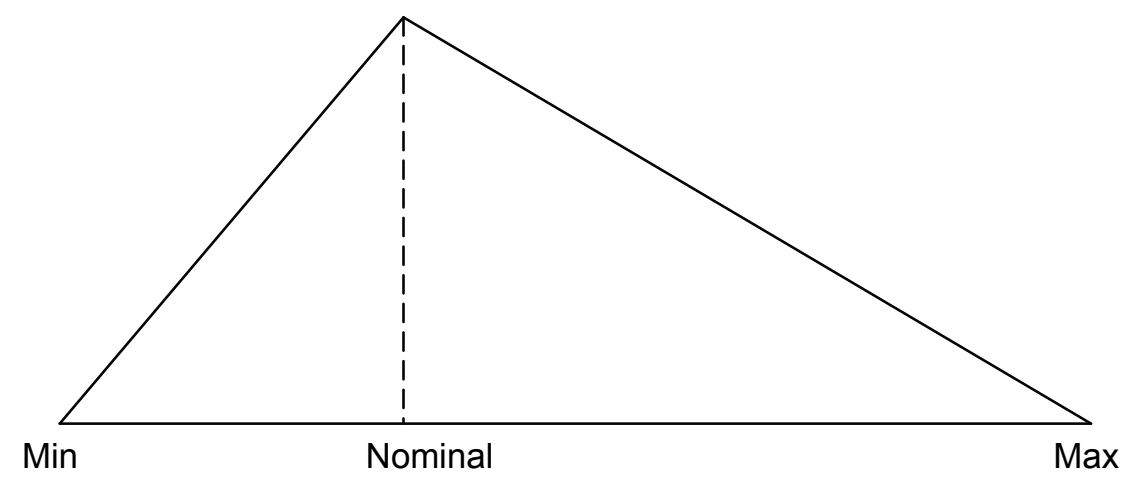

Figure F-1. Triangular probability distribution assumed for random generation of explicit inputs

Three types of explicit input sets were generated for the L200EX-C base-case scenarios: targeted high, targeted low, and fully random space-conditioning energy consumptions. Given approximate input ranges (min, max) for selected inputs (see Table F-1), sets of explicit values were generated using the following approaches:

1. Targeted High: Explicit inputs were selected randomly from the portion of the range (upper or lower) that led to increased space conditioning energy consumption versus nominal values. For inputs that have different effects in Las Vegas and Colorado Springs on the space conditioning loads (internal gains and solar absorptivity), the entire range was used.

2. Targeted Low: Explicit inputs were selected randomly from the portion of the approximate input range (upper or lower) that led to decreased space-conditioning energy consumption versus nominal values. For inputs that have different effects in Las Vegas and Colorado Springs on the space conditioning loads (internal gains and solar absorptivity), the entire range was used.

3. Fully Random: Explicit inputs were selected randomly from the entire range for each variable.

The Microsoft ExcelC 2003 "RAND" function was used to implement the triangular probability distribution and generate explicit input values within the AIRs listed in the test specification. According to Excel documentation, this pseudo-random number generator "returns an evenly distributed random number greater than or equal to 0 and less than 1." Supporting information is available at http://support.microsoft.com/kb/828795.

The "fully random" inputs were generated assuming a triangular probability distribution in Excel using the equation:

ExplicitValue $=\operatorname{IF}($ RAND\#<= $($ nom $-\min ) /(\max -\min ), \min +\operatorname{sqrt}(\operatorname{RAND\# }($ nom $-\min ) *(\max -\min ))$, $\max -\operatorname{sqrt}((1-\mathrm{RAND}) *(\max -\operatorname{nom}) *(\max -\min )))$ 
where "min," "nom," and "max" are the minimum, nominal, and maximum values of the approximate input range, respectively. The theoretical basis for this equation is provided in Kotz and van Dorp (2004). In Equation F-1, "RAND\#" refers to another cell where a random number is generated using the $=\mathrm{RAND}()$ function. The RAND() function should not be used within the equation above, because RAND\# must be the same value each time it is used. If RAND() were used in the equation above, three different random values would be generated and the equation would not correctly implement the probability distribution.

Equation F-1 was modified for the selection of "targeted" explicit inputs. For targeting the upper portion of the approximate input range (nominal value or greater), the value for "min" in Equation F-1 was set to "nom." For targeting the lower portion of the approximate input range (nominal value or less), the value of "max" in Equation F-1 was set to "nom." Table F-1 shows which portions of the approximate input ranges were used for the three different methods of selecting explicit inputs: targeted high ("High"), targeted low ("Low"), and fully random ("Random").

An Excel worksheet was created to generate sets of explicit inputs corresponding to targeted high, targeted low, and fully random cases. Each time the spreadsheet "recalculates," new random numbers are selected. The spreadsheet automatically calculates the appropriate values of input variables that depend on the explicit inputs. For example, if a random R-value of $16.3 \mathrm{~h} \cdot \mathrm{ft}^{2} \cdot{ }^{\circ} \mathrm{F} / \mathrm{Btu}$ is generated for the Ceiling/Attic/Roof R-value (see Table F-1), values for the attic fiberglass insulation thickness and joist thickness (see Table 1-6b) are calculated to match the selected Ceiling/Attic/Roof composite R-value. Explicit inputs were randomly selected for the parameters related to the variables listed in Table F-1 only. Even though inputs such as the attic fiberglass insulation and joist thickness have approximate input ranges in the test specification, the values of these inputs are not independent; they are uniquely determined once the values of the inputs in Table F-1 are generated. All explicit inputs and the calculated values for dependent inputs are not known by the participants testing software.

Sets of explicit inputs were created using a computer program that systematically recalculates the workbook (refreshes the randomly selected explicit inputs) and then outputs the explicit values to a text file. In the computer program the process is automatically repeated for the number of cases requested by the user. The output text file was used by another computer program that automatically creates input files for the EnergyPlus, SUNREL, and DOE2.1E simulations.

Using the methodology described above, 14 calibrated base-case scenarios were developed (developmental details follow):

- L200EX-C1H, targeted high space heating use

- L200EX-C2H, targeted low space heating use

- L200EX-C3H, fully random selection, near-nominal space heating use

- L200EX-C4H, fully random selection, high space heating use

- L200EX-C5H, fully random selection, low space heating use

- L200EX-C6H, fully random selection, mid-high space heating use

- L200EX-C7H, fully random selection, mid-low space heating use

- L200EX-C1C, targeted high space cooling use

- L200EX-C2C, targeted low space cooling use

- L200EX-C3C, fully random selection, near-nominal space cooling use

- L200EX-C4C, fully random selection, high space cooling use 
- L200EX-C5C, fully random selection, low space cooling use

- L200EX-C6C, fully random selection, mid-high space cooling use

- L200EX-C7C, fully random selection, mid-low space cooling use

Initially for both the heating and cooling cases, one targeted-high, one targeted-low, and 20 fully random sets of explicit inputs were generated (44 total sets). Each case was then simulated in EnergyPlus. The fully random cases were ranked according to annual space heating/cooling consumptions (sets of heating and cooling cases were considered separately). The fully random cases with space heating/cooling consumptions corresponding to the closest to nominal, highest, and lowest consumptions were initially selected for cases C3, C4 and C5 respectively (with a separate set of cases suffixed with " $\mathrm{H}$ " and "C" for heating and cooling, respectively).

For selecting the mid-high cases $\mathrm{C} 6 \mathrm{H}$ and $\mathrm{C} 6 \mathrm{C}$, and mid-low cases $\mathrm{C} 7 \mathrm{H}$ and $\mathrm{C} 7 \mathrm{C}$, mid-high and mid-low space heating/cooling consumption target values were calculated by averaging space heating/cooling consumptions according to:

$$
\begin{aligned}
& \text { "MidHigh" }=\frac{((L 200 E X P)+(C 4))}{2} \\
& \text { "MidLow" }=\frac{((L 200 E X P)+(C 5))}{2}
\end{aligned}
$$

where L200EXP is the nominal result from the building physics test base case (Case L200EX-P), and C4 and $\mathrm{C} 5$ are the highest and lowest results, as selected above (with a separate set of cases suffixed with " $\mathrm{H}$ " and "C" for heating and cooling, respectively).

\section{F.1 Non-HVAC (Base Load) Energy Use and Internal Gains}

Because base load energy use is often estimated from "swing" season or "off-" season utility data, uncertainty in base load energy use when a full year or more of utility billing data are available can be substantially less than the uncertainty range for sensible loads from electric and gas-fired appliances shown in Table F-1. Where monthly utility data are available, much of the uncertainty for internal gains may be attributable to the internal-gains-to-usage fractions, which is a relatively narrow band of uncertainty relative to the internal gains, and relative to some of the other parameters listed in Table F-1. 
Table F-1. Approximate Input Ranges (AIRs), Nominal Inputs, and Portions of AIRs Used for Generating Explicit Input Sets Corresponding to Low, Random, and High Space-Conditioning Energy Consumption

\begin{tabular}{|c|c|c|c|c|c|c|}
\hline \multirow[b]{2}{*}{ Input } & \multirow[b]{2}{*}{ Min } & \multirow[b]{2}{*}{ Max } & \multirow[b]{2}{*}{$\begin{array}{c}\text { L200EX } \\
\text { Nominal Value }\end{array}$} & \multicolumn{3}{|c|}{ Portion of AIR Used } \\
\hline & & & & $\begin{array}{c}\text { High } \\
\text { (C1) }\end{array}$ & $\begin{array}{l}\text { Low } \\
\text { (C2) }\end{array}$ & $\begin{array}{l}\text { Random } \\
\text { (C3-C7) }\end{array}$ \\
\hline $\begin{array}{l}\text { Ext. Wall R } \\
\left(\mathrm{h} \cdot \mathrm{ft}^{2} \cdot{ }^{\circ} \mathrm{F} / \mathrm{Btu}\right)\end{array}$ & 4.500 & 6.200 & 5.091 & Lower & Upper & Entire \\
\hline $\begin{array}{c}\text { Ceiling/Attic/Roof } \\
\mathrm{R}\left(\mathrm{h} \cdot \mathrm{ft}^{2} \cdot{ }^{\circ} \mathrm{F} / \mathrm{Btu}\right)\end{array}$ & 7.100 & 19.300 & 13.673 & Lower & Upper & Entire \\
\hline $\begin{array}{c}\text { Effective Leakage } \\
\text { Area @ 4Pa } \\
\text { (in. }{ }^{2} \text { ) }\end{array}$ & 137.4 & 215.9 & 196.3 & Upper & Lower & Entire \\
\hline $\begin{array}{l}\text { Sens Loads } \\
\text { Occupants } \\
\text { (Btu/day) }\end{array}$ & 4347 & 13041 & 8694 & Entire & Entire & Entire \\
\hline $\begin{array}{c}\text { Sens Loads } \\
\text { Elec } \\
\text { (Btu/day) }\end{array}$ & 18234 & 80000 & 36468 & Entire & Entire & Entire \\
\hline $\begin{array}{l}\% \text { Non-HVAC } \\
\text { Electricity to } \\
\text { Internal Gains }\end{array}$ & 60.0 & 90.0 & 75.0 & Entire & Entire & Entire \\
\hline $\begin{array}{c}\text { Sens Loads Gas } \\
\text { (Btu/day) }\end{array}$ & 7464 & 22392 & 14928 & Entire & Entire & Entire \\
\hline $\begin{array}{c}\text { \% Non-HVAC Gas } \\
\text { Energy to Internal } \\
\text { Gains }\end{array}$ & 20.0 & 35.0 & 27.5 & Entire & Entire & Entire \\
\hline Ext. Solar Abs. & 0.5 & 0.8 & 0.6 & Entire & Entire & Entire \\
\hline $\begin{array}{c}\text { Space } \\
\text { Conditioning } \\
\text { Season (\% of } \\
\text { annual load) }\end{array}$ & 90 & 99 & 95 & Upper & Lower & Entire \\
\hline $\begin{array}{l}\text { Heating Set Point } \\
\qquad\left({ }^{\circ} \mathrm{F}\right)\end{array}$ & 60.0 & 75.0 & 68.0 & Upper & Lower & Entire \\
\hline $\begin{array}{c}\text { Furnace Efficiency } \\
(\%)\end{array}$ & 60.0 & 80.0 & 70.0 & Lower & Upper & Entire \\
\hline $\begin{array}{c}\text { Cooling Set Point } \\
\left({ }^{\circ} \mathrm{F}\right)\end{array}$ & 71.0 & 86.0 & 78.0 & Lower & Upper & Entire \\
\hline Cooling COP & 2.5 & 3.5 & 3.0 & Lower & Upper & Entire \\
\hline
\end{tabular}

Note: All explicit inputs are selected independently for each space heating and space cooling base-case scenario, except heating set point and furnace efficiency are selected for space heating cases only, and cooling set point and cooling COP are selected for space cooling cases only. 


\section{Appendix G Example Results}

This appendix presents:

- Building physics test cases reference results (see Section G.1)

- Benefit of calibration discussion (see Section G.2)

- Improvements to tested software and importance of simulation trials (see Section G.3).

\section{G.1 Building Physics Test Cases Reference Results}

Reference results were developed using:

- DOE-2.1E Version JJHirsch PC 2.1En136 (DOE-2 Reference Manual 1981, DOE-2 Supplement 1994)

- EnergyPlus Version 3.1 (EnergyPlus Input Output Reference 2009)

- $\quad$ SUNREL Version 1.14 (Deru et al. 2002)

Figure G-1 and Table G-1 show the building physics ("“-P") cases reference results for the heating cases. Figure G-2 and Table G-2 show the "-P" reference results for the cooling cases. An electronic version of the results is provided with $B$-EX-Phase-1-Ref-P-Results.xls included with the accompanying electronic files. Cell addresses for finding this data within the .xls file are given in small font below the tables.

Only the results for the "-P" test cases are shown in the figures and the tables. For the calibrated energy savings ("-C") test cases, reference simulation results and randomly selected explicit inputs used in the reference simulations are intentionally not given for blind testing. 
Annual Gas Usage or Savings

Buildings Physics Heating Tests

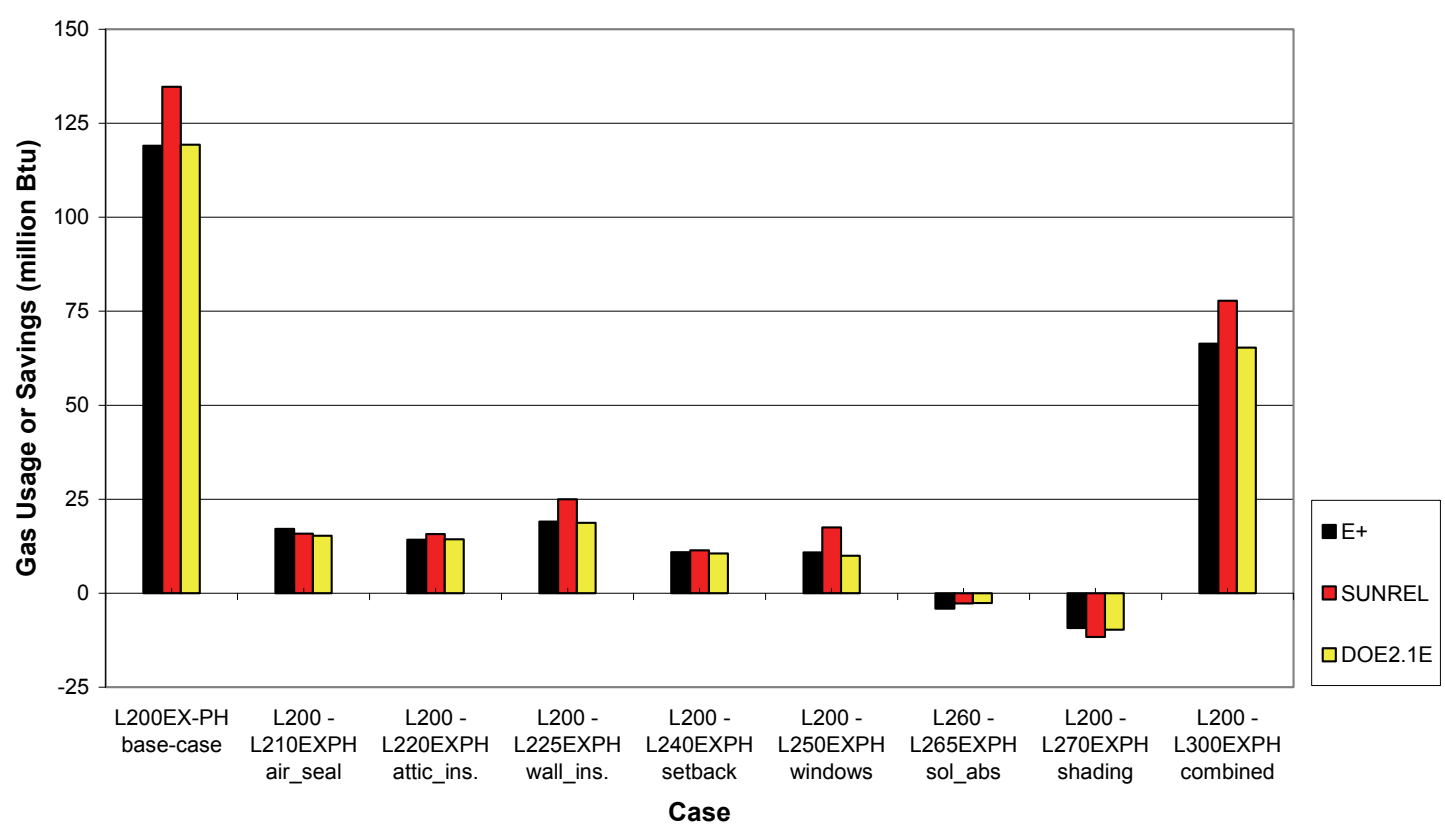

Figure G-1. Building physics heating tests: Reference simulation results

Annual Electricity Usage or Savings

Buildings Physics Cooling Tests

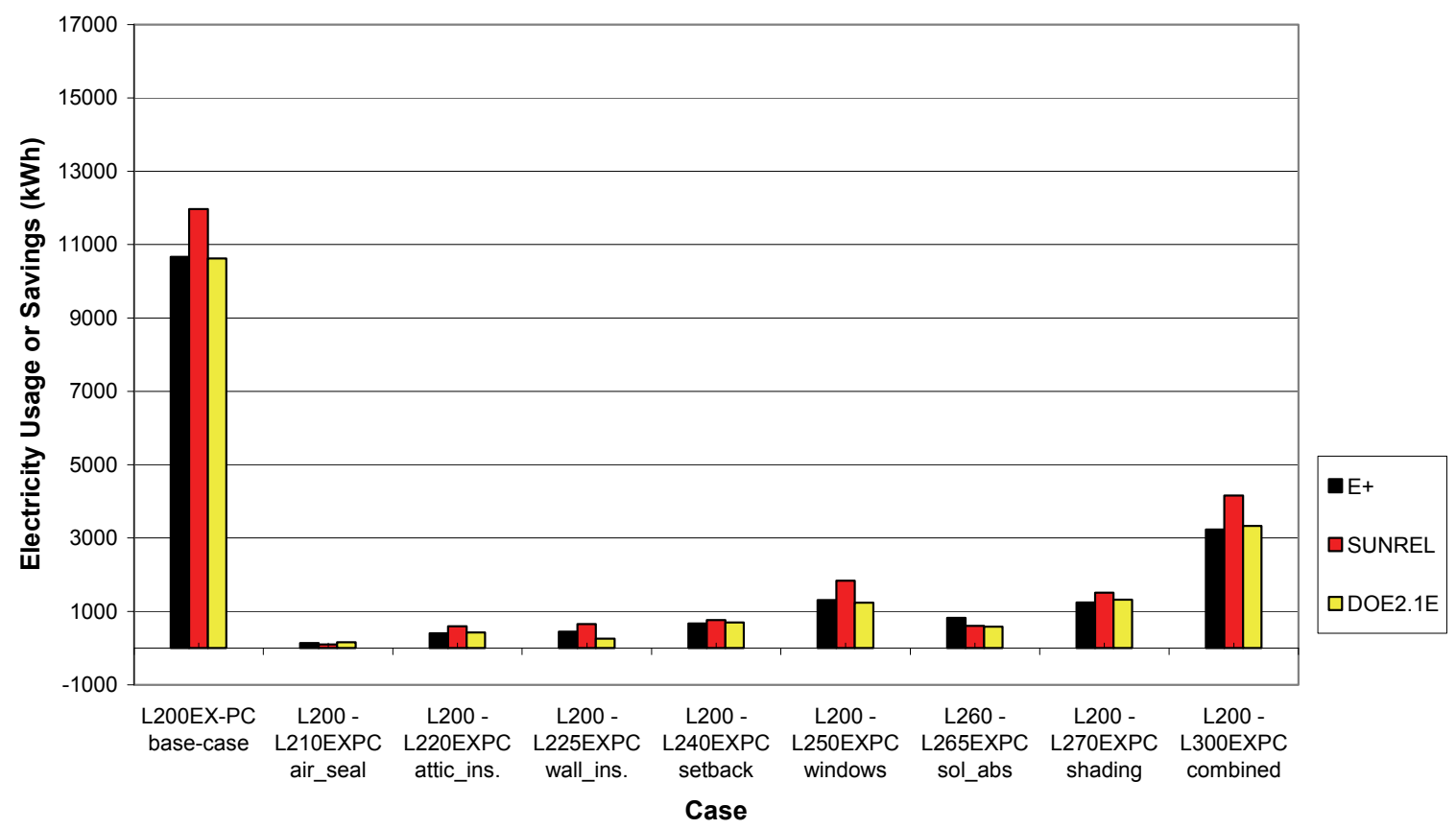

Figure G-2. Building physics cooling tests: Reference simulation results 
Table G-1. BESTEST-EX Building Physics Heating Tests Reference Results

\begin{tabular}{||l|rrr||}
\hline \multicolumn{4}{||l}{ Total Annual Gas Consumption and Savings (million Btu/y) } \\
Case & EnergyPlus & SUNREL & DOE2.1E \\
\hline L200EX-PH base-case & 119.01 & 134.68 & 119.32 \\
\hline L200 - L210EXPH air_seal & 17.14 & 15.88 & 15.33 \\
L200 - L220EXPH attic_ins. & 14.27 & 15.74 & 14.34 \\
L200 - L225EXPH wall_ins. & 19.10 & 25.00 & 18.69 \\
L200 - L240EXPH setback & 10.91 & 11.42 & 10.56 \\
L200 - L250EXPH windows & 10.86 & 17.50 & 9.92 \\
L260 - L265EXPH sol_abs & -4.08 & -2.74 & -2.58 \\
L200 - L270EXPH shading & -9.27 & -11.66 & -9.65 \\
L200 - L300EXPH combined & 66.38 & 77.81 & 65.34 \\
\hline \hline
\end{tabular}

B-EX-Phase-1-Ref-P-Results.XLS: GasData! A256:F269

3-May-2010

Table G-2. BESTEST-EX Building Physics Cooling Tests Reference Results

\begin{tabular}{||l|rrr||}
\hline \multicolumn{4}{|l||}{ Total Annual Electricity Consumption and Savings (kWh/y) } \\
Case & EnergyPlus & SUNREL & DOE2.1E \\
\hline L200EX-PC base-case & 10664 & 11966 & 10622 \\
\hline L200 - L210EXPC air_seal & 140 & 103 & 156 \\
L200 - L220EXPC attic_ins. & 405 & 596 & 428 \\
L200 - L225EXPC wall_ins. & 454 & 656 & 259 \\
L200 - L240EXPC setback & 671 & 765 & 700 \\
L200 - L250EXPC windows & 1310 & 1840 & 1234 \\
L260 - L265EXPC sol_abs & 821 & 609 & 586 \\
L200 - L270EXPC shading & 1247 & 1508 & 1325 \\
L200 - L300EXPC combined & 3235 & 4161 & 3330 \\
\hline \hline
\end{tabular}

B-EX-Phase-1-Ref-P-Results.XLS: ELECclgData! A267:E280

3-May-2010 


\section{G.2 Benefit of Calibration}

One goal of the BESTEST-EX process is to estimate the benefit of utility bill calibration. In this section the BESTEST-EX Working Group participant results from a preliminary field trial are analyzed to provide an estimate of this benefit. The general approach is to compare the errors of energy savings predictions using calibrated models versus the errors of predictions using uncalibrated models. Results analyzed in this section are not final BESTEST-EX results. Rather, they represent a snapshot in time during a development process where modifications and improvements were being made to the test specification, reference simulation modeling, and participant software tools.

"Nominal" values from the test specification tables (see Section 1) are used for inputs in the physics test cases. These nominal values are analogous to the reported values by an auditor or the default values in the software. Because nominal inputs are used for the physics retrofit cases, the physics test results can be thought of as "uncalibrated" energy savings predictions.

The nominal values reported by an auditor have uncertainty. For example, the nominal ELA for a residential building may be reported as 196 in. ${ }^{2}$, but because of measurement uncertainty, variations in weather conditions, modeling assumptions, etc., the value is better-represented using an uncertainty range: e.g., ELA = $196 \pm 20$ in. $^{2}$. The "true" or "as-installed" value for the input is likely to fall within the input range.

Audit software providers often calibrate or "true-up" the pre-retrofit base case model to utility bills by varying inputs away from the nominal, reported, or default values. For the utility bill calibration cases in BESTEST-EX, users are given nominal inputs, uncertainty ranges, and pre-retrofit billing data (generated by the reference programs using randomly selected explicit or "as-installed" inputs). The tested software calibrates the base case model to the utility bills by varying key model inputs away from the nominal, reported, or default values and then predicts energy savings for the different retrofit measures using the calibrated model. Because software tools calibrate to utility bills by varying the key model inputs, the calibration test results can be thought of as "calibrated" energy savings predictions.

The analysis that follows is based on the results of the last full simulation trial of test cases with the BESTEST-EX Working Group participants before the publication of this test procedure. The draft of the test specification used in the simulation trial has the following important differences from the final version of the test procedure:

1. The randomly selected reference simulation inputs for the calibration cases in the simulation trial are different from those selected for the calibration cases in the final version of the test procedure.

2. External shading was not modeled in the combined physics cooling retrofit case (L300EX-PC) for the simulation trial.

For the simulation trial, six results sets were submitted from five different software providers. All six results sets were complete for the heating test cases. However, one set was not complete for the cooling test cases and therefore was eliminated from all cooling analysis. Another program did not model a particular retrofit measure for the combined cooling cases and therefore was eliminated from analysis for that subset of cases (L300EX-PC and L300EX-CC).

\section{G.2.1 Analysis Approach}

The benefit of calibration analysis was performed under the following assumptions:

1. Because the utility bills in BESTEST-EX are the average of the reference program simulations using the same set of randomly selected inputs for a given simulation trial, Working Group participant energy savings predictions are compared to average energy savings predictions of the reference programs. 
2. Working Group participant results for the physics cases are applied as uncalibrated energy savings predictions.

3. Working Group participant results for the utility bill calibration cases are calibrated energy savings predictions.

To compare results from physics test cases and calibration test cases, the definition of the retrofit measures must be equivalent for both types of test cases. For example, in the physics wall insulation retrofit (L225EX-P), 3.5 in. of cellulose is blown into the wall cavity. Although the overall wall R-value for the wall insulation calibration cases (L225EX-C) can differ from the physics case, the retrofit measure is identical; $3.5 \mathrm{in}$. of cellulose is blown into the wall cavity. The same definition of the retrofit measure allows for a meaningful comparison between the physics (uncalibrated) and utility bill calibration (calibrated) results. In some instances there are differences between the retrofit specifications for the physics and calibration cases; these are:

1. For the physics air-sealing retrofit (L210EX-P) the ELA is reduced from $196.3 \mathrm{in}^{2}$ to $98.1 \mathrm{in} .^{2}$, corresponding to a decrease in ELA of $98.2 \mathrm{in}^{2}$. The air-sealing retrofit for the calibration cases specifies a 100 in. $^{2}$ decrease in ELA from the randomly selected value. For the purpose of this analysis, the 1.8 in. $^{2}$ difference in ELA reduction between the physics and calibration test cases is neglected.

2. External shading (L270-EX) is not included in the calibration cases and therefore is not considered in this analysis.

3. The cool roof retrofit for the physics test cases is defined as a decrease in the roof solar absorptance from 0.8 to 0.2 (L260EX-P-L265EX-P). In the calibration cases it is defined as absolute change from the randomly selected value (between 0.5 and 0.8 ) to 0.2 (L200EX-C $-\mathrm{L} 265 \mathrm{EX}-\mathrm{C})$. Because this measure is defined differently for the physics and calibration test cases, the individual cool roof retrofit case (L265EX) is excluded from this analysis. The cool roof retrofit is not excluded, however, from the combined retrofit cases (L300EX) because the definition of the retrofit measure is similar between the physics and calibration cases (the preretrofit value for the roof solar absorptance is the nominal value of 0.6 ).

\section{G.2.2 Graphical Comparison}

In this section results from the BESTEST-EX Working Group field trial are presented in graphical form. The benefit of calibration is examined for the following calibration scenarios:

- $\quad \mathrm{C} 3=$ Fully random explicit input selection, near nominal space heating/cooling consumption

- $\quad \mathrm{C} 4=$ Fully random explicit input selection, high space heating/cooling consumption

- $\quad \mathrm{C} 5=$ Fully random explicit input selection, low space heating/cooling consumption

- $\quad$ C6 = Fully random explicit input selection, mid-high space heating/cooling consumption

- $\quad \mathrm{C} 7=$ Fully random explicit input selection, mid-low space heating/cooling consumption

The targeted cases ( $\mathrm{C} 1$ and $\mathrm{C} 2)$ are not analyzed because the uncertainty of the key model inputs is artificially reduced by selecting explicit inputs from portions of the ranges that lead to high or low space heating/cooling consumption. The calibration scenarios are described in detail in Section 1.3.1.2 and Appendix F.

Figures G-3 through G-7 and Figures G-8 through G-12 show the benefit of calibration for the heating and cooling calibration scenarios, respectively. The following abbreviations apply to the figures: 
$=$ Average of reference program energy savings predictions using randomly selected inputs [million Btu or $\mathrm{kWh}$ ]

WG CAL = Average Working Group calibrated energy savings prediction [million Btu or kWh]

WG UNCAL = Average Working Group uncalibrated energy savings prediction [million Btu or $\mathrm{kWh}]$

For the sets of individual results used to develop the average values shown, the error bars represent one standard deviation above those values. For format clarity, the error bar extensions for one standard deviation below the average values are not shown.

C3H (Fully Random, Near Nominal)

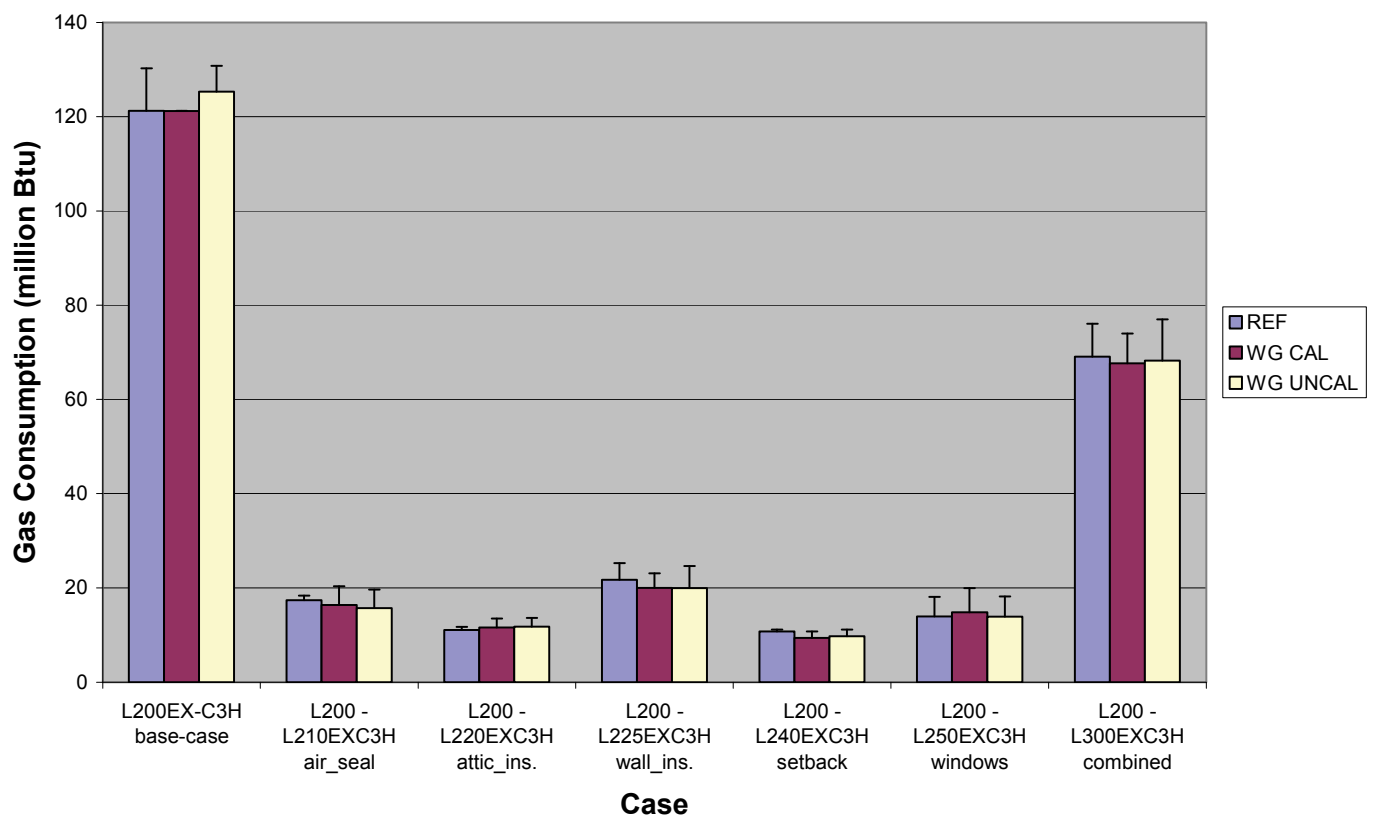

Figure G-3. Field trial results for fully random, near nominal heating scenario $(\mathrm{C} 3 \mathrm{H})$ 
C4H (Fully Random, High)

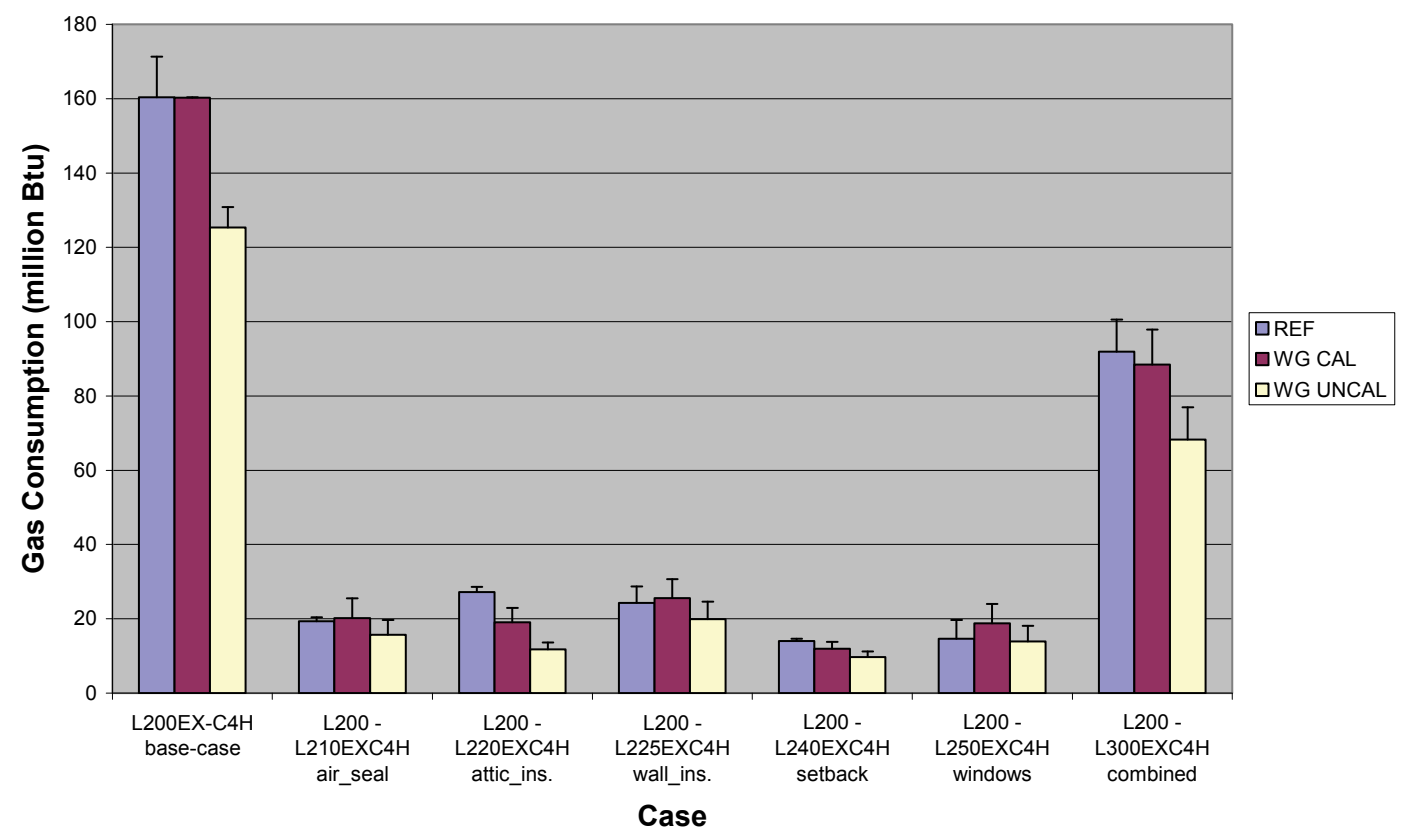

Figure G-4. Field trial results for fully random, high heating scenario (C4H)

C5H (Fully Random, Low)

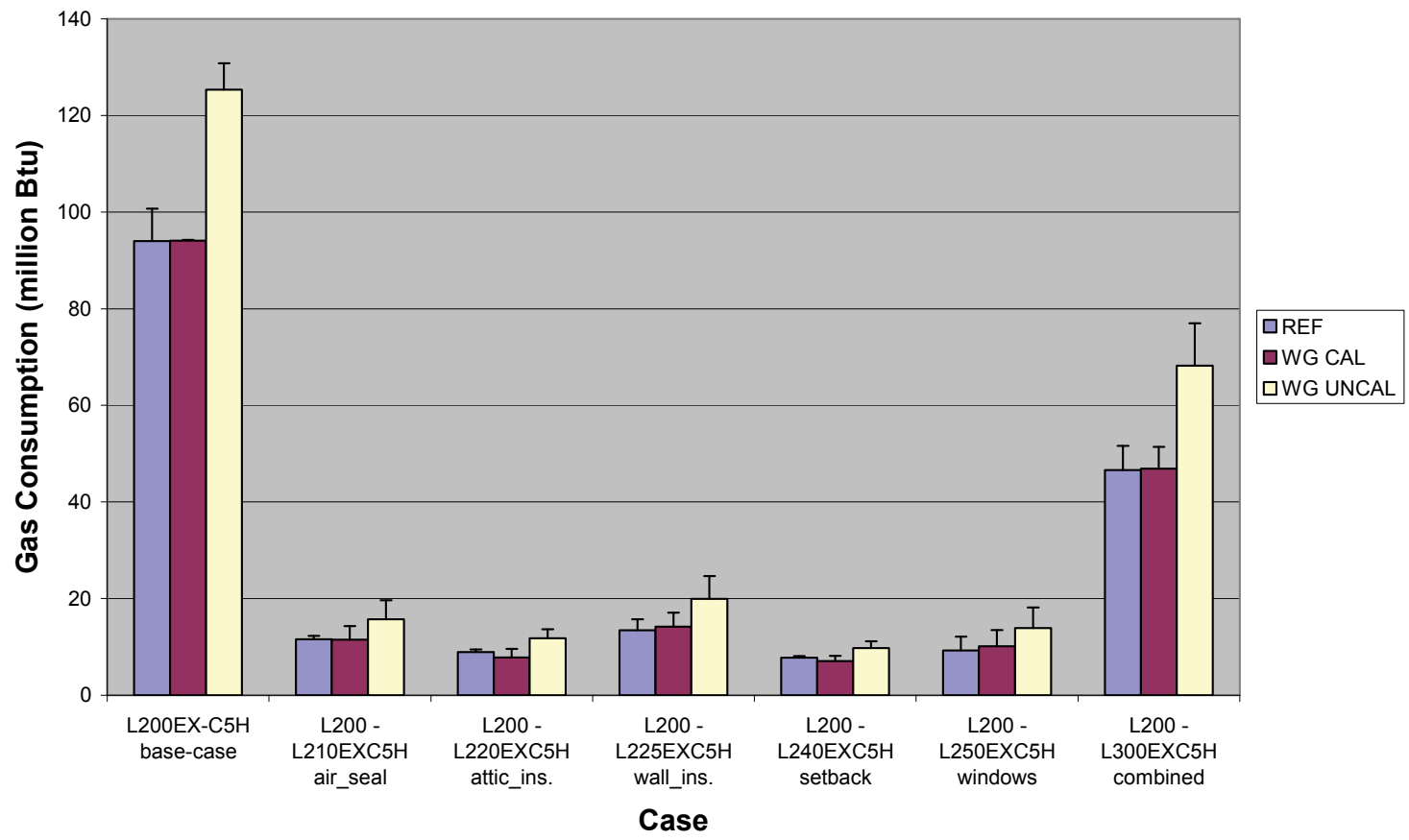

Figure G-5. Field trial results for fully random, low heating scenario (C5H) 
C6H (Fully Random, Mid-High)

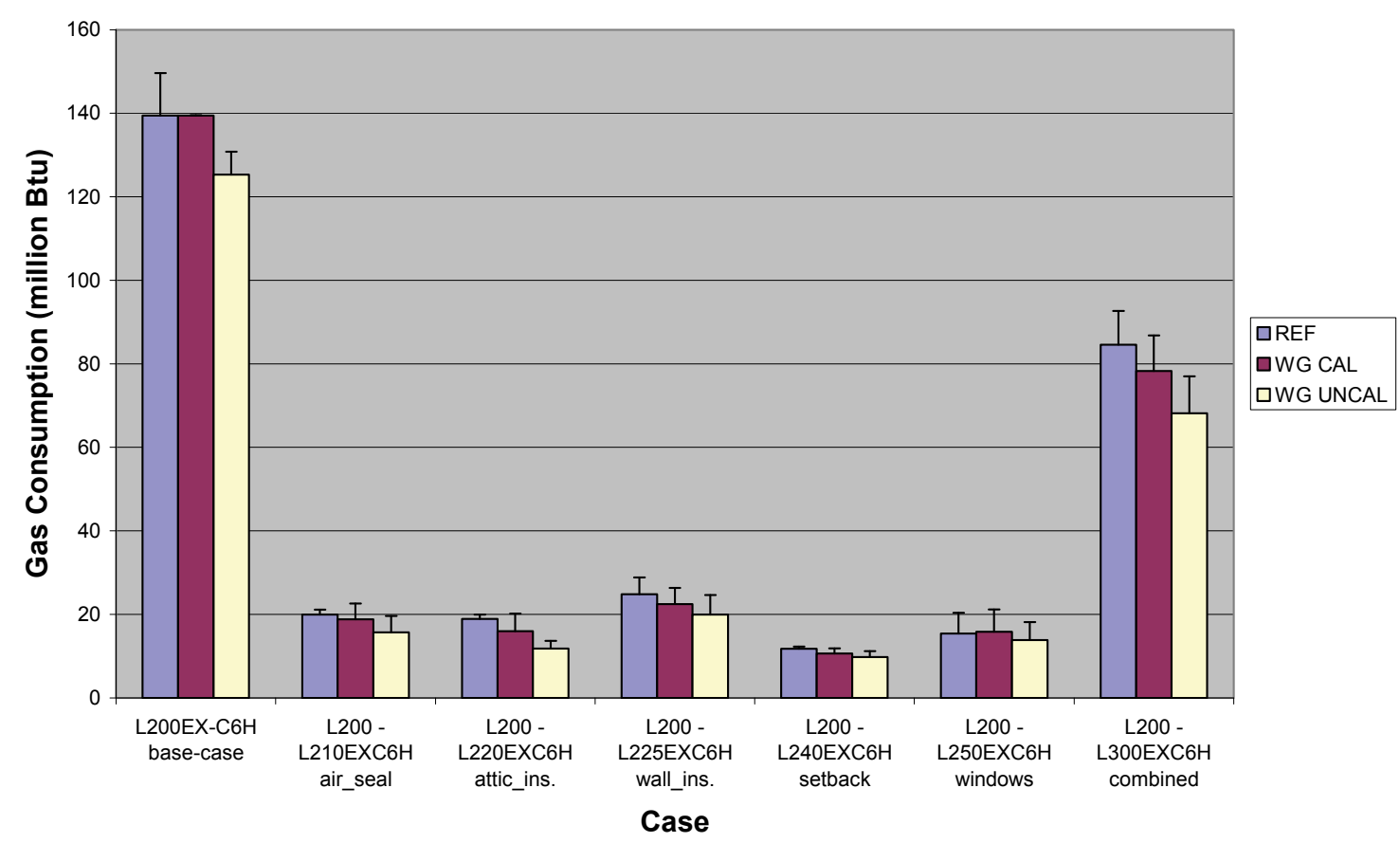

Figure G-6. Field trial results for fully random, mid-high heating scenario (C6H)

C7H (Fully Random, Mid-Low)

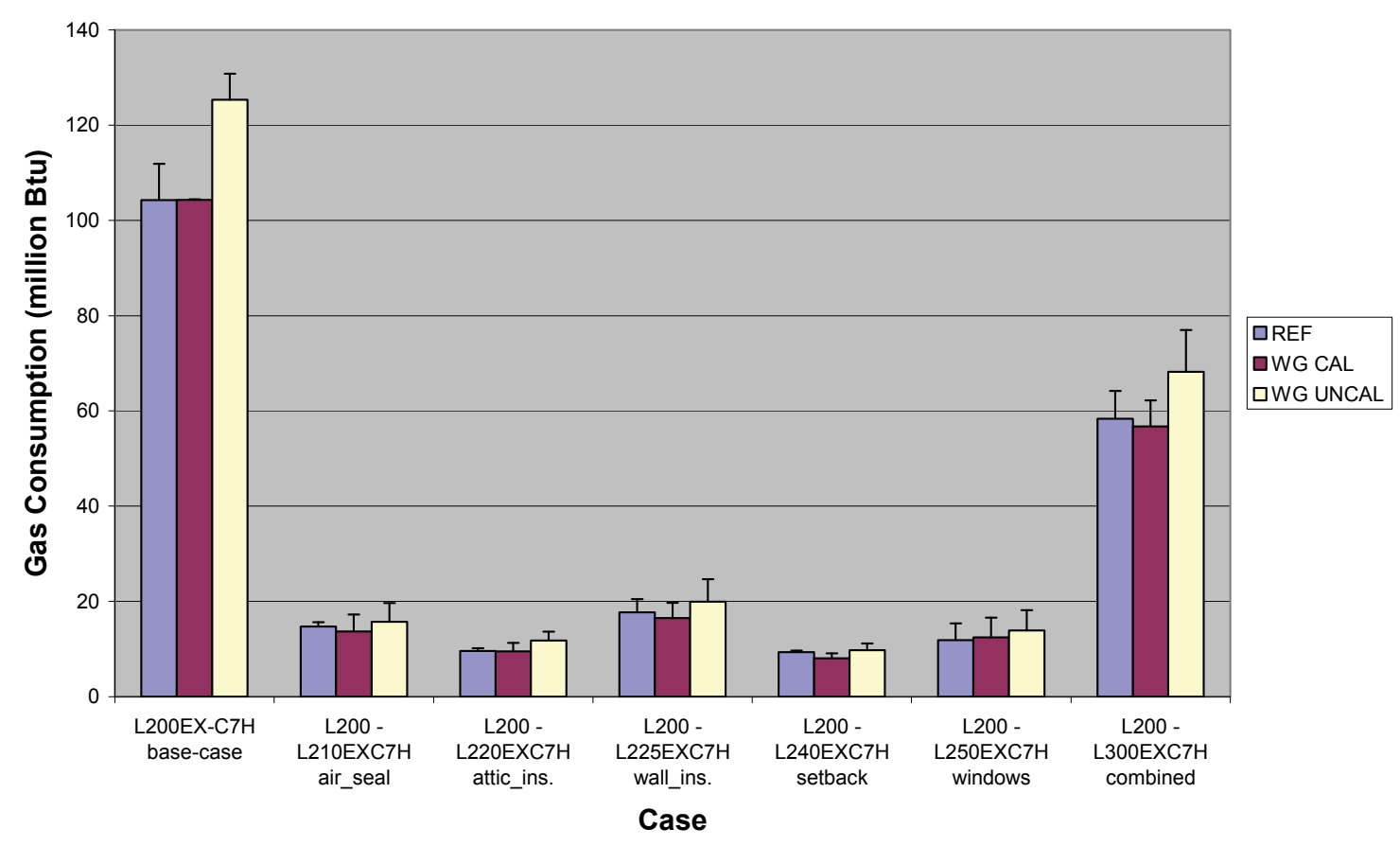

Figure G-7. Field trial results for fully random, mid-low heating scenario (C7H) 


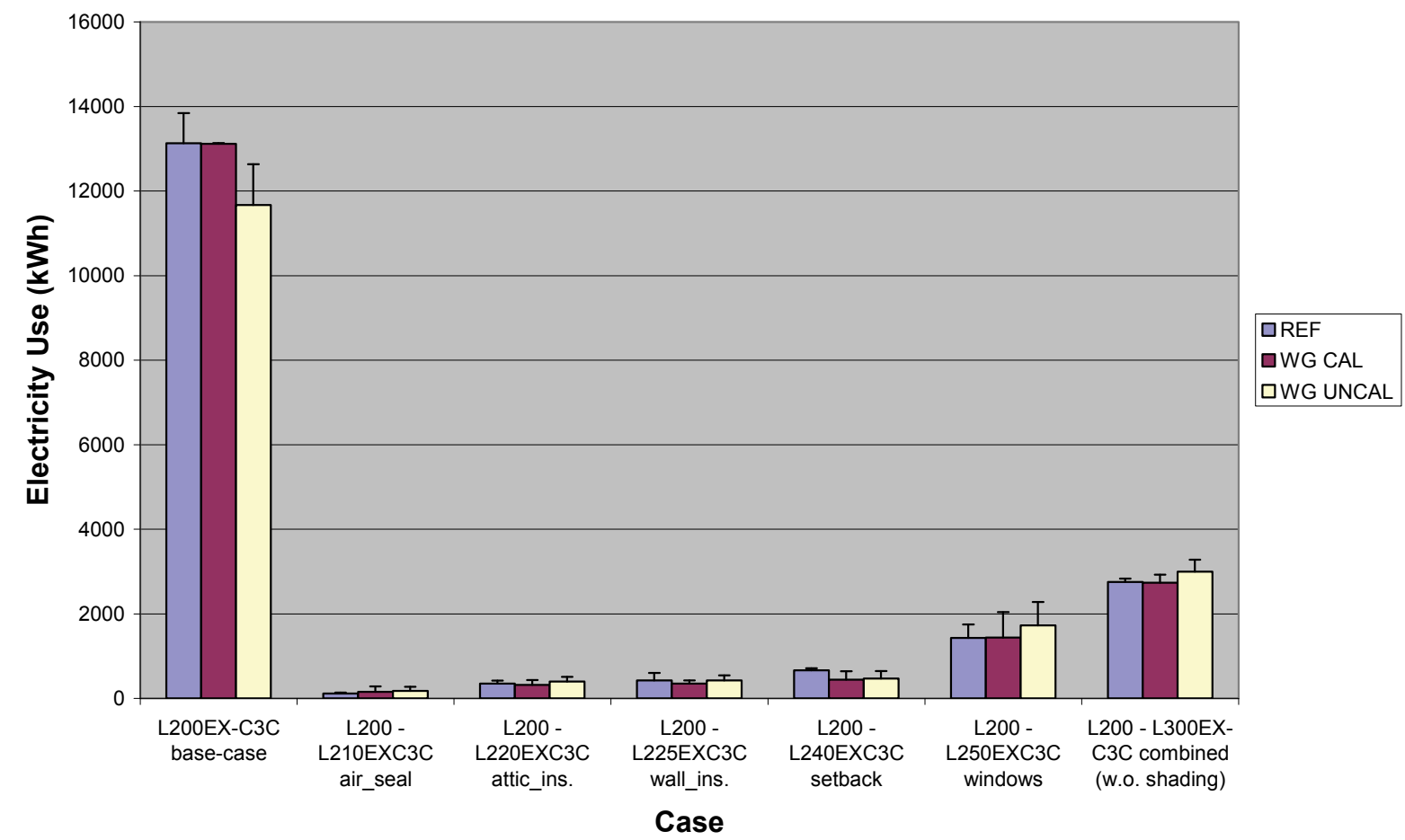

Figure G-8. Field trial results for fully random, near nominal cooling scenario (C3C) C4C (Fully Random, High)

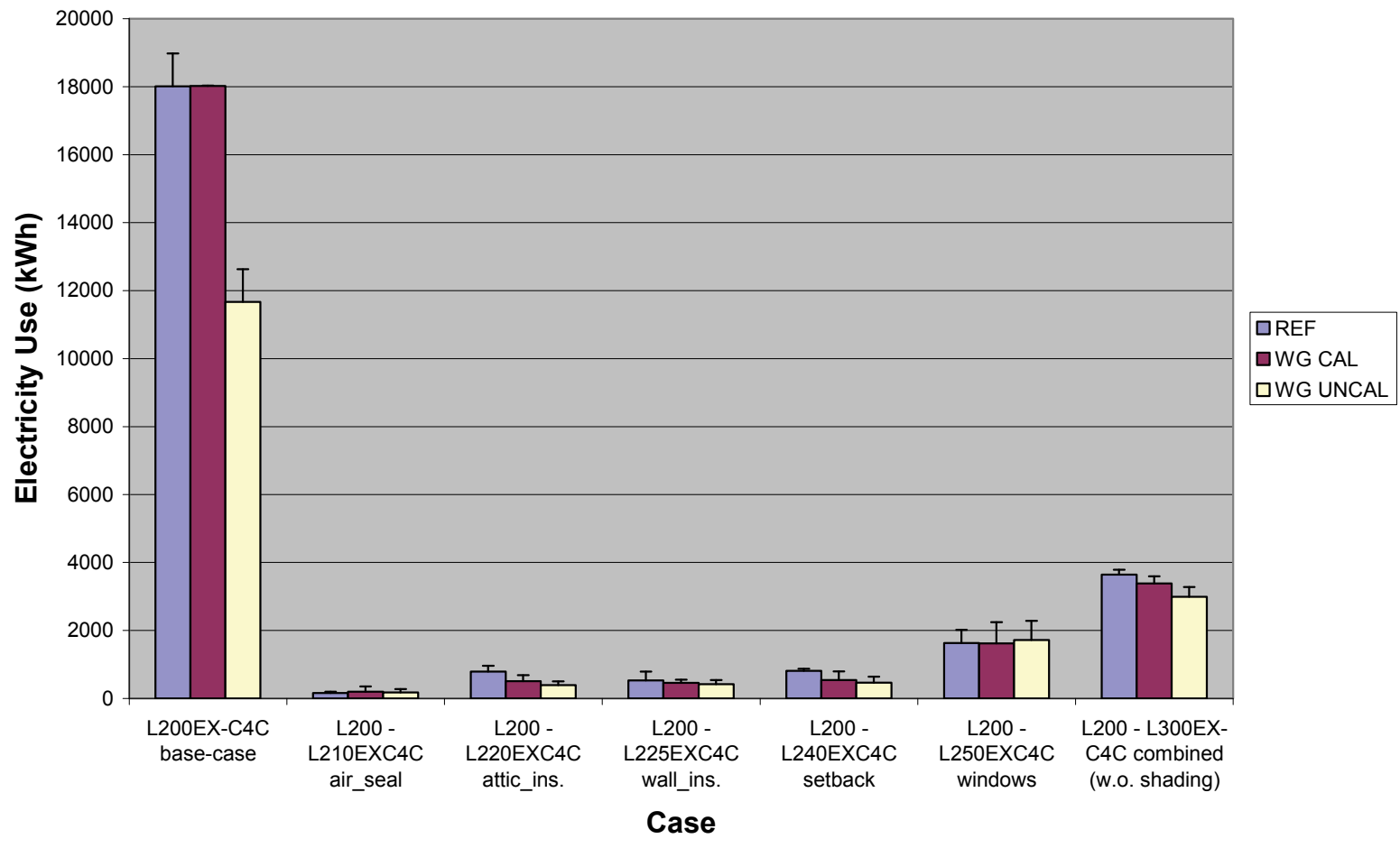

Figure G-9. Field trial results for fully random, high cooling scenario (C4C) 


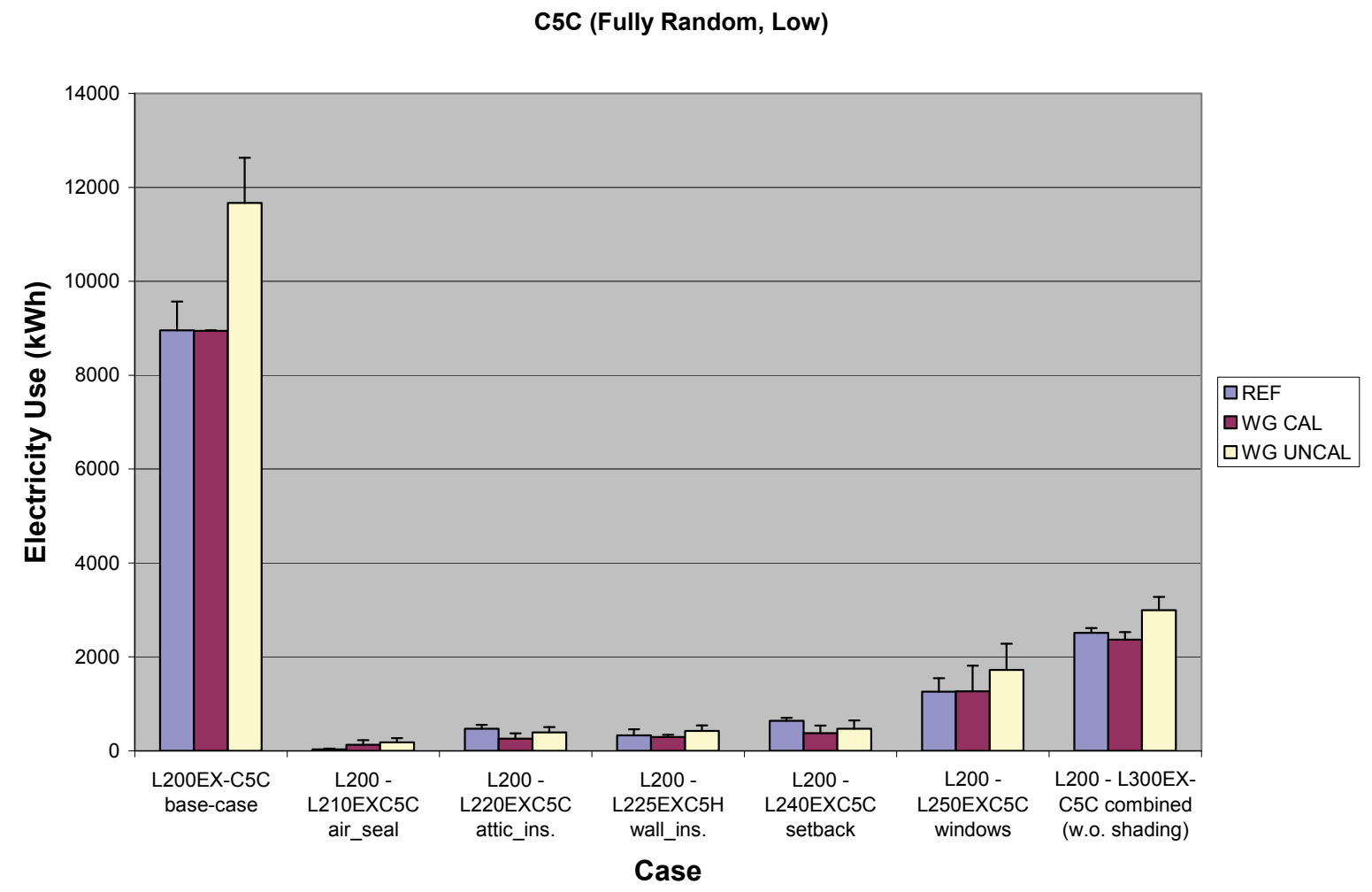

Figure G-10. Field trial results for fully random, low cooling scenario (C5C)

C6C (Fully Random, Mid-High)

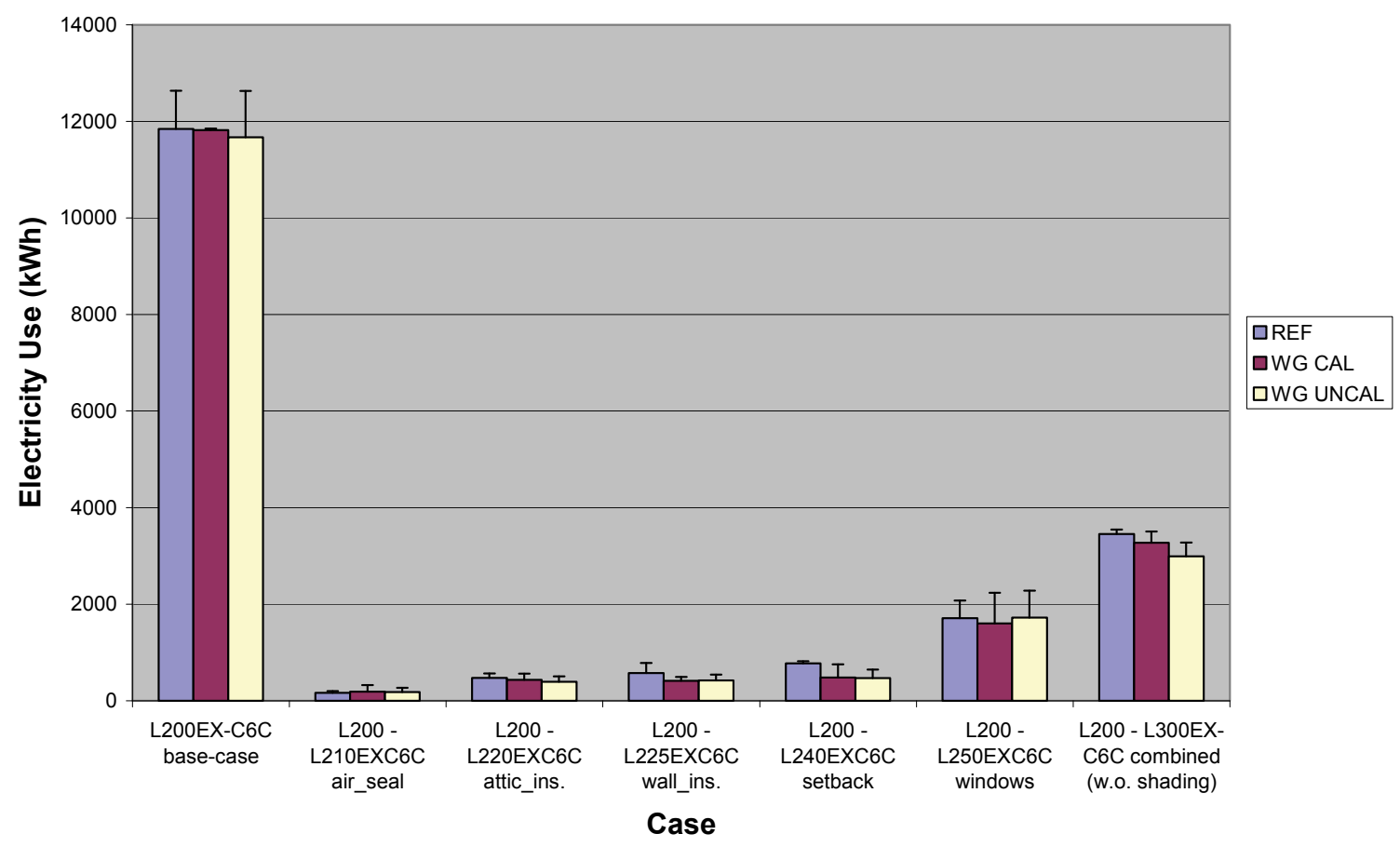

Figure G-11. Field trial results for fully random, mid-high cooling scenario (C6C) 
C7C (Fully Random, Mid-Low)

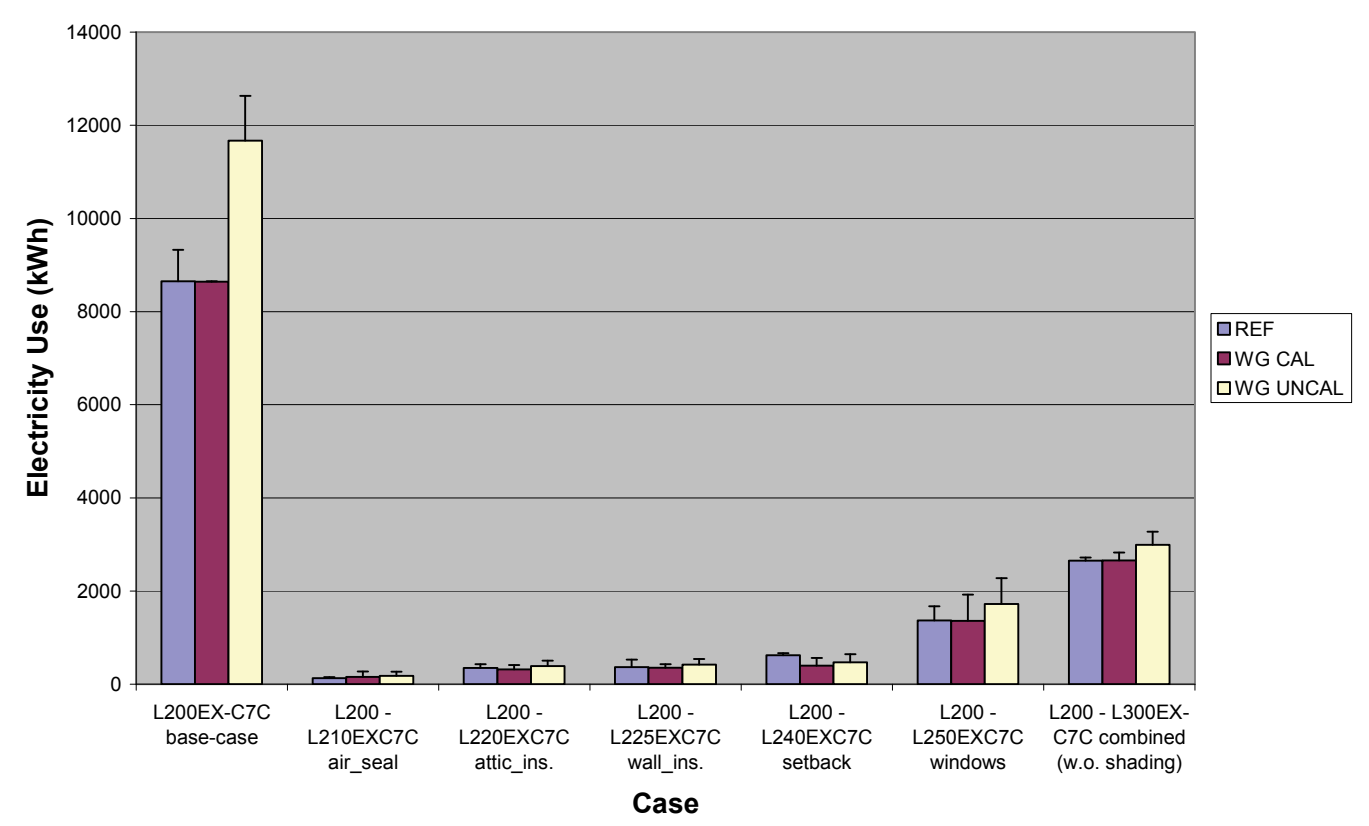

Figure G-12. Field trial results for fully random, mid-low cooling scenario (C7C)

\section{G.2.3 Quantitative Comparison}

Figures G-3 through G-12 are graphical representations of the benefit of calibration. Qualitatively, they show that calibration generally improves the accuracy of the average Working Group participant energy savings predictions relative to the average reference program energy savings predictions. The improvement tends to increase as the difference between uncalibrated energy consumption and reference utility bill consumption increases (i.e., as the degree of calibration increases).

The benefit of calibration $(B o C)$ can be quantified. For example, the mean absolute error for the calibrated and uncalibrated energy savings predictions can be calculated using the equations

$$
\begin{aligned}
& \text { ERRcal } \left.=\frac{1}{N} \sum_{i=1}^{N}\left|W G C A L_{i}-R E F\right| \quad \text { [million Btu or } \mathrm{kWh}\right] \\
& \text { ERRuncal }=\frac{1}{N} \sum_{i=1}^{N}\left|W G U N C A L_{i}-R E F\right| \quad \text { [million Btu or kWh] }
\end{aligned}
$$

Where:

$\begin{array}{lll}N & = & \begin{array}{l}\text { Number of Working Group participant results sets } \\ \text { Calibrated energy savings prediction of individual Working Group } \\ \text { program }\end{array} \\ W G C A L_{i} & = & \begin{array}{l}\text { Uncalibrated energy savings prediction of individual Working Group } \\ \text { program }\end{array} \\ R E F & = & \text { Average reference program energy savings prediction }\end{array}$

The mean absolute error does not distinguish between underprediction and overprediction because the absolute value is taken for each residual. 
The $B o C$ can then be defined as the decrease in mean absolute error of participant energy savings predictions:

$$
\text { BoC }=\text { ERRuncal }- \text { ERRcal } \quad[\text { million Btu or } \mathrm{kWh}]
$$

When $B o C$ is positive, energy savings predictions are improved by calibration. $B o C$ has the same units as the consumption [million Btu or $\mathrm{kWh}$ ] and therefore can be compared to overall energy savings and converted to monetary values based on fuel prices. This is one of many approaches that can be used to quantify the benefit of calibration. For example, the root mean square error, mean of the residuals, standard deviation of the residuals, etc. could be examined in future analysis.

Table G-3 indicates the sign of $B o C$ ("Y" when $B o C$ is positive, "N" when $B o C$ is not positive) for the different fully random scenarios and retrofit measures in the Working Group field trial.

Table G-3. Benefit of Calibration (BoC) for Working Group Field Trial

\begin{tabular}{|c|c|c|c|c|c|c|c|c|c|}
\hline IS BoC >0? & C3 $\mathrm{C}$ & $\begin{array}{l}\text { eati } \\
\text { C55 }\end{array}$ & & & & $\begin{array}{r}c \\
3 \mathrm{C}\end{array}$ & $\begin{array}{l}0 \\
0\end{array}$ & $5 \mathrm{Ce}$ & $\mathrm{C}$ \\
\hline Air Seal & $\mathrm{YN}$ & $\mathrm{Y}$ & $\mathrm{Y}$ & $\mathrm{Y}$ & $\overline{\mathrm{N}}$ & $\mathrm{N}$ & $\mathrm{Y}$ & $\bar{N}$ & $\mathrm{~N}$ \\
\hline Attic Insulation & $Y \quad Y$ & $\mathrm{Y}$ & $\mathrm{Y}$ & $\mathrm{Y}$ & $\mathrm{N}$ & $\mathrm{Y}$ & $\mathrm{N}$ & $\mathrm{N}$ & $\mathrm{Y}$ \\
\hline Wall Insulation & $\mathrm{Y} Y$ & $\mathrm{Y}$ & $\mathrm{Y}$ & $\mathrm{Y}$ & Y & $Y$ & Y & $\mathrm{N}$ & $Y$ \\
\hline Programmable Thermostat & $\mathrm{N} \mathrm{Y}$ & $\mathrm{Y}$ & $\mathrm{Y}$ & $\mathrm{N}$ & $\mathrm{N}$ & $\mathrm{Y}$ & $\mathrm{N}$ & $\mathrm{N}$ & $\mathrm{N}$ \\
\hline Low-e Windows & $\mathrm{N} \mathrm{N}$ & $\mathrm{Y}$ & $\mathrm{N}$ & $\mathrm{Y}$ & Y & Y & $\mathrm{Y}$ & $\mathrm{Y}$ & $\mathrm{Y}$ \\
\hline Combined Retrofit & $\mathbf{Y} \mathbf{Y}$ & $\mathbf{Y}$ & $\mathbf{Y}$ & $\mathbf{Y}$ & $\mathbf{Y}$ & $\mathbf{Y}$ & $\mathbf{Y}$ & $\mathbf{Y}$ & $\mathbf{Y}$ \\
\hline
\end{tabular}

The following observations are based on Table G-3:

- $B o C>0$ for combined retrofit in 10 of 10 scenarios

- $B o C>0$ for attic insulation and wall insulation retrofits in 5 of 5 heating scenarios

- $B o C>0$ for low-e window retrofit in 5 of 5 cooling scenarios

- $B o C>0$ for 6 of 6 retrofits in the $\mathrm{C} 5$ (low consumption) space-heating scenario

- Remaining retrofits and scenarios show mixed $B o C$

- Cases with greater savings (e.g., insulation in heating climate, windows in cooling climate) have greater $B o C$.

$B o C$ calculations for the combined retrofit cases (L300EX) are presented in Table G-4 to provide an estimate of the benefit of calibration. In addition to the decrease in mean absolute error (million Btu or $\mathrm{kWh}$ ), results are also presented in Table G-4 as a percentage of the average reference energy savings prediction ("\% Avg Ref Savings") and as monetary values (\$/yr) assuming $\$ 12.58 /$ million Btu gas cost and $\$ 0.116 / \mathrm{kWh}$ electricity cost (EIA 2009a, 2009b). Cost conversion factors are discussed in Judkoff et al. (2010). 
Table G-4. Benefit of Calibration for Combined Retrofit Cases (L300EX) in Working Group Field Trial

\begin{tabular}{|c|c|c|c|c|c|}
\hline \multicolumn{6}{|c|}{$\begin{array}{l}\text { Decrease in Mean Absolute Error of Energy Savings Predictions } \\
\text { ("BoC," see Equation G3) }\end{array}$} \\
\hline Heating, Colorado Springs & $\mathrm{C} 3 \mathrm{H}$ & $\mathrm{C} 4 \mathrm{H}$ & $\mathrm{C} 5 \mathrm{H}$ & $\mathrm{C} 6 \mathrm{H}$ & $\mathrm{C} 7 \mathrm{H}$ \\
\hline (million Btu) & 3.1 & 16.5 & 18.6 & 8.3 & 6.3 \\
\hline (\% of Avg Ref Savings) & $4.5 \%$ & $18.0 \%$ & $39.9 \%$ & $9.8 \%$ & $10.9 \%$ \\
\hline (\$/yr assuming $\$ 12.58 /$ million Btu) & $\$ 39$ & $\$ 208$ & $\$ 234$ & $\$ 104$ & $\$ 80$ \\
\hline Cooling, Las Vegas & $\mathrm{C} 3 \mathrm{C}$ & $\mathrm{C} 4 \mathrm{C}$ & C5C & $\mathrm{C} 6 \mathrm{C}$ & C7C \\
\hline (kWh) & 356.9 & 395.3 & 521.8 & 294.0 & 457.9 \\
\hline (\% of Avg Ref Savings) & $13.0 \%$ & $10.9 \%$ & $20.8 \%$ & $8.5 \%$ & $17.3 \%$ \\
\hline (\$/yr assuming $\$ 0.116 / \mathrm{kWh})$ & $\$ 41$ & $\$ 46$ & $\$ 61$ & $\$ 34$ & $\$ 53$ \\
\hline
\end{tabular}

As seen in Table G-4, calibration decreased the mean absolute error by 3.1-18.6 million Btu (heating) and $294.0-521.8 \mathrm{kWh}$ (cooling) for the fully random combined retrofit scenarios. In terms of percentage of the average reference program energy savings predictions, the decrease ranged from $4.5-39.9 \%$ (heating) and $8.5-20.8 \%$ (cooling). Translated into U.S. dollars assuming conversion factors for gas and electricity prices noted above, the benefit of calibration ranged from \$39-234 (heating) and \$34-61 (cooling). For the space-heating retrofits, the benefit was largest for the low and high base-case heating consumption scenarios, followed by the mid-low and mid-high scenarios, with the near-nominal scenario showing the least benefit. For the space-cooling retrofits, the benefit of calibration is positive, but does not vary as greatly with the degree of calibration required for a given scenario.

The benefit of calibration for the heating cases is generally greater than the benefit for the cooling cases. One possible explanation is that the retrofit measures for the cooling cases lead to less energy savings in terms of the overall pre-retrofit energy consumption, especially for retrofits that have more impact in heating climates. Thus, even with a fairly substantial $9-21 \%$ BoC (\% of Avg Ref Savings), the economic benefit is less substantial.

\section{G.2.4 Conclusions}

This analysis is one of many approaches that can be used to estimate the benefit of calibration. Results of the analysis are specific to the base case house and input ranges defined in the test, as well as to the randomly generated scenarios, and to the reference and Working Group simulations used for the simulation trial. Additionally, the mean absolute error does not distinguish between underprediction and overprediction. In this context, Figures G-3 through G-12 show how calibration affects the average energy savings predictions and can be examined for trends in overprediction and underprediction. 
Based on this preliminary analysis, energy savings predictions are generally improved by calibration. Improvement is not seen for every retrofit measure and calibration scenario, but calibration tends to improve predictions for:

- Scenarios where there is a large difference between utility bills versus the energy consumption predicted using an uncalibrated model (i.e., where a larger degree of calibration is required)

- Individual retrofit measures with robust energy savings (e.g., insulation in heating climate, windows in cooling climate)

- Combinations of retrofit measures that maximize energy savings.

\section{G.3 Improvements to Tested Software and Importance of Simulation Trials}

As a result of the BESTEST-EX simulation trials, the working group participants documented eight software revisions and two input errors. The proprietary nature of participant programs does not allow disclosure of details. However, the working group participants indicated that the diagnostic logic associated with specific parameter variations of the test cases helped to isolate problems. Additionally, NREL clarified portions of the test specification related to the documented input errors. Therefore, the simulation trials are beneficial in that BESTEST-EX is already driving improvements to retrofit software, and the simulation trials drive improvements to the test procedure. 


\section{Appendix H Definitions}

approximate input: an input for which an approximate input range has been defined; see listing in Section 1.3.1.2. Also see approximate input range.

approximate input range: the specified range of possible values for an approximate input that forms the basis uncertainty range for selecting calibrated approximate inputs for the tested programs (see Section 1.3.1.2), and from which explicit inputs are randomly selected in accordance with the process described in Appendix F. Also see calibrated input and explicit input.

calibrated input or calibrated approximate input: inputs for tested programs that are determined based on specified approximate input ranges and nominal input values using calibration to obtain agreement with base-case reference utility billing data. Also see approximate input range and nominal input.

cavity albedo: see solar lost through window.

combined radiative and convective surface coefficient: constant of proportionality relating the rate of combined convective and radiative heat transfer at a surface to the temperature difference across the air film on that surface.

direct solar radiation: the solar radiation received from the sun without having been scattered by the atmosphere or other objects such as the ground; this is also called beam or direct-beam radiation.

diffuse solar radiation: the solar radiation received from the sun after its direction has been changed by scattering by the atmosphere or other objects such as the ground.

effective coefficient of performance (COP): the ratio of sensible heat extraction from the space by the space cooling equipment divided by the electricity use measured at the meter, including all losses associated with system efficiency, air distribution, etc.; latent load is not considered.

effective heating efficiency: the ratio of heat provided to the space by the furnace divided by the furnace gas use measured at the meter, including all losses associated with furnace efficiency, air distribution, etc.

explicit input: inputs for simulations used to develop reference utility billing data that are randomly selected from within specified approximate input ranges according to the process described in Appendix F. Also see approximate input range.

exterior film: as used in Section 1, see combined radiative and convective surface coefficient.

extinction coefficient: the proportionality constant $\mathrm{K}$ in Bouguer's Law $((d I)=(I \mathrm{~K} d x))$ where $I$ is the local intensity of solar radiation within a medium and $x$ is the distance the radiation travels through the medium.

film coefficient: see combined radiative and convective surface coefficient.

hemispherical infrared emittance: average directional infrared emittance over a hemispherical envelope over the surface. Also see infrared emittance.

incidence angle: angle defined by the intersection of a line normal to a surface and a ray that strikes that surface.

index of refraction: relates the angle of refraction $\left(x_{2}\right)$ to the angle of incidence $\left(x_{1}\right)$ at the surface interface of two media according to Snell's Law $\left(n_{1} \sin \left(x_{1}\right)=n_{2} \sin \left(x_{2}\right)\right)$ where $n_{1}$ and $n_{2}$ are indices of refraction for each medium.

infiltration: the leakage of air through any building element (walls, windows, doors, etc.).

infrared emittance: the ratio of the infrared spectrum radiant flux emitted by a body to that emitted by a blackbody at the same temperature and under the same conditions. 
interior film: as used in Section 1, see combined radiative and convective surface coefficient.

interior solar distribution: the fraction of transmitted solar radiation incident on specific surfaces in a room. Also see solar distribution fraction.

internal gains: heat gains generated inside the space or zone.

latent heat: the change in enthalpy associated with a change in humidity ratio, caused by the addition or removal of moisture.

nominal input: an input value as specified for the building physics base case (Case L200EX-P, see Section 1.2.1).

non-proportional-type thermostat: a thermostat that provides two-position (ON/OFF) control.

raised floor exposed to air: floor system where the air temperature below the floor is assumed to equal the outdoor air temperature, the underside of the conditioned zone floor has an exterior film coefficient consistent with a "rough" surface texture and zero wind speed, and the conditioned zone floor exterior surface (surface facing the raised floor) receives no solar radiation. Also see Section 1.2.1.5.

sensible heat: the change in enthalpy associated with a change in dry-bulb temperature caused by the addition or removal of heat.

shading coefficient (SC): ratio of solar heat gain coefficient (SHGC) for a given window or window system to that for direct normal incident solar radiation for unshaded clear reference glass (SHGC $=$ 0.87). Also see solar heat gain coefficient.

shortwave: refers to the solar spectrum; e.g., in this test procedure the terms solar absorptance and shortwave absorptance are used interchangeably.

solar absorptance: the ratio of the solar spectrum radiant flux absorbed by a body to that incident on it.

solar distribution fraction: the fraction of total solar radiation transmitted through the window(s) that is absorbed by a given surface or retransmitted (lost) back out the window(s).

solar heat gain coefficient (SHGC): a dimensionless ratio of solar heat gains to incident solar radiation, including transmittance plus inward flowing fraction of absorbed solar radiation; for windows, SHGC is dependent on solar incidence angle.

solar lost: see solar lost through window.

solar lost through window: the fraction of total solar radiation transmitted through the window(s) that is reflected by opaque surfaces and retransmitted back out the window(s).

zone air temperature: the temperature of just the zone air, not including infrared radiation from the interior surfaces; such a temperature would be measured by a sensor housed in a well-aspirated containment shielded by a material with a solar and infrared reflectance of one; well-mixed air is assumed. 


\section{Appendix I Recommendations for Future Work}

The BESTEST-EX Working Group (2009-2010) has given NREL many insights into the trends observed in the field for retrofit efforts in both the weatherization and private sector contexts. One important observation is the tendency for even the most advanced simulation programs to over-predict the energy use in pre-retrofit older poorly insulated homes, and to also over-predict savings from retrofits. Practitioners in the low income weatherization program, and related utility bill data, indicate that it is not uncommon for the prediction of energy usage and savings to be roughly double the average of actual measurements (Berry and Gettings 1998; Blasnik 2010; Dalhoff 1997; Pigg 2001; Sharp 1994). It is crucial, as the nation embarks on a large scale retrofit effort, to identify and correct the sources of these errors. To solve this question, a serious effort is needed to validate building energy simulation programs against high quality measured data. Empirical experiments are needed that range from gathering and analyzing detailed data (to address resolving building physics modeling related errors), to analyzing large statistical samples (to understand the average impact of occupant behavior on energy savings).

Comparisons with empirical data will drive improvements to the state-of-the-art reference programs used in software-to-software comparative tests. Software-to-software comparative tests complement comparisons with empirical data because they provide robust capability for diagnosing software errors by directly analyzing sensitivity to input variations. (Judkoff and Neymark 2006) Software-to-software comparative tests are also useful for identifying simulation input specification requirements needed for developing useful comparisons with high-quality measured data (Judkoff et al. 2008; Neymark et al. 2005). Therefore, in parallel with validating building energy simulation programs versus measured data, refinements to and expansion of the BESTEST-EX Phase 1 comparative test cases could address areas of building physics modeling and model calibration not covered in BESTEST-EX Phase 1.

Recommendations for future work related to BESTEST-EX, discussed below, are divided into the general categories of:

- Empirical data checks; see Section I.1.

- Additional test cases; see Section I.2.

- Revisions to existing test cases; see Section I.3.

\section{I.1 Empirical Data Checks}

Initial work on empirical data checking should address the following preliminary questions related to using empirical data to quantify the accuracy of simulation tools:

- What defines good quality empirical data?

- Satisfactory documentation of billing data and other measurements, and their related uncertainties, for accurate comparison with simulation predictions?

- Is sub-metered data available?

- Satisfactory documentation of the building, for sufficiently accurate characterization of simulation inputs?

○ Satisfactory documentation of occupant behavior?

○ Other?

- How accessible are various existing data sets?

- How much existing data is available where specific buildings were sufficiently documented and monitored pre- and post-retrofit?

- Are pre-retrofit audits and post-retrofit testing documentation or audits available? 
- Should new data be gathered in existing homes?

- Should custom empirical validation experimental facilities be constructed that allow:

- Empirical determination of simulation inputs?

- Side-by-side parametric sensitivity tests?

○ Reconfigurable test buildings and instrumentation?

After addressing comparison of simulation tools with empirical data, the following questions related to improving BESTEST-EX to better match empirical data may be addressed:

- Are there input specifications in BESTEST-EX that need further update based on comparisons with reference simulations and existing empirical data?

- How can BESTEST-EX and its reference simulation results be compared with existing empirical data for a given home?

○ Normalize existing data for floor area, climate, and other characteristics?

- Is it possible to define a separate process for developing a test suite where simulation predictions are directly compared with empirical data?

\section{I.2 Additional Test Cases}

Additional test cases being considered for inclusion in future versions of BESTEST-EX are categorized by mechanical equipment and building thermal fabric (enclosure) tests as listed in Sections I.2.1 and I.2.2, respectively. Where possible, existing BESTEST/ASHRAE Standard 140 (ANSI/ASHRAE 2007; Judkoff and Neymark 1995a, 1995b; Neymark and Judkoff 2002, 2004; Purdy and Beausoleil-Morrison 2003) and RESNET (2007) test suites may be considered for direct use, or as the basis for additional cases.

\section{I.2.1 Mechanical Equipment Tests}

- $\quad$ Space heating equipment

- System replacement (to higher efficiency)

○ Fuel switching (e.g. air- and/or ground-source heat pumps versus gas-fired furnace or hydronic systems)

○ Secondary items: pilot lights, humidifiers, etc.

- Space cooling equipment

- Humid climate system replacement

○ Dry climate system replacement

- Duct sealing and insulation

- Domestic hot water

○ Tank and pipe insulation

- System replacement (to higher efficiency)

○ Supply temperature modeling

- Ventilation

○ Fans

$\circ$ Air-to-air heat exchanger 


\section{I.2.2 Building Thermal Fabric (Enclosure) Tests}

- Internal window shading

- Other floor constructions and related retrofits

- Vented crawl space/closed, conditioned crawl space

- HERS BESTEST (Judkoff and Neymark 1995a) slab-on-grade and basement cases

○ IEA 34/43 slab-on-grade tests (Neymark et al. 2008), selected "b"-series and "c"-series cases

- Lighting and household appliances (not including space conditioning and DHW equipment)

- Thermal mass

- Hot/humid climate air-seal retrofit.

\section{I.3 Revisions to Existing Test Cases}

During development of the initial version of BESTEST-EX, a number of possible revisions were identified. Possible revisions are categorized by building physics tests and calibration tests as listed in Sections I.3.1 and I.3.2, respectively. Changes to building physics tests also affect the calibration tests.

\section{I.3.1 Possible Revisions to Building Physics Tests}

- Review the outcome of empirical data checks (see above Section I.1) to determine if changes are needed to the base building or retrofit specifications to better match empirical evidence.

- Apply separate types of window retrofits for heating and cooling climates.

- Revise the default combined interior surface coefficients (for programs that do not automatically calculate surface heat transfer); see Appendix C, Section C.1.1.1.

- Consider refinements to default combined exterior surface coefficients (for programs that do not automatically calculate surface heat transfer).

- Infiltration:

- Investigate possible causes of the difference between air-sealing retrofit energy savings calculated by the reference simulations versus estimates derived from utility billing data; see Appendix D, Section D.4.

- Consider addressing infiltration heat recovery.

- More detailed examination of ASHRAE (2005) stack and wind coefficients.

- Investigate varying terrain types and shelter classes.

- Apply SUNREL's weather-driven infiltration model to generate its results.

○ Consider reducing effective leakage area in Case L210EX-P by $100 \mathrm{in}^{2}$ rather than by $98.1 \mathrm{in}^{2}$ to match L210EX-C, for better benefit of calibration comparison.

- Apply non-constant ground reflectance to account for the presence of winter snow.

- Revise the EnergyPlus reference simulations to include more precise window height input; preliminary sensitivity tests indicate this would lead to about a $2 \%$ increase in the interior combined heat transfer coefficient for single-pane window.

- Add internal mass for furnishings. 
- Include ANSI/ASHRAE Standard 140 (2007) modeler report templates, and consider an additional report template to document calibration methods without disclosing proprietary information.

\section{I.3.2 Possible Revisions to Calibration Tests}

- Consider developing a version of the procedure for testing programs with automated calibration; such a version would have calibrated savings results available so that results would not have to be reviewed by a third party.

- Apply an 8760-hour varying internal gains schedule, in place of the current 24-hour varying day schedule repeated for all days; this adds more realistic difficulty to base-case utility bill calibration for the tested programs.

- Include input uncertainty (approximate input ranges) for window U-value and SHGC.

- Consider revising the current approximate input ranges; see Appendix F, Table F-1.

- Include external shading in the space cooling cases.

- Fix the exterior wall and gable solar absorptance at 0.6 ; only apply the current solar absorptance approximate input range to the roof. 


\section{REPORT DOCUMENTATION PAGE}

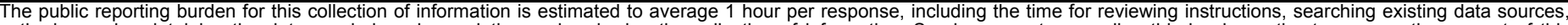

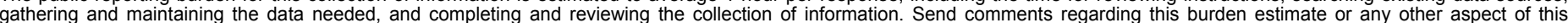

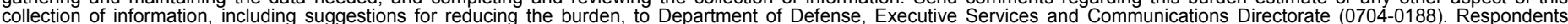

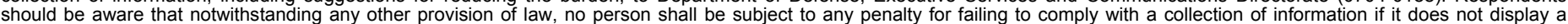

should be aware that notwithstand

PLEASE DO NOT RETURN YOUR FORM TO THE ABOVE ORGANIZATION.

\section{REPORT DATE (DD-MM-YYYY) August 2010}

4. TITLE AND SUBTITLE

Building Energy Simulation Test for Existing Homes (BESTEST-EX)

Phase 1 Test Procedure: Building Thermal Fabric Cases

\section{DATES COVERED (From - To)}

5a. CONTRACT NUMBER

DE-AC36-08-GO28308

5b. GRANT NUMBER

5c. PROGRAM ELEMENT NUMBER

5d. PROJECT NUMBER

NREL/TP-550-47427

5e. TASK NUMBER

ARRB.1000

5f. WORK UNIT NUMBER
7. PERFORMING ORGANIZATION NAME(S) AND ADDRESS(ES)

National Renewable Energy Laboratory

1617 Cole Blvd.

Golden, CO 80401-3393

\section{PERFORMING ORGANIZATION REPORT NUMBER \\ NREL/TP-550-47427}

9. SPONSORING/MONITORING AGENCY NAME(S) AND ADDRESS(ES)

10. SPONSOR/MONITOR'S ACRONYM(S) NREL

11. SPONSORING/MONITORING AGENCY REPORT NUMBER

12. DISTRIBUTION AVAILABILITY STATEMENT

National Technical Information Service

U.S. Department of Commerce

5285 Port Royal Road

Springfield, VA 22161

13. SUPPLEMENTARY NOTES

14. ABSTRACT (Maximum 200 Words)

The U.S. Department of Energy tasked NREL to develop a process for testing the reliability of models that predict retrofit energy savings, including their associated calibration methods. DOE asked NREL to conduct the work in phases so that a test procedure would be ready should DOE need it to meet legislative requirements related to residential retrofits in FY 2010. This report documents the initial "Phase 1" test procedure.

\section{SUBJECT TERMS}

bestest; retrofit energy savings; building energy simulation

\begin{tabular}{|c|c|c|}
\hline \multicolumn{3}{|c|}{ 16. SECURITY CLASSIFICATION OF: } \\
\hline $\begin{array}{l}\text { a. REPORT } \\
\text { Unclassified }\end{array}$ & $\begin{array}{l}\text { b. ABSTRACT } \\
\text { Unclassified }\end{array}$ & $\begin{array}{l}\text { c. THIS PAGE } \\
\text { Unclassified }\end{array}$ \\
\hline
\end{tabular}

\begin{tabular}{l|l|} 
17. LIMITATION & 18. \\
OF ABSTRACT & OF PAGES \\
UL &
\end{tabular}

19a. NAME OF RESPONSIBLE PERSON

19b. TELEPHONE NUMBER (Include area code) 